Universidade de São Paulo

Faculdade de Filosofia, Ciências e Letras de Ribeirão Preto

Alexandre Henrique de Martini

Analiticidade da função exponencial generalizada para argumentos complexos e suas implicações 

Alexandre Henrique de Martini

\section{Analiticidade da função exponencial generalizada para argumentos complexos e suas implicações}

Tese apresentada à Faculdade de Filosofia, Ciências e Letras de Ribeirão Preto da Universidade de São Paulo como parte das exigências para a obtenção do título de Doutor em Ciências.

Área de Concentração:

Física Aplicada à Medicina e Biologia.

Orientador:

Alexandre Souto Martinez.

Versão corrigida

Versão original disponível na FFCLRP-USP

Ribeirão Preto 
Autorizo a reprodução e divulgação total ou parcial deste trabalho, por qualquer meio convencional ou eletrônico, para fins de estudo e pesquisa, desde que citada a fonte.

\section{FICHA CATALOGRÁFICA}

Martini, Alexandre Henrique de

Analiticidade da função exponencial generalizada para argumentos complexos e suas implicações / Alexandre Henrique de Martini; orientador: Alexandre Souto Martinez. - - Ribeirão Preto, 2020.

77 f. : il.

Tese (Doutorado) - - Faculdade de Filosofia, Ciências e Letras de Ribeirão Preto, Universidade de São Paulo, 2020.

Inclui Bibliografia.

1. Transformada de Fourier generalizada 2. Log-periodicidade 3. Funções generalizadas e propriedades 
Nome: Martini, Alexandre Henrique de

Título: Analiticidade da função exponencial generalizada para argumentos complexos e suas implicações

Tese apresentada à Faculdade de Filosofia, Ciências e Letras de Ribeirão Preto da Universidade de São Paulo como parte das exigências para a obtenção do título de Doutor em Ciências.

Aprovado em:

\section{Banca Examinadora}

$\operatorname{Prof}(\mathrm{a}) \cdot \operatorname{Dr}(\mathrm{a})$.

Julgamento:

$\operatorname{Prof}(\mathrm{a}) \cdot \operatorname{Dr}(\mathrm{a})$.

Julgamento:

$\operatorname{Prof}(\mathrm{a}) \cdot \operatorname{Dr}(\mathrm{a})$.

Julgamento:

$\operatorname{Prof}(\mathrm{a}) \cdot \operatorname{Dr}(\mathrm{a})$.

Julgamento:

$\operatorname{Prof}(\mathrm{a}) \cdot \operatorname{Dr}(\mathrm{a})$.

Julgamento:
Instituição:

Assinatura:

Instituição:

Assinatura:

Instituição:

Assinatura:

Instituição:

Assinatura:

Instituição:

Assinatura: 
À minha esposa Flávia, pelo incentivo, parceria e cumplicidade. Aos filhos, Andrew, Ashley, Anry e Antonella, meus motivadores. 


\section{Agradecimentos}

Meus sinceros agradecimentos ao meu orientador, o Prof. Dr. Alexandre Souto Martinez. Por toda paciência, dedicação, cobrança e indicações precisas. Sempre me senti muito bem amparado, tanto em vias acadêmicas, quanto em momentos de aconselhamento ou de descontração, tudo a seu tempo.

Aos colegas de laboratórios, que, cada um a seu modo, colaboraram para o meu crescimento. Ao amigo, Gilberto Nakamura, que dedicou tempo e paciência, seja para me apresentar ao mundo Linux, ou em dar importantes referências para meus estudos. Muito obrigado! Ao Guilherme Contesini, que sempre foi boa companhia para o Restaurante Universitário, para dicas de leitura e algumas ideias, tanto para pesquisa quanto para a vida. Ao amigo Fernando Meloni, sempre com um bom papo, um ótimo café, muitas ideias e várias risadas. Ao Cristiano Granzotti, pelas incontáveis guloseimas deixadas para que a "ciência pudesse fluir", além da oportunidade de conhecer um interessante campo de estudos através de seus seminários muito bem apresentados. Ao José Renato Alcarás, por todo zelo com a ciência e com escrita científica e principalmente pelas importantes observações feitas neste trabalho. Ao Olavo Menin por ter aberto as portas deste laboratório para mim. A todos os outros que passaram pelo Laboratório de Modelagem de Sistemas Complexos LMSC - e, de uma forma ou outra, colaboraram com minha formação. Muito obrigado a todos.

Aos docentes do departamento de Física e do departamento de Computação e Matemática. Por toda dedicação, excelentes aulas e troca de experiências. Aos servidores, secretárias e demais funcionários, por todos os serviços prestados, por todas as informações, por toda dedicação. Sem vocês minha formação não seria completa. Muito obrigado.

Ao MEC e ao Instituto Federal de Educação Ciência e Tecnologia - IFSP, por todo apoio, principalmente no que diz respeito ao afastamento. Graças a esta oportunidade foi possível realizar um trabalho de excelência. Aos colegas do campus Sertãozinho, de todos os setores e de modo especial à DAGP, que através de seus representantes, sempre foram solícitos em minhas dúvidas (que não foram poucas). Aos colegas da matemática: obrigado por se desdobrarem em aulas para que eu pudesse terminar essa capacitação. Espero poder, de alguma forma e em algum momento, retribuir todo esforço. Muito obrigado.

A toda minha família. Desde de meus avós, Gilda e Antônio, sempre ávidos por minha formação, passando por meus pais, meus irmãos, minha esposa Flávia (que em muitos momentos fez por nós dois) até chegar nos meus 4 filhos. Cada um colaborou, a seu modo, um pouquinho para que eu chegasse aqui. Muito obrigado. 
Eu sou um matemático; a matemática preenche a minha vida... - Laurent Schwartz 


\section{Resumo}

MARTINI, A. H. Analiticidade da função exponencial generalizada para argumentos complexos e suas implicações. 2020. 77 f. Tese (Doutorado - Programa de Pós-Graduação em Física Aplicada à Medicina e Biologia) - Faculdade de Filosofia, Ciências e Letras de Ribeirão Preto, Universidade de São Paulo, Ribeirão Preto, 2020.

Funções generalizadas ganharam amplo espaço de discussão e divulgação a partir da década de 1990, no contexto da entropia não extensiva de Tsallis. As funções logaritmo e exponencial generalizadas são a base para muitos estudos e aplicações: a função logaritmo generalizado é a transformação de Box-Cox enquanto a função exponencial generalizada é solução da equação diferencial de cinética química de ordem arbitrária. Apresentamos, nesta tese, uma extensão para a função exponencial generalizada com argumentos complexos, $\mathrm{e}_{\lambda}(z)$, que, a exemplo da função convencional, é analítica em todo plano complexo. Uma generalização consistente desta função abre espaço para outras generalizações relacionadas a ela. A nossa proposta de generalização da função exponencial leva a expressão e ${ }^{i \omega t}$ em $\left[\mathrm{e}_{\lambda}(t)\right]^{i \omega}$, o que traz vantagens na generalização da transformada de Fourier, de funções trigonométricas, hiperbólicas e log-periódicas. Nelas são preservadas importantes características e propriedades, além de extrapolar a aplicabilidade em vários contextos, tais como, fraturas de materiais, mercado financeiro, previsão de terremotos, entre outros. A nova generalização da função exponencial proporcionou um ganho significativo nas demais generalizações e, desta forma, pode-se dizer que esta generalização pertence ao seleto grupo das generalizações convenientes.

Palavras-chave: 1. Transformada de Fourier generalizada 2. Log-periodicidade 3. Funções generalizadas e propriedades 


\section{Abstract}

MARTINI, A. H. Analyticality of the generalized exponential function for complex arguments and its implications. 2020. $77 \mathrm{f}$. Thesis (Ph.D. - Postgraduate program in Physics Applied to Medicine and Biology) - Faculty of Philosophy, Sciences and Letters, University of São Paulo, Ribeirão Preto, 2020.

Generalized functions gained ample space for discussion and dissemination since the 1990s, in the context of Tsallis' non-extensive entropy. The generalized logarithm and exponential functions are the basis for many studies and applications: the generalized logarithm function is the Box-Cox transformation while the generalized exponential function is a solution to the differential equation of chemical kinetics of arbitrary order. In this thesis, we present a new generalization for the generalized exponential function with complex arguments $\mathrm{e}_{\lambda}(z)$, which, like the conventional function, is analytical in the whole complex plane. A consistent generalization of this function makes room for other generalizations related to it. Our proposal to generalize the exponential function takes the expression $\mathrm{e}^{i \omega t}$ to $\left[\mathrm{e}_{\lambda}(t)\right]^{i \omega}$, which has advantages in generalizing the Fourier transform, of trigonometric, hyperbolic and log-periodic functions. In this generalization, important characteristics and properties are preserved, in addition to maintaining applicability in various contexts, such as material fractures, financial market, earthquake prediction, among others. The new generalization of the exponential function provided a significant gain in the other generalizations and, thus, it can be said that this generalization belongs to the select group of convenient generalizations.

Key-words: 1. Generalized Fourier transform 2. Log-periodicity 3. Generalized functions and properties 


\section{Lista de Figuras}

2.1 (a)Família de funções $f_{\lambda}(t)$ para valores de $\lambda$ iguais a 0,1/2,2 e 3 . Note que $f_{\lambda}(t)$ tem comportamento hiperbólico para $\lambda<1$ e de lei de potência para $\lambda>1$. (b)Família de funções $f_{\lambda}^{-1}(t)$ para valores de $\lambda$ iguais a $0,1 / 2,2$, e 3 . Para $\lambda=2$ temos a função identidade $f_{2}^{-1}(t)=t$. Para $\lambda<2$ o comportamento é hiperbólico. Já para $\lambda>2$ temos leis de potência. . . . . . . . . . . . . . . 7

2.2 Função logaritmo generalizado $\ln _{\lambda}(t)$ para os valores de $\lambda$ iguais a $-1,0,1$, e 3 . Curvas mantém importantes características da função $\ln (t)$ tradicional, tais como, ser estritamente negativa para os valores de $t<1$, nula em $t=1$ e positivas para $t>1$. É possível observar ainda uma simetria de inversão $\lambda_{N}=2-\lambda . \ldots . \ldots . \ldots 9$

2.3 (a)Função logaritmo generalizado com parâmetro par: $\lambda=2 n, n \rightarrow$ $\infty$. Neste caso a função se configura como degrau com $\ln _{\infty}(t)=0$ no intervalo $-1 \leq t \leq 1$ e $\ln _{\infty}(t)=\infty$ fora dele.(b) Função logaritmo generalizado com parâmetro ímpar: $\lambda=2 n-1, n \rightarrow \infty$. Neste caso a função também é Heaviside com praticamente as mesmas características do caso anterior, porém no intervalo $t<1, \ln _{\infty}(t)=-\infty$. . . 10

2.4 Função exponencial generalizada $e_{\lambda}(t)$ e seus comportamentos para valores de $\lambda=-3$ : função hiper exponencial com divergência em $t=$ $1 / 3 ; \lambda=0$ : exponencial convencional; $\lambda=2$ : função raiz quadrada; $\lambda=3$ : raiz cúbica.

2.5 Função exponencial generalizada $e_{\lambda}(t)$ para valores "grandes" de $\lambda$. É possível observar que conforme $\lambda$ aumenta a curva tende a uma reta em $y=1$. Neste exemplo $\lambda=500, \lambda=1000, \lambda=5000$ e $\lambda=10000 . \quad 20$ 
2.6 Possibilidades de interpretação da função e ${ }^{i \omega t}$ após a generalização. Note que $\mathrm{e}^{i \omega t}=\left(\mathrm{e}^{\omega t}\right)^{i}=\left(\mathrm{e}^{t}\right)^{i \omega}$. Contudo, $\mathrm{e}_{\lambda}(i \omega t) \neq\left[\mathrm{e}_{\lambda}(\omega t)\right]^{i} \neq$ $\left[\mathrm{e}_{\lambda}(t)\right]^{i \omega}$. Nota-se que, $\mathrm{e}_{\lambda}(i \omega t)$ não é uma expressão analítica. Por outro lado $\left[\mathrm{e}_{\lambda}(\omega t)\right]^{i}$ não favorece generalização de outras estruturas, como por exemplo, a transformada de Fourier. . . . . . . . . . . . . . 24

2.7 Comportamento de algumas curvas da função $\mathrm{e}_{\lambda}(t)$ com saída em $\mathbb{C}$. a) Parte real da função $\mathrm{e}_{\lambda}(t)$ para os valores de $\lambda=-2, \lambda=0, \lambda=$ $2, \lambda=3$. b) Parte imaginária da função $\mathrm{e}_{\lambda}(t)$ para os valores de $\lambda=$ $-3, \lambda=-2, \lambda=2, \lambda=3$. Pode-se perceber que, nestes exemplos, a função tem como imagem números reais ou números complexos do tipo $z=a+b i$. Nunca imaginários puros.

4.1 Exemplo de mapeamento com $w=f(z)=z^{2}$. Se $z$ é tal que $z=x+$ $i y$, então $f(z)=u(x, y)+i v(x, y) \operatorname{com} u(x, y)=x^{2}-y^{2}$ e $v(x, y)=2 x y .43$

4.2 Diferentes possibilidades para a generalização de $f(t)=e^{i \omega t}: \mathrm{e}_{\lambda}(i \omega t)$, $\left[\mathrm{e}_{\lambda}(\omega t)\right]^{i}$ e finalmente $\left[\mathrm{e}_{\lambda}(t)\right]^{i \omega}$. Exemplo para cada uma das curvas com $t \geq 0, \lambda=1$ e $\omega=20$. Curvas oscilam, com frequências bem diferentes, para $\left[\mathrm{e}_{\lambda}(\omega t)\right]^{i}$ e $\left[\mathrm{e}_{\lambda}(t)\right]^{i \omega}$. Para $\mathrm{e}_{\lambda}(i \omega t)$ tem-se uma função constante. . . . . . . . . . . . . . . . . 45

4.3 O logaritmo natural mapeia um segmento de linha reta em dois segmentos de linha paralelos com direções opostas no plano complexo. (a) O primeiro segmento de linha (tracejado) varia de $-\infty$ a $t_{c}$, enquanto o segundo segmento de linha começa em $t_{c}$ e continua até $\infty$. (b) O mapa conforme estende o primeiro segmento de linha em a) e o desloca por $i \pi / \lambda$ (tracejado).

5.1 Exemplos de funções log-periódicas: $f(x)=\cos [5 \ln (x)]$ inicia oscilando rapidamente e aos poucos muda para uma oscilação mais lenta; $g(x)=\cos [5 \ln (10-x)]$ inicia oscilando lentamente e, à medida que se aproxima de 10, tem oscilação acelerada. Aqui, o valor 10 é o ponto de acumulação. . . . . . . . . . . . . . . . . . . . . 66 66 
5.2 (a) Função cosseno generalizado com valores de $\lambda=0, \lambda=1, \lambda=3$ e $\lambda=-3$. (b) Função seno generalizado com valores de $\lambda=0$, $\lambda=1, \lambda=3$ e $\lambda=-3$. Em ambos exemplos foram obtidas funções oscilatórias e limitadas, apenas para $\lambda=0$. As demais curvas não são limitadas e não oscilam. . . . . . . . . . . . . . . . . . . . . 68

5.3 (a) Nova generalização para a função cosseno com valores de $\lambda=1$, $\lambda=2, \lambda=3$ e $\omega=5$. (b) Nova generalização para a função seno com valores de $\lambda=1, \lambda=2, \lambda=3$ e $\omega=5$. Em ambos exemplos foram obtidas funções oscilatórias e limitadas, característica peculiar das funções trigonométricas. Além disso, é observável, nas duas funções, pontos de acumulação em $t=-1 / \lambda . \ldots . . . \ldots 73$

5.4 Partes imaginárias das funções $\mathrm{c}_{2}(3, t)$ e $\mathrm{s}_{2}(3, t)$ - o conjunto imagem das duas funções é o mesmo. Observa-se ainda ponto de acumulação próximo da origem (mais precisamente em $t=-1 / 3$ ) . . . . . 74

5.5 Parte imaginária da função $c_{\lambda}(5, t)$ para $\lambda=2.0, \lambda=2.25$ e $\lambda=2.5$. Função se mantém limitada e oscilatória para cada valor $\lambda$. Observase ponto de acumulação próximo à origem. . . . . . . . . . . . . . . 75

5.6 (a) Partes real e imaginária da função $c_{\lambda}(3, t)$ para $\lambda \rightarrow \infty$. A Parte real claramente tende a 1 enquanto a imaginária a 0. (b) Partes real e imaginária da função $\mathrm{s}_{\lambda}(3, t)$ para $\lambda \rightarrow \infty$. Tanto parte real quanto imaginária tendem a $0 \ldots \ldots \ldots 76$

5.7 Parte real da função $\mathrm{s}_{3}(\omega, t)$ para $\omega=3, \omega=4$ e $\omega=5$. Função se mantém limitada e oscilatória, aumentando o intervalo do conjunto imagem conforme o aumento do valor da frequência $\omega . \quad$. . . . . . . 77

5.8 (a) Nova generalização para a função cosseno hiperbólico com valores de $\lambda=1, \lambda=2$ e $\lambda=3$, além de $\omega=2$. (b) Nova generalização para a função seno hiperbólico com valores de $\lambda=1, \lambda=2$ e $\lambda=3$, além de $\omega=2$. Em ambos exemplos foram obtidas funções semelhantes a ramos de hipérboles. . . . . . . . . . . . . . . . . . . . . 87 
5.9 (a) Parte real da função $\operatorname{ch}_{\lambda}(\omega, t)$ para os valores de $\lambda=1, \lambda=2$ e $\lambda=3$, além de $\omega=2$. (b) Parte real da função $\operatorname{sh}_{\lambda}(\omega, t)$ para os valores de $\lambda=1, \lambda=2$ e $\lambda=3$, além de $\omega=2$. Em ambos exemplos foram obtidas hipérboles completas. . . . . . . . . . . . . . 88

5.10 (a) Função $\operatorname{ch}_{1}(\omega, t)$ para os valores de $\omega=2, \omega=4$ e $\omega=6$. (b) Função $\operatorname{sh}_{1}(\omega, t)$ para os valores de $\omega=2, \omega=4$ e $\omega=6$. Em ambos exemplos mantém-se fixo $\lambda=1$. . . . . . . . . . . . . . . 89

2.1 (a) Função tangente generalizada $t_{\lambda}(5, t)$ para $\lambda=1, \lambda=2$ e $\lambda=3$. Curvas possuem características semelhantes à tangente original. (b) Função cotangente generalizada $\cot _{\lambda}(5, t)$ para valores de $\lambda=1, \lambda=2$ e $\lambda=3$. As curvas se assemelham às obtidas pela função original. . . 107

2.2 Função secante generalizada $\mathrm{s}_{\lambda}(5, t)$ para valores de $\lambda=1, \lambda=2 \mathrm{e}$ $\lambda=3$. Os períodos vão aumentando à medida que $\lambda$ aumenta. . . . . 108

2.3 Função cossecante generalizada $\csc _{\lambda}(5, t)$, para valores de $\lambda=1, \lambda=$ 2 e $\lambda=3 \ldots \ldots \ldots \ldots \ldots$

2.4 Função tangente hiperbólica generalizada $\mathrm{th}_{\lambda}(3, t)$, para valores de $\lambda=-1, \lambda=2$ e $\lambda=3 \ldots \ldots \ldots$. . . . . . . . . . . . . . . . . . . . .

2.5 Função cotangente hiperbólica generalizada $\operatorname{cth}_{\lambda}(3, t)$, para valores de $\lambda=-1, \lambda=2$ e $\lambda=3 \ldots \ldots \ldots 111 \ldots \ldots \ldots \ldots$

2.6 Função secante hiperbólica generalizada $\operatorname{sch}_{\lambda}(3, t)$, para valores de $\lambda=-1, \lambda=2$ e $\lambda=3 \ldots \ldots \ldots \ldots$. . . . . . . . . . . . . . . . . .

2.7 Função cossecante hiperbólica generalizada $\operatorname{csch}_{\lambda}(3, t)$, para valores de $\lambda=-1, \lambda=2$ e $\lambda=3$. 


\section{Sumário}

$\begin{array}{ll}\text { Lista de Figuras } & \text { XV }\end{array}$

1 Introdução 1

2 Funções e Operadores Generalizados 5

2.1 Caminhos para generalização . . . . . . . . . . . . . . . . 6

2.2 Função logaritmo generalizado . . . . . . . . . . . . . . . . . . . . 8

2.3 Operações ou operadores binários . . . . . . . . . . . . . . . . . . . 12

2.4 Operadores generalizados . . . . . . . . . . . . . . . . . 14

2.5 A função exponencial generalizada . . . . . . . . . . . . . . . . . . . . 18

2.6 A exponencial generalizada no plano complexo . . . . . . . . . . . . 24

3 Transformações de Box-Cox $\quad 29$

3.1 Contexto histórico . . . . . . . . . . . . . . . . . . . . . . . . 29

3.2 Transformações do tipo Box-Cox . . . . . . . . . . . . . . . . 31

3.3 Estimativa do parâmetro $\lambda \ldots \ldots . \ldots . \ldots . \ldots . \ldots$

4 Uma Generalização para a Transformada de Fourier $\quad 37$

4.1 A transformada de Fourier . . . . . . . . . . . . . . . . 38

4.2 Generalizando a transformada de Fourier . . . . . . . . . . . . . . . . 40

4.3 Mapeamento e mapeamento conforme . . . . . . . . . . . . . . . . . . 42

4.4 Uma nova extensão da $\lambda$-Transformada de Fourier . . . . . . . . . . . 44

4.5 Distribuição $\lambda$-Delta . . . . . . . . . . . . . . . . . . . . . 48

4.6 Translações generalizadas e outras propriedades . . . . . . . . . . 50

4.6.1 A $\lambda$-TF de um sinal log-periódico . . . . . . . . . . . . . 51

4.6 .2 Conjugação . . . . . . . . . . . . . . . . . . 52

$4.6 .3 \quad \lambda$-TF da derivada . . . . . . . . . . . . . . 53 
4.6.4 Transformada da Integral . . . . . . . . . . . . . . 54

4.6.5 Modulação . . . . . . . . . . . . . . . . 55

4.7 Inverso . . . . . . . . . . . . . . . . . . . . . . . . 55

4.8 Conclusão . . . . . . . . . . . . . . . . . . . . 56

5 Funções Log-periódicas Generalizadas $\quad 59$

5.1 Funções periódicas . . . . . . . . . . . . . . . . . . . . . . . 59

5.1 .1 Funções trigonométricas . . . . . . . . . . . . . . . . 60

5.1 .2 Funções hiperbólicas . . . . . . . . . . . . . . . . 63

5.2 Funções log-periódicas . . . . . . . . . . . . . . . . . . . . 65

5.2.1 Funções trigonométricas e hiperbólicas generalizadas . . . . . 67

5.2 .2 Novas generalizações . . . . . . . . . . . . . . 70

$5.2 .2 .1 \quad$ Paridade. . . . . . . . . . . . . 75

5.2.2.2 Relação Fundamental da Trigonometria . . . . . . . 77

5.2.2.3 Relação de Euler . . . . . . . . . . . . . . . . . . . 78

5.2 .2 .4 Fórmula de De Moivre . . . . . . . . . . . . . 78

5.2.2.5 Soma de $\operatorname{arcos} \ldots \ldots . \ldots . \ldots 79$

5.2.2.6 Produtos .................... 80

5.2.2.7 Derivadas . . . . . . . . . . . . . . . 81

5.2.2.8 Limite fundamental . . . . . . . . . . . . . . . . . . 82

5.2 .2 .9 Integração . . . . . . . . . . . . . . . . . . 83

5.2.3 Integrais de produtos para série de Fourier generalizada . . . . 83

5.2 .4 As funções hiperbólicas generalizadas . . . . . . . . . . . . . 85

5.2 .4 .1 Relações iniciais . . . . . . . . . . . . . 86

5.2 .4 .2 Relação fundamental . . . . . . . . . . . . . . . 90

5.2.4.3 Produtos ....................... 91

5.2.4.4 Derivadas .................... 91

5.2 .4 .5 Integração . . . . . . . . . . . . . . . . . . . 92

5.2.5 Relacionando as funções seno e cosseno generalizadas com as funções hiperbólicas generalizadas . . . . . . . . . . . . . 93

5.2 .6 Exemplo e conclusões . . . . . . . . . . . . . . . . . . 95

6 Conclusão e Perspectivas $\quad 97$ 
Apêndice 2 - Generalização das demais funções Trigonométricas e Hiperbólicas

2.1 Funções trigonométricas generalizadas . . . . . . . . . . . . . . 105

2.1.1 Função tangente generalizada: $t_{\lambda}(\omega, t) \ldots 106$

2.1.2 Função cotangente generalizada: $\cot _{\lambda}(\omega, t) \ldots 106$

2.1.3 Função secante generalizada: $\operatorname{sc}_{\lambda}(\omega, t) \ldots$. . . . . . . . . 108

2.1.4 Função cossecante generalizada: $\csc _{\lambda}(\omega, t) \ldots 108$

2.2 Funções hiperbólicas generalizadas . . . . . . . . . . . . . . 109

2.2.1 Função tangente hiperbólica generalizada: $\operatorname{th}_{\lambda}(\omega, t)$. . . . . 110

2.2.2 Função cotangente hiperbólica generalizada: $\operatorname{cth}_{\lambda}(\omega, t) \quad$. . . 111

2.2.3 Função secante hiperbólica generalizada: $\operatorname{sch}_{\lambda}(\omega, t)$. . . . . 111

2.2.4 Função cossecante hiperbólica generalizada: $\operatorname{csch}_{\lambda}(\omega, t)$. . . 112

Apêndice 3 - Algumas demontrações $\quad 115$

3.1 Relação fundamental da trigonometria . . . . . . . . . . . . . . . 115

3.2 Relação de Euler . . . . . . . . . . . . . . . . . . . . 116

3.3 Produtos entre funções e somas de arcos . . . . . . . . . . . . 116

3.3.1 Produtos ......................... 116

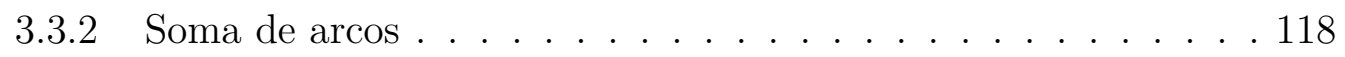

3.4 Limites fundamentais . . . . . . . . . . . . . . . . . . . 122

3.5 Relação fundamental das funções hiperbólicas . . . . . . . . . . . . 123

3.6 Produtos de funções hiperbólicas generalizadas . . . . . . . . . . . . 123

3.7 Relação entre funções trigonométricas e hiperbólicas . . . . . . . . . . 125

$\begin{array}{lr}\text { Referências } & 127\end{array}$ 


\section{Introdução}

Pouco conhecimento faz com que as pessoas se sintam orgulhosas. Muito conhecimento, com que se sintam humildes.

- Leonardo da Vinci

Generalização é a operação intelectual que consiste em reunir numa classe, termo ou proposição, um conjunto de seres ou fenômenos similares. Na ciência, generalizar tem o sentido de agrupar segundo um conjunto de características e propriedades. Idealmente, uma generalização deve considerar apenas critérios objetivos, contudo, generalizações também podem apresentar algum nível de subjetividade. Assim, surge a seguinte dúvida: quando uma generalização é consistente? Ou ainda, quais critérios podem ser utilizados para definir o que é uma boa generalização?

Um dos pioneiros na teoria das distribuições, Laurent Schwartz, matemático francês e ganhador da medalha Fields em 1950 por seus estudos nessa área, foi um grande divulgador de ideias que usavam conceitos de generalização. Em seus trabalhos, propôs generalizações de funções capazes de centralizar um grande número de processos de natureza distinta [1]. De modo mais amplo, a generalização de funções consiste em reunir em "famílias" funções que possuam certas características e propriedades em comum.

Uma generalização das funções logaritmo e exponencial, sob um parâmetro, foi apresentada no fim da década de 1980, por Tsallis [2], no contexto da entropia não-extensiva. Tais generalizações possuem aplicações em vários campos da ciência, como por exemplo, em modelos discretos de crescimento e dinâmica de populações [3, 4], na psicofísica [5, 6], na espectometria [7] em distribuição de probabilidade 
generalizadas e funções de erro [8], entre outros. Além disso, essas generalizações constituem importantes ferramentas na simplificação de notações e manipulações algébricas. Existem outras generalizações, com um, dois ou até três parâmetros [9-11] e até com duas variáveis $[12,13]$. Contudo, não trataremos destas neste trabalho.

Neste contexto, a generalização da função logaritmo natural, $\ln _{\lambda}(t)$, para $t>0$, não apresenta qualquer restrição a tudo que seja próprio da função tradicional: pode ser feita, por exemplo, utilizando uma família de hipérboles assimétricas, de forma a preservar a igualdade $\ln _{\lambda}(1)=0, \forall \lambda \in \mathbb{R}[14]$. Por outro lado, a generalização da função exponencial $\mathrm{e}_{\lambda}(t)$, apresenta o fato de poder pertencer ao conjunto dos números complexos $\mathbb{C}$. Por essa razão defini-se $\mathrm{e}_{\lambda}(t)=0$, para $\lambda t<-1$. Isso, por um lado garante que $e_{\lambda}(t)$ tenha imagem real, por outro restringe a abrangência da generalização. Mais que isso, a extensão direta desta generalização para o plano complexo, mediante a troca de $t \in \mathbb{R}$ para $z \in \mathbb{C}$ no argumento principal, não mantém uma das mais importantes características de $\mathrm{e}^{z}, z \in \mathbb{C}$ : a analiticidade.

Em análise complexa, uma função $f$, definida em um conjunto aberto $D \subset \mathbb{C}$, é analítica em $z_{0} \in D$ se for diferenciável em $z_{0}$ e numa certa vizinhança deste ponto. Se $f$ for diferenciável em todo conjunto $D$, diz-se que ela é uma função holomorfa (ou analítica) em $D$. Este tipo de função carrega consigo uma série de características e propriedades que facilitam cálculos em vários aspectos e situações, principalmente no que se refere a integração. Ao integrar uma função analítica, evita-se estudos relativos a ramos e singularidades. Neste trabalho, nos dedicamos à busca de uma expressão analítica para a função exponencial generalizada no plano complexo e chegamos à seguinte relação:

$$
\mathrm{e}^{x+i y} \longrightarrow \mathrm{e}_{\lambda}(x)\left[\mathrm{e}_{\lambda}(y)\right]^{i}
$$

Com esta expressão, é possível estender a analiticidade da função exponencial na versão generalizada e, a partir daí, obter outras generalizações, tais como, das funções trigonométricas e hiperbólicas, da transformada de Fourier e das funções logperiódicas. Neste contexto, devido à recuperação da analiticidade, é possível dizer que uma generalização conveniente da função exponencial, com sua respectiva extensão para o plano complexo, colabora para que as demais generalizações sejam consistentes.

O Cap. 2 apresenta um breve histórico a respeito dessas funções e, como re- 
sultado, apresenta a extensão da função exponencial generalizada para o plano complexo, conforme já mencionado. Ao longo do texto, existe a discussão sobre quais expressões podem ser consideradas generalizações consistentes. Tal fato ocorre tanto no que se refere às funções exponencial e logaritmo generalizadas, como também em alguns operadores generalizados, onde damos sustentação teórica ao produto generalizado apresentado na Ref. [15]. Com este operador, apresentamos uma estrutura de anel generalizado, que garante a validade de muitas propriedades e teoremas próprios para esse tipo de estrutura algébrica [15-18]. Nessas discussões acerca de generalizações convenientes, destacamos três princípios básicos que devem ser preservados: características, propriedades e aplicabilidade. No que diz respeito ao terceiro item, verifica-se que a função logaritmo generalizado constitui uma ferramenta muito utilizada para transformação dados: a família de transformações Box-Cox [19].

No Cap. 3, apresentamos um apanhado histórico destas transformações seguindo as cronologias apresentadas por Sakia [20] e Daimon [21]. Apesar de anterior ao estudo de Tsallis [2], que enfatiza a conveniência em se generalizar a função logaritmo no contexto da física estatística, a primeira transformação de Box-Cox é a própria função logaritmo generalizado e, desta forma, as transformações desta "família" (que vieram posteriormente) podem ser escritas utilizando esta função. A representação dessas transformações em termos da função log-generalizada abre espaço para observar uma interessante curiosidade à respeito da transformação de Yeo e Johnson [22].

Outra generalização proposta é a da transformada de Fourier. No espírito de encontrar e verificar generalizações consistentes, o Cap. 4 faz um breve resumo das propriedades da transformada usual e destaca que ela é uma transformada integral e, portanto, possui uma característica crucial: ser linear. Mostramos que nossa proposta de transformada é linear e possui núcleo que permite generalizar várias propriedades equivalentes à da transformada usual, pois é possível obter a própria transformada original via um mapa conforme. Sendo assim, tal generalização segue os princípios de preservar características e propriedades, vitais para uma generalização consistente. A obtenção desta nova transformada só foi possível tendo em vista a extensão da função exponencial generalizada para o plano complexo, proposta no Cap. (2). Discutimos e ampliamos propriedades da nova generalização que 
propusemos na Ref. [23]. No que se refere à aplicação, utilizando essa generalização é possível reescrever a distribuição delta de Dirac. Além disso, apresentamos um exemplo em que a generalização é conveniente diante da transformada original de um sinal log-periódico, já que a original não consegue captar a significância do parâmetro $\omega_{0}$ ao transformar a função (log-periódica) $f(t)=\cos \left[\omega_{0} \ln (t)\right]$.

Funções log-periódicas são aquelas que possuem periodicidade na variável $\ln (t)$ ao invés de $t$. Elas são utilizadas em trabalhos que envolvem fraturas de materiais, análise de mercado financeiro [24, 25], previsão de terremotos [26-28], entre outros $[29,30]$. O Cap. 5 generaliza essas funções a partir do núcleo da generalização da transformada de Fourier apresentada no Cap. 4 e na Ref. [23]. A partir deste resultado, é possível obter generalizações para as funções trigonométricas e hiperbólicas, sempre levando em conta a discussão acerca de generalizações consistentes. Verificamos que a primeira generalização proposta por Borges [31] não preserva importantes características deste tipo de função, já que não oscilam e não são limitadas para qualquer valor do parâmetro $\lambda$. Em contrapartida, nossa proposta, obtida a partir do núcleo da transformada de Fourier generalizada, preserva estas características e estende outras propriedades equivalentes a das funções originais, tais como o limite fundamental e a relação fundamental da trigonometria. Mais uma vez, a analiticidade de $\mathrm{e}_{\lambda}(t)$, que propusemos, no plano complexo foi fundamental para a validade desse resultado.

O Cap. 6 traz as considerações sobre a conveniência da nossa proposta de função exponencial generalizada para argumentos complexos $\mathrm{e}^{x+i y} \longrightarrow \mathrm{e}_{\lambda}(x)\left[\mathrm{e}_{\lambda}(y)\right]^{i}$. Apresentamos também as discussões finais e perspectivas. 


\section{2}

\section{Funções e Operadores Generalizados}

O tipo de conhecimento que é sustentado apenas por observações e ainda não foi provado deve ser cuidadosamente diferenciado da verdade; é obtido por indução, como costumamos dizer. No entanto, vimos casos em que a mera indução levou ao erro.

A transformação de dados é um tema recorrente em análise estatística. Neste contexto, a transformação de Box-Cox [19] sempre se mostrou eficiente ao tomar um conjunto de dados $X=\left\{x_{1}, x_{2}, \cdots, x_{n}\right\}$ e, pela transformação

$$
y_{i}=\frac{x_{i}^{\lambda}-1}{\lambda},
$$

apresentar um novo conjunto de dados $Y=\left\{y_{1}, y_{2}, \cdots, y_{n}\right\}$ normalmente distribuídos, mediante um valor do parâmetro $\lambda$ adequado. Nota-se que essa transformação pode ser descrita com a função logaritmo generalizado, apresentada por Constantino Tsallis [2] no final da década de 1980. Tsallis apresentou a generalização da função logarítmica por

$$
\ln _{q}(t)=\lim _{q^{\prime} \rightarrow q} \frac{t^{1-q^{\prime}}-1}{1-q^{\prime}} .
$$

Ajustando $\lambda=1-q$ no limite da Eq. (2.2), obtém-se a transformação de Box-Cox da Eq. (2.1). Desta forma, por uma questão de clareza, manteremos neste texto, de agora em diante, as generalizações sempre no parâmetro $\lambda$, de modo que

$$
\ln _{\lambda}(t)=\lim _{\lambda^{\prime} \rightarrow \lambda} \frac{t^{\lambda^{\prime}}-1}{\lambda^{\prime}}
$$


A inversa da função logaritmo generalizada, $\ln _{\lambda}(t)$, é a função exponencial generalizada, $\mathrm{e}_{\lambda}(t)$. Neste capítulo, apresentamos um breve histórico sobre estas funções, suas propriedades e relações com os operadores generalizados. Apresentamos também uma extensão natural de $e_{\lambda}(t)$ para o plano complexo, mantendo as principais características da função original. Ao longo do texto, existe a discussão sobre como classificar uma generalização como consistente. Isso se faz presente tanto no que se refere às funções generalizadas, quanto aos operadores.

\subsection{Caminhos para generalização}

As funções logaritmo e exponencial são fundamentais para a ciência de modo geral. Encontrar generalizações para tais funções mediante um parâmetro $\lambda$ abre espaço para novas interpretações, novos estudos e novos tratamentos, além de ajudar na simplificação de expressões e nas manipulações algébricas. Para que a generalização se torne interessante, é necessário que uma família de funções possua uma forte relação com a função original. Isso significa que, a generalização, além de ter a função original como um caso limite para um determinado valor do parâmetro $\lambda$, deve ainda manter características essenciais destas funções e estender suas propriedades.

Neste contexto, é possível verificar generalizações bastante consistentes. Tsallis [2] apresentou sua versão para a generalização baseado em argumentos da termoestatística não extensiva. Já Arruda et al. [14] e Gonzalez [32], obtém a mesma generalização partindo de argumentos puramente geométricos. Estes consideraram inicialmente a família de funções $f_{\lambda}: \mathbb{R}_{+}^{*} \rightarrow \mathbb{R}, \lambda \in \mathbb{R}$ dada por:

$$
f_{\lambda}(t)=\frac{1}{t^{1-\lambda}} .
$$

Neste conjunto de funções, obtém-se, para $\lambda=1$, por exemplo, a função constante $f_{1}(t)=1$. A Fig. 2.1(a) traz outros exemplos de curvas pertencentes a esta família. Repare que, para $\lambda>1$, aparecem curvas conforme leis de potência e para $\lambda<$ 1, hipérboles. Especificamente para $\lambda=0$ é possível verificar que a hipérbole é simétrica em relação a bissetriz $f_{2}(t)=t$.

A "família" de funções inversa a $f_{\lambda}(t)$, indicada por $f_{\lambda}^{-1}(t)$, é apresentada como

$$
f_{\lambda}^{-1}(t)=\frac{1}{t^{\frac{1}{1-\lambda}}}
$$



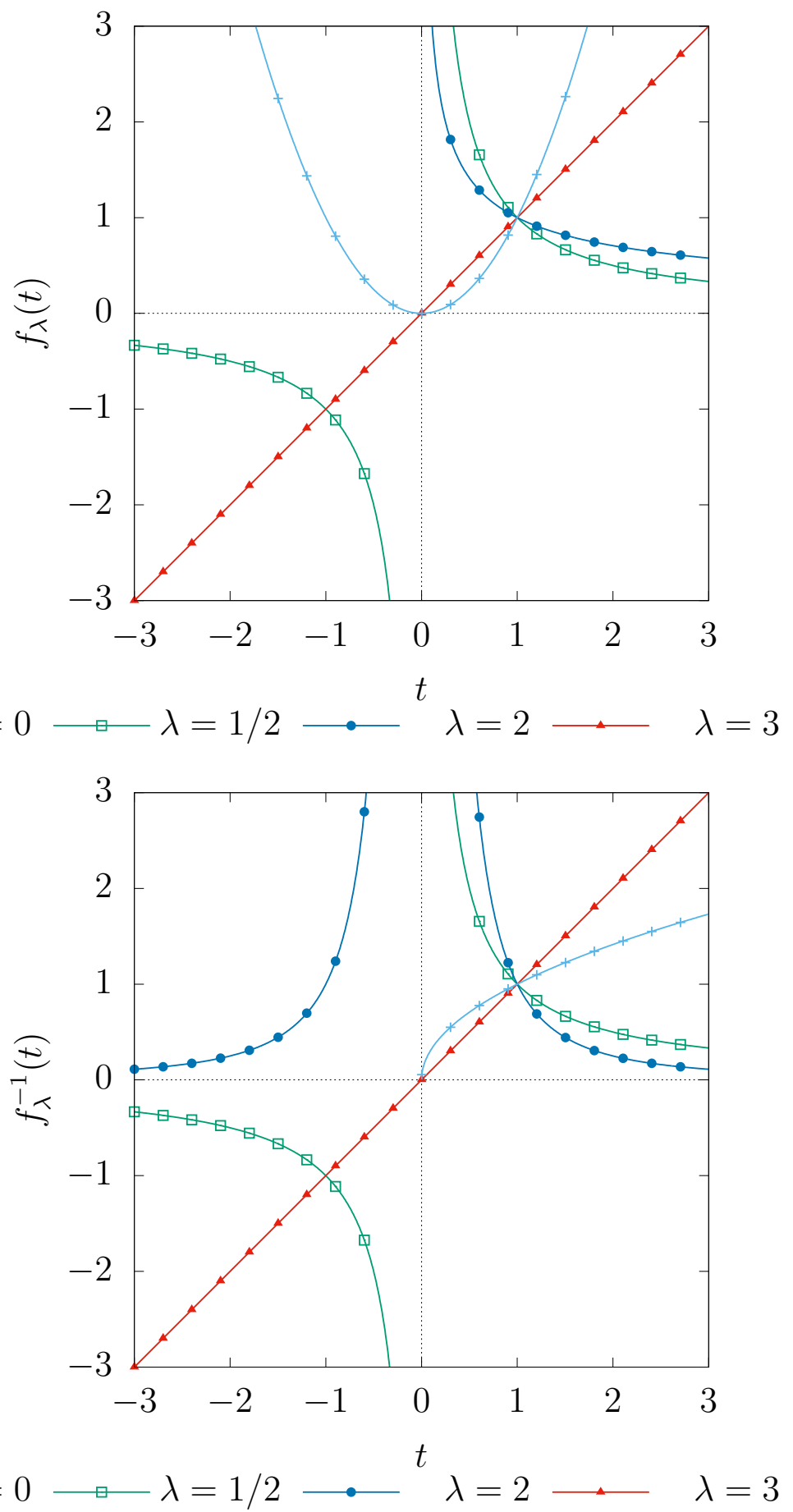

Figura 2.1: (a)Família de funções $f_{\lambda}(t)$ para valores de $\lambda$ iguais a $0,1 / 2,2$ e 3 . Note que $f_{\lambda}(t)$ tem comportamento hiperbólico para $\lambda<1$ e de lei de potência para $\lambda>1$. (b)Família de funções $f_{\lambda}^{-1}(t)$ para valores de $\lambda$ iguais a $0,1 / 2,2$, e 3 . Para $\lambda=2$ temos a função identidade $f_{2}^{-1}(t)=t$. Para $\lambda<2$ o comportamento é hiperbólico. Já para $\lambda>2$ temos leis de potência. 
O comportamento desta família de funções pode ser observado na Fig. 2.1(b) que traz alguns exemplos de curvas pertencentes a ela. Neste caso, a função não é definida quando $\lambda=1$. Se $\lambda<2$, tem-se um comportamento hiperbólico e para $\lambda>2$, leis de potência. Outra observação pertinente na Fig. 2.1 é que $f_{1 / 2}^{-1}(t)=f_{-1}(t)$. Em geral, $f_{\lambda}(t)=f_{\frac{\lambda}{\lambda-1}}^{-1}(t)$.

O fato de que tanto $f_{\lambda}(t)$ quanto sua inversa possuem comportamento hiperbólico para certos valores de $\lambda$ ou de leis de potência para outros, mostra uma tendência de continuidade e diferenciabilidade de cada uma dessas curvas, pelo menos em algumas regiões, o que será de importância no que segue.

\subsection{Função logaritmo generalizado}

Dadas as características mencionadas de $f_{\lambda}(t)$, é possível calcular as áreas sob cada curva e, assim, de maneira geométrica, integrar essa "família" de funções em determinadas regiões para qualquer valor de $\lambda$. Chamamos o resultado dessa integração [32, 33] de função logaritmo natural generalizada, que é equivalente à Eq. (2.3),

$$
\ln _{\lambda}(t)=\int_{1}^{t} f_{\lambda}\left(t^{\prime}\right) d t^{\prime}=\lim _{\lambda^{\prime} \rightarrow \lambda} \frac{t^{\lambda^{\prime}}-1}{\lambda^{\prime}}
$$

Da Eq. (2.6), é possível observar que se trata da definição de Leonhard Euler (1730) para a função logaritmo natural com a diferença de que o parâmetro $\lambda^{\prime}$ pode assumir qualquer valor $\lambda$, ao passo que, na definição original, $\lambda^{\prime} \rightarrow 0$. Desta forma, obtemos o logaritmo natural para $\lambda=0, \ln _{0}(t)=\ln (t)$. Graficamente, o comportamento desta generalização pode ser observado na Fig. 2.2. Para $\lambda=0$, obtém-se a função $\ln _{0}(t)=\ln (t)$, para $\lambda=1$, obtém-se a função afim $\ln _{1}(t)=$ $t-1$; para $\lambda=3$ a função $\ln _{3}(t)=\left(t^{3}-1\right) / 3$ e para $\lambda=-1$, obtém-se a função $\ln _{-1}(t)=(t-1) / t$ e assim por diante. À medida que $\lambda \rightarrow \infty$ é possível observar algo interessante: $\ln _{\infty}(t)$ tem o comportamento de função degrau (Heaviside) [34], conforme ilustrado na Fig. 2.3. No intervalo $-1 \leq t \leq 1$, tem-se $\lim _{\lambda \rightarrow \infty} \ln _{\lambda}(t)=$ 0. A Fig. 2.3 mostra o comportamento dessa função com alguns valores de $\lambda$ nas redondezas de 1 (Fig. 2.3a e -1 (Fig. 2.3b, para o caso de $\lambda$ par). Estes gráficos mostram que, no intervalo indicado, a função de fato tem um valor constante e este valor tende a zero à medida que $\lambda$ tende a infinito. Se $t>1, \lim _{\lambda \rightarrow \infty} \ln _{\infty}(t) \rightarrow \infty$. O 


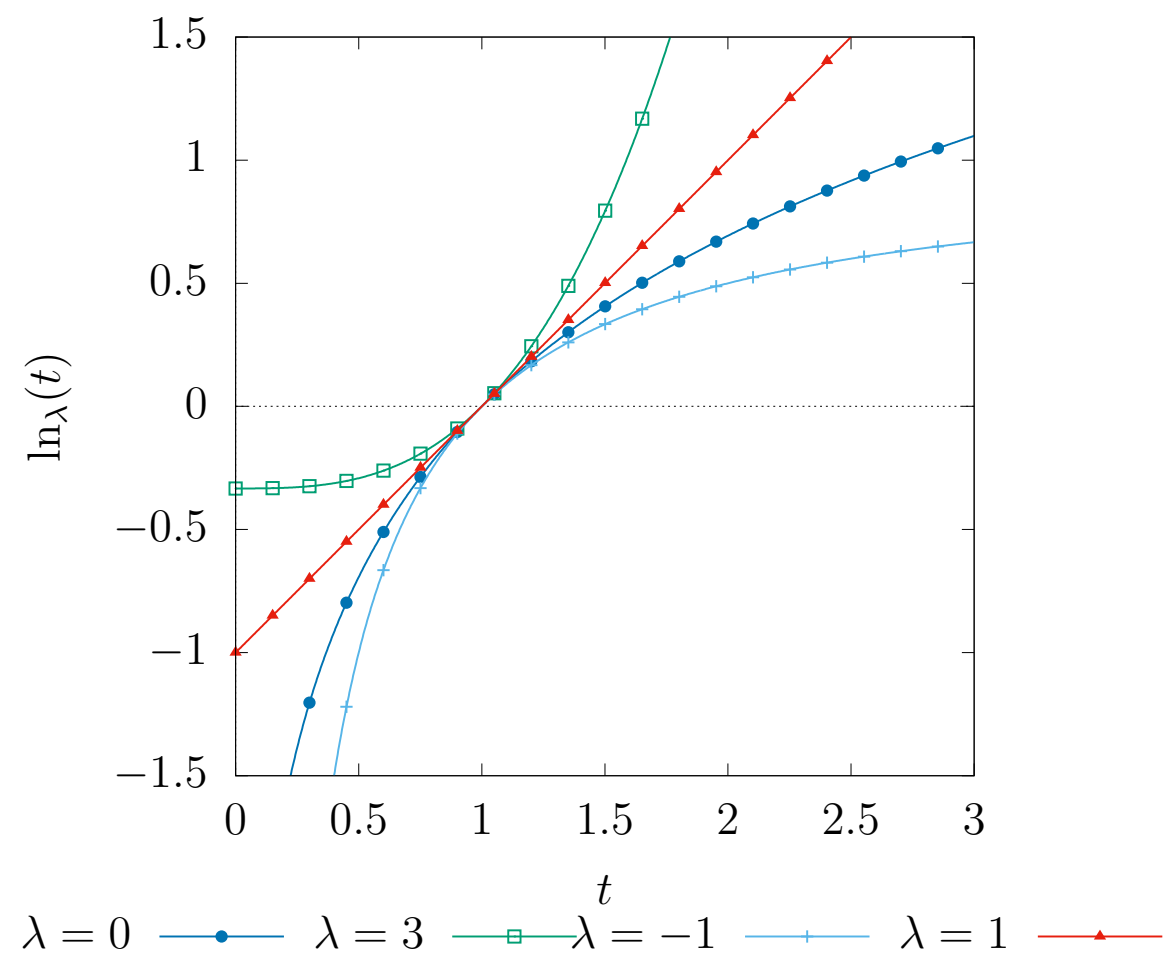

Figura 2.2: Função logaritmo generalizado $\ln _{\lambda}(t)$ para os valores de $\lambda$ iguais a $-1,0,1$, e 3 . Curvas mantém importantes características da função $\ln (t)$ tradicional, tais como, ser estritamente negativa para os valores de $t<1$, nula em $t=1$ e positivas para $t>1$. É possível observar ainda uma simetria de inversão $\lambda_{N}=2-\lambda$.

mesmo ocorre quando $t<-1$ e $\lambda \rightarrow \infty$, com $\lambda$ par Fig (2.3 a). Já para $t<-1$, $\lambda \rightarrow \infty$, com $\lambda$ impar, então $\lim _{\lambda \rightarrow \infty} \ln _{\infty}(t) \rightarrow-\infty$ Fig (2.3 b).

Partindo da função original, pode-se verificar que muitas propriedades e características do $\ln (t)$ estão presentes também nesta generalização $\ln _{\lambda}(t)$. Um bom exemplo é extraído do gráfico apresentado na Fig. 2.2: todas as curvas cortam o eixo das abcissas em $t=1$, pois $\ln _{\lambda}(1)=0, \forall \lambda \in \mathbb{R}$. Além disso, analisando a Eq. (2.6), é possível observar que a integral é considerada negativa no intervalo $0<t<1$ e positiva para $t>1$, para todas as curvas, o que também é próprio da função logaritmo natural.

Outras propriedades também podem ser observadas nesta generalização, como por exemplo, a simetria em relação à bissetriz ímpar, de tal forma que $\lambda_{N}=2-\lambda$. Outro exemplo de propriedade pode ser observado tomando-se uma deformação $\alpha \neq 0$. Nestes termos, teremos que

$$
\ln _{\lambda}\left(t^{\alpha}\right)=\frac{\left(t^{\alpha}\right)^{\lambda}-1}{\lambda}=\alpha\left[\frac{(t)^{\alpha \lambda}-1}{\alpha \lambda}\right]=\alpha \ln _{\alpha \lambda}(t) .
$$



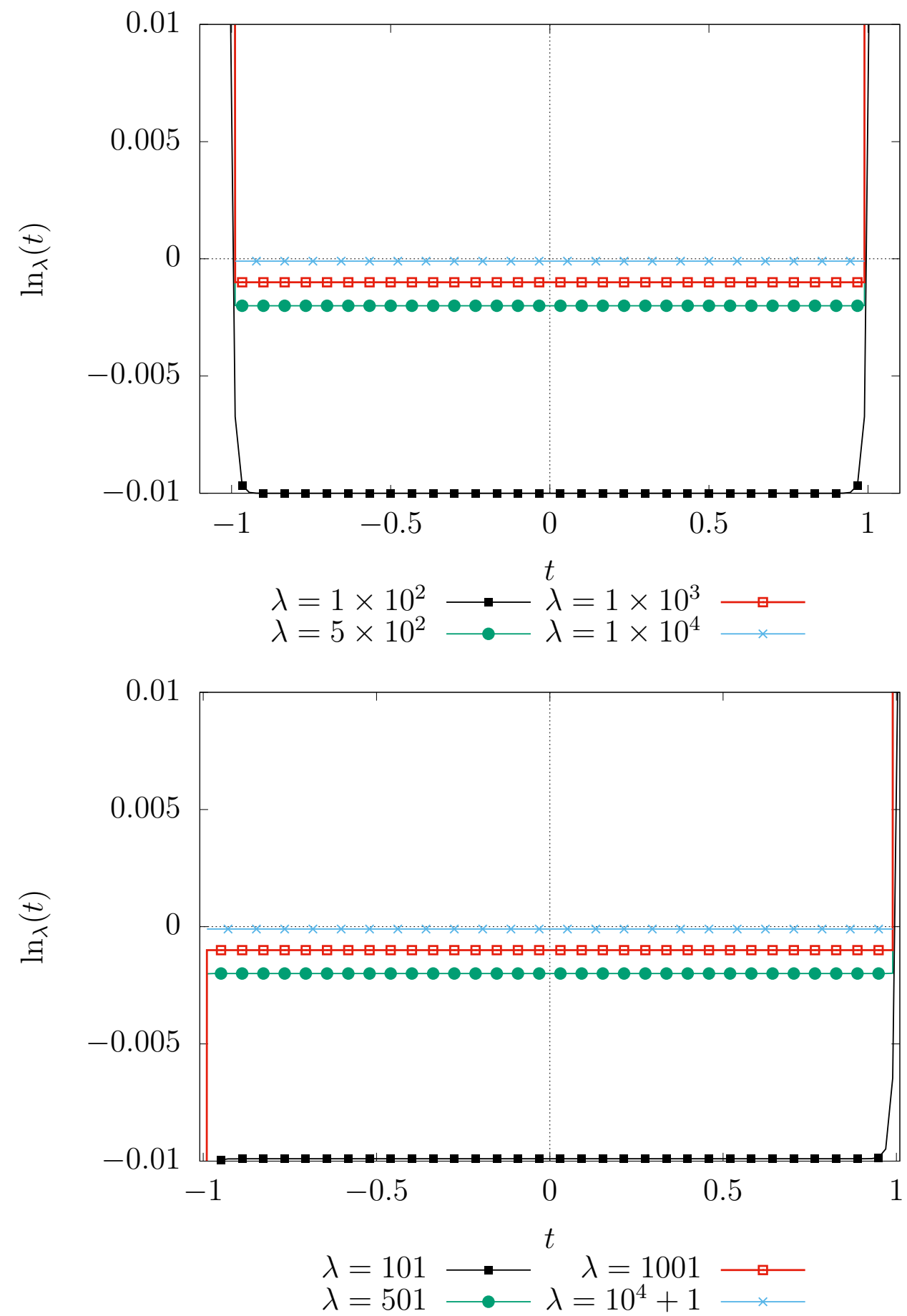

Figura 2.3: (a)Função logaritmo generalizado com parâmetro par: $\lambda=2 n, n \rightarrow \infty$. Neste caso a função se configura como degrau com $\ln _{\infty}(t)=0$ no intervalo $-1 \leq t \leq 1$ e $\ln _{\infty}(t)=\infty$ fora dele.(b) Função logaritmo generalizado com parâmetro ímpar: $\lambda=2 n-1, n \rightarrow \infty$. Neste caso a função também é Heaviside com praticamente as mesmas características do caso anterior, porém no intervalo $t<1, \ln _{\infty}(t)=-\infty$. 
De modo particular, para $\alpha=-1$ obtém-se $\ln _{\lambda}\left(t^{-1}\right)=-\ln _{-\lambda}(t)$. Para $\lambda=0$, e $\alpha$ qualquer, recuperamos a conhecida propriedade $\ln \left(t^{\alpha}\right)=\alpha \ln (t)$. Como consequência desta propriedade temos

$$
\ln _{\lambda}(t)+\ln _{\lambda}(1 / t)=\frac{\lambda}{t^{\lambda}}\left[\ln _{\lambda}(t)\right]^{2} .
$$

Para $\lambda=0$, obtém-se o conhecido resultado $\ln (t)+\ln (1 / t)=0, \forall t \in \mathbb{R}_{+}$da função convencional.

No que diz respeito à derivada da função $\ln _{\lambda}(t)$, pode-se utilizar a Eq.(2.4) e o teorema fundamental do cálculo para afirmar que

$$
\frac{\mathrm{d}}{\mathrm{d} t} \ln _{\lambda}(t)=t^{\lambda-1}=f_{\lambda}(t)
$$

de tal sorte que, quando $\lambda=0$ obtém-se $\frac{\mathrm{d} \ln _{0}(t)}{\mathrm{d} t}=t^{-1}$, a conhecida derivada do logaritmo natural. Por estes mesmos mecanismos chegamos que

$$
\int \ln _{\lambda}(t) d t=\frac{t}{1+\lambda}\left[\ln _{\lambda}(t)-1\right]+C
$$

com $C$ constante de integração. Novamente, quando $\lambda=0$, reobtemos $t(\ln (t)-1)+$ $C$, a integral da função $\ln (t)$ convencional.

Dentro de sistemas dinâmicos, a separação de escalas entre variáveis que mudam rapidamente e outras que mudam lentamente, dá origem aos conceitos de variáveis rápidas e variáveis lentas [35]. Ao tomar, por exemplo, duas variáveis temporais, $x$ medida em dias e $y$ em semanas, tem-se que $x$ pode ser considerada uma variável rápida e $y$ uma variável lenta. No que diz respeito à função $\ln _{\lambda}(t)$, pode-se dizer que, em contraposição com a variável rápida $t$, o parâmetro $\lambda$ pode ser considerado como uma variável lenta e, neste caso, podemos realizar outros estudos. Por exemplo, se na transformação de Box-Cox for necessário a otimização de $\lambda$ com a finalidade de verificar qual valor torna um determinado conjunto de dados mais próximo de uma distribuição normal pelo princípio da máxima verossimilhança. Neste caso, encontrar a derivada nesta variável pode ser um recurso precioso, assim

$$
\frac{\mathrm{d}}{\mathrm{d} \lambda} \ln _{\lambda}(t)=\frac{1}{\lambda}\left[t^{\lambda} \ln (t)-\ln _{\lambda}(t)\right]
$$

Por outro lado, integrando a função $\ln _{\lambda}(t)$ em relação ao parâmetro $\lambda$ obtemos como resultado

$$
\int \ln _{\lambda}(t) d \lambda=-\ln (\lambda)-\gamma[0,-\lambda \ln (t)]+C
$$


onde $\gamma(a, b)$ é a função "gama incompleta" [34] e $C$ uma constante.

Além das propriedades e características apresentadas acima, existem outras que são válidas na definição usual de forma que suas validades seriam desejáveis para a generalização. Uma dessas propriedades é a que relaciona somas de logaritmos,

$$
\ln (a)+\ln (b)=\ln (a b)
$$

Não é possível obter uma relação deste tipo com as operações convencionais de adição e multiplicação, já que $\ln _{\lambda}(a)+\ln _{\lambda}(b) \neq \ln _{\lambda}(a b)$. Desta forma, existe a necessidade de outros operadores que mantenham a validade para caso tradicional e que, ao mesmo tempo, seja válido para o caso generalizado, como apresentado na próxima seção.

\subsection{Operações ou operadores binários}

Operações binárias (ou operadores) são um tema recorrente a estudos matemáticos das áreas de Álgebra e Teoria dos Números. Estas operações já fazem parte do cotidiano da humanidade há muito tempo. Algumas operações, como a soma por exemplo, surgiram mesmo antes da escrita formal [36].

Uma operação binária $\varphi$ é uma aplicação em um conjunto $A$, não vazio, também chamada de lei de composição interna em $A$ [37], de tal forma que

$$
\varphi: A \times A \rightarrow A .
$$

Neste caso, dados $a, b \in A$, a operação $\varphi$ é indicada por $a \varphi b$. Na prática, utilizamse símbolos ao invés de letras gregas. É interessante perceber que, por exemplo, a subtração não é uma operação dentro do conjunto dos números naturais, pois, dado $3 \in \mathbb{N}$ e $5 \in \mathbb{N}, 3-5 \notin \mathbb{N}$.

Existem algumas propriedades notáveis às operações. Desta forma, dada uma operação $*$, definida num conjunto não vazio $A$, tem-se que ela é:

1. Comutativa se

$$
\forall a, b \in A, a * b=b * a ;
$$

2. Associativa se

$$
\forall a, b, c \in A,(a * b) * c=a *(b * c) ;
$$


3. Possui um elemento neutro $e \in A$, se

$$
\forall a \in A, a * e=e * a=a .
$$

O elemento neutro, quando existe, é único.

4. Possui um elemento nulo $\phi \in A$, se

$$
\forall a \in A, a \neq \phi, a * \phi=\phi * a=\phi .
$$

5. Possui inverso se possuir elemento neutro e dado $a \in A$ existir $a^{\prime} \in A$ de tal forma que

$$
a * a^{\prime}=a^{\prime} * a=e .
$$

6. Possui integridade se

$$
\forall a, b \in A, a * b=0_{A} \Rightarrow a=0_{A} \text { ou } b=0_{A},
$$

em que $0_{A}$ é o "zero" do conjunto (ou espaço) $A$.

Além dessas propriedades, a propriedade distributiva é de suma importância. Dadas $*$ e $\circledast$, duas operações em $A$. A operação $*$ é distributiva em relação à $\circledast$ se

$$
\begin{aligned}
& a *(b \circledast c)=(a * b) \circledast(a * c) \\
& (a \circledast b) * c=(a * c) \circledast(b * c)
\end{aligned}
$$

As propriedades apresentadas são facilmente reconhecidas na adição e multiplicação dos números reais ou complexos, por exemplo. Contudo, elas possuem uma importância muito maior na Álgebra. Uma operação * em um conjunto não vazio $A$ e este mesmo conjunto constituem uma estrutura algébrica. As estruturas mais conhecidas são os Grupos, os Anéis e os Corpos. Por exemplo, a estrutura $(A, *)$ é um Grupo se a operação * for associativa, possuir elemento neutro e também elemento inverso. Se além dessas propriedades * também for comutativa, então a estrutura $(A, *)$ é conhecida como Grupo abeliano. Um outro exemplo é o de uma estrutura que conta com um conjunto e duas operações: $(A, *, \circledast)$. Tal estrutura constitui um anel se $(A, *)$ for um grupo abeliano e a operação $\circledast$ for associativa e distributiva em relação a $*$. Conforme a operação $\circledast$ tem validade de outras propriedades, esse anel recebe nomenclaturas especiais, à saber: 
1. Anel comutativo, se $\circledast$ for comutativa;

2. Anel com unidade, se $\circledast$ possuir elemento neutro;

3. Anel de integridade, se $\circledast$ for comutativa, possuir elemento neutro unitário e for de integridade;

4. Anel com divisão, se $\circledast$ possuir inverso para cada elemento $a \in A$.

Um corpo é um anel $(A, *, \circledast)$ comutativo e com divisão. O conjunto dos números reais munido das operações adição $(+)$ e multiplicação $(\cdot)$ habituais constitui um corpo $(\mathbb{R},+, \cdot)$. A vantagem de se encontrar uma dessas estruturas é que existem muitas outras propriedades já demonstradas, válidas especificamente para cada uma, o que acaba facilitando nossos estudos.

\subsection{Operadores generalizados}

$\mathrm{Na}$ busca de argumentos para validar a propriedade da soma de logaritmos na versão generalizada, Borges[38] e Nivanen[39] apresentam um operador binário, denominado "soma generalizada", com a seguinte descrição:

$$
a \oplus_{\lambda} b=a+b+\lambda a b .
$$

Este operador retoma a soma habitual quando $\lambda=0$, ou seja, $a \oplus_{0} b=a+b$ e mantém válida algumas importantes propriedades da soma comum:

1. Comutatividade: $a \oplus_{\lambda} b=b \oplus_{\lambda} a$;

2. Associatividade: $\left(a \oplus_{\lambda} b\right) \oplus_{\lambda} c=a \oplus_{\lambda}\left(b \oplus_{\lambda} c\right)$;

3. Zero como elemento neutro: $a \oplus_{\lambda} 0=0 \oplus_{\lambda} a=a$;

4. Elemento inverso: $-a /(1+\lambda a)$, já que $a \oplus_{\lambda}[-a /(1+\lambda a)]=0, \forall \lambda, a \in \mathbb{R}$;

De posse deste operador, é possível apresentar uma propriedade geral na qual a soma de logaritmos tradicionais tenha validade:

$$
\ln _{\lambda}(a) \oplus_{\lambda} \ln _{\lambda}(b)=\ln _{\lambda}(a b)
$$

Nesta expressão, quando $\lambda=0$, retomamos a propriedade do logaritmo usual apresentada na Eq.(2.13). 
Uma outra característica deste operador é ser não distributivo em relação à multiplicação tradicional, dado que $a\left(b \oplus_{\lambda} c\right) \neq a b \oplus_{\lambda} a c$. Tal propriedade é desejável pois abre a possibilidade de criarmos uma estrutura de anel dentro do contexto dos "operadores generalizados".

$\mathrm{Na}$ álgebra tradicional, uma estrutura de anel consiste em um conjunto numérico e duas operações binárias fechadas nele, uma usualmente chamada de soma e a outra produto, conforme apresentado na Sec. 2.3. O fato de encontrar uma estrutura deste tipo traria segurança e validade de muitas propriedades já demonstradas. Convém portanto, buscar alternativas que deixem aberta essa possibilidade de estrutura.

Com o intuito de validar propriedades importantes das funções logaritmos no contexto generalizado, Borges [38] e Nivanen[39] apresentam ainda uma outra forma de relacionar a soma de dois logaritmos generalizados. Levando-se em conta que $\ln _{\lambda}(a)+\ln _{\lambda}(b)=\ln _{\lambda}\left[\left(a^{\lambda}+b^{\lambda}-1\right)^{1 / \lambda}\right]$ e que, além disso, $\lim _{\lambda \rightarrow 0}\left(a^{\lambda}+b^{\lambda}-1\right)^{1 / \lambda}=a b$, pode-se definir um outro operador

$$
a \otimes_{\lambda} b=\left(a^{\lambda}+b^{\lambda}-1\right)^{1 / \lambda}
$$

chamado de produto generalizado. Este operador, quando $\lambda=0$, retorna à multiplicação tradicional, ou seja, $a \otimes_{0} b=a b$. Além disso, este operador possui as seguintes propriedades:

1. Comutatividade: $a \otimes_{\lambda} b=b \otimes_{\lambda} a$;

2. Associatividade: $\left(a \otimes_{\lambda} b\right) \otimes_{\lambda} c=a \otimes_{\lambda}\left(b \otimes_{\lambda} c\right)$;

3. Elemento Neutro unitário: $a \otimes_{\lambda} 1=1 \otimes_{\lambda} a=a$;

4. Elemento inverso: $a^{-1}=\left(2-a^{\lambda}\right)^{1 / \lambda}$, já que $a \otimes_{\lambda}\left(2-a^{\lambda}\right)^{1 / \lambda}=\left(2-a^{\lambda}\right)^{1 / \lambda} \otimes_{\lambda} a=1$.

Quanto ao elemento inverso, deve-se destacar que ele é muito restrito, já que para valores de $a^{\lambda}>2$ e $\lambda$ par, $\left(2-a^{\lambda}\right)^{1 / \lambda} \notin \mathbb{R}$. Outra característica deste operador é que não é possível validar a propriedade distributiva, nem em relação à soma tradicional, já que $a \otimes_{\lambda}(b+c) \neq a \otimes_{\lambda} b+a \otimes_{\lambda} c$, e tão pouco quanto à soma generalizada, já que $a \otimes_{\lambda}\left(b \oplus_{\lambda} c\right) \neq\left(a \otimes_{\lambda} b\right) \oplus_{\lambda}\left(a \otimes_{\lambda} c\right)$.

Outra questão que deve ser levantada a respeito deste operador é relativa ao elemento nulo. Na multiplicação convencional temos que $a \cdot 0=0 \cdot a=0, \forall a, b$. 
Neste produto generalizado, $a \otimes_{\lambda} 0=\left(a^{\lambda}-1\right)^{1 / \lambda}$ ou seja, para que este produto seja zero $\left(a^{\lambda}-1\right)^{1 / \lambda}=0$ o que implica em $\lambda=0$ ou $a=1$. Por exemplo, se $\lambda=2$, quando operamos, $\left(x^{2}+1\right)^{1 / 2} \otimes_{\lambda} 0$ tem-se $\left\{\left[\left(x^{2}+1\right)^{1 / 2}\right]^{2}+0^{2}-1\right\}^{1 / 2}=\left(x^{2}+1-1\right)^{1 / 2}=$ $\left(x^{2}\right)^{1 / 2}= \pm x \neq 0$, se $x \neq 0$.

Uma alternativa a essa generalização do produto é apresentada por Lobão [15] e utilizada, mais tarde, por Kalogeropoulos [17, 18]. Nestas referências, o produto é definido por

$$
a \odot_{\lambda} b=\frac{1}{\lambda}\left[(1+\lambda)^{\frac{\ln [1+\lambda a] \cdot \ln [1+\lambda b]}{[\ln (1+\lambda)]^{2}}}-1\right] .
$$

Neste caso, apresenta-se uma generalização do produto que atende à propriedade distributiva, criando uma estrutura algébrica $\left(\mathbb{R}_{\lambda}, \oplus_{\lambda}, \odot_{\lambda}\right)$ que é, pelo menos, um anel de integridade para qualquer $\lambda[15-17]$. Também neste operador generalizado, quando $\lambda=0$ obtém-se o produto tradicional $a \odot_{0} b=a b$. Além disso, este operador possui as seguintes propriedades:

1. Comutatividade: $a \odot_{\lambda} b=b \odot_{\lambda} a$;

2. Associatividade: $\left(a \odot_{\lambda} b\right) \odot_{\lambda} c=a \odot_{\lambda}\left(b \odot_{\lambda} c\right)$;

3. Elemento neutro unitário: $a \odot_{\lambda} 1=1 \odot_{\lambda} a=a$;

4. Elemento inverso dado por

$$
a^{-1}=\frac{\mathrm{e}^{\frac{[\ln (1+\lambda)]^{2}}{\ln (1+\lambda a)}-1}}{\lambda},
$$

já que $a \odot_{\lambda} a^{-1}=a^{-1} \odot_{\lambda} a=1$.

5. Elemento nulo 0: $a \odot_{\lambda} 0=0 \odot_{\lambda} a=0, \forall \lambda, a \in \mathbb{R}$.

Outra importante constatação é que este operador generalizado é distributivo em relação à soma generalizada, ou seja, $a \odot_{\lambda}\left(b \oplus_{\lambda} c\right)=\left(a \odot_{\lambda} b\right) \oplus_{\lambda}\left(a \odot_{\lambda} c\right)$. Um problema deste operador é que não cumpre o papel de validar, de forma alguma, a relação entre soma de logaritmos e o logaritmo de um "produto", o que não é considerado capital, já que existiria pelo menos uma relação: a da soma generalizada.

Apresentamos, portanto, duas possibilidades de generalização para o produto. Ambas com muitas características próprias da multiplicação convencional, o que as sustenta muito bem dentro do contexto em que cada uma foi criada. Existe um "porém" em cada uma delas: a não distributividade da primeira proposta $[38,39]$ e 
a não relação com a soma de logaritmos na segunda [15]; tudo dentro do esperado e que não desmerece nenhuma das generalizações apresentadas.

Na álgebra tradicional, costuma-se apresentar a subtração (ou diferença) como operação inversa à soma. Desta forma, $a+(-a)=0$. No contexto generalizado observa-se que o inverso de um elemento a na soma é dado por $-a /(1+\lambda a)$, já que $a \oplus_{\lambda}[-a /(1+\lambda a)]=0$. De forma simplificada pode-se escrever $a \oplus_{\lambda}\left(\ominus_{\lambda} a\right)=0 \mathrm{e}$ definir o operador diferença generalizado como

$$
a \ominus_{\lambda} b=\frac{a-b}{1+\lambda b}
$$

que quando $\lambda=0$ retorna o operador diferença usual.

O operador diferença generalizado, a exemplo da versão tradicional, não é comutativa pois $a \ominus_{\lambda} b \neq b \ominus_{\lambda} a$ e não associativa pois $\left(a \ominus_{\lambda} b\right) \ominus_{\lambda} c \neq a \ominus_{\lambda}\left(b \ominus_{\lambda} c\right)$. Possui elemento neutro à direita, já que $a \ominus_{\lambda} 0=a$ e não à esquerda, já que ao operar o 0 à esquerda obtém-se o simétrico da soma generalizada pois $0 \ominus_{\lambda} a=\ominus_{\lambda} a$.

Pode-se utilizar a diferença generalizada para obter uma propriedade interessante dos logaritmos generalizados. Resgatando $\ln (a)-\ln (b)=\ln (a / b)$, temos, na versão generalizada

$$
\ln _{\lambda}(a) \ominus_{\lambda} \ln _{\lambda}(b)=\ln _{\lambda}\left(\frac{a}{b}\right)
$$

A operação inversa da multiplicação é a divisão. Na versão generalizada pode-se definir o operador divisão como:

$$
a \oslash_{\lambda} b=\left(a^{\lambda}-b^{\lambda}+1\right)^{\frac{1}{\lambda}}
$$

No limite de $\lambda \rightarrow 0$, obtem-se a divisão convencional $a / b$. É importante apontar que, a exemplo da divisão convencional, a generalizada também não é comutativa, pois $a \oslash_{\lambda} b \neq b \oslash_{\lambda} a$ e não é associativa já que $\left(a \oslash_{\lambda} b\right) \oslash_{\lambda} c \neq a \oslash_{\lambda}\left(b \oslash_{\lambda} c\right)$. Além disso, pode-se observar também que $a \oslash_{\lambda} 1=a$ e $1 \oslash_{\lambda} a=\oslash_{\lambda} a$, apresentado na Ref. [32]. Com esse operador generalizado é possível escrever mais uma relação de $\ln _{\lambda}(t)$ :

$$
\ln _{\lambda}\left(a \oslash_{\lambda} b\right)=\ln _{\lambda}(a)-\ln _{\lambda}(b)
$$

que quando $\lambda \rightarrow 0$ leva a $\ln (a / b)=\ln (a)-\ln (b)$.

Em relação ao operador produto apresentado como alternativo $\left(\odot_{\lambda}\right)$ na 
Eq. (2.26) a operação inversa é:

$$
a *_{\lambda} b=\frac{1}{\lambda}\left[(1+\lambda)^{\frac{\ln (1+\lambda a)}{\ln (1+\lambda b)}}-1\right] .
$$

Também nesta operação, quando $\lambda=0$ reobtemos a divisão tradicional. Além disso, esse operador carrega características próprias do quociente tradicional, já que não é comutativo pois $a *_{\lambda} b \neq b *_{\lambda} a$ e nem associativo já que $\left(a *_{\lambda} b\right) *_{\lambda} c \neq a *_{\lambda}\left(b *_{\lambda} c\right)$. Nota-se, ainda, que a exemplo da divisão comum, $a *_{\lambda} 1=a$ e $1 *_{\lambda} a$ é o elemento inverso do produto $\odot_{\lambda}$ da Eq. (2.26).

Com a discussão apresentada, é possível verificar que todas essas generalizações possuem características e propriedades significativamente próximas (e próprias) das operações originais, o que nos leva a entender que elas são bem definidas dentro do contexto em que foram criadas. Tem-se ainda que a estrutura $\left(\mathbb{R}, \oplus_{\lambda}, \odot_{\lambda}\right)$ constitui um anel de integridade para qualquer $\lambda \neq 0$ e a estrutura de corpo para $\lambda=0$, na verdade o já conhecido corpo dos números reais, munido das operações soma $(+)$ e multiplicação $(\cdot)$ habituais. Este fato garante a validade de muitas propriedades inerentes a estas estruturas, além de criar a expectativa de estudos sobre uma "Estrutura algébrica generalizada". No decorrer deste trabalho, iremos utilizar estes operadores.

\subsection{A função exponencial generalizada}

A família de funções $\ln _{\lambda}(t)$ apresentada na Eq. (2.3) é bijetora para cada

valor de $\lambda$, admitindo, portanto, inversa. Tal inversa é chamada de exponencial generalizada e pode ser escrita como

$$
\mathrm{e}_{\lambda}(t)= \begin{cases}\lim _{\lambda^{\prime} \rightarrow \lambda}\left(1+\lambda^{\prime} t\right)^{1 / \lambda^{\prime}} & , \text { se } \lambda^{\prime} t \geq-1 \\ 0 & , \text { caso contrário }\end{cases}
$$

É possível perceber que, de fato,

$$
\ln _{\lambda}\left[\mathrm{e}_{\lambda}(t)\right]=\frac{\left[\mathrm{e}_{\lambda}(t)\right]^{\lambda}-1}{\lambda}=\frac{\left[(1+\lambda t)^{1 / \lambda}\right]^{\lambda}-1}{\lambda}=\frac{[1+\lambda t-1]}{\lambda}=t .
$$

Por outro lado,

$$
\mathrm{e}_{\lambda}\left[\ln _{\lambda}(t)\right]=\left[1+\lambda \ln _{\lambda}(t)\right]^{1 / \lambda}=\left(t^{\lambda}\right)^{1 / \lambda}=t .
$$

A exemplo do logaritmo, quando se compara a generalização com a definição de Euler na função exponencial, percebe-se que a diferença está no fato de $\lambda^{\prime}$ poder 
assumir qualquer valor $\lambda$, enquanto na definição original $\lambda^{\prime} \rightarrow 0$ e, desta forma, $\mathrm{e}_{0}(t)=\mathrm{e}^{t}$

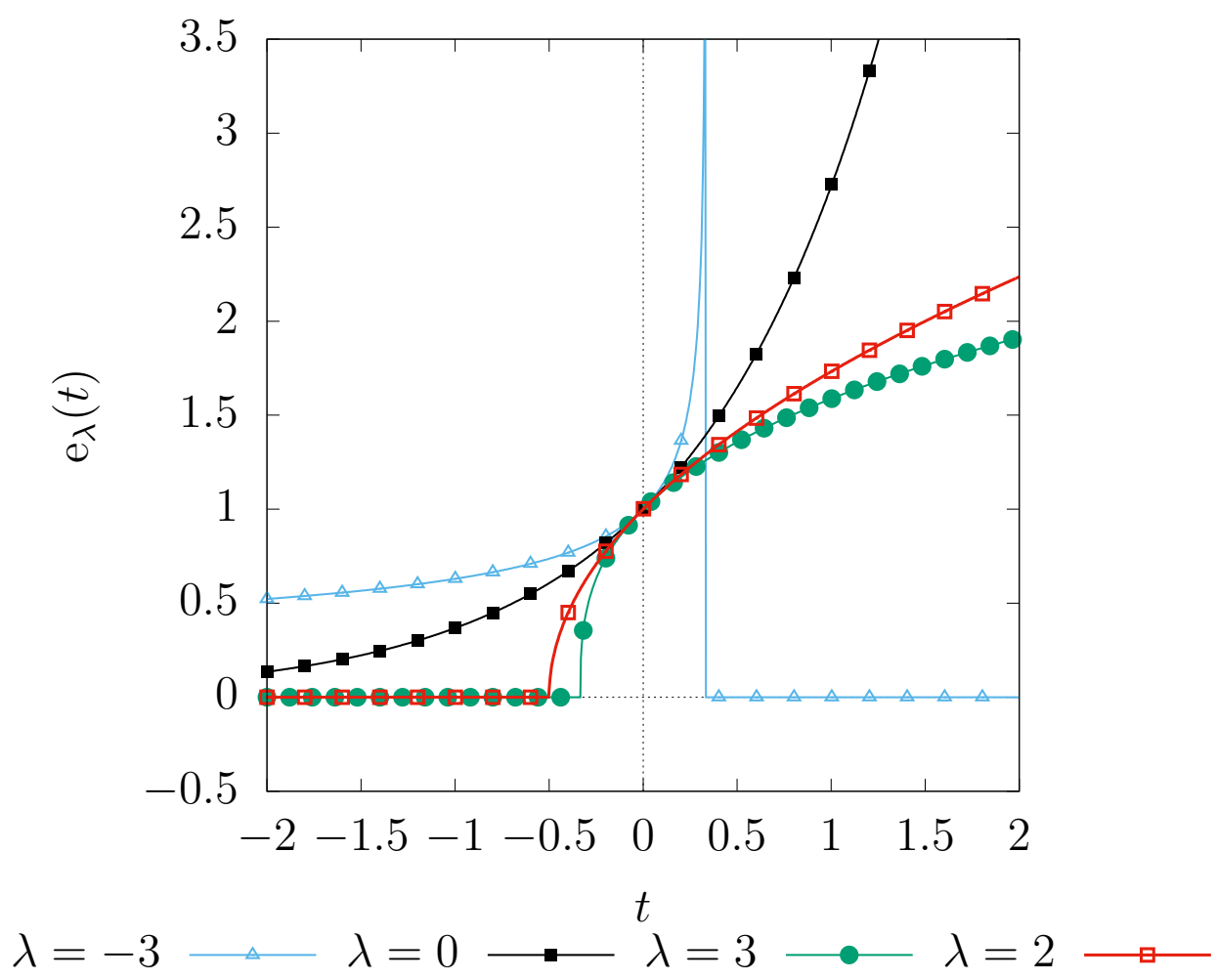

Figura 2.4: Função exponencial generalizada $\mathrm{e}_{\lambda}(t)$ e seus comportamentos para valores de $\lambda=-3$ : função hiper exponencial com divergência em $t=1 / 3 ; \lambda=0$ : exponencial convencional; $\lambda=2$ : função raiz quadrada; $\lambda=3$ : raiz cúbica.

O comportamento desta família de funções pode ser observado na Fig. 2.4. Para $\lambda=0$, observa-se a exponencial convencional. Para $\lambda=1$, a função afim $\mathrm{e}_{1}(t)=1+t$. Se $\lambda=2$, obtém-se a função raiz $\mathrm{e}_{2}(t)=\sqrt{1+2 t}$. Já para valores de $\lambda=n, n>2$ e inteiro, tem-se funções radicais de índice $n$. Se $n=3$, por exemplo, a função radical passa a ser $e_{3}(t)=\sqrt[3]{1+3 t}$ e assim por diante até que, para valores de $\lambda \rightarrow \infty$ a função $e_{\lambda}(t)$ tende a uma reta em $y=1$, o que é apresentado na Fig. 2.5. Para valores negativos de $\lambda$, vê-se crescimento hiper exponencial com divergências em $t=-1 / \lambda$. Para $\lambda=-3$, por exemplo, tem-se uma curva crescente que diverge em $t=1 / 3$, conforme ilustra a Fig. 2.4. Também é possível observar que a definição de $e_{\lambda}(t)$ possui um pequeno problema, que não chega a ser "capital" mas tira a "naturalidade" da mesma. O fato de exigir que $\mathrm{e}_{\lambda}(t)=0$ se $\lambda t<-1$, pode ser, em alguns casos, descritivos de transição de fase, como a transição para a 


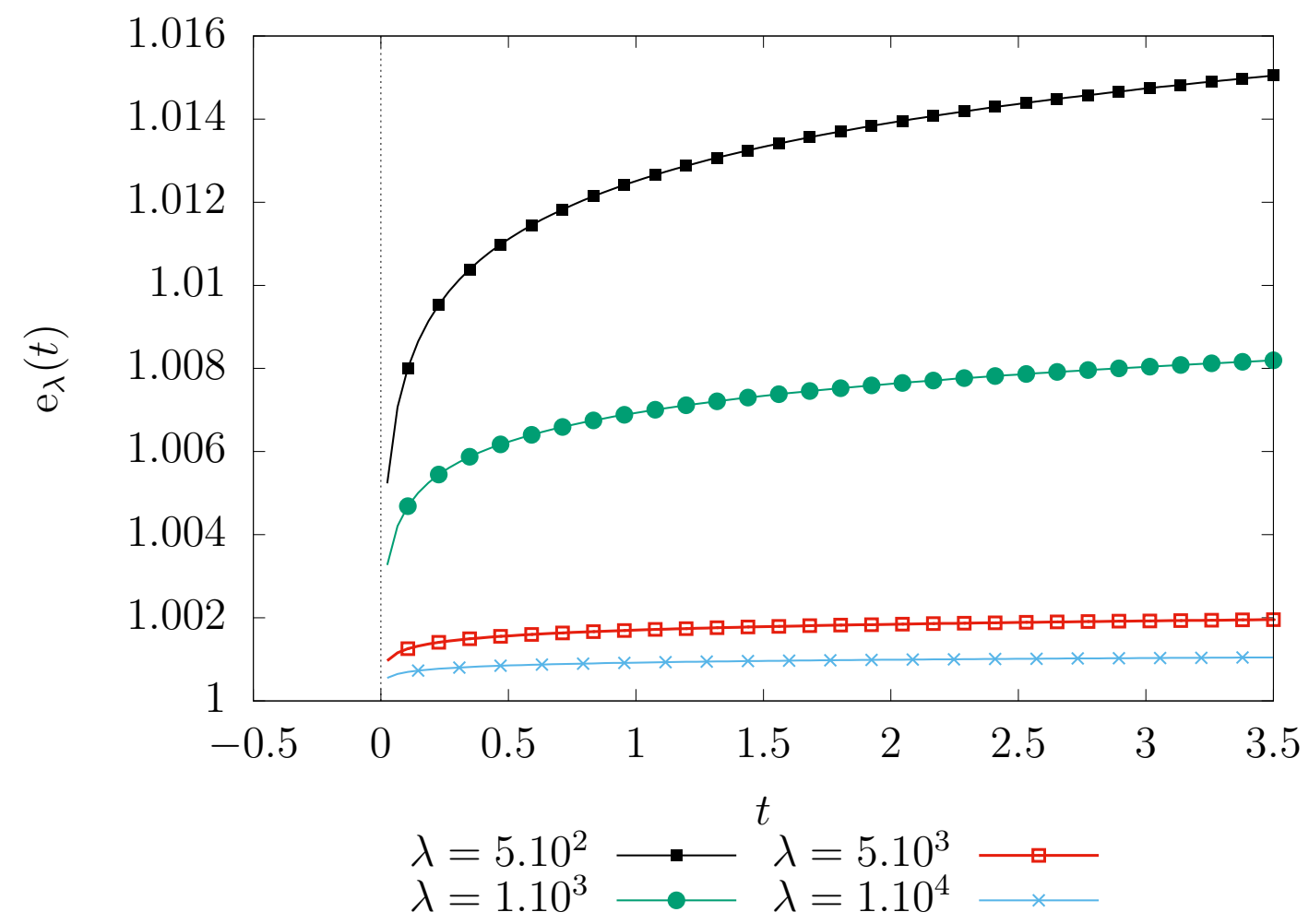

Figura 2.5: Função exponencial generalizada $e_{\lambda}(t)$ para valores "grandes" de $\lambda$. É possível observar que conforme $\lambda$ aumenta a curva tende a uma reta em $y=1$. Neste exemplo $\lambda=500, \lambda=1000$, $\lambda=5000$ e $\lambda=10000$.

extinção de espécies em dinâmica de populações [4]. No entanto ela gera dificuldades conceituais, como o fato de não ser bijetora na região nula. O objetivo deste trabalho é justamente entender esse fenômeno e propor uma solução consistente.

Uma importante característica desta generalização é que ela é estritamente não negativa, uma vez que $\mathrm{e}_{\lambda}(t) \geq 0$. Outra característica peculiar da função exponencial tradicional válida também na generalizada é que $\mathrm{e}_{\lambda}(0)=1, \forall \lambda \in \mathbb{R}$. Outra propriedade válida para a generalização da função exponencial, similar ao que foi apontado para a função $\ln _{\lambda}(t)$ na Eq. (2.7), é que, dado $\alpha \neq 0$, tem-se:

$$
\left[\mathrm{e}_{\lambda}(t)\right]^{\alpha}=(1+\lambda t)^{\alpha / \lambda}=\left[1+\left(\frac{\lambda}{\alpha}\right) \alpha t\right]^{\alpha / \lambda}=\mathrm{e}_{\lambda / \alpha}(\alpha t) .
$$

Em particular, se $\alpha=-1$,

$$
\left[\mathrm{e}_{\lambda}(t)\right]^{-1}=\mathrm{e}_{-\lambda}(-t)
$$

que para $\lambda \rightarrow 0$, retorna a conhecida propriedade $\left[\mathrm{e}_{0}(t)\right]^{-1}=\mathrm{e}_{0}(-t)$.

Além destas características próprias de funções exponenciais presentes tam- 
bém na generalização, pode-se destacar algumas outras propriedades. Na álgebra tradicional, sabe-se que dados $a, b \in \mathbb{R}, \mathrm{e}^{a} \mathrm{e}^{b}=\mathrm{e}^{a+b}$. Na versão generalizada, não existe tal relação de forma direta, sendo necessário o uso dos operadores generalizados apresentados na Sec. 2.4. Desta forma,

$$
\mathrm{e}_{\lambda}(a) \cdot \mathrm{e}_{\lambda}(b)=\mathrm{e}_{\lambda}(a+b+\lambda a b)=\mathrm{e}_{\lambda}\left(a \oplus_{\lambda} b\right),
$$

em que $\oplus_{\lambda}$ é a soma generalizada apresentada na Eq. (2.23). Outra relação pode ser obtida mediante o uso do operador apresentado na Eq. (2.25),

$$
\mathrm{e}_{\lambda}(a+b)=\mathrm{e}_{\lambda}(a) \otimes_{\lambda} \mathrm{e}_{\lambda}(b)
$$

Nos dois casos, $\lambda=0$ retoma a validade da propriedade para a função tradicional.

Outras características próprias da função exponencial tradicional são observáveis na versão generalizada. Por exemplo, a derivada da função exponencial usual é a própria exponencial. É esperado, portanto, para as generalizações, uma expressão que envolva a própria função generalizada. E de fato:

$$
\frac{d}{d t} \mathrm{e}_{\lambda}(t)=\frac{(1+\lambda t)^{\frac{1}{\lambda}}}{1+\lambda t}=(1+\lambda t)^{\frac{1-\lambda}{\lambda}}=\left[\mathrm{e}_{\lambda}(t)\right]^{1-\lambda} .
$$

A exponencial generalizada é a solução da seguinte equação não linear:

$$
\frac{d y}{d t}=y^{1-\lambda}
$$

que é frequentemente encontrada em dinâmica de populações e cinética química [40].

Uma expressão mais geral pode ser calculada. Considerando a $n$-ézima derivada da função $e_{\lambda}(t)$ tem-se,

$$
\frac{d^{n}}{d t^{n}} \mathrm{e}_{\lambda}(t)=\left[\mathrm{e}_{\lambda}(t)\right]^{1-\lambda n} \prod_{j=0}^{n-1}(1-j \lambda) .
$$

Quando $\lambda=0$, retoma-se a propriedade de que qualquer derivada da função exponencial tradicional é a própria função.

Quanto a integração, tem-se, para a função tradicional,

$$
\int \mathrm{e}^{t} d t=\mathrm{e}^{t}+C
$$

com $C$ uma constante de integração. Já para a função generalizada, a expressão obtida mediante integração pode ser apresentada como

$$
\int \mathrm{e}_{\lambda}(t) d t=\left(\frac{1+\lambda t}{1+\lambda}\right) \mathrm{e}_{\lambda}(t)+C .
$$


Também neste caso, se $\lambda=0$, retoma-se que a integral da exponencial convencional é a própria função acrescido de uma constante.

A exemplo do que foi feito com a função $\ln _{\lambda}(t)$, pode-se considerar o parâmetro $\lambda$ como variável lenta. Desta forma, abre-se a possibilidade de encontrar a derivada da função $e_{\lambda}(t)$ em relação a $\lambda$, que neste caso é dada por:

$$
\frac{d}{d \lambda} \mathrm{e}_{\lambda}(t)=\frac{\mathrm{e}_{\lambda}(t)}{\lambda}\left\{\frac{t}{1+\lambda t}-\ln \left[\mathrm{e}_{\lambda}(t)\right]\right\}
$$

mantendo a característica de que a derivada da exponencial continua resultando em um produto que envolve a própria função.

Mesmo em se tratando das derivadas de ordem superior, é possível escrever uma fórmula de recorrência que envolve o produto da exponencial generalizada. Esta expressão é dada por

$$
\begin{aligned}
\frac{d^{n}}{d \lambda^{n}} \mathrm{e}_{\lambda}(t) & =\mathrm{e}_{\lambda}(t)\left\{(-1)^{n} n !\left[\frac{\ln \left[\mathrm{e}_{\lambda}(t)\right]}{\lambda^{n}}-\sum_{j=1}^{n} \frac{\lambda^{-j}}{(n+1-j)}\left(\frac{t}{1+\lambda t}\right)^{n+1-j}\right]+\right. \\
& \left.-\sum_{i=1}^{n-1}\left(\begin{array}{c}
n-1 \\
i-1
\end{array}\right)\left(\frac{d^{n-i}}{d \lambda^{n-i}}\left[\mathrm{e}_{\lambda}(t)\right]^{-1}\right)\left(\frac{d^{i}}{d \lambda^{i}} \mathrm{e}_{\lambda}(t)\right)\right\}
\end{aligned}
$$

Apesar da complexidade da expressão, verifica-se que ela é um produto da exponencial generalizada com somas de derivadas desta mesma função, algo também esperado para funções exponenciais. Os cálculos relativos a obtenção desta expressão podem ser verificados no Apêndice 1.

Considere a validade da seguinte equação em termos da função exponencial tradicional:

$$
\left(\mathrm{e}^{t}\right)^{-1} \frac{d}{d t} \mathrm{e}^{t}+\mathrm{e}^{t} \frac{d}{d t}\left(\mathrm{e}^{t}\right)^{-1}=0 .
$$

O mais impressionante é que esta mesma relação também é valida no contexto generalizado. Quando utiliza-se as derivadas em relação a $t$ segue que

$$
\left[\mathrm{e}_{\lambda}(t)\right]^{-1} \frac{d}{d t} \mathrm{e}_{\lambda}(t)+\mathrm{e}_{\lambda}(t) \frac{d}{d t}\left[\mathrm{e}_{\lambda}(t)\right]^{-1}=0, \forall \lambda, t \in \mathbb{R} .
$$

Quando apresenta-se a mesma expressão com as derivadas em relação à $\lambda$ observa-se que

$$
\left[\mathrm{e}_{\lambda}(t)\right]^{-1} \frac{d}{d \lambda} \mathrm{e}_{\lambda}(t)+\mathrm{e}_{\lambda}(t) \frac{d}{d \lambda}\left[\mathrm{e}_{\lambda}(t)\right]^{-1}=0, \forall \lambda, t \in \mathbb{R},
$$

mantendo a validade e, mais do que isso, indicando que a generalização da função exponencial parece consistente. 
Neste ponto, faz-se necessária a seguinte análise: na Eq. (2.47), percebe-se que o termo exponencial tanto pode ser escrito como $\left(\mathrm{e}^{t}\right)^{-1}$ quanto, de forma mais direta, $\mathrm{e}^{-t}$. Esta alteração não produz qualquer efeito sobre o resultado final já que é próprio da função exponencial $\left(\mathrm{e}^{t}\right)^{\omega}=\left(\mathrm{e}^{\omega}\right)^{t}=\mathrm{e}^{\omega t}, \forall \omega, t$. Já nas formas generalizadas, isso não acontece. Substituir $\left[\mathrm{e}_{\lambda}(t)\right]^{-1}$ por $\mathrm{e}_{\lambda}(-t)$ na Eq. (2.48) gera a seguinte expressão:

$$
\mathrm{e}_{\lambda}(-t) \frac{d}{d t} \mathrm{e}_{\lambda}(t)+\mathrm{e}_{\lambda}(t) \frac{d}{d t} \mathrm{e}_{\lambda}(-t)=-2 \lambda t\left[1-\lambda^{2} t^{2}\right]^{\frac{1-\lambda}{\lambda}},
$$

que só se anula no limite de $\lambda \rightarrow 0$. Na Eq. (2.49), a mesma substituição produz a seguinte expressão:

$$
\begin{aligned}
\mathrm{e}_{\lambda}(-t) & \frac{d}{d \lambda} \mathrm{e}_{\lambda}(t)+\mathrm{e}_{\lambda}(t) \frac{d}{d \lambda} \mathrm{e}_{\lambda}(-t)= \\
& =\frac{\left(1-\lambda^{2} t^{2}\right)^{\frac{1-\lambda}{\lambda}}}{\lambda^{2}}\left[\left(\lambda^{2} t^{2}-1\right) \ln \left(1-\lambda^{2} t^{2}\right)-2 \lambda^{2} t^{2}\right] .
\end{aligned}
$$

No caso da Eq. (2.51) não faz muito sentido calcular o limite quando $\lambda \rightarrow 0$, já que a equação diferencial tem derivada em relação a $\lambda$. Faz-se, então, o limite de $t \rightarrow 0$ na Eq. (2.51), que produz o valor nulo.

Nesta mesma linha de raciocínio, poder-se-ia tentar substituir, na Eq. (2.49), $\left[\mathrm{e}_{\lambda}(t)\right]^{-1}$ por $\mathrm{e}_{-\lambda}(t)$. Esta ação traria como resultado

$$
\mathrm{e}_{\lambda}(t) \frac{d}{d \lambda} \mathrm{e}_{-\lambda}(t)+\mathrm{e}_{-\lambda}(t) \frac{d}{d \lambda} \mathrm{e}_{\lambda}(t)=\frac{\mathrm{e}_{\lambda}(t)}{\lambda \mathrm{e}_{\lambda}(-t)}\left\{\frac{2 t}{1-\lambda^{2} t^{2}}+\ln \left[\frac{\mathrm{e}_{\lambda}(-t)}{\mathrm{e}_{\lambda}(t)}\right]\right\},
$$

o qual se anula somente quando $\lambda \rightarrow 0$. Usando a Eq. (2.37), pode-se reescrever a Eq. (2.48) como,

$$
\left[\mathrm{e}_{-\lambda}(-t)\right] \frac{d}{d t} \mathrm{e}_{\lambda}(t)+\mathrm{e}_{\lambda}(t) \frac{d}{d t}\left[\mathrm{e}_{-\lambda}(-t)\right]=0, \forall \lambda, t \in \mathbb{R} .
$$

A Eq. (2.49), também pode ser reescrita utilizando o mesmo argumento.

Essas observações nos levam a uma discussão mais ampla: como devem ser generalizadas expressões que, na versão original, contenham o núcleo $\left(\mathrm{e}^{t}\right)^{\omega}=\mathrm{e}^{\omega t}$ ? Seria $\mathrm{e}_{\lambda}(\omega t)$ ? Ou então $\left[\mathrm{e}_{\lambda}(t)\right]^{\omega}$ ? Este questionamento ganha um caráter fundamental na análise complexa. Por exemplo, qual expressão seria mais apropriada para generalizar $e^{i \omega t}: \mathrm{e}_{\lambda}(i \omega t),\left[\mathrm{e}_{\lambda}(i t)\right]^{\omega}$ ou $\left[\mathrm{e}_{\lambda}(t)\right]^{i \omega}$ ? A Fig. 2.6 representa este questionamento.

Apesar da incerteza de como generalizar a parte imaginária de uma variável complexa para variáveis reais, a generalização da função exponencial cumpre bem 


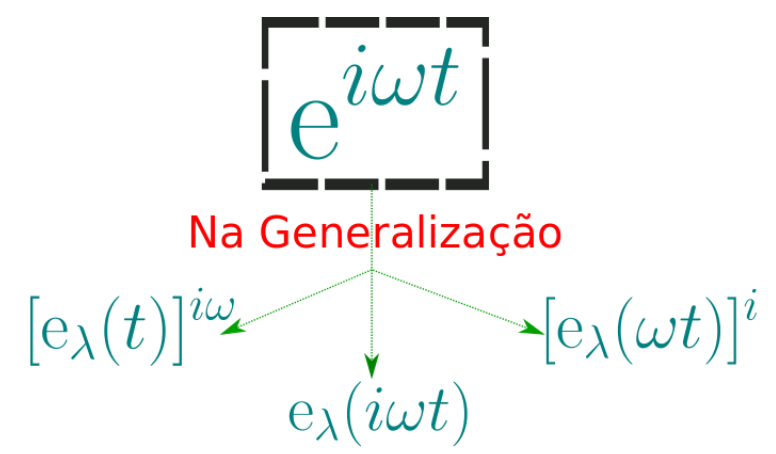

Figura 2.6: Possibilidades de interpretação da função $\mathrm{e}^{i \omega t}$ após a generalização. Note que $\mathrm{e}^{i \omega t}=$ $\left(\mathrm{e}^{\omega t}\right)^{i}=\left(\mathrm{e}^{t}\right)^{i \omega}$. Contudo, $\mathrm{e}_{\lambda}(i \omega t) \neq\left[\mathrm{e}_{\lambda}(\omega t)\right]^{i} \neq\left[\mathrm{e}_{\lambda}(t)\right]^{i \omega}$. Nota-se que, $\mathrm{e}_{\lambda}(i \omega t)$ não é uma expressão analítica. Por outro lado $\left[\mathrm{e}_{\lambda}(\omega t)\right]^{i}$ não favorece generalização de outras estruturas, como por exemplo, a transformada de Fourier.

seu papel, já que absorve propriedades e características próprias da versão tradicional. Desta forma, seria desejável que, também no plano complexo, a generalização mantivesse características próprias da função original, como por exemplo, a analiticidade. Isso nos leva ao descarte da expressão $e_{\lambda}(i \omega t)$, na Fig. 2.6. Na sequência, apresentamos nossa generalização da função exponencial cuja parte imaginária se mostra útil em transformações de Laplace e Fourier e, desta forma, acaba descartando também a expressão $\left[\mathrm{e}_{\lambda}(\omega t)\right]^{i}$ no questionamento apresentado na Fig. 2.6.

\subsection{A exponencial generalizada no plano complexo}

A Sec. 2.5 apresentou uma generalização para a função exponencial muito bem definida e que cumpre seu papel desde que se trabalhe com variáveis reais. Quando o universo de trabalho está nos números complexos, se faz necessário uma expansão dessa definição. Alguns autores fazem uma transição direta e até sutil, substituindo $t \in \mathbb{R}$ por $z \in \mathbb{C}$, sem indicar possíveis implicações. Percebe-se tal ação nas referências $[18,31,41,42]$. Contudo, tal medida não garante analiticidade da função em todo plano, divergindo do que ocorre com a função exponencial tradicional.

Uma função $f$ é analítica em um conjunto $D \subset \mathbb{C}$, se for diferenciável em cada ponto $z_{0} \in D$. Além disso, as funções analíticas (ou "holomorfas") possuem uma série de propriedades interessantes, como por exemplo, ter o Laplaciano nulo em todo domínio $D$. Outra vantagem é que em cálculos como, por exemplo, de integrais não estão sujeitos a análise sobre ramificações e singularidades [43]. 
Nosso objetivo, portanto, é ampliar o escopo da função exponencial generalizada, abrindo possibilidades de considerar também nas variáveis complexas uma função analítica, levando em conta as diversas possibilidades mostradas na Fig. 2.6. Para tanto, pode-se reescrever a Eq. (2.33) utilizando a norma (valor absoluto) e, desta forma,

$$
\mathrm{e}_{\lambda}(t)=\left\{\begin{array}{rc}
\lim _{\lambda^{\prime} \rightarrow \lambda}\left|1+\lambda^{\prime} t\right|^{1 / \lambda^{\prime}} & , \text { se } 1+\lambda^{\prime} t \geq 0 \\
0 & , \text { caso contrário } .
\end{array},\right.
$$

que não altera a definição apresentada na Eq.(2.33). A Utilização da função Heaviside, ou simplesmente "função degrau", definida por

$$
\theta(x)=\left\{\begin{array}{ll}
1 & , \text { se } x \geq 0 \\
0 & , \text { se } x<0
\end{array},\right.
$$

ajuda na simplificação da Eq. (2.54), como já observado na Ref. [41]. Desta forma, uma definição mais limpa para a generalização da função exponencial é dada por

$$
\mathrm{e}_{\lambda}(t)=\lim _{\lambda^{\prime} \rightarrow \lambda}\left|1+\lambda^{\prime} t\right|^{\frac{1}{\lambda^{\prime}}} \theta(1+\lambda t) .
$$

Nossa proposta é aproveitar a simplicidade e consistência da definição real, apresentada nas Eqs. (2.54) e (2.56), unida à uma expressão analítica no plano complexo. Desta forma, de maneira direta, apresentamos uma nova extensão da função exponencial generalizada para argumentos complexos:

$$
\mathrm{e}_{\lambda}(t)=\lim _{\lambda^{\prime} \rightarrow \lambda}\left|1+\lambda^{\prime} t\right|^{1 / \lambda^{\prime}}\left\{\begin{array}{cl}
1 & , \text { se } 1+\lambda^{\prime} t>0 \\
(-1)^{1 / \lambda^{\prime}} & , \text { se } 1+\lambda^{\prime} t \leq 0 .
\end{array}\right.
$$

A exemplo do que foi feito para se obter a Eq. (2.56), utilizamos a função degrau para simplificar nossa definição, de modo que

$$
\mathrm{e}_{\lambda}(t)=\lim _{\lambda^{\prime} \rightarrow \lambda}\left|1+\lambda^{\prime} t\right|^{\frac{1}{\lambda^{\prime}}} \mathrm{e}^{i \frac{\pi}{\lambda^{\prime}}\left[1-\theta\left(1+\lambda^{\prime} t\right)\right]} .
$$

Observe que, se $1+\lambda t \geq 0$, mantém-se a definição para a função com variáveis reais, caso contrário, abre-se as portas para uma nova definição no plano complexo com uma função analítica. O fator $|1+\lambda t|^{1 / \lambda}$ é a amplitude e $(\pi / \lambda)[1-\theta(1+\lambda t)]$ a fase da função exponencial generalizada no plano complexo.

O objetivo, aqui, é escrever a função exponencial generalizada no plano complexo como sendo uma função exponencial convencional com variáveis complexas. 
Para tanto faz-se:

$$
\mathrm{e}^{z}=\mathrm{e}^{\ln |1+\lambda t|^{1 / \lambda}} \mathrm{e}^{i \frac{\pi}{\lambda}[1-\theta(1+\lambda t)]},
$$

com $z \in \mathbb{C}$ e de tal forma que,

$$
z=\ln |1+\lambda t|^{\frac{1}{\lambda}}+i \frac{\pi}{\lambda}[1-\theta(1+\lambda t)] .
$$

Dessa forma, é possível escrever um número complexo com as partes real e imaginária

$$
\begin{aligned}
& \operatorname{Re}(z)=\ln |1+\lambda t|^{\frac{1}{\lambda}}=a \\
& \operatorname{Im}(z)=\frac{\pi}{\lambda}[1-\theta(1+\lambda t)]=b .
\end{aligned}
$$

Em outras palavras,

$$
\mathrm{e}^{z}=\mathrm{e}^{a}\left(\mathrm{e}^{b}\right)^{i}
$$

que, assim como a função exponencial tradicional, é analítica em $\mathbb{C}$. A Fig.(2.7) apresenta o comportamento das partes real e imaginária de $\mathrm{e}_{\lambda}(t)$. Nela, é possível verificar que para $\lambda=3$, por exemplo, a função é real para $t \geq-1 / 3$, ou complexa do tipo $z=k+m i,(k, m) \neq(0,0)$, caso contrário. Nesse exemplo, não existem regiões que retornam um imaginário puro. Por outro lado, para $\lambda=2$ é possível verificar que ou tem-se como imagem da função um imaginário puro (se $t<-1 / 2$ ) ou um número real (se $t \geq-1 / 2$ ). Para $\lambda=0$, a saída é uma função real.

Em suma, a nossa generalização da função exponencial para variáveis complexas consiste em manter a generalização para variáveis reais e generalizar a parte imaginária na potência $i$, de tal forma que

$$
\mathrm{e}^{a+b i} \rightarrow \mathrm{e}_{\lambda}(a)\left[\mathrm{e}_{\lambda}(b)\right]^{i}
$$

Essa implicação será de fundamental importância na definição de novas generalizações, como será apresentado mais adiante.

Voltando à discussão de generalizações consistentes, é possível afirmar que esta expansão da definição de $e_{\lambda}(t)$ para o plano complexo acontece de forma muito natural, sem a necessidade de "remediar" possíveis problemas que garantam sua continuidade ou analiticidade. Com esta definição, mantém-se as principais características e propriedades da função exponencial quando estudada em $\mathbb{C}$, inclusive a analiticidade. Doravante, em nosso trabalho, ao utilizar a expressão $e_{\lambda}(t)$, estaremos nos referindo à nossa definição da função exponencial generalizada, de acordo com 

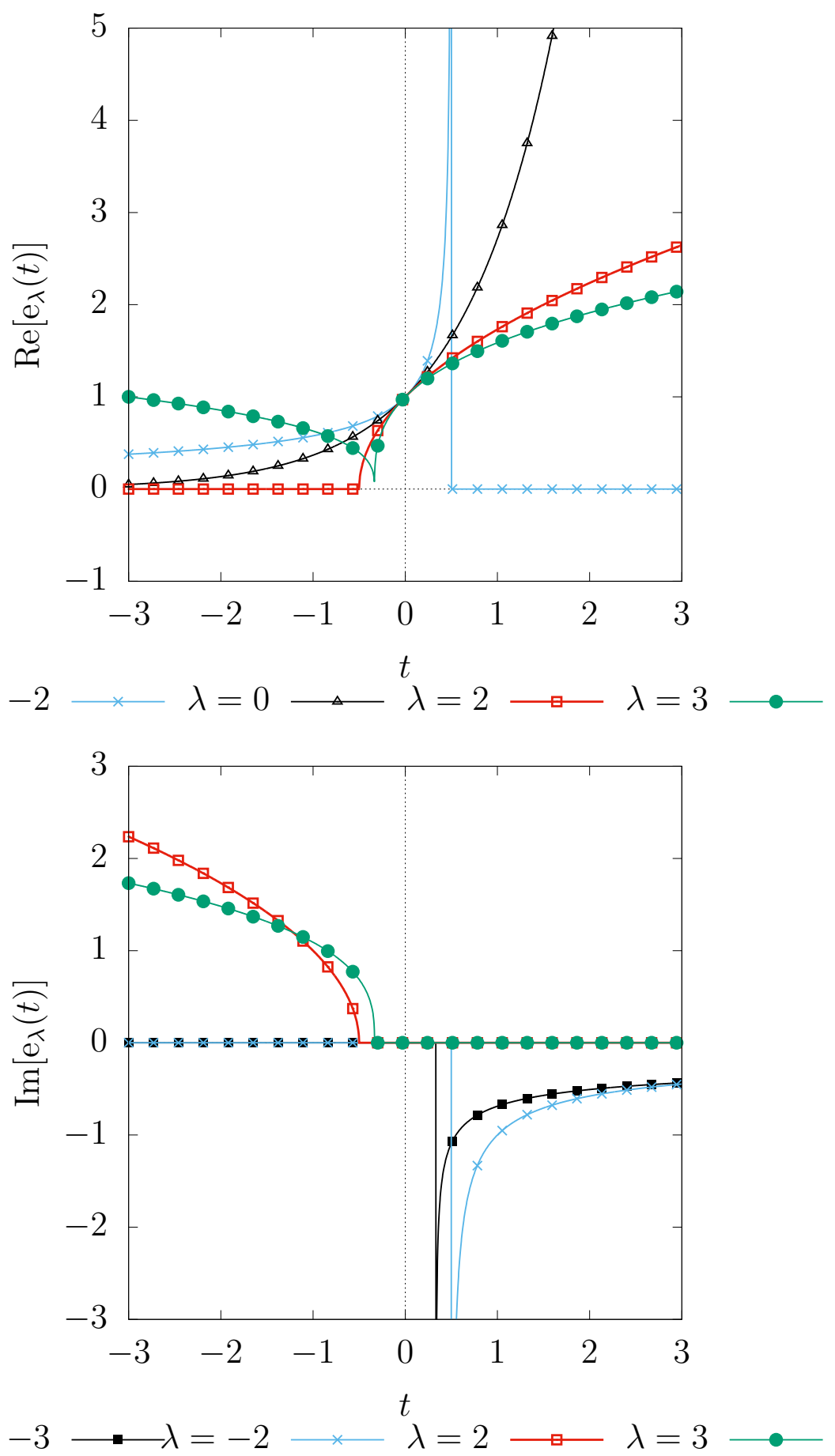

Figura 2.7: Comportamento de algumas curvas da função $e_{\lambda}(t)$ com saída em $\mathbb{C}$. a) Parte real da função $\mathrm{e}_{\lambda}(t)$ para os valores de $\lambda=-2, \lambda=0, \lambda=2, \lambda=3$. b) Parte imaginária da função $\mathrm{e}_{\lambda}(t)$ para os valores de $\lambda=-3, \lambda=-2, \lambda=2, \lambda=3$. Pode-se perceber que, nestes exemplos, a função tem como imagem números reais ou números complexos do tipo $z=a+b i$. Nunca imaginários puros. 
a Eq. (2.59), ou seja,

$$
\mathrm{e}_{\lambda}(t)=\lim _{\lambda^{\prime} \rightarrow \lambda}\left|1+\lambda^{\prime} t\right|^{\frac{1}{\lambda^{\prime}}} \mathrm{e}^{i \frac{\pi}{\lambda^{\prime}}\left[1-\theta\left(1+\lambda^{\prime} t\right)\right]} .
$$

Nesta primeira parte do trabalho, foram revisados conceitos importantes relativos à operadores e funções generalizadas. Reviu-se ainda definições relativas à função logaritmo generalizada $\ln _{\lambda}(t)$ bem como de sua inversa, a função exponencial generalizada $e_{\lambda}(t)$. É possível observar que a generalização da função logaritmo apresentada possui muitas características e propriedades da tradicional. Algumas aparecem de forma natural, com as operações tradicionais, outras nem tanto. Contudo, nenhuma propriedade importante ou característica própria de funções logaritmo é perdida nesta generalização, apontando que a mesma está bem "contextualizada". O mesmo ocorre quanto a função exponencial generalizada, principalmente após nossa proposta de expansão para o plano complexo: ambas mantém suas características e propriedades. Quanto aos operadores binários generalizados, inserimos um olhar matemático sobre a formação de uma estrutura de "anel generalizado". Tais temas foram discutidos mediante a reflexão quanto ao que são generalizações consistentes e têm importância fundamental nos próximos capítulos. 


\section{3}

\section{Transformações de Box-Cox}

Na vida, não existe nada a temer, mas a entender.

- Marie Curie

O estudo estatístico de dados é necessário para a compreensão de fenômenos. Porém, nem sempre se encontra facilmente um estimador pertinente em todos os casos e, desta forma, disponibilizar ferramentas que tornem este trabalho menos oneroso é de suma importância. Uma das primeiras medidas a se tomar é o estudo referente a distribuição dos dados em questão, na tentativa de transformar os dados de modo que a nova variável siga uma distribuição Gaussiana. Nesta perspectiva, todo arsenal de métodos de estatística paramétrica pode ser seguramente aplicados.

Box e Cox [19] apresentaram na década de 1960 uma interessante transformação capaz de levar dados que seguem distribuições com cauda pesada à Gaussianas. Conforme mencionado no Cap. 2, a transformação de Box-Cox pode ser descrita pela função logaritmo generalizado $\ln _{\lambda}(t)$. Seguindo as cronologias de Sakia [20] e Daimon [21], apresentamos aqui uma breve revisão de algumas transformações inspiradas na transformação de Box-Cox, relacionando-as com esta função. A novidade aqui é expressar essas transformações usando as funções exponencial e logaritmo generalizadas, facilitando assim a compreensão de seu uso e as implementações.

\subsection{Contexto histórico}

Transformação de dados para análise estatística é um tema discutido desde meados do séc. XIX. De acordo com Aitchison [44], as primeiras transformações consistentes foram pensadas por McAlister, em 1879, com o objetivo de simplificar 
a análise de dados empíricos. Depois disso, muitos estudiosos se dedicaram a esta tarefa que, até a década de 1950, estava restrita a transformações logarítmicas e de raiz. Em 1952, Ascombe e Tukey[45] apresentam a primeira família de transformações. Essa ideia foi melhor desenvolvida em publicações posteriores [46, 47] até que, em 1964, Box e Cox [19] apresentam sua família de transformações, que despertaram grande interesse por conta da eficiência, facilidade de uso e por unificar as transformações logarítmicas e de raízes.

O Professor George Edward Pelham Box nasceu em 18 de outubro de 1919 em Gravesend, Kent na Inglaterra. Iniciou sua trajetória acadêmica na área de Química mas não concluiu pois foi convocado para o serviço militar, onde realizou algumas experiências para o exército britânico. Este trabalho consistia na exposição de pequenos animais a um gás venenoso, o que o levou ao estudo da estatística dos resultados obtidos. Após a guerra, se matriculou e obteve o diploma de bacharel em matemática e estatística na University College London. Já em 1953, finalizou o doutorado sob orientação de Egon Pearson na Universidade de Londres. Trabalhou como estatístico na Indústria Química Imperial e passou por grandes universidades, tais como a Universidade Estadual da Carolina do Norte e a Universidade de Princeton, onde atuou como diretor de um grupo de pesquisa. Em 1960, mudou-se para a universidade de Wisconsin-Madison para criar o departamento de Estatística, onde recebeu diversas honrarias e desenvolveu um trabalho exemplar até sua aposentadoria em 1992. Ele foi autor da célebre frase "Todos os modelos estão errados" e que é frequentemente aumentada para "Todos os modelos estão errados, mas alguns são úteis". Foi casado com Joan Fisher, filha de Sir Ronald Fisher e faleceu aos 93 anos no dia 28 de março de 2013 em Wisconsin [48].

Sir David Roxbee Cox nasceu em 15 de julho de 1924 em Birmingham, na Inglaterra, onde seu pai era co-proprietário de uma loja de jóias. Iniciou seus estudos na escola de gramática Handsworth. Estudou matemática no colégio St. John, instituição ligada à Universidade de Cambridge. Concluiu seu doutorado na Universidade de Leeds em 1949, onde foi orientado por Henry Daniels e Bernard Welch. Passou por várias instituições, dentre elas Royal Aircraft Establishment, Universidade de Cambridge e Imperial College London, onde mais tarde se tornou chefe do departamento de Matemática. Em 1988, tornou-se diretor do Nuffield College 
e membro do Departamento de Estatística da Universidade de Oxford. Se aposentou formalmente desses cargos em 1994. Possui publicações de peso e alguns procedimentos até hoje utilizados, como por exemplo o processo de Cox. Segundo Memória [49], Cox tem diversos prêmios por seu desempenho acadêmico, dentre os quais destaca-se a Medalha Copley (2010).

Alguns historiadores afirmam que a família de transformações apresentada por Box e Cox surgiu quase que por uma brincadeira. Enquanto Cox visitava Box em Wisconsin, decidiram que deveriam escrever algo juntos pela semelhança de seus nomes e pelo fato de ambos serem britânicos [50]. Desta forma, trabalharam juntos no brilhante artigo "An analysis of transformation" [19] de 1964.

O estudo desenvolvido por estes dois cientistas se tornou um marco na história da Estatística, já que a transformação de dados muitas vezes é o único recurso para a análise de distribuições inicialmente apresentadas de formas complicadas. Assim, uma "normalização" eficaz de dados facilita, aprimora e torna mais preciso a análise e, como consequência, a tomada de decisão em pesquisas nas diversas áreas do conhecimento. Na sequência, apresentamos um histórico das principais transformações do tipo Box-Cox. Nele tomamos a liberdade de escrever tais transformações utilizando a função logaritmo generalizada, o que gerou simplicidade de notação e maior facilidade para visualizar algumas características, como por exemplo a simetria existente na versão da transformação apresentada por Yeo e Johnson [22].

\subsection{Transformações do tipo Box-Cox}

A busca de Ascombe, Tukey, Box e Cox e tantos outros por transformações de dados continua sendo de suma importância para ações mediante informações empíricas. É desejável que os dados coletados estejam distribuídos de maneira a facilitar os estudos e observações. É mais desejável ainda que a disposição destes dados seja de acordo com uma curva gaussiana - função densidade de probabilidade normal. Essa distribuição é simétrica, centrada na média $(\mu)$ e 99,7\% dos dados estão localizados no intervalo $[\mu-2 \sigma, \mu+2 \sigma]$, em que $\sigma$ é o desvio padrão. Além disso, por ser o limite de um grande número de distribuições de probabilidade (teorema do limite central), a distribuição normal permite estudar probabilisticamente a média das variáveis independentes de uma amostra aleatória e modelar, desta forma, vários 
estudos científicos como, por exemplo, erros e testes [51].

Tukey, em 1957, apresentou a primeira família de transformações consistente. Na verdade, ele conseguiu sintetizar em uma única expressão tudo o que já era utilizado com certa eficiência - transformações logarítmicas e de raiz - e ainda ampliou. De fato, ele introduziu uma família de transformações de potência indexadas por um parâmetro $\lambda$,

$$
y_{i}^{(\lambda)}=\left\{\begin{array}{rl}
y_{i}^{\lambda} & , \text { se } \lambda \neq 0 \\
\ln \left(y_{i}\right) & , \text { se } \lambda=0
\end{array},\right.
$$

com $y_{i}>0$. Por exemplo, o caso mais simples $(\lambda=0)$ é pertinente para o estudo de processos multiplicativos pois, nesses casos, $\ln \left(y_{i}\right)$ segue uma função densidade de probabilidade normal e $y_{i}$ uma lognormal. No entanto, essas transformações não levam em conta a descontinuidade quando $\lambda \rightarrow 0$ e nem tão pouco apresentam alternativas caso existam valores $y_{i} \leq 0$.

Box e Cox reobtiveram uma transformação semelhante a Eq. (3.1). Eles utilizaram o conceito de máxima verossimilhança e propuseram a seguinte transformação:

$$
y_{i}^{(\lambda)}=\left\{\begin{array}{cc}
\frac{y_{i}^{\lambda}-1}{\lambda} & , \text { se } \lambda \neq 0 \\
\ln \left(y_{i}\right) & , \text { se } \lambda=0 .
\end{array}\right.
$$

que, conforme apresentado no Cap. 2, é exatamente a definição de logaritmo generalizado. Assim

$$
y_{i}^{(\lambda)}=\ln _{\lambda}\left(y_{i}\right), y_{i}>0
$$

Utilizar $\ln _{\lambda}(y)$ na apresentação da transformação de Box-Cox, além de apresentar continuidade analítica em $\lambda=0$, uma vez que $\lim _{\lambda^{\prime} \rightarrow 0}\left(y^{\lambda^{\prime}}-1\right) / \lambda^{\prime}=\ln (y)$, gera facilidade na notação em possíveis cálculos, haja visto que existem muitas propriedades relativas à esta generalização. Desta forma, conforme já apontado, tomamos a liberdade de apresentar as demais transformações escritas em função do logaritmo generalizado, mesmo sendo algumas definições anteriores à esta generalização.

Em uma rápida análise sobre a proposta de Box e Cox, pode-se verificar que não foi uma tentativa inocente para chegar à normalização dos dados. Levando-se em conta que, até aquele momento, os melhores resultados de transformação eram obtidos por meio de transformações logarítmicas, é bastante lógico supor que, em uma "generalização" deste mecanismo sobre um determinado parâmetro $\lambda$ deverá 
existir um $\lambda_{0}$ que melhor aproxime os dados empíricos de uma função densidade de probabilidade normal. É claro que a expressão "logaritmo generalizado" só viria surgir posteriormente, mas o conceito já estava sendo aplicado.

A ideia de logaritmo está tão presente na transformação de Box-Cox que os autores identificam um possível problema e já apontam uma solução. A primeira transformação de Box-Cox não leva em conta valores negativos de $y_{i}$ e, como solução, toma-se um valor $\lambda_{2}>\min \left(y_{i}\right)$ de forma que, $\lambda_{2}-y_{i}>0, \forall i$, tem-se

$$
y_{i}^{(\lambda)}=\ln _{\lambda}\left(y_{i}+\lambda_{2}\right) .
$$

Essa transformação é conhecida como Box-Cox com "translação", aja visto que desloca os valores de todos os dados empíricos para $\mathbb{R}^{+}$.

Posteriormente, em 1976, Manly [52] apresentou uma nova alternativa para lidar com valores negativos: utilizar a função exponencial. Assim,

$$
y_{i}^{(\lambda)}=\ln _{\lambda}\left(e^{y_{i}}\right)
$$

Tal transformação apresenta excelentes resultados quando aplicada à distribuições unimodais, porém, o mesmo não pode ser dito em caso de outras distribuições de dados.

Já em 1980, John e Draper [53] propuseram a chamada transformação modular. Ela também lida bem com valores negativos, pois utiliza, conforme sugerido pelo nome, o valor absoluto do dado observado:

$$
y_{i}^{(\lambda)}=\operatorname{Sign}\left(y_{i}\right)\left[\ln _{\lambda}\left(\left|y_{i}\right|+1\right)\right]
$$

em que

$$
\operatorname{Sign}\left(y_{i}\right)=\left\{\begin{array}{r}
1 \quad, \text { se } y_{i} \geq 0 \\
-1 \quad, \text { se } y_{i}<0
\end{array} .\right.
$$

Bickel e Doksum [54] também utilizam o valor absoluto dos dados empíricos para fugir de possíveis descontinuidades na transformação. A proposta apresentada por eles também pode ser escrita através do logaritmo generalizado como,

$$
y_{i}^{(\lambda)}=\ln _{\lambda}\left\{\left|y_{i}\right|\left[\operatorname{Sign}\left(y_{i}\right)\right]^{\frac{1}{\lambda}}\right\} .
$$

Esta expressão é a mais simples possível para esta transformação, porém acaba negligenciando, em alguns casos, a questão da continuidade, já que para $\lambda=2$, por 
exemplo, os valores de $y_{i}$ 's negativos incorrerão em uma abordagem com números complexos. Uma alternativa de apresentação para essa transformação em termos de $\ln _{\lambda}(t)$, é a seguinte:

$$
y_{i}^{(\lambda)}=\operatorname{Sign}\left(y_{i}\right) \ln _{\lambda}\left(\left|y_{i}\right|\right)-\frac{2}{\lambda} \theta\left(y_{i}\right),
$$

em que $\theta$ é a função Heaviside,

$$
\theta(x)=\left\{\begin{array}{ll}
1 & , \text { se } x \geq 0 \\
0 & , \text { se } x<0
\end{array} .\right.
$$

Ainda buscando uma solução para o problema de números negativos nas transformações de Box-Cox, Yeo e Johnson [22] defenderam a seguinte transformação:

$$
y_{i}^{(\lambda)}=\left\{\begin{array}{ll}
+\ln _{(\lambda)}\left(1+y_{i}\right) & , \text { se } y_{i} \geq 0 \\
-\ln _{(2-\lambda)}\left(1-y_{i}\right) & , \text { se } y_{i}<0
\end{array} .\right.
$$

Essa transformação foi pensada no sentido de encontrar $\lambda$ que minimize a distância de Kullback-Leibler ${ }^{1}$ entre a função densidade de probabilidade original e a normal. Além disso, pode-se considerar esta transformação como a mais "natural", já que utiliza a simetria de rotação em torno da bissetriz das funções que geram a função logaritmo generalizado.

Todas essas transformações funcionam muito bem (ou muito mal) dependendo do valor atribuído a $\lambda$. Depois do surgimento dessas famílias de funções que buscam organizar os dados de acordo com uma distribuição normal, o maior desafio é encontrar um valor para este parâmetro de tal forma que o erro entre a distribuição de dados obtidas e uma Gaussiana seja mínimo. Montegory e Peck [55] propõem a utilização de um método de regressão linear via mínimos quadrados ordinários (MQO) para auxiliar nesta tarefa. Para que isso seja plausível quanto à escalas, é necessário normalizar os dados pela sua média geométrica $g$. Desta forma, a transformação de Box-Cox normalizada é dada por:

$$
y_{i}^{(\lambda)}=\frac{1}{g^{\lambda-1}} \ln _{\lambda}\left(y_{i}\right)
$$

À seguir, apresentamos duas maneiras de se obter $\lambda$ via o princípio da máxima verossimilhança.

\footnotetext{
${ }^{1}$ também conhecida como entropia relativa
} 


\subsection{Estimativa do parâmetro $\lambda$}

Até aqui, apresentamos a evolução da transformação de Box-Cox durante algumas décadas e as escrevemos utilizando a função logaritmo generalizado. Em todas estas famílias de transformações existe um valor a ser obtido: o parâmetro $\lambda$. Este número, calculado de forma adequada, aproxima a distribuição original à gaussiana e pode ser encontrado utilizando o princípio de máxima verossimilhança [56].

Seja $\lambda$ um parâmetro desconhecido, $Y$ uma variável aleatória e $y=$ $y_{1}, y_{2}, \cdots, y_{n}$ uma amostra simples de tamanho $n$ de $Y$. Seja ainda $y^{(\lambda)}=$ $y_{1}^{(\lambda)}, y_{2}^{(\lambda)}, \cdots, y_{n}^{(\lambda)}$ os valores transformados de $y_{i}$ sob o parâmetro $\lambda$ de tal forma que $y^{(\lambda)} \sim N\left(\mu, \sigma^{2}\right)$. Desta forma, pode-se escrever

$$
\vec{y}^{(\lambda)}=\left(y_{1}^{(\lambda)}, y_{2}^{(\lambda)}, y_{3}^{(\lambda)}, \cdots, y_{n}^{(\lambda)}\right)=X \vec{\kappa}+\vec{\varepsilon},
$$

em que $X$ é uma matriz de valores constantes, $\kappa$ é um vetor de parâmetros desconhecidos associado aos valores transformados e $\vec{\varepsilon}$ é um vetor de erros aleatórios com média $\mu_{\varepsilon}=0$ e $\sigma_{\varepsilon}^{2}$ suficientemente pequeno. Sob estas condições, pode-se escrever $f\left(y^{(\lambda)}\right)$, a função densidade de $y^{(\lambda)}$, como

$$
f\left(y^{(\lambda)}\right)=\frac{1}{\left(\sqrt{2 \pi} \sigma_{\varepsilon}\right)^{n}} \exp \left[-\frac{1}{2 \sigma_{\varepsilon}^{2}}\left(\vec{y}^{(\lambda)}-X \vec{\kappa}\right)^{\prime} \cdot\left(\vec{y}^{(\lambda)}-X \vec{\kappa}\right)\right] .
$$

Seja $J(\lambda, y)$ o jacobiano da transformação de $y$ para $y^{(\lambda)}$ que, no caso de Box-Cox, é escrito como

$$
J(\lambda, y)=\prod_{i=1}^{n} y_{i}^{\lambda-1}
$$

Desta forma e nestas condições, a função de máxima verossimilhança é dada

$$
\begin{aligned}
& L\left(\lambda, y_{1}, y_{2}, \cdots, y_{n}\right)= \\
& \quad=f(y)=\frac{1}{\left(\sqrt{2 \pi} \sigma_{\varepsilon}\right)^{n}} \exp \left[-\frac{1}{2 \sigma_{\varepsilon}^{2}}\left(\vec{y}^{(\lambda)}-X \vec{\kappa}\right)^{\prime} \cdot\left(\vec{y}^{(\lambda)}-X \vec{\kappa}\right)\right] J(\lambda, y) .
\end{aligned}
$$

Maximizando $L$ em relação a $\lambda$, encontra-se o valor ótimo para este parâmetro, ou seja, o valor que faz com que $L$ melhor se aproxime da função $f$ apresentada. Por facilidade, em alguns casos pode-se utilizar a log-verossimilhança, $\ell=\ln (L)$, ao invés de $L$. Neste caso,

$$
\ell \equiv \ln (L)=-\frac{n \pi}{2}-\frac{n}{2} \ln \left(\sigma_{\varepsilon}^{2}\right)-\frac{\left(\vec{y}^{(\lambda)}-X \vec{\kappa}\right)^{\prime} \cdot\left(\vec{y}^{(\lambda)}-X \vec{\kappa}\right)}{2 \sigma_{\varepsilon}^{2}}+(\lambda-1) \sum_{i=1}^{n} \ln y_{i} .
$$


No intuito de encontrar valores críticos de $\ell$, deriva-se a Eq. (3.17) em relação a $\kappa$ e $\sigma_{\varepsilon}^{2}$, o que resulta em:

$$
\begin{aligned}
\frac{\delta \ell}{\delta \kappa} & =\frac{1}{\sigma_{\varepsilon}^{2}} X^{\prime}\left(\vec{y}^{(\lambda)}-X \vec{\kappa}\right), \\
\frac{\delta \ell}{\delta \sigma_{\varepsilon}^{2}} & =-\frac{n}{2 \sigma_{\varepsilon}^{2}}+\frac{\left(\vec{y}^{(\lambda)}-X \vec{\kappa}\right)^{\prime} \cdot\left(\vec{y}^{(\lambda)}-X \vec{\kappa}\right)}{2\left(\sigma_{\varepsilon}^{2}\right)^{2}} .
\end{aligned}
$$

Ao se igualar estas equações a zero e substituir os resultados obtidos na Eq. (3.17) tem-se:

$$
\ell_{\max }(\lambda)=-\frac{n}{2} \ln \left(\hat{\sigma}^{2}\right)+(\lambda-1) \sum_{i=1}^{n} \ln y_{i}-\frac{n \pi}{2},
$$

em que

$$
\hat{\sigma}^{2}=\frac{\left(\vec{y}^{(\lambda)}-X \hat{\kappa}\right)^{\prime} \cdot\left(\vec{y}^{(\lambda)}-X \hat{\kappa}\right)}{n}=\frac{R(\lambda)}{n}, \text { e } \hat{\kappa}=\frac{X^{\prime} \vec{y}^{(\lambda)}}{X^{\prime} X} .
$$

onde $R(\lambda)$ é a soma de quadrados residuais de $y^{(\lambda)}$. A estimativa de máxima verossimilhança de $\hat{\lambda}$ será o valor de $\lambda$ que maximiza $\ell_{\max }(\lambda)$ ou, de forma equivalente, minimiza $R(\lambda)$.

Segundo Miranda [57], um caminho mais simples para obtenção deste parâmetro é utilizar a forma normalizada da transformação de Box-Cox da Eq. (3.12). Neste caso, o jacobiano da transformação é igual a 1 e, assim, o logaritmo da função de verossimilhança parcialmente maximizada pode ser escrito como:

$$
\ell_{\max }(\lambda)=-\frac{n}{2}\left(\ln \left(\hat{\sigma}^{2}\right)-\pi\right)
$$

equivalente à Eq. (3.20).

Apresentamos, assim, uma forma eficaz de utilização da função logaritmo generalizado nas transformações de Box-Cox. Diante da crescente utilização desta ferramenta, tanto na análise estatística tradicional, quanto em "Big Data" [58], notase que este procedimento facilita a notação e, muito mais do que isso, simplifica cálculos mediante utilização de propriedades inerentes à função $\ln _{\lambda}(t)$. Além disso, apresentamos como obter o valor ótimo do parâmetro da transformação para um dado conjunto de dados. 


\title{
4
}

\section{Uma Generalização para a Transformada de Fourier}

\author{
A Matemática parece ser uma faculdade da mente humana destinada \\ a ser suplementar a brevidade da vida e a imperfeição dos sentidos. \\ - Joseph Fourier
}

Uma importante ferramenta para transformação de dados e para o estudo de sinais é a transformada de Fourier (TF). A TF é uma transformada integral que expressa uma função em termos de senos e cossenos. Desde a apresentação da entropia generalizada de Tsallis [2], muitos pesquisadores se dedicaram à busca de uma generalização desta transformada. Contudo, eles esbarraram em alguns obstáculos para obter uma generalização mais consistente, como por exemplo, o pequeno intervalo no espaço do parâmetro $\lambda$. Neste capítulo, apresentamos uma nova versão generalizada da transformada de Fourier $(\lambda-\mathrm{TF})$ e a estendemos para incluir valores arbitrários do parâmetro $\lambda$. O procedimento envolve mapeamento conforme e fornece a conversão inversa de $\lambda$-TF, se essa transformação existir. Além disso, a extensão da $\lambda$-TF preserva a linearidade, generaliza a simetria de translação e outras importantes propriedades da TF usual, como, por exemplo, a transformada da derivada, a propriedade da modulação, a propriedade da conjugação, entre outras. Como aplicação, argumentamos que o parâmetro $\lambda$ pode ser extraído de sinais logperiódicos e que a distribuição delta de Dirac pode ser escrita em termos dessa $\lambda$-TF. Detalhamos aqui os resultados apresentados na Ref. [23].

Nos Caps. 2 e 3, foi apresentado que a função logaritmo generalizado desempenha um papel central no contexto da entropia não aditiva e é definida por 
$\ln _{\lambda}(t)=\left(t^{\lambda}-1\right) / \lambda$. Foi mostrado, ainda, que essa função descreve uma transformação de dados desenvolvida por Box e Cox [19, 20, 59]. A transformação Box-Cox implica que existe um valor adequado $\lambda$ a partir do qual os dados $\left\{x_{i}=x_{1}, x_{2}, \cdots, x_{n}\right\}$ são transformados por $y_{i}=\left(x_{i}^{\lambda}-1\right) / \lambda$ e passarão a ser distribuídos de acordo com uma função de densidade de probabilidade normal. Como resultado, métodos estatísticos paramétricos podem ser usados nos dados transformados. O mapeamento conforme aplicado na generalização da TF está intimamente ligado à concepção da transformação de Box-Cox. Neste caso, porém, o procedimento é transformado ao invés dos dados.

Outra estratégia comum na análise de dados emprega a transformada de Fourier trabalhando com a função característica da distribuição dos dados.

\subsection{A transformada de Fourier}

A transformada de Fourier faz parte de um grupo de transformadas conhecido como transformadas integrais. A transformada integral é um operador linear $\mathcal{T}$ escrito da seguinte forma:

$$
\mathcal{T}[f(t)]=g(u)=\int_{t_{1}}^{t_{2}} f(t) K(t, u) d t,
$$

em que $f(t)$ é a função a ser transformada e $K$ é o núcleo do operador, que varia de uma transformação para outra. Nesse grupo de transformações, incluem-se as transformadas de Laplace, Mellin, Hankel, Abel, Hilbert, wavelet entre outras.

O termo "transformada de Fourier" faz menção ao grande matemático e físico francês Jean Baptiste Joseph Fourier, que nasceu em Auxerre em 21 de março de 1768 e faleceu em Paris em 16 de maio de 1830. No reinado de Napoleão, exerceu vários cargos de cunho científico no Egito e na França, onde fundou a universidade que leva seu nome. Fourier teve muitos trabalhos reconhecidos, como, por exemplo, a teoria analítica do calor, teorema de Fourier sobre raízes reais de polinômios (póstumo) e a transformada de Fourier. Essa transformação decompõe uma função do tempo em suas frequências constituintes, geralmente em termos de senos e cossenos [60]. De modo geral, dados $f$ e $g$, funções reais (ou complexas), a transformada de Fourier, designada aqui como $\mathcal{F}$, pode ser definida da seguinte maneira:

$$
\mathcal{F}[f(t)]=g(\omega) \equiv \frac{1}{\sqrt{2 \pi}} \int_{-\infty}^{\infty} f(t) \mathrm{e}^{-i \omega t} d t .
$$


Nesta transformada, e $e^{i \omega t}$ é o núcleo. A inversa desta transformação, quando existir, é escrita como

$$
\mathcal{F}^{-1}[g(\omega)]=f(t) \equiv \frac{1}{\sqrt{2 \pi}} \int_{-\infty}^{\infty} g(\omega) \mathrm{e}^{i \omega t} d t .
$$

A transformada de Fourier possui as seguintes propriedades:

1. Linearidade: Para quaisquer $\alpha_{1}, \alpha_{2} \in \mathbb{C}$, se $f(t)=\alpha_{1} h_{1}(t)+\alpha_{2} h_{2}(t)$ então:

$$
\mathcal{F}[f(t)]=\mathcal{F}\left[\alpha_{1} h_{1}(t)+\alpha_{2} h_{2}(t)\right]=\alpha_{1} \mathcal{F}\left[h_{1}(t)\right]+\alpha_{2} \mathcal{F}\left[h_{2}(t)\right] .
$$

Essa propriedade é fundamental para que a Transformada de Fourier pertença à família das transformadas integrais.

2. Translação: para $\forall a \in \mathbb{C}$ tem-se que:

$$
\mathcal{F}[f(t \pm a)]=\mathrm{e}^{ \pm i a \omega} \mathcal{F}[f(t)]
$$

3. Conjugação: dada $f(t)$, função real, e $\mathcal{F}[f(t)]$ sua respectiva transformada de Fourier, então:

$$
\mathcal{F}[-f(t)]=\overline{\mathcal{F}[f(t)]},
$$

em que $\overline{\mathcal{F}[f(t)]}$ é o complexo conjugado de $\mathcal{F}[f(t)]$.

4. Transformada da Derivada: dada uma função diferenciável $f(t)$ tal que $\lim _{t \rightarrow \pm \infty} f(t)=0$, e sua TF $\mathcal{F}[f(t)]$, então

$$
\mathcal{F}\left[f^{\prime}(t)\right]=i \omega \mathcal{F}[f(t)]
$$

5. Transformada da Integral: dada uma função integrável $f(t)$ tal que sua transformada de Fourier $\mathcal{F}[f(t)]$ satisfaça $\mathcal{F}[0]=0$, então:

$$
\mathcal{F}\left[\int_{-\infty}^{t} f(\tau) d \tau\right]=\frac{1}{i \omega} \mathcal{F}[f(t)]
$$

6. Modulação: dada uma função $f(t)$, com $f\left(t_{0}\right)=\omega_{0}$, e $\mathcal{F}[f(t)]=F(\omega)$ sua respectiva $\mathrm{TF}$, então, vale a seguinte propriedade

$$
\mathcal{F}\left[f(t) \cos \left(\omega_{0} t\right)\right]=\frac{1}{2} F\left(\omega+\omega_{0}\right)+\frac{1}{2} F\left(\omega-\omega_{0}\right) .
$$

7. Convolução: dadas as funções $f_{1}(t)$ e $f_{2}(t)$ com suas respectivas transformadas de Fourier $\mathcal{F}_{1}\left[f_{1}(t)\right]$ e $\mathcal{F}_{2}\left[f_{2}(t)\right]$, então, a convolução no tempo é dada por

$$
\mathcal{F}\left[\left(f_{1} * f_{2}\right)(t)\right]=\mathcal{F}_{1}\left[f_{1}(t)\right] \mathcal{F}_{2}\left[f_{2}(t)\right]
$$


Por outro lado, no campo da frequência,

$$
\left(\mathcal{F}_{1}\left[f_{1}(t)\right] * \mathcal{F}_{2}\left[f_{2}(t)\right]\right)=2 \pi \mathcal{F}\left[f_{1}(t) f_{2}(t)\right],
$$

em que $*$ representa a convolução entre duas funções, de tal forma que

$$
\left(f_{1} * f_{2}\right)(t)=\int_{-\infty}^{\infty} f_{1}(\tau) f_{2}(t-\tau) d \tau
$$

8. Teorema de Parseval: seja $f(t)$ uma função e $\mathcal{F}$, sua transformada de Fourier, então vale a propriedade:

$$
\int_{-\infty}^{\infty}|f(t)|^{2} d t=\frac{1}{2 \pi} \int_{-\infty}^{\infty}|\mathcal{F}[f(t)]|^{2} d \omega
$$

9. Princípio da Incerteza: seja $f(t)$ uma função real de tal forma que $\lim _{t \pm \infty} f(t)=$ 0 e $\mathcal{F}[f(t)]$ sua TF. Então vale a estimativa:

$$
\int_{-\infty}^{\infty}|t f(t)|^{2} d t \int_{-\infty}^{\infty}|f(t) \mathcal{F}[f(t)]|^{2} d \omega \geq\left.\left.\frac{\pi}{2}\left|\int_{-\infty}^{\infty}\right| f(t)\right|^{2} d t\right|^{2} .
$$

Dada a importância da Transformada de Fourier e suas aplicações nas mais diversas áreas, muitos autores têm dedicado tempo e estudo na busca por uma generalização consistente. Na próxima seção, apresentamos algumas dessas tentativas e propomos uma nova generalização.

\subsection{Generalizando a transformada de Fourier}

A generalização da transformada de Fourier é um tema recorrente entre estudiosos de diversas áreas. Em 1933, por exemplo, Wiener [61] apresentou uma generalização baseada em conceitos ligados a polinômios Hermitianos. Mais recentemente, em 1996, Askey [62] apresenta uma proposta que também se utiliza de uma decomposição polinomial. A partir do surgimento da entropia generalizada e, com ela, a generalização da função exponencial $\left(\mathrm{e}_{\lambda}(t)=\lim _{\lambda^{\prime} \rightarrow \lambda}\left(1+\lambda^{\prime} t\right)^{1 / \lambda^{\prime}}\right)$, apresentada no Cap. 2, surgiu um outro tipo de generalização desta transformada, a que utiliza a função $e_{\lambda}(t)$ como núcleo da transformação. Normalmente, tais generalizações são referenciadas como $q$-Transformadas de Fourier, em virtude do parâmetro $q$. Aqui utiliza-se a nomenclatura $\lambda$-Transformada de Fourier $(\lambda$-TF) acompanhando a notação adotada $(\lambda=1-q)$.

No contexto das $\lambda$-TFs, existem vários modelos apresentados. Contudo, muitas generalizações apresentam restrições referentes ao domínio, ao parâmetro $\lambda$ ou 
ainda, em relação à inversibilidade da transformada. Além disso, muitas dessas transformações acabam não preservando propriedades e características importantes da TF original.

Umarov e Tsallis [63] apresentam uma $\lambda$-TF que lida muito bem com funções generalizadas. Contudo, tal transformação só é valida para $\lambda$ em um restrito intervalo: $-2<\lambda \leqslant 0$. Nesta definição, tem-se que, se $f(t)$ é uma função não negativa, a sua $\lambda$-TF é dada por

$$
F_{\lambda}[f(t)]=\int_{-\infty}^{\infty} \mathrm{e}_{\lambda}(i \omega t) \otimes_{\lambda} f(t) d t
$$

em que $\otimes_{\lambda}$ é o produto generalizado definido como $a \otimes b=\left(a^{\lambda}+b^{\lambda}-1\right)^{1 / \lambda}$. Maiores detalhes desse operador podem ser observados na Sec. 2.4 do Cap. 2.

Na Eq. (4.15), é possível observar que $e_{\lambda}(t)$ é estendida para o plano complexo de forma direta, ou seja, a unidade imaginária é acoplada diretamente no argumento principal da generalização. Mesmo assim, o caso particular com $\lambda=0$ recupera a transformada de Fourier usual. Para outros valores de $\lambda$ no intervalo ]-2,0[ tem-se uma transformação não linear, já que $[f(t)+g(t)] \otimes_{\lambda} \mathrm{e}_{\lambda}(i \omega t)=[f(t)+$ $g(t)] \mathrm{e}_{\lambda}\left(i \omega t[f(t)+g(t)]^{-\lambda}\right)$, de modo que $F[f+g] \neq F[f]+F[g]$. Esse detalhe tira a possibilidade de classificar esta transformação como uma transformada integral, para $\lambda \neq 0$.

Outro problema enfrentado pela Eq. (4.15) envolve as restrições em vigor para $\lambda$. Tendo em mente a transformação de dados Box-Cox (apresentada no Cap. 3), $\lambda$ transforma um conjunto de dados observado $x_{i}$ em um normalmente distribuído, $y_{i}^{(\lambda)}$. Portanto, $\lambda$ depende dos próprios dados observados e pode eventualmente adquirir valores fora do intervalo de validade. Além disso, para $\lambda>0$, o produto generalizado está mal definido, já que o "zero" não se comporta como o elemento nulo, conforme mostrado na Sec. 2.4 do Cap. 2.

Outra questão a ser discutida tem foco no inverso desta $\lambda$-TF. Vários estudos abordaram tal relação (inversa) para funções específicas $f(t)$ e intervalos específicos para $\lambda[41,42]$. No entanto, Hilhorst [64] argumenta que a Eq. (4.15) não possui um inverso adequado, em geral, porque pode produzir o mesmo resultado para funções distintas $f(t)$. Esse cenário cria uma degeneração não intencional na $\lambda$-TF, que por sua vez proíbe o cálculo da transformada inversa. Esse problema é causado pelo aspecto não linear da Eq. (4.15), o que dificulta a aplicação de $\lambda$-TF para dados 
gerais. Definições alternativas para a $\lambda$-TF e seu inverso foram apresentadas por outros autores $[65,66]$. Contudo, nestas referências existe a restrição de $\lambda$ em um intervalo ainda menor: $-1<\lambda \leq 0$.

Uma outra maneira de generalizar a TF envolve transformações lineares homomorficamente equivalentes à transformada usual. Em um trabalho recente, Kalogeropoulos [18] explora essa opção definindo a $\tau_{q}$-Transformada de Fourier invertível. Essa transformada utiliza o $\lambda$-produto alternativo $[15,17]$ apresentado na Sec. 2.4 do Cap. 2 como $\odot_{\lambda}$ em detrimento a $\otimes_{\lambda}$ utilizado na Eq. (4.15). A vantagem obtida nesta "troca" de operadores é que $\odot_{\lambda}$ é distributivo em relação à $\lambda$-soma, criando assim uma estrutura de anel generalizado $\left(\mathbb{R}_{\lambda}, \oplus_{\lambda}, \odot_{\lambda}\right)$, já apontado na Sec. 2.4.

Na mesma linha, Scarfone [67] obtém uma transformada de Fourier generalizada invertível. A grande diferença é que, neste caso, dentre outras conclusões importantes, a generalização da Transformada de Fourier tem no núcleo uma expressão na qual a unidade imaginária aparece como potência da exponencial generalizada, diferentemente da definição apresentada na Eq. (4.15), onde o número imaginário faz parte do argumento.

$$
\mathbb{F}_{\lambda}[f(t)]=\frac{1}{\sqrt{2 \pi}} \int_{-\infty}^{\infty} f(t)\left[\mathrm{e}_{\lambda}\left(-\omega \odot_{\lambda} t\right)\right]^{i} d t
$$

Diante desses avanços apresentados em publicações recentes, este estudo também se concentra nas transformações homomórficas, pois elas preservam a linearidade e são invertíveis. Desta forma, utilizamos os mapeamentos conformes como uma maneira de alcançar transformações homomórficas.

\subsection{Mapeamento e mapeamento conforme}

Na Matemática, o termo mapa ou "mapeamento" faz menção a uma função (ou transformação) que "transfere" uma estrutura de um "espaço" (ou sistema de coordenadas) para outro. É uma ferramenta muito utilizada, já que, em 1851, Riemann demonstrava um teorema que afirma que uma região delimitada do plano pode ser mapeada por um círculo [68]. Depois dele, outros nomes bem conhecidos trabalharam neste tema, como por exemplo, Schwarz (1843-1921), A. Harnack (1851-1888) e H. Poincaré(1854-1912). Em geral, utiliza-se esse recurso para simplificar cálculos e notações. A mudança de coordenadas, de uma curva ou função, de cartesianas para cilíndricas ou esféricas é um exemplo de mapeamento. 
Mapeamento é um recurso muito utilizado na análise complexa, em que, dada uma função $w$ e um número $z \in \mathbb{C}$, de tal forma que

$$
w=f(z)=u(x, y)+i v(x, y)
$$

é uma função que leva pontos do plano complexo $z$ para pontos no plano complexo $w$ e, por extensão, curvas no plano $z$ são "mapeadas" para curvas no plano $w$ [69]. A Fig. (4.1) apresenta como exemplo de mapeamento $w=f(z)=z^{2}$. Se $z=x+i y$, então $f(z)$ pode ser reescrita na forma $f(z)=u(x, y)+i v(x, y) \operatorname{com} u(x, y)=x^{2}-y^{2}$ e $v(x, y)=2 x y$.

$$
f(z)=z^{2}
$$

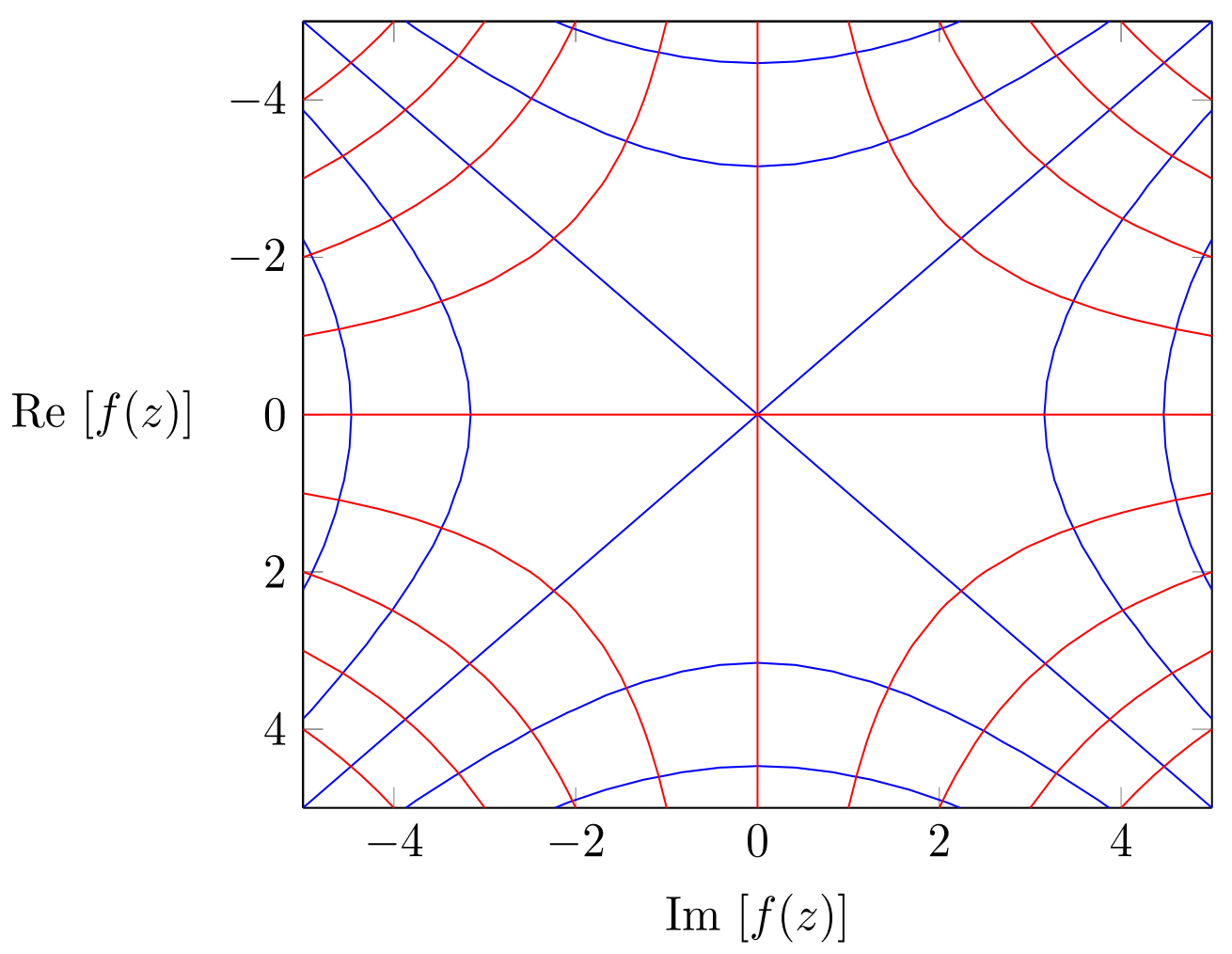

Figura 4.1: Exemplo de mapeamento com $w=f(z)=z^{2}$. Se $z$ é tal que $z=x+i y$, então $f(z)=u(x, y)+i v(x, y) \operatorname{com} u(x, y)=x^{2}-y^{2}$ e $v(x, y)=2 x y$.

Um mapeamento conforme, também conhecido como mapa ou transformada conforme é uma transformação $w=f(z)$ que preserva ângulos (ou áreas) locais. Uma propriedade destes mapas é que uma função analítica é conforme em qualquer ponto cuja derivada não é nula. Por outro lado, qualquer mapeamento conforme de uma variável complexa que tenha derivadas parciais contínuas é analítico. Isso 
traz grandes vantagens na análise complexa, já que funções analíticas garantem um conjunto de propriedades, fugindo do estudo de ramos e singularidades. Outra propriedade interessante de transformações conformes é que dado $w=f(z)$, conforme, então

$$
\left|f^{\prime}\left(z_{0}\right)\right|=\left|\lim _{z \rightarrow z_{0}} \frac{f(z)-f\left(z_{0}\right)}{z-z_{0}}\right|=\lim _{z \rightarrow z_{0}} \frac{\left|f(z)-f\left(z_{0}\right)\right|}{\left|z-z_{0}\right|},
$$

em que $z_{0}$ é um ponto qualquer e $f^{\prime}\left(z_{0}\right)$ é a derivada de $w$ neste ponto. Desta equação, pode-se indicar que $\left|f^{\prime}\left(z_{0}\right)\right|$ representa uma expansão se for maior que 1 e uma retração se menor que 1 . Assim, a imagem de uma pequena região na vizinhança de $z_{0}$ é conforme à região original no sentido de ter, aproximadamente, o mesmo formato. O mapa apresentado na Fig. (4.1) é um mapeamento conforme.

Uma outra propriedade é que se $w=f(z)$ for conforme em um ponto $z=z_{0}$, então $w$ tem uma inversa local neste ponto. Em outras palavras, se $w_{0}=f\left(z_{0}\right)$, então existe uma única transformação $z=g(w)$, definida e analítica em uma vizinhança $N$ de $w_{0}$, tal que $g\left(w_{0}\right)=z_{0}$ e $f[g(w)]=1$ em cada ponto $w$ de $N$. Além disso, a derivada de $g(w)$ é

$$
g^{\prime}(w)=\frac{1}{f^{\prime}(z)} .
$$

Um bom exemplo de mapeamento conforme é a transformação $w=f(z)=\mathrm{e}^{z}$, para todo $z \in \mathbb{C}$, já que $w$ é analítica, sua derivada é não nula em todo plano complexo. A inversa de $w=f(z)=\mathrm{e}^{z}$ é $z=g(w)=\log (w)=\ln \rho+i \phi(\rho>0, \pi<$ $\theta<3 \pi)$, restrito a qualquer vizinhança de $w_{0}$ que não contenha a origem. De fato, tomando por exemplo, $z_{0}=2 \pi i$, tem-se que a imagem de $z_{0}$ é $w_{0}=1$, por outro lado, tomando $w_{0}=1$, em $z=g(w)$ recuperamos $2 \pi i$.

O mapeamento conforme é extremamente importante em análise complexa, física, engenharias e em outras áreas do conhecimento, já que é uma ferramenta eficaz na resolução da equação de Laplace para problemas de eletrostática, hidrodinâmica, fluxo do calor e assim por diante [43]. Outros detalhes sobre este tema podem ser obtidos na Ref. [70].

\subsection{Uma nova extensão da $\lambda$-Transformada de Fourier}

Transformações integrais são geralmente definidas pelos seus núcleos. No caso da transformada de Fourier, o núcleo é uma função exponencial simples e ${ }^{i \omega t}$ na 
qual $\omega$ e $t$ têm dimensões recíprocas. De acordo com a discussão apresentada no final da Sec. 2.5 do Cap. 2, a função exponencial convencional e $(t)=\mathrm{e}_{0}(t)$, satisfaz as igualdades $\mathrm{e}^{i \omega t}=\left(\mathrm{e}^{\omega t}\right)^{i}=\left(\mathrm{e}^{t}\right)^{i \omega}$. A partir dessa inofensiva degenerescência, surge uma questão para definir uma generalização da $\mathrm{TF}$, já que $\mathrm{e}_{\lambda}(i \omega t) \neq\left[\mathrm{e}_{\lambda}(\omega t)\right]^{i} \neq$ $\left[\mathrm{e}_{\lambda}(t)\right]^{i \omega}$ e seu comportamento oscilatório muda drasticamente de um para outro. Por exemplo, $\operatorname{Re}\left[\mathrm{e}_{1}(i \omega t)\right]=1$, enquanto $\operatorname{Re}\left[\mathrm{e}_{1}(\omega t)^{i}\right]$ e $\operatorname{Re}\left[\mathrm{e}_{1}(t)^{i \omega}\right]$ oscilam para $t, \omega \geq 0$, embora com frequências muito distintas. Essas situações são ilustradas na Fig. (4.2).

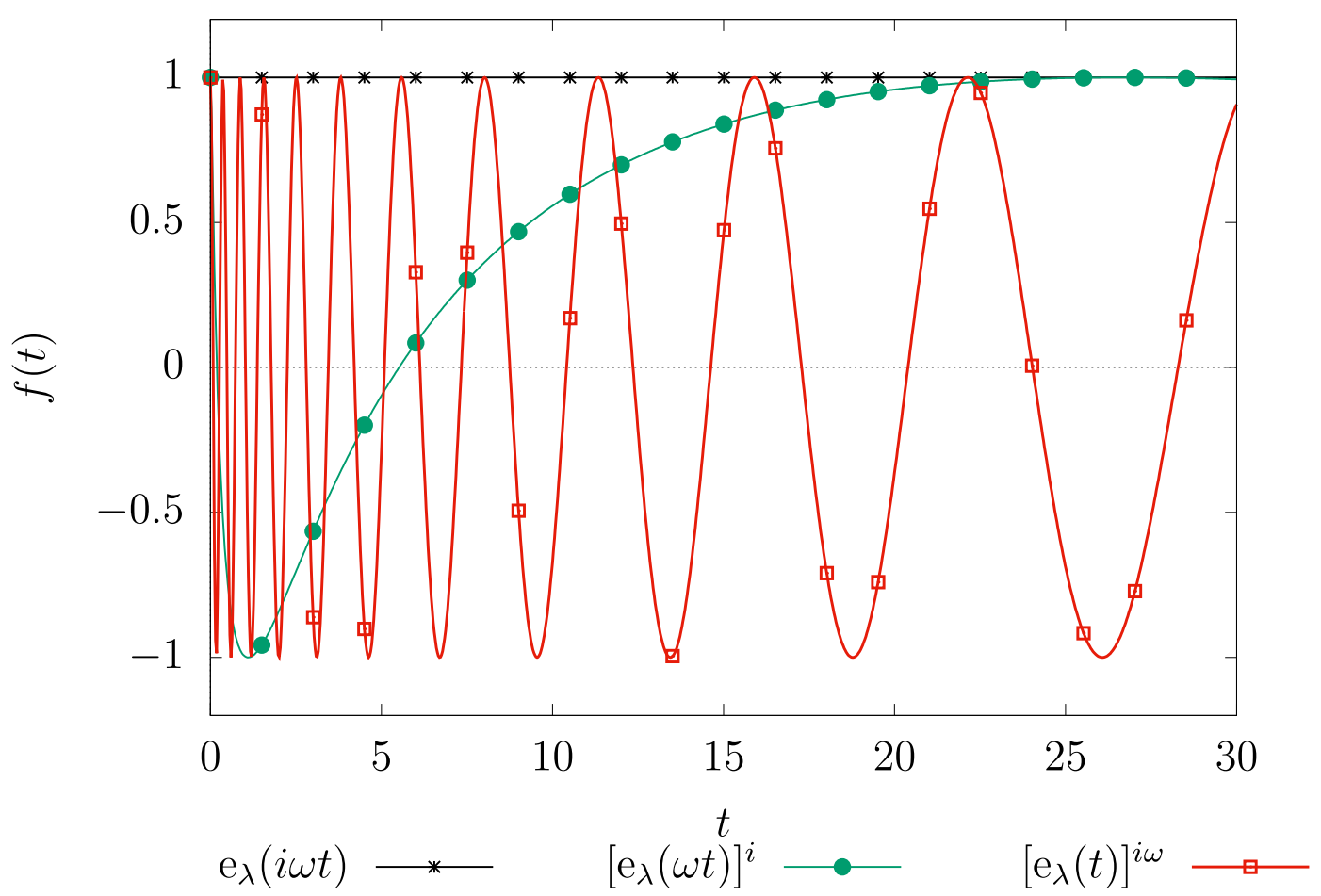

Figura 4.2: Diferentes possibilidades para a generalização de $f(t)=e^{i \omega t}: \mathrm{e}_{\lambda}(i \omega t),\left[\mathrm{e}_{\lambda}(\omega t)\right]^{i} \mathrm{e}$ finalmente $\left[\mathrm{e}_{\lambda}(t)\right]^{i \omega}$. Exemplo para cada uma das curvas com $t \geq 0, \lambda=1 \mathrm{e} \omega=20$. Curvas oscilam, com frequências bem diferentes, para $\left[\mathrm{e}_{\lambda}(\omega t)\right]^{i} \mathrm{e}\left[\mathrm{e}_{\lambda}(t)\right]^{i \omega}$. Para $\mathrm{e}_{\lambda}(i \omega t)$ tem-se uma função constante.

Diante destes questionamentos, é preciso selecionar um núcleo consistente com a descrição dos dados desejados. Em definições anteriores, observa-se que o núcleo utilizado foi, na maioria das citações, $\mathrm{e}_{\lambda}(i \omega t)[41,42,65,66,71]$. Contudo, nota-se que $\left[\mathrm{e}_{\lambda}(t)\right]^{i \omega}$ é mais natural e pode reproduzir oscilações de variação rápida e lenta, incluindo oscilações log-periódicas, muito mais adequadas para aplicações relacionadas à invariância em escala discreta, variando de análise financeira a pro- 
cessos de fratura $[24,72]$. Por esse motivo, mantém-se atenção apenas em $\left[\mathrm{e}_{\lambda}(t)\right]^{i \omega}$.

Um problema detectado nessa escolha diz respeito à análise de dimensões, já que não tem-se mais um produto entre variáveis com dimensões recíprocas. Para superar esse entrave, permite-se que tanto o parâmetro $\lambda$, quanto as demais variáveis $t$ e $\omega$ sejam adimensionais.

Com todas as disposições preliminares já instauradas, propõe-se uma nova generalização para a transformada de Fourier - a $\lambda$-TF:

$$
\mathcal{F}_{\lambda}[f]=\frac{1}{\sqrt{4 \pi}} \int_{-\infty}^{\infty} \frac{\left[\mathrm{e}_{\lambda}(t)\right]^{i \omega}}{|1+\lambda t|} f(t) d t .
$$

A Eq.(4.20) estende o conceito da $\lambda$-TF, $\forall \lambda \in \mathbb{R}$ de forma muito satisfatória, já que, tendo em mente as discussões acerca de generalizações consistentes do Cap. 2 e também presente no decorrer do trabalho, ela preserva características e propriedades importantes da TF original. Em primeiro lugar, quando $\lambda \rightarrow 0$ recupera-se a TF usual. Além disso, a $\lambda$-TF apresentada na Eq. (4.20) preserva a linearidade, já que

$$
\begin{aligned}
\mathcal{F}_{\lambda}\left[a_{1} f_{1}+a_{2} f_{2}\right] & =\frac{1}{\sqrt{4 \pi}} \int_{-\infty}^{\infty} \frac{\left[\mathrm{e}_{\lambda}(t)\right]^{i \omega}}{|1+\lambda t|}\left[a_{1} f_{1}+a_{2} f_{2}\right](t) d t= \\
& =\frac{1}{\sqrt{4 \pi}}\left[a_{1} \int_{-\infty}^{\infty} \frac{\left[\mathrm{e}_{\lambda}(t)\right]^{i \omega}}{|1+\lambda t|} f_{1}(t) d t+a_{2} \int_{-\infty}^{\infty} \frac{\left[\mathrm{e}_{\lambda}(t)\right]^{i \omega}}{|1+\lambda t|} f_{2}(t)\right]= \\
& =a_{1} \mathcal{F}_{\lambda}\left[f_{1}\right]+a_{2} \mathcal{F}_{\lambda}\left[f_{2}\right] .
\end{aligned}
$$

Este fato credencia a Eq. (4.20) como uma transformada integral generalizada sob o parâmetro $\lambda$, conforme indicado na Sec. 4.1.

Em geral, a avaliação explícita da Eq. (4.20) atende à maioria das aplicações e exigências para uma TF, porém fornece pouco conhecimento sobre a lógica por trás de si mesma. A introdução de um mapa conforme pode ajudar a esclarecer este fato, já que com ele é possível explicitar um relacionamento entre a Eq.(4.20) com $0-\mathrm{TF}$.

Sem perder a generalidade, pode-se assumir $\lambda, \omega \geq 0$. Seja ainda, $z$ uma variável complexa. Um mapeamento conforme entre $z$ e $t$ é definido por

$$
z=\frac{1}{\lambda} \ln (1+\lambda t)=\ln \left[\mathrm{e}_{\lambda}(t)\right]
$$

A Fig. 4.3 descreve este mapeamento e destaca dois recursos importantes. O primeiro recurso é que a Eq.(4.22) mapeia todo o $\mathbb{R}$ em duas linhas paralelas, 
separadas pela distância $d=\pi / \lambda$. Mais especificamente, a parte imaginária de $z$ desaparece para $t \geq t_{c} \equiv-1 / \lambda$; enquanto que para $t<t_{c}$ sempre se pode reescrever $\mathrm{e}^{ \pm i \pi}|1+\lambda t|$ a fim de que $\lambda z= \pm i \pi+\ln |1+\lambda t|$. O segundo recurso é que este mapeamento reforça a relação $\mathrm{e}^{z}=\mathrm{e}_{\lambda}(t)$, já apontada em outra discussão na Sec. 2.6 do Cap. 2. Em outras palavras, as funções exponenciais muito mais familiares em $z$ correspondem às funções $\lambda$-exponenciais em $t$. Como as funções exponenciais são componentes importantes das transformações integrais (transformada de Fourier e Laplace, etc.), o mapeamento também pode ser explorado para criar suas representações no contexto de funções generalizadas.

a)

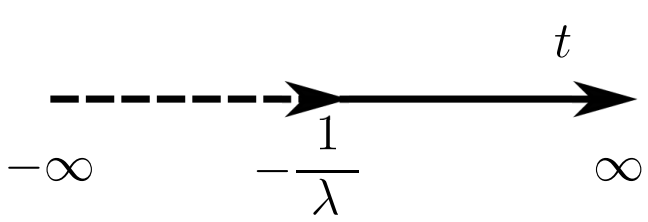

b)

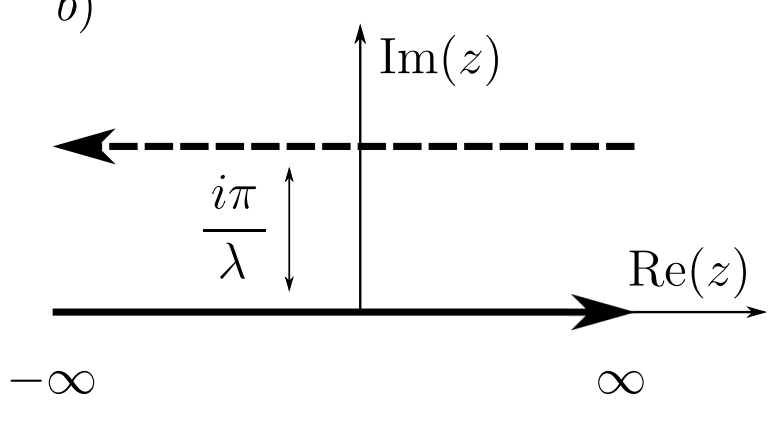

Figura 4.3: O logaritmo natural mapeia um segmento de linha reta em dois segmentos de linha paralelos com direções opostas no plano complexo. (a) O primeiro segmento de linha (tracejado) varia de $-\infty$ a $t_{c}$, enquanto o segundo segmento de linha começa em $t_{c}$ e continua até $\infty$. (b) $\mathrm{O}$ mapa conforme estende o primeiro segmento de linha em a) e o desloca por $i \pi / \lambda$ (tracejado).

O próximo passo trata da integral na Eq. (4.20) e sua representação usando a variável z. Por uma questão de clareza e em concordância com a Fig. (4.3 a) pode-se separá-la em duas contribuições,

$$
\mathcal{F}_{\lambda}[f]=\frac{1}{\sqrt{4 \pi}} \int_{-\infty}^{-\frac{1}{\lambda}} \frac{\left[\mathrm{e}_{\lambda}(t)\right]^{i \omega}}{|1+\lambda t|} f(t) d t+\frac{1}{\sqrt{4 \pi}} \int_{-\frac{1}{\lambda}}^{\infty} \frac{\left[\mathrm{e}_{\lambda}(t)\right]^{i \omega}}{|1+\lambda t|} f(t) d t .
$$

A primeira integral no lado direito da equação corresponde à linha em que $\operatorname{Im}(z)=$ $\pi / \lambda$ (linha pontilhada na Fig. (4.3 b)). Já a segunda produz contribuições relacionadas ao caminho ao longo do qual a parte imaginária de $z$ se anula, ou seja, $\operatorname{Im}(z)=0$ (linha contínua na Fig(4.3 b)).

De modo rápido, apenas para se ter uma ideia da simplificação fornecida pelo mapeamento proposto na Eq. (4.22), tem-se que,

$$
\int_{-\frac{1}{\lambda}}^{\infty} \frac{\left[\mathrm{e}_{\lambda}(t)\right]^{i \omega}}{|1+\lambda t|} f(t) d t \stackrel{z=\frac{1}{\lambda} \ln (1+\lambda t)}{\longrightarrow} \int_{-\infty}^{\infty} \frac{\mathrm{e}^{i \omega z}}{|1+\lambda t|} f(\tilde{z}) J(t) d z
$$


em que $J(t)=(1+\lambda t)$ é o jacobiano da transformação e $\tilde{z}=\ln _{\lambda}\left(\mathrm{e}^{z}\right)$. Como no intervalo $-1 / \lambda \leq t<\infty$, a expressão $1+\lambda t$ produz apenas valores positivos, a Eq. (4.24) pode ser escrita, de forma simplificada como

$$
\int_{-\frac{1}{\lambda}}^{\infty} \frac{\left[\mathrm{e}_{\lambda}(t)\right]^{i \omega}}{|1+\lambda t|} f(t) d t \equiv \int_{-\infty}^{\infty} \mathrm{e}^{i \omega z} f(\tilde{z}) d z .
$$

De volta a Eq. (4.23), pode-se trabalhar com uma expressão um pouco mais limpa e, além disso, reforçar que as diferentes contribuições das integrais surgem de diferentes caminhos. Para tanto, é conveniente definir $g_{1}(z) \equiv f(t) \theta\left(t-t_{c}\right)$ e $g_{2}(z) \equiv f(t) \theta\left(t_{c}-t\right)$, em que $\theta(x)$ é a função teta de Heaviside (função degrau, $\theta(x \geq 0)=1$ e $\theta(x<0)=0)$. Desse modo, uma equação muito mais clara pode ser expressa em termos da transformada de Fourier usual:

$$
\begin{aligned}
\mathcal{F}_{\lambda}[f] & =\frac{1}{\sqrt{4 \pi}} \int_{-\infty}^{\infty} \mathrm{e}^{i \omega z} g_{1}(z) d z+\frac{\mathrm{e}^{-\pi \omega / \lambda}}{\sqrt{4 \pi}} \int_{-\infty}^{\infty} \mathrm{e}^{i \omega z} g_{2}(z) d z \\
& =\frac{1}{\sqrt{2}}\left[\tilde{g}_{1}(\omega)+\mathrm{e}^{-\pi \omega / \lambda} \tilde{g}_{2}(\omega)\right],
\end{aligned}
$$

com $\tilde{g}_{1}(\omega)$ e $\tilde{g}_{2}(\omega)$ sendo a 0 -TF de $g_{1}(z)$ e $g_{2}(z)$, respectivamente. Portanto, se $\tilde{g}_{1}(\omega)$ e $\tilde{g}_{2}(\omega)$ existem, então $\mathcal{F}_{\lambda}[f]$ também existe.

O mapeamento conforme na Eq. (4.22) fornece uma ponte entre a $\lambda$-TF apresentada na Eq. (4.20) com a TF usual da Eq. (4.26). Este mapeamento compartilha diretrizes semelhantes às do método Box-Cox: procura uma transformação variável que simplifique ou permita a análise tradicional nos dados transformados. No entanto, aqui, o próprio procedimento (Fourier) é transformado em vez dos dados.

\subsection{Distribuição $\lambda$-Delta}

A "função" delta de Dirac é uma distribuição introduzida pelo físico Paul Dirac. É utilizada para modelar a densidade de uma massa (ou carga) pontual ou ideal sendo nula em qualquer ponto exceto na origem. Como uma distribuição, a delta de Dirac é um funcional linear que mapeia todas as funções para seu valor em zero. Em engenharia e processamento de sinais, a delta, também conhecida como símbolo de impulso unitário, pode ser considerada por meio de sua transformada de Laplace, como proveniente dos valores-limite de uma função analítica complexa. Essa função possui transformada de Fourier bem definida, à saber

$$
\mathcal{F}[\delta(t)]=\int_{-\infty}^{\infty} \mathrm{e}^{-i \omega t} \delta(t) d t=1,
$$


como também a transformada inversa,

$$
\mathcal{F}^{-1}[\delta(t)]=\mathcal{F}[1]=\int_{-\infty}^{\infty} \mathrm{e}^{i \omega t}=\delta(t)
$$

Por esse "bom comportamento" da distribuição delta e principalmente por sua aplicabilidade em várias áreas da ciência, vamos apresentar como primeiro exemplo, a $\lambda$-TF da Eq. (4.20). Para isso, considere $f(t)=1$ :

$$
\begin{aligned}
\mathcal{F}_{\lambda}[1] & =\frac{1}{\sqrt{4 \pi}} \int_{-\infty}^{\infty} \frac{\left[\mathrm{e}_{\lambda}(t)\right]^{i \omega}}{|1+\lambda t|} 1 d t \equiv \\
& \equiv \frac{1}{\sqrt{4 \pi}} \int_{-\infty}^{\infty} \mathrm{e}^{i \omega z} 1 d z+\frac{\mathrm{e}^{-\pi \omega / \lambda}}{\sqrt{4 \pi}} \int_{-\infty}^{\infty} \mathrm{e}^{i \omega z} 1 d z= \\
& =\left(\frac{1+\mathrm{e}^{-\pi \omega / \lambda}}{\sqrt{4 \pi}}\right) \lim _{R \rightarrow \infty} \int_{-R}^{R} \mathrm{e}^{i \omega z} d z= \\
& =\left(\frac{1+\mathrm{e}^{-\pi \omega / \lambda}}{\sqrt{\pi}}\right) \lim _{R \rightarrow \infty} \frac{\operatorname{sen}(\omega R)}{\omega}=\left(\frac{1+\mathrm{e}^{-\pi \omega / \lambda}}{\sqrt{\pi}}\right) \pi \delta(\omega)= \\
& =\left(1+\mathrm{e}^{-\pi \omega / \lambda}\right) \sqrt{\pi} \delta(\omega)=\sqrt{4 \pi} \delta_{\lambda}(\omega) .
\end{aligned}
$$

Este resultado mostra que a generalização proposta segue o comportamento da TF usual para qualquer valor do parâmetro $\lambda$. Além disso, a Eq. (4.29), apresenta uma possibilidade de representação da distribuição delta de Dirac $\delta(\omega)$ em termos da $\lambda$-TF proposta:

$$
\delta_{\lambda}(\omega)=\frac{1}{4 \pi} \int_{-\infty}^{\infty} \frac{\left[\mathrm{e}_{\lambda}(t)\right]^{i \omega}}{|1+\lambda t|} d t
$$

Outro exemplo é a transformação de $f(t)=\left[\mathrm{e}_{\lambda}(t)\right]^{i \omega_{0}}$. Nesse caso, uma analogia com a transformada de Fourier usual sugere que o resultado deve ser proporcional a $\delta_{\lambda}\left(\omega+\omega_{0}\right)$. De fato, temos a seguinte relação:

$$
\begin{aligned}
\mathcal{F}_{\lambda}\left[\mathrm{e}_{\lambda}(t)^{i \omega_{0}}\right] & =\frac{1}{\sqrt{4 \pi}} \int_{-\infty}^{\infty} \frac{\left[\mathrm{e}_{\lambda}(t)\right]^{i \omega}}{|1+\lambda t|}\left[\mathrm{e}_{\lambda}(t)\right]^{i \omega_{0}} d t= \\
& =\frac{1}{\sqrt{4 \pi}} \int_{-\infty}^{\infty} \frac{\left[\mathrm{e}_{\lambda}(t)\right]^{i\left(\omega+\omega_{0}\right)}}{|1+\lambda t|} d t \equiv \\
& \equiv \frac{1}{\sqrt{4 \pi}} \int_{-\infty}^{\infty} \mathrm{e}^{i\left(\omega+\omega_{0}\right) z} d z+\frac{\mathrm{e}^{-\pi\left(\omega+\omega_{0}\right) / \lambda}}{\sqrt{4 \pi}} \int_{-\infty}^{\infty} \mathrm{e}^{i\left(\omega+\omega_{0}\right) z} d z= \\
& =\left(\frac{1+\mathrm{e}^{-\pi\left(\omega+\omega_{0}\right) / \lambda}}{\sqrt{4 \pi}}\right) \lim _{R \rightarrow \infty} \int_{-R}^{R} \mathrm{e}^{i\left(\omega+\omega_{0}\right) z} d z= \\
& =\left(\frac{1+\mathrm{e}^{-\pi\left(\omega+\omega_{0}\right) / \lambda}}{\sqrt{\pi}}\right) \lim _{R \rightarrow \infty} \frac{\operatorname{sen}\left[\left(\omega+\omega_{0}\right) R\right]}{\omega+\omega_{0}}= \\
& =\left(1+\mathrm{e}^{-\pi\left(\omega+\omega_{0}\right) / \lambda}\right) \sqrt{\pi} \delta_{\lambda}\left(\omega+\omega_{0}\right)=\sqrt{4 \pi} \delta_{\lambda}\left(\omega+\omega_{0}\right) .
\end{aligned}
$$


Desta forma, verifica-se em mais um exemplo que a $\lambda$-TF proposta acompanha os resultados da TF convencional. Isso indica que o caminho seguido para chegar a esta generalização foi muito bem pensado e estruturado, já que até aqui essa proposta tem obedecido às principais propriedades e características da TF original. À seguir, são apresentadas mais algumas propriedades válidas para esta $\lambda$-TF.

\subsection{Translações generalizadas e outras propriedades}

Ao longo do trabalho, em vários momentos nos questionamos sobre quais seriam generalizações consistentes. De um modo geral, a resposta sempre está ligada à preservação de características e propriedades do objeto original. No caso da $\lambda$ TF aqui apresentada, já apontou-se que ela, a exemplo da TF original, pertence à família das transformadas integrais, já que é uma transformação linear. Além disso, a $\lambda$-TF possui outras propriedades inerentes a uma TF (apresentadas na Sec. 4.1), como a translação de fase (ou simplesmente translação). Levando-se em conta que as Eqs. (4.20) e (4.26) simplesmente expressam a relação entre $\lambda$-TF e 0-TF, podese apresentar uma fórmula para expressar translações generalizadas. Seja $g(z)=$ $f[t(z)]$. A transformada de Fourier na variável $z$ satisfaz $\mathcal{F}_{0}[g(z+a)]=\mathrm{e}^{-i \omega a} \mathcal{F}_{0}[g(z)]$. Na variável $t$, esta expressão é equivalente a $\mathcal{F}_{\lambda}\left[f\left(t+\tau^{\prime}\right)\right]=\mathrm{e}^{-i \omega a} \mathcal{F}_{\lambda}[f(t)]$ para um parâmetro de translação $\tau^{\prime}$ desconhecido. Comparando as duas expressões e usando o mapeamento conforme da Eq. (4.22), conclui-se que $\lambda a=\ln \left[1+\lambda \tau^{\prime} /(1+\lambda t)\right]$. A suposição de que $a$ não deve depender de $t$ implica em $\tau^{\prime} \equiv \tau(1+\lambda t)$, onde $\tau$ é constante que satisfaz $\mathrm{e}^{a}=\mathrm{e}_{\lambda}(\tau)$. Portanto, a translação generalizada torna-se

$$
\mathcal{F}_{\lambda}\left[f\left(t \oplus_{\lambda} \tau\right)\right]=\left[\mathrm{e}_{\lambda}(\tau)\right]^{-i \omega} \mathcal{F}_{\lambda}[f(t)]
$$

Utilizando a Eq. (4.32), pode-se expressar a invariância generalizada da translação se $\left[\mathrm{e}_{\lambda}\left(\tau_{m}\right)\right]^{-i \omega}=1$, onde $\tau_{m}$ é o $m$-ésimo $\lambda$-período:

$$
\tau_{m}=\frac{\mathrm{e}^{2 \pi m \lambda / \omega}-1}{\lambda}=\ln _{\lambda}\left(\mathrm{e}^{2 \pi m / \omega}\right) .
$$

Novamente, $\tau_{1}=2 \pi / \omega$ recupera o resultado clássico, para $\lambda=0$. Para $\lambda \neq 0$, no entanto, a Eq. (4.33) exibe espaçamento não uniforme entre valores consecutivos de 
m. Como resultado, as seguintes expressões são obtidas:

$$
\begin{aligned}
& \tau_{m}+\tau_{n}=\ln _{\lambda}\left(\mathrm{e}^{2 \pi m / \omega} \otimes_{\lambda} \mathrm{e}^{2 \pi n / \omega}\right) \\
& \tau_{m}-\tau_{n}=\mathrm{e}^{2 \pi n \lambda / \omega} \ln _{\lambda} \mathrm{e}^{2 \pi(m-n) / \omega} .
\end{aligned}
$$

Na sequência, apresenta-se um resultado muito interessante, obtido ao se calcular a $\lambda$-TF de um sinal log-periódico.

\subsubsection{A $\lambda$-TF de um sinal log-periódico}

Como uma aplicação das Eqs. (4.34a) e (4.34b), pode-se usar intervalos periódicos diretamente dos dados para estimar o valor $\lambda$. Por exemplo, considere o sinal de variação lenta, um sinal log-periódico

$$
f(t)=\cos \left[\omega_{0} \ln (1+t)\right]
$$

com $t \geqslant 0$. Para $t$ próximo de zero, o período pode ser aproximado por $\tau_{\text {pequeno }} \approx$ $2 \pi / \omega_{0}$. Em geral, a diferença entre duas oscilações consecutivas é $\tau_{n+1}-\tau_{n}=$ $\mathrm{e}^{2 \pi \omega_{0} n}\left(\mathrm{e}^{2 \pi \omega_{0}}-1\right)$, ajustando $\lambda=1$ de acordo com a Eq. (4.34b). Isso permite reescrever a Eq. (4.35) como uma soma de duas funções exponenciais generalizadas:

$$
f(t)=\frac{\left[\mathrm{e}_{1}(t)\right]^{i \omega_{0}}+\left[\mathrm{e}_{1}(t)\right]^{-i \omega_{0}}}{2} .
$$

Devido ao comportamento log-periódico, a TF usual falha ao transmitir um espectro de potência que destaca a significância de $\omega_{0}$ para o sinal. De fato, o espectro de potência compreende uma distribuição de frequência na qual a maioria das contribuições vem de $|\omega|<\left|\omega_{0}\right|$, à medida que o intervalo de tempo entre oscilações consecutivas se torna cada vez maior. Por outro lado, a $\lambda$-TF captura o comportamento correto,

$$
\sqrt{\pi} \mathcal{F}_{1}\left\{\cos \left[\omega_{0} \ln (1+t)\right]\right\}=\delta\left(\omega-\omega_{0}\right)+\delta\left(\omega+\omega_{0}\right)
$$

Outras propriedades da TF original são válidas neste contexto generalizado. Isso ocorre principalmente quando a propriedade é inerente à função a ser transformada, já que utiliza-se na transformação o produto convencional entre o núcleo e $f(t)$. Além disso, este núcleo traz o fator $i \omega$ como potência e não como parte do argumento, a exemplo de outras tentativas de generalização. Na sequência, outras propriedades que se estendem da TF para a $\lambda$-TF. 


\subsubsection{Conjugação}

A propriedade da conjugação garante que, dado $f(t)$, uma função real com sua TF $\mathcal{F}[f(t)]=g(\omega)$, então $\overline{\mathcal{F}[f(t)]}=g(-\omega)$. Levando-se em conta que, se $f(t)$ é real, então $\overline{f(t)}=f(t)$, tem-se, para $\lambda$-TF:

$$
\begin{aligned}
& \overline{\mathcal{F}_{\lambda}[f(t)]}=\overline{\frac{1}{\sqrt{4 \pi}} \int_{-\infty}^{\infty} \frac{\left[\mathrm{e}_{\lambda}(t)\right]^{i \omega}}{|1+\lambda t|} f(t) d t}= \\
& =\frac{1}{\sqrt{4 \pi}} \int_{-\infty}^{\infty} \frac{\overline{\left[\mathrm{e}_{\lambda}(t)\right]^{i \omega}}}{|1+\lambda t|} f(t) d t .
\end{aligned}
$$

Neste ponto faz-se necessário estudar o conjugado de $\left[\mathrm{e}_{\lambda}(t)\right]^{i \omega}$. Considerando a definição apresentada na Sec. 2.6 do Cap. 2, em que apresenta-se

$$
\mathrm{e}_{\lambda}(t)=\lim _{\lambda^{\prime} \rightarrow \lambda}\left|1+\lambda^{\prime} t\right|^{\frac{1}{\lambda^{\prime}}} \mathrm{e}^{i \frac{\pi}{\lambda}[1-\theta(1+\lambda t)]},
$$

onde $\theta(t)$ é a função degrau, tem-se que:

$$
\begin{aligned}
\overline{\left[\mathrm{e}_{\lambda}(t)\right]^{i \omega}} & =\overline{\left\{\lim _{\lambda^{\prime} \rightarrow \lambda}\left|1+\lambda^{\prime} t\right|^{\frac{1}{\lambda^{\prime}}} \mathrm{e}^{i \frac{\pi}{\lambda}[1-\theta(1+\lambda t)]}\right\}^{i \omega}}= \\
& =\overline{\left\{\lim _{\lambda^{\prime} \rightarrow \lambda}\left|1+\lambda^{\prime} t\right|^{\frac{i \omega}{\lambda^{\prime}}}\right.} \mathrm{e}^{-\frac{\omega \pi}{\lambda}[1-\theta(1+\lambda t)]}= \\
& =\lim _{\lambda^{\prime} \rightarrow \lambda}\left|1+\lambda^{\prime} t\right|^{\frac{i \omega}{\lambda^{\prime}}} \mathrm{e}^{-\frac{\omega \pi}{\lambda}[1-\theta(1+\lambda t)]}= \\
& =\lim _{\lambda^{\prime} \rightarrow \lambda}\left|1+\lambda^{\prime} t\right|^{\frac{-i \omega}{\lambda^{\prime}}} \mathrm{e}^{-\frac{\omega \pi}{\lambda}[1-\theta(1+\lambda t)]}= \\
& =\lim _{\lambda^{\prime} \rightarrow \lambda}\left|1+\lambda^{\prime} t\right|^{\frac{-i \omega}{\lambda^{\prime}}} \frac{\mathrm{e}^{\frac{\omega \pi}{\lambda}[1-\theta(1+\lambda t)]}}{\mathrm{e}^{\frac{2 \omega \pi}{\lambda}}[1-\theta(1+\lambda t)]}= \\
& =\left\{\lim _{\lambda^{\prime} \rightarrow \lambda}\left|1+\lambda^{\prime} t\right|^{\frac{1}{\lambda^{\prime}}}\right\}^{-i \omega} \frac{\left\{\mathrm{e}^{i \frac{\pi}{\lambda}[1-\theta(1+\lambda t)]}\right\}^{-i \omega}}{\mathrm{e}^{\frac{2 \omega \pi}{\lambda}[1-\theta(1+\lambda t)]}}= \\
& =\frac{1}{\mathrm{e}^{\frac{2 \omega \pi}{\lambda}[1-\theta(1+\lambda t)]}\left[\mathrm{e}_{\lambda}(t)\right]^{-i \omega}} .
\end{aligned}
$$

Se $\lambda \rightarrow 0$ retomamos o resultado $\overline{\left[\mathrm{e}_{0}(t)\right]^{i \omega}}=\mathrm{e}^{-i \omega t}$. Substituindo esse resultado na Eq. (4.38), obtém-se que

$$
\begin{aligned}
\overline{\mathcal{F}_{\lambda}[f(t)]} & =\frac{1}{\sqrt{4 \pi}} \int_{-\infty}^{\infty} \frac{\overline{\left[\mathrm{e}_{\lambda}(t)\right]^{i \omega}}}{|1+\lambda t|} f(t) d t= \\
& =\frac{1}{\sqrt{4 \pi}} \int_{-\infty}^{\infty} \frac{\left[\mathrm{e}_{\lambda}(t)\right]^{-i \omega}}{|1+\lambda t|}\left[\frac{f(t)}{\left.\mathrm{e}^{\frac{2 \omega \pi[1-\theta(1+\lambda t)]}{\lambda}}\right] d t=}\right. \\
& =\frac{1}{\sqrt{4 \pi}}\left\{\mathrm{e}^{\frac{-2 \pi \omega}{\lambda}} \int_{-\infty}^{-1 / \lambda} \frac{\left[\mathrm{e}_{\lambda}(t)\right]^{-i \omega}}{|1+\lambda t|} f(t) d t+\int_{-1 / \lambda}^{\infty} \frac{\left[\mathrm{e}_{\lambda}(t)\right]^{-i \omega}}{|1+\lambda t|} f(t) d t\right\} .
\end{aligned}
$$


A Eq. (4.41) retoma a validade da propriedade da conjugação da TF original quando $\lambda \rightarrow 0$. Para obter um resultado mais expressivo, basta considerar apenas o intervalo em que $e_{\lambda}(t)$ é real. Neste caso, tem-se que

$$
\overline{\left[\mathrm{e}_{\lambda}(t)\right]^{i \omega}}=\left[\mathrm{e}_{\lambda}(t)\right]^{-i \omega}
$$

e, desta forma,

$$
\begin{aligned}
\overline{\mathcal{F}_{\lambda}[f(t)]} & =\frac{1}{\sqrt{4 \pi}} \int_{-\infty}^{\infty} \frac{\overline{\left[\mathrm{e}_{\lambda}(t)\right]^{i \omega}}}{|1+\lambda t|} f(t) d t= \\
& =\frac{1}{\sqrt{4 \pi}} \int_{-\infty}^{\infty} \frac{\left[\mathrm{e}_{\lambda}(t)\right]^{-i \omega}}{|1+\lambda t|} f(t) d t \equiv \\
& \equiv \frac{1}{\sqrt{4 \pi}} \int_{-\infty}^{\infty} \mathrm{e}^{-i \omega z} f\left(t_{z}\right) d z+\frac{\mathrm{e}^{-\pi \frac{\omega}{\lambda}}}{\sqrt{4 \pi}} \int_{-\infty}^{\infty} \mathrm{e}^{-i \omega z} f\left(t_{z}\right) d z= \\
& =\left(\frac{1+\mathrm{e}^{-\frac{\pi \omega}{\lambda}}}{\sqrt{4 \pi}}\right) \int_{-\infty}^{\infty} \mathrm{e}^{-i \omega z} f\left(t_{z}\right) d z,
\end{aligned}
$$

$\operatorname{com} t_{z}=\ln _{\lambda}\left(\mathrm{e}^{z}\right)$. Na Eq. (4.43), quando $\lambda \rightarrow 0$, retoma-se a validade da propriedade para a TF usual.

\subsection{3 $\lambda$-TF da derivada}

A Transformada da derivada de uma função $f(t)$ em que $\lim _{t \rightarrow \infty} f(t)=0$, possui a seguinte propriedade:

$$
\mathcal{F}\left[f^{\prime}(t)\right]=i \omega \mathcal{F}[f(t)]
$$

Ao expandir a TF para $\lambda$-TF mantém-se essa propriedade, já que, utilizando o mapeamento conforme, $z=\ln \left[\mathrm{e}_{\lambda}(t)\right]$ e integração por partes, tem-se que

$$
\begin{aligned}
\mathcal{F}_{\lambda}\left[f^{\prime}(t)\right] & =\frac{1}{\sqrt{4 \pi}} \int_{-\infty}^{\infty} \frac{\left[\mathrm{e}_{\lambda}(t)\right]^{i \omega}}{|1+\lambda t|} f^{\prime}(t) d t= \\
& =\left(\frac{1+\mathrm{e}^{-\pi \omega / \lambda}}{\sqrt{4 \pi}}\right)\left[\lim _{R \rightarrow \infty} \int_{-R}^{R} \mathrm{e}^{i \omega z} f^{\prime}\left(t_{z}\right) d z\right]= \\
& =\left(\frac{1+\mathrm{e}^{-\pi \omega / \lambda}}{\sqrt{4 \pi}}\right)\left\{\lim _{R \rightarrow \infty}\left(\left[\mathrm{e}^{i \omega z} f\left(t_{z}\right)\right]_{-R}^{R}-\int_{-R}^{R} i \omega \mathrm{e}^{i \omega z} f\left(t_{z}\right) d z\right)\right\}= \\
& =\left(\frac{1+\mathrm{e}^{-\pi \omega / \lambda}}{\sqrt{4 \pi}}\right)(-i \omega) \int_{-\infty}^{\infty} \mathrm{e}^{i \omega z} f\left(t_{z}\right)= \\
& =-i \omega\left(1+\mathrm{e}^{-\pi \omega / \lambda}\right) \mathcal{F}[f(t)],
\end{aligned}
$$


que para $\lambda=0$ retoma a validade da Eq. (4.44). É importante notar que $t_{z}=\ln _{\lambda}\left(\mathrm{e}^{z}\right)$ e, dessa forma, $\lim _{R \rightarrow \infty}\left[\mathrm{e}^{i \omega z} f\left(t_{z}\right)\right]_{-R}^{R}=0$, já que

$$
\begin{aligned}
\lim _{R \rightarrow \infty}\left[\mathrm{e}^{i \omega z} f\left(t_{z}\right)\right]_{-R}^{R} & =\lim _{R \rightarrow \infty}\left[\mathrm{e}^{i \omega R} f\left(\frac{\mathrm{e}^{R \lambda}-1}{\lambda}\right)-\mathrm{e}^{-i \omega R} f\left(\frac{\mathrm{e}^{-R \lambda}-1}{\lambda}\right)\right]= \\
& =\lim _{R \rightarrow \infty} \mathrm{e}^{i \omega R} f\left(\frac{\mathrm{e}^{R \lambda}-1}{\lambda}\right)=0,
\end{aligned}
$$

pois $\lim _{R \rightarrow \infty}\left(\mathrm{e}^{R \lambda}-1\right) / \lambda=\infty$ e $\lim _{t \rightarrow \infty} f(t)=0$.

\subsubsection{Transformada da Integral}

De acordo com a Eq. (4.8), dada uma função $f(t)$ e sua respectiva TF, $\mathcal{F}[f(t)]$, de tal forma que $\mathcal{F}[0]=0$, então, $\mathcal{F}\left[\int_{-\infty}^{t} f(\tau) d \tau\right]=\mathcal{F}[f(t)] / i \omega$. É possível estender essa propriedade para $\lambda$-TF. Para isso, basta considerar uma função $g(t)$ de tal forma que

$$
g(t)=\int_{-\infty}^{t} f(\tau) d \tau
$$

Pelo teorema fundamental do cálculo temos que $g^{\prime}(t)=f(t)$ e, desta forma

$$
\mathcal{F}_{\lambda}\left[g^{\prime}(t)\right]=\mathcal{F}_{\lambda}[f(t)]
$$

Além disso, se $\mathcal{F}_{\lambda}[0]=0$, tem-se que

$$
\lim _{t \rightarrow \infty} g(t)=\int_{-\infty}^{\infty} f(\tau) d \tau=\int_{-\infty}^{\infty} f(\tau) \mathrm{e}^{i 0 \tau} d \tau=\mathcal{F}_{\lambda}[0]=0,
$$

como também,

$$
\lim _{t \rightarrow-\infty} g(t)=\int_{-\infty}^{-\infty} f(\tau) d \tau=0
$$

As equações Eq. (4.49) e Eq. (4.50) abrem possibilidade de uso da propriedade da Transformada da derivada apresentada na Eq. (4.45) e, assim,

$$
\mathcal{F}_{\lambda}\left[g^{\prime}(t)\right]=-i \omega\left(1+\mathrm{e}^{-\pi \omega / \lambda}\right) \mathcal{F}_{\lambda}[g(t)]
$$

que, por conseguinte,

$$
\mathcal{F}_{\lambda}[f(t)]=-i \omega\left(1+\mathrm{e}^{-\pi \omega / \lambda}\right) \mathcal{F}_{\lambda}\left[\int_{-\infty}^{t} f(\tau) d \tau\right] .
$$

Desta forma, chega-se a uma versão generalizada da propriedade da Transformada da Integral, a saber

$$
\mathcal{F}_{\lambda}\left[\int_{-\infty}^{t} f(\tau) d \tau\right]=\frac{i}{\omega\left(1+\mathrm{e}^{-\pi \omega / \lambda}\right)} \mathcal{F}_{\lambda}[f(t)]
$$




\subsubsection{Modulação}

Outra propriedade da TF usual é a modulação, apresentada na Eq. (4.9). Sabe-se que, na versão original, dados $f(t)$ e sua $\mathrm{TF} \mathcal{F}[f(t)]=F(\omega)$, então $\mathcal{F}\left[f(t) \cos \left(\omega_{0}, t\right)\right]=\left[F\left(\omega+\omega_{0}\right)+F\left(\omega-\omega_{0}\right)\right] / 2$. É possível apresentar uma versão generalizada desta propriedade. Para isso será necessário utilizar uma nova generalização da função cosseno, a qual será discutida com maior profundidade no Cap. 5. Este cosseno generalizado $c_{\lambda}$, é definido por

$$
\mathrm{c}_{\lambda}\left(\omega_{0}, t\right)=\frac{\left[\mathrm{e}_{\lambda}(t)\right]^{i \omega_{0}}+\left[\mathrm{e}_{\lambda}(t)\right]^{-i \omega_{0}}}{2} .
$$

Assim, dada uma função $f(t)$, e sua respectiva $\lambda$-TF, $\mathcal{F}_{\lambda}[f(t)]=F_{\lambda}(\omega)$, conforme a Eq. (4.20), tem-se que:

$$
\begin{aligned}
\mathcal{F}\left[f(t) \mathrm{c}_{\lambda}\left(\omega_{0}, t\right)\right] & =\frac{1}{\sqrt{4 \pi}} \int_{-\infty}^{\infty} \frac{\left[\mathrm{e}_{\lambda}(t)\right]^{i \omega}}{|1+\lambda t|} f(t) \mathrm{c}_{\lambda}\left(\omega_{0}, t\right)= \\
& =\frac{1}{\sqrt{4 \pi}} \int_{-\infty}^{\infty} \frac{\left[\mathrm{e}_{\lambda}(t)\right]^{i \omega}}{|1+\lambda t|} f(t)\left\{\frac{\left[\mathrm{e}_{\lambda}(t)\right]^{i \omega_{0}}+\left[\mathrm{e}_{\lambda}(t)\right]^{-i \omega_{0}}}{2}\right\}= \\
& =\frac{1}{\sqrt{4 \pi}}\left\{\int_{-\infty}^{\infty} \frac{1}{2} \frac{\left[\mathrm{e}_{\lambda}(t)\right]^{i\left(\omega+\omega_{0}\right)}}{|1+\lambda t|} f(t)+\int_{-\infty}^{\infty} \frac{1}{2} \frac{\left[\mathrm{e}_{\lambda}(t)\right]^{i\left(\omega-\omega_{0}\right)}}{|1+\lambda t|} f(t)\right\}= \\
& =\frac{1}{2}\left\{\frac{1}{\sqrt{4 \pi}} \int_{-\infty}^{\infty} \frac{\left[\mathrm{e}_{\lambda}(t)\right]^{i\left(\omega+\omega_{0}\right)}}{|1+\lambda t|} f(t)+\frac{1}{\sqrt{4 \pi}} \int_{-\infty}^{\infty} \frac{\left[\mathrm{e}_{\lambda}(t)\right]^{i\left(\omega-\omega_{0}\right)}}{|1+\lambda t|} f(t)\right\}= \\
& =\frac{1}{2}\left\{F_{\lambda}\left(\omega+\omega_{0}\right)+F_{\lambda}\left(\omega-\omega_{0}\right)\right\} .
\end{aligned}
$$

Quando $\lambda \rightarrow 0$ retoma-se a propriedade de modulação da TF usual.

\subsection{Inverso}

Outra importante característica da TF usual é possuir inversa. Já foi apontado que algumas generalizações anteriores não possuem uma inversa adequada. A Eq. (4.20) não dá pistas sobre como encontrar a transformação inversa. A mudança de $i$ para $-i$ na função exponencial generalizada também fornece pouca ajuda nessa tarefa. Ao invés de criar suposições em torno da relação entre $\mathcal{F}_{\lambda}[f]$ e 0 -TF na Eq. (4.26), pode-se definir adequadamente o inverso. Mais especificamente, este resultado já é conhecido na coordenada mapeada $z$, pois é a TF usual. Portanto, é suficiente mapear a 0-TF inversa para a coordenada original $t$ por meio de $\tilde{g}(\omega)$. Aplicando a Eq. (4.22) a $\int_{-\infty}^{\infty} \tilde{g}(\omega) \mathrm{e}^{i \omega z} d z$, mostra-se que o mapa afeta apenas a função exponencial, $\mathrm{e}^{i \omega z}=\left[\mathrm{e}_{\lambda}(t)\right]^{-i \omega}$. Assim, o seguinte inverso generalizado é proposto: 


$$
\mathcal{F}_{\lambda}^{-1}[\tilde{g}(t)]=\frac{1}{\sqrt{4 \pi}} \int_{-\infty}^{\infty}\left[\mathrm{e}_{\lambda}(t)\right]^{-i \omega} \tilde{g}(\omega) d \omega .
$$

Apesar da impressionante falta de simetria entre as Eqs. (4.20) e (4.56), devido à ausência de $|1+\lambda t|^{-1}$, ambos produzem 0-TF simétrico na coordenada conforme $z$.

Como exemplo, observe $\mathcal{F}_{\lambda}[1]=\sqrt{4 \pi} \delta(\omega)$ então,

$$
\mathcal{F}_{\lambda}^{-1}\left[\mathcal{F}_{\lambda}[1]\right]=\frac{1}{\sqrt{4 \pi}} \int_{-\infty}^{\infty} \sqrt{4 \pi} \delta(\omega) \mathrm{e}_{\lambda}(t)^{-i \omega} d \omega=1
$$

como esperado. Alternativamente, pode-se também inverter a ordem de integração para calcular a integral:

$$
\begin{aligned}
\mathcal{F}_{\lambda}^{-1}\left[\mathcal{F}_{\lambda}[1]\right] & =\frac{1}{\sqrt{4 \pi}} \int_{-\infty}^{\infty}\left[\mathrm{e}_{\lambda}(t)\right]^{i \omega} d \omega \int_{-\infty}^{\infty} \frac{\left[\mathrm{e}_{\lambda}\left(t^{\prime}\right)\right]^{i \omega}}{\left|1+\lambda t^{\prime}\right|} \\
& =\frac{1}{\sqrt{4 \pi}} \int_{-\infty}^{\infty} d x^{\prime}\left[\int_{-\infty}^{\infty} \mathrm{e}^{i \omega\left(x^{\prime}-x\right)} d \omega+\lim _{R \rightarrow \infty} \int_{-\infty}^{\infty} \mathrm{e}^{i \omega\left(x^{\prime}-x\right)} \mathrm{e}^{-\pi \omega / \lambda} d \omega\right] \\
& =\frac{1}{2}-\lim _{R \rightarrow \infty} \frac{\mathrm{e}^{\pi R / \lambda}}{4 i \pi} \int_{-\infty}^{\infty} \frac{\mathrm{e}^{-i R\left(x^{\prime}-x\right)} d x^{\prime}}{\left(x^{\prime}-x\right)+i \pi / \lambda}=1
\end{aligned}
$$

onde a última integral está associada a uma integral de contorno fechada que envolve o polo em $z=-i \pi / \lambda$. O mesmo argumento também pode ser estendido a outras funções analíticas. Por uma questão de clareza, assuma $t>t_{c}$ para que

$$
\mathcal{F}_{\lambda}^{-1}\left[\mathcal{F}_{\lambda}[f]\right]=\frac{f(t)}{2}-\lim _{R \rightarrow \infty} \frac{\mathrm{e}^{\pi R / \lambda}}{4 i \pi} \int_{-\infty}^{\infty} \frac{\left.\mathrm{e}^{-i R\left[x^{\prime}-z(t)\right]} f\left[t\left(x^{\prime}+i \pi / \lambda\right)\right] d x^{\prime}\right)}{x^{\prime}-z(t)+i \pi / \lambda}=f(t) .
$$

Para funções mais gerais, é preciso avaliar se a ordem de integração pode ser trocada.

\subsection{Conclusão}

Funções generalizadas encontram aplicações em vários campos de pesquisa, com ênfase especial na análise de dados. Ao usar transformações não lineares com um único grau de liberdade, geralmente se tenta simplificar a distribuição de dados ou a extração de recursos destes dados. A $\lambda$-TF, apresentada por Umarov e Tsallis [63] na Eq. (4.15), pertence a este último grupo. A vantagem daquela proposta é se adequar bem à manipulação de Gaussianas generalizadas sobre uma classe mais ampla de funções. Em nossa proposta, impomos linearidade para estender o conceito de transformadas de Fourier generalizadas e, desta forma, conseguimos famílias de funções que pertencem à classe das transformadas integrais. Essa nova expressão para a $\lambda$-transformada de Fourier estendida na Eq. (4.20) baseia-se inteiramente 
no mapeamento conforme descrito na Eq. (4.22), tendo a representação usual da transformada de Fourier no plano complexo. Esse mapeamento, aliás, segue as mesmas diretrizes da transformação de Box-Cox, porém ao invés de transformar os dados, transforma o procedimento. Além disso, nossas descobertas fornecem uma representação fiel da distribuição delta de Dirac, abrindo inclusive a possibilidade de escrevê-la em termos da $\lambda$-TF. Introduz o conceito de $\lambda$-períodos generalizados, valida e generaliza outras importantes propriedades da TF usual e apresenta alguns exemplos. Nesse contexto, é possível definir um novo conjunto de funções trigonométricas generalizadas, compatíveis com os $\lambda$-períodos generalizados, que recuperam as propriedades trigonométricas usuais, conforme é apresentado no Cap. 5. O próximo capítulo traz, ainda, a generalização das funções log-periódicas. Tais funções foram utilizadas em vários exemplos no decorrer deste capítulo. 


\title{
5
}

\section{Funções Log-periódicas Generalizadas}

\author{
Faça as coisas o mais simples que você puder, porém não se restrinja \\ às mais simples. \\ - Albert Einstein
}

Log-periodicidade, ou periodicidade logarítmica, é um termo utilizado na Física desde a década de 1960 [73]. Contudo, foi a partir dos anos 90 que esta expressão ganhou força e notoriedade. Os estudos, quase simultâneos, de Feigenbaum e Freund [25] e de Didier Sornette [24] acerca das oscilações no mercado financeiro abriram espaço para importantes discussões e descobertas relativas a esse tema. Neste capítulo, apresenta-se uma relação entre funções log-periódicas e funções logaritmo e exponenciais generalizadas. Propõe-se, ainda, novas generalizações para as funções trigonométricas e hiperbólicas.

\subsection{Funções periódicas}

Eventos periódicos são observáveis em nosso quotidiano. Ao esperar por um trem com linha regular, ao observar o movimento das marés, as estações do ano, entre outros fenômenos, percebe-se a repetição de algum evento após um determinado período. Funções que modelam eventos periódicos são denominadas funções periódicas. Diz-se que uma função $f: \mathbb{R} \rightarrow \mathbb{R}$ é periódica se, dada uma constante real $k \neq 0$ e $n \in \mathbb{Z}$, então,

$$
f(x+n k)=f(x), \forall x \in \mathbb{R}
$$


Existe, ainda, uma extensão deste conceito para o plano complexo: as funções duplamente periódicas (funções meromorfas). Neste caso, têm-se que $f\left(x+k_{1}\right)=$ $f\left(x+k_{2}\right)=f(x)$, com $k_{1}, k_{2} \in \mathbb{C}$ e $k_{1} / k_{2} \notin \mathbb{R}$. São exemplos de funções periódicas reais a função constante e as funções trigonométricas. Essas últimas são amplamente estudadas por suas características, diversas propriedades e, principalmente, por suas inúmeras aplicações. Na sequência, uma breve discussão sobre essas funções.

\subsubsection{Funções trigonométricas}

Os primeiros indícios da trigonometria surgiram no Egito e na Babilônia por volta do ano 1650 a.C [36] e ganhou status de ciência com o astrônomo grego Hiparco de Niceia (190 a.C. a 125 a.C.). Já na era contemporânea, por volta de 1710, com Thomas-Fanten de Lagny, foi aprofundada por estudos relacionados a funções trigonométricas, os quais confirmaram o seu caráter periódico. Com o surgimento do cálculo diferencial, se consolidou como importante área da matemática, com aplicações nas mais variadas ciências, tais como Medicina, Física, Química, Engenharia e Astronomia.

As funções trigonométricas e a introdução do radiano são consideradas um avanço da trigonometria primitiva, já que saem do campo observável (relações entre ângulos e lados de um triângulo real) e caminham para o campo abstrato. Desta forma, propriedades válidas para as razões valem, também, para as funções. As principais funções trigonométricas são: cosseno, seno e tangente, sendo que a terceira é a razão entre a segunda e a primeira. Existem, ainda, as relações secundárias: secante, cossecante e cotangente, que surgem do inverso multiplicativo das funções principais. Desta forma, pode-se dizer que, à partir do cosseno e do seno, podese obter as demais funções mediante manipulações algébricas adequadas. Assim, limita-se este trabalho a generalização dessas duas funções, já que, tudo o que for apresentado aqui pode ser estendido às demais funções de maneira relativamente simples.

As funções cosseno e seno tiveram um tratamento analítico à partir da obra "Introductio in Analysin Infinitorum" de Leonhard Euler, de 1748. Nela, Euler apresenta uma definição para tais funções a partir da função exponencial, a saber:

$$
\cos (\omega t)=\frac{\mathrm{e}^{i \omega t}+\mathrm{e}^{-i \omega t}}{2}
$$




$$
\operatorname{sen}(\omega t)=\frac{\mathrm{e}^{i \omega t}-\mathrm{e}^{-i \omega t}}{2 i}
$$

em que $i=\sqrt{-1}$ é a unidade imaginária e $\omega$ é uma constante chamada de frequência angular. Estas funções são definidas para todo número real e possuem diversas características e propriedades das quais destacam-se:

1. Funções periódicas e limitadas: em suas versões mais simples, é possível verificar que tanto a função seno, $\operatorname{sen}(t)$, quanto a cosseno, $\cos (t)$, são periódicas (período igual a $2 \pi$ ) e limitadas ao intervalo $-1 \leq f(t) \leq 1$.

2. Paridade da função $\cos (t)$ : para qualquer $t \in \mathbb{R}$ tem-se que:

$$
\cos (-\omega t)=\cos (\omega t)
$$

3. Imparidade da função sen $(t)$ : para qualquer $t \in \mathbb{R}$ tem-se que:

$$
\operatorname{sen}(-\omega t)=-\operatorname{sen}(\omega t)
$$

4. Relação fundamental da trigonometria: para qualquer $t \in \mathbb{R}$ vale a igualdade:

$$
\cos ^{2}(\omega t)+\operatorname{sen}^{2}(\omega t)=1
$$

5. Relação de Euler: dados $\omega$, constante real, e a variável $t \in \mathbb{R}$, é válida a seguinte relação:

$$
\mathrm{e}^{ \pm i \omega t}=\cos (\omega t) \pm i \sin (\omega t)
$$

6. Fórmula de De Moivre: dados $\omega$, constante real, e a variável $t \in \mathbb{R}$, então:

$$
[\cos (\omega t) \pm i \operatorname{sen}(\omega t)]^{n}=\cos (n \omega t) \pm i \operatorname{sen}(n \omega t)
$$

7. Soma de Arcos: dados $a, b \in \mathbb{R}$ valem as seguintes relações:

$$
\begin{aligned}
\cos [\omega(a \pm b)] & =\cos (\omega a) \cos (\omega b) \mp \operatorname{sen}(\omega a) \operatorname{sen}(\omega b) \\
\operatorname{sen}[\omega(a \pm b)] & =\operatorname{sen}(\omega a) \cos (\omega b) \pm \operatorname{sen}(\omega b) \cos (\omega a)
\end{aligned}
$$

8. Transformação de produto em soma: dados $a, b \in \mathbb{R}$ valem as seguintes transformações:

$$
\begin{aligned}
\operatorname{sen}(\omega a) \cos (\omega b) & =\frac{1}{2}\{\operatorname{sen}[\omega(a+b)]+\operatorname{sen}[\omega(a-b)]\} \\
\operatorname{sen}(\omega a) \operatorname{sen}(\omega b) & =\frac{1}{2}\{\cos [\omega(a-b)]-\cos [\omega(a+b)]\} \\
\cos (\omega a) \cos (\omega b) & =\frac{1}{2}\{\cos [\omega(a+b)]+\cos [\omega(a-b)]\}
\end{aligned}
$$


No campo do cálculo diferencial e integral, existem alguns resultados importantes relacionados a estas funções, que devem ter o devido destaque. O primeiro é chamado de limite fundamental da função seno, dado por

$$
\lim _{t \rightarrow 0} \frac{\operatorname{sen}(\omega t)}{t}=\omega
$$

Esse resultado é muito importante para o cálculo de modo geral, pois ele ajuda simplificar limites de expressões mais complicadas que envolvam funções trigonométricas.

Quanto às derivadas de ordem $n$ das funções cosseno e seno, pode-se observar que elas são cíclicas e se repetem a cada 4 novas derivações. Desta forma, têm-se como derivadas das funções $\cos (\omega t)$ e $\operatorname{sen}(\omega t)$, respectivamente:

$$
\begin{aligned}
& \frac{d^{n}}{d t^{n}} \cos (\omega t)=\omega^{n} T_{n+1}(\omega t) \\
& \frac{d^{n}}{d t^{n}} \operatorname{sen}(\omega t)=\omega^{n} T_{n}(\omega t),
\end{aligned}
$$

em que

$$
T_{k}(\omega t)=\left\{\begin{array}{rrr}
-\operatorname{sen}(\omega t) & \text { se } & \bmod (k, 4)=1, \\
-\cos (\omega t) & \text { se } & \bmod (k, 4)=2, \\
\operatorname{sen}(\omega t) & \text { se } & \bmod (k, 4)=3, \\
\cos (\omega t) & \text { se } & \bmod (k, 4)=0 .
\end{array}\right.
$$

A expressão $\bmod (k, 4)$ indica o resto da divisão de $k$ por 4 .

Por outro lado, verifica-se que as funções trigonométricas tradicionais são de fácil integração, já que:

$$
\begin{aligned}
& \int \cos (\omega t) d t=\frac{\operatorname{sen}(\omega t)}{\omega}+C_{1} \\
& \int \operatorname{sen}(\omega t) d t=-\frac{\cos (\omega t)}{\omega}+C_{2},
\end{aligned}
$$

com $C_{1}$ e $C_{2}$ constantes de integração. Acrescenta-se, ainda, que funções trigonométricas são de fundamental importância para resolução de outras integrais por meio de substituições.

Existe, ainda, uma outra categoria de funções muito importantes na física e matemática: as funções hiperbólicas, apresentadas a seguir. 


\subsubsection{Funções hiperbólicas}

As funções hiperbólicas foram introduzidas na matemática a partir de 1760 de maneira simultânea e independente por dois matemáticos: Riccati e Lambert. Existem várias curiosidades e aplicações ligadas às funções hiperbólicas. Um bom exemplo foi o desafio proposto no final do séc. XVII por Jakob Bernoulli que envolvia duas hastes e um fio suspenso: o chamado problema da catenária. O propósito deste desafio era descobrir que curva era formada por esse fio. A primeira solução correta foi apresentada por outro Bernoulli: Johann, irmão de Jakob. Outros importantes nomes da matemática também chegaram ao mesmo resultado de Johann, dentre os quais pode-se citar Gottfried Leibniz e Christiaan Huygens. Estes, de diferentes formas, chegaram a expressões relacionadas à funções hiperbólicas [74].

As funções hiperbólicas são similares às funções trigonométricas, porém, enquanto as trigonométricas se relacionam com o círculo, estas são relacionadas com hipérboles. As funções hiperbólicas básicas são: cosseno hiperbólico, $\cosh (t)$, seno hiperbólico, $\operatorname{senh}(t)$, e tangente hiperbólica, $\tanh (t)$. Esta última é obtida pela razão das duas primeiras. Já as relações secundárias são: secante hiperbólica, sech $(t)$, cossecante hiperbólica, $\operatorname{cosech}(t)$, e cotangente hiperbólica, $\operatorname{cotah}(t)$, que são obtidas, respectivamente, pelo inverso multiplicativo das funções básicas. A exemplo das funções trigonométricas, a partir de $\cosh (t)$ e $\operatorname{senh}(t)$ é possível se obter as demais funções. Desta forma, muitas propriedades relativas às outras relações podem ser obtidas mediante operações básicas, partindo de $\cosh (t)$ e $\operatorname{senh}(t)$.

As funções hiperbólicas também podem ser escritas a partir de exponenciais. Desta forma, escreve-se $\cosh (t)$ e $\operatorname{senh}(t)$ como:

$$
\begin{aligned}
\cosh (\omega t) & =\frac{\mathrm{e}^{\omega t}+\mathrm{e}^{-\omega t}}{2} ; \\
\operatorname{senh}(\omega t) & =\frac{\mathrm{e}^{\omega t}-\mathrm{e}^{-\omega t}}{2},
\end{aligned}
$$

onde $\omega$ é a frequência angular. Tanto cosseno quanto o seno hiperbólico são funções periódicas considerando a parte imaginária na variável it, pois reencontra-se as funções trigonométricas. De fato, este termo (it) acaba conectando as funções hiperbólicas com as trigonométricas, já que

$$
\operatorname{sen}(i \omega t)=i \operatorname{senh}(\omega t)
$$




$$
\cos (i \omega t)=\cosh (\omega t)
$$

ou, de outra forma,

$$
\begin{aligned}
\cos (\omega t) & =\cosh (i \omega t), \\
\operatorname{sen}(\omega t) & =i \operatorname{senh}(-i \omega t) .
\end{aligned}
$$

As funções hiperbólicas também possuem uma série de propriedades, das quais destacamos:

1. Relação da soma: $\forall t, \omega \in \mathbb{R}$,

$$
\operatorname{senh}(\omega t)+\cosh (\omega t)=\mathrm{e}^{\omega t}
$$

2. Relação fundamental: para quaisquer $t$ e $\omega$, reais, tem-se que:

$$
\cosh ^{2}(\omega t)-\operatorname{senh}^{2}(\omega t)=1
$$

3. Potência da soma: dados $t, \omega, n \in \mathbb{R}$, vale a igualdade:

$$
[\operatorname{senh}(\omega t)+\cosh (\omega t)]^{n}=\operatorname{senh}(n \omega t)+\cosh (n \omega t)
$$

4. Soma de arcos: dados $a, b, \omega \in \mathbb{R}$, valem as seguintes somas:

$$
\begin{aligned}
& \operatorname{senh}[\omega(a+b)]=\operatorname{senh}(\omega a) \cosh (\omega b)+\cosh (\omega a) \operatorname{senh}(\omega b) \\
& \cosh [\omega(a+b)]=\cosh (\omega a) \cosh (\omega b)+\operatorname{senh}(\omega a) \operatorname{senh}(\omega b)
\end{aligned}
$$

5. Propriedade do quadrado: para quaisquer $t$ e $\omega$, reais, tem-se que:

$$
\begin{aligned}
\cosh ^{2}(\omega t) & =\frac{\cosh (2 \omega t)+1}{2} ; \\
\operatorname{senh}^{2}(\omega t) & =\frac{\cosh (2 \omega t)-1}{2} .
\end{aligned}
$$

Já no cálculo diferencial, existe um limite fundamental para o seno hiperbólico, muito semelhante à Eq. (5.10), dado por

$$
\lim _{t \rightarrow 0} \frac{\operatorname{senh}(\omega t)}{t}=\omega .
$$

As derivadas destas funções lembram muito o comportamento das funções trigonométricas, já que ficam se alternando entre $\cosh (\omega t)$ e senh $(\omega t)$. A diferença é 
que nas funções hiperbólicas o ciclo é 2, ao invés de 4 . Pode-se escrever as derivadas das funções $\cosh (\omega t)$ e $\operatorname{senh}(\omega t)$ respectivamente como:

$$
\begin{aligned}
& \frac{d^{n} \cosh (\omega t)}{d t^{n}}=\omega^{n} H_{n}(\omega t) ; \\
& \frac{d^{n} \operatorname{senh}(\omega t)}{d t^{n}}=\omega^{n} H_{n+1}(\omega t),
\end{aligned}
$$

em que

$$
H_{k}(\omega t)= \begin{cases}\cosh (\omega t) & \text { se } k \text { é par } \\ \operatorname{senh}(\omega t) & \text { se } k \text { é impar }\end{cases}
$$

No que se refere a integral, as funções cosseno hiperbólico e seno hiperbólico possuem resultado muito simples, já que

$$
\begin{aligned}
& \int \cosh (\omega t) d t=\frac{1}{\omega} \operatorname{senh}(\omega t)+C_{1} ; \\
& \int \operatorname{senh}(\omega t) d t=\frac{1}{\omega} \cosh (\omega t)+C_{2},
\end{aligned}
$$

com $C_{1}$ e $C_{2}$ constantes.

Tanto as funções hiperbólicas quanto as trigonométricas podem ser escritas a partir da função exponencial e isso motivou a busca por sua generalização por meio da função exponencial generalizada $\mathrm{e}_{\lambda}(t)$ [31]. Na sequência, relaciona-se funções log-periódicas e funções exponenciais generalizadas.

\subsection{Funções log-periódicas}

Funções log-periódicas são as que oscilam de acordo com o logaritmo da variável ao invés da própria variável. Assim, $g: \mathbb{R} \rightarrow \mathbb{R}$ é log-periódica se, dado $n \in \mathbb{Z}$ e $k \in \mathbb{R}$, então

$$
g\left(x+\mathrm{e}^{n k}\right)=g(x), \forall x \in \mathbb{R} .
$$

Elas possuem como principal característica oscilar em períodos cada vez mais longos ou cada vez mais curtos, dependendo do caso estudado. A Fig. (5.1) mostra, como exemplo, o comportamento das funções $f(x)=\cos [5 \ln (x)]$ e $g(x)=\cos [5 \ln (10-x)]$. Na Fig. (5.1), é possível verificar que $f(x)$ inicia com oscilações rápidas e, aos poucos, tem seu período aumentado, fazendo com que as oscilações sejam mais lentas. Ao contrário, $g(x)$ tem oscilações lentas perto da origem e vai acelerando próximo de $x=10$. Tanto o zero em $f(x)$ quanto o $10 \mathrm{em} g(x)$ são chamados pontos de acumulação. Estes pontos são característicos de funções log-periódicas estando presentes em 


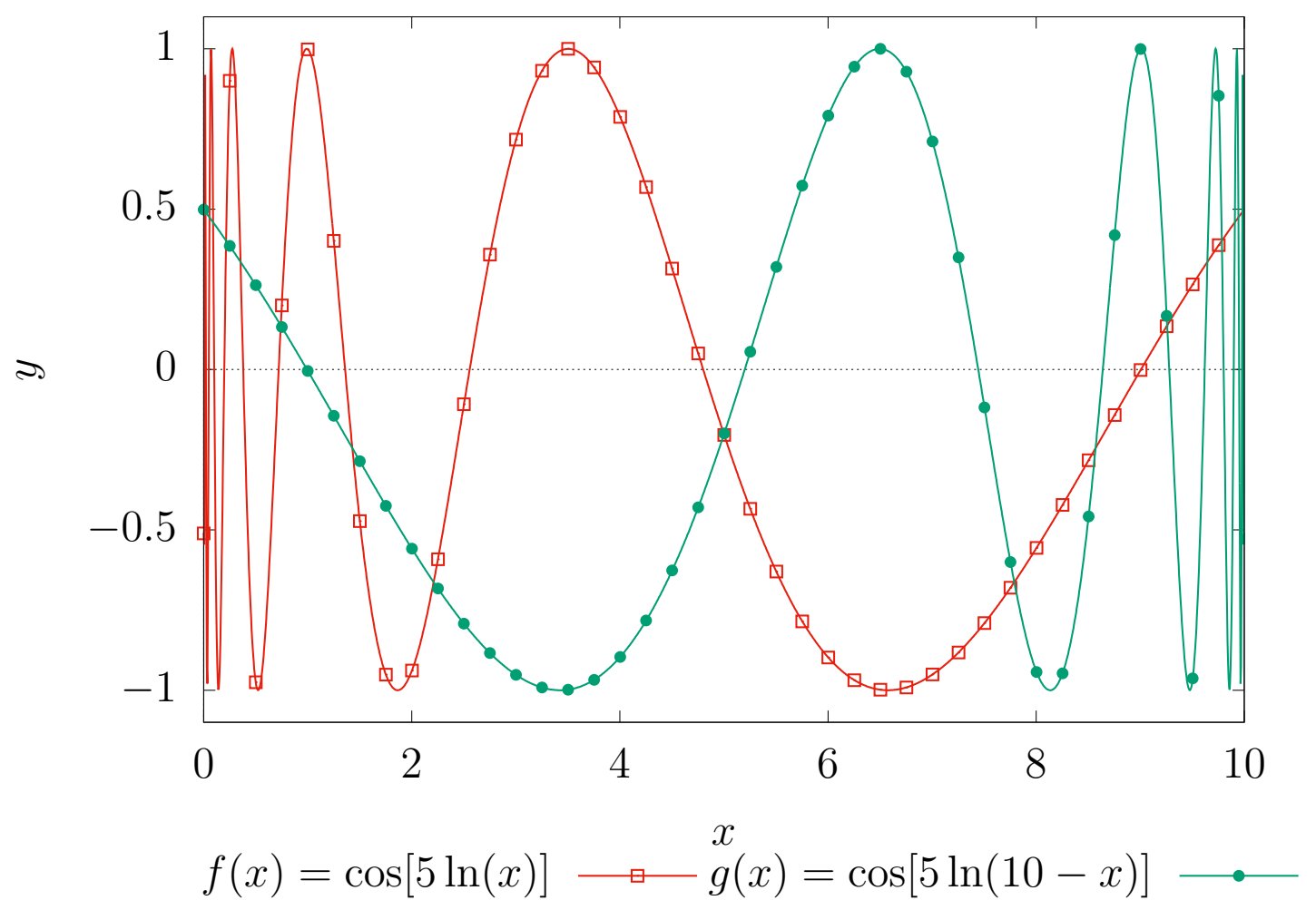

Figura 5.1: Exemplos de funções log-periódicas: $f(x)=\cos [5 \ln (x)]$ inicia oscilando rapidamente e aos poucos muda para uma oscilação mais lenta; $g(x)=\cos [5 \ln (10-x)]$ inicia oscilando lentamente e, à medida que se aproxima de 10 , tem oscilação acelerada. Aqui, o valor 10 é o ponto de acumulação.

praticamente todas as aplicações. Por exemplo, comportamentos similares ao apresentado pelas funções $f(x)$ e $g(x)$ estão presentes em estudos acerca das dinâmicas do mercado financeiro $[24,28,72,75,76]$, em que os pontos de acumulação indicam um crash (ou outra anomalia) na bolsa de valores. São outros exemplos de aplicações para funções log-periódicas a previsão de terremotos de grande magnitude [26-28], anatomia do corpo humano [77], confecção de antenas log-periódicas [73, 78, 79], entre outros $[80,81]$. As Refs. [29, 30] merecem destaque já que relacionam funções log-periódicas à função exponencial generalizada com o parâmetro $\lambda \in \mathbb{C}$ e apresenta interessantes aplicações neste sentido. Em todos os exemplos indicados, entender e localizar os pontos de acumulação são fundamentais para eficácia dos modelos.

Uma interessante observação acerca dessas funções pode ser feita analisando uma parte do núcleo da $\lambda$-TF, apresentada tanto no Cap. 4, quanto na Ref. [23]. A partir desta expressão, é possível relacionar as funções log-periódicas com as 
exponenciais generalizadas. Para tanto, apresenta-se na sequência um breve resumo das funções cosseno e seno generalizados.

\subsubsection{Funções trigonométricas e hiperbólicas generalizadas}

As funções trigonométricas generalizadas foram apresentadas por Borges [31] no final da década de 1990, partindo da função e ${ }_{\lambda}(t)$ de Tsallis. O trabalho de Borges generalizou as funções cosseno, $\cos _{\lambda}(t)$ e seno, $\operatorname{sen}_{\lambda}(t)$, de tal forma que

$$
\begin{aligned}
\cos _{\lambda}(\omega t) & =\frac{\mathrm{e}_{\lambda}(i \omega t)+\mathrm{e}_{\lambda}(-i \omega t)}{2} ; \\
\operatorname{sen}_{\lambda}(\omega t) & =\frac{\mathrm{e}_{\lambda}(i \omega t)-\mathrm{e}_{\lambda}(-i \omega t)}{2 i} .
\end{aligned}
$$

Nas duas definições, tem-se que, quando $\lambda \rightarrow 0$, as funções tradicionais $\cos (\omega t)$ e $\operatorname{sen}(\omega t)$ são reobtidas. A Fig. 5.2 apresenta o comportamento de algumas curvas para estas famílias de funções. Ao observar tais gráficos abre-se espaço para a reflexão sobre generalizações consistentes. É importante destacar que, nestes casos, nem todas as curvas destas famílias de funções preservam algumas das principais características de uma função trigonométrica tradicional, já que apenas para $\lambda=0$ obtém-se relações limitadas e que oscilam. Por outro lado estas funções são contínuas e reais para qualquer valor do parâmetro $\lambda$. Além disso, elas preservam a paridade (ou imparidade), já que:

$$
\begin{aligned}
\cos _{\lambda}(-\omega t) & =\cos _{\lambda}(\omega t) \\
\operatorname{sen}_{\lambda}(-\omega t) & =-\operatorname{sen}_{\lambda}(\omega t) .
\end{aligned}
$$

Outras propriedades válidas para a versão usual são estendidas para a generalizada [32], como, por exemplo, o princípio fundamental da trigonometria que, neste caso, fica definido por

$$
\cos _{\lambda}^{2}(\omega t)+\operatorname{sen}_{\lambda}^{2}(\omega t)=\mathrm{e}_{\lambda}\left(\lambda \omega^{2} t^{2}\right)
$$

Quando $\lambda=0$, retorna ao resultado original. Desta forma, tem-se um "princípio fundamental" diferente para cada valor de $\lambda$, o que pode causar certa estranheza. Em contrapartida, essa generalização mantém válido o limite fundamental do seno, já que

$$
\lim _{t \rightarrow 0} \frac{\operatorname{sen}_{\lambda}(\omega t)}{t}=\omega, \quad \forall \lambda, \omega, t \in \mathbb{R} .
$$



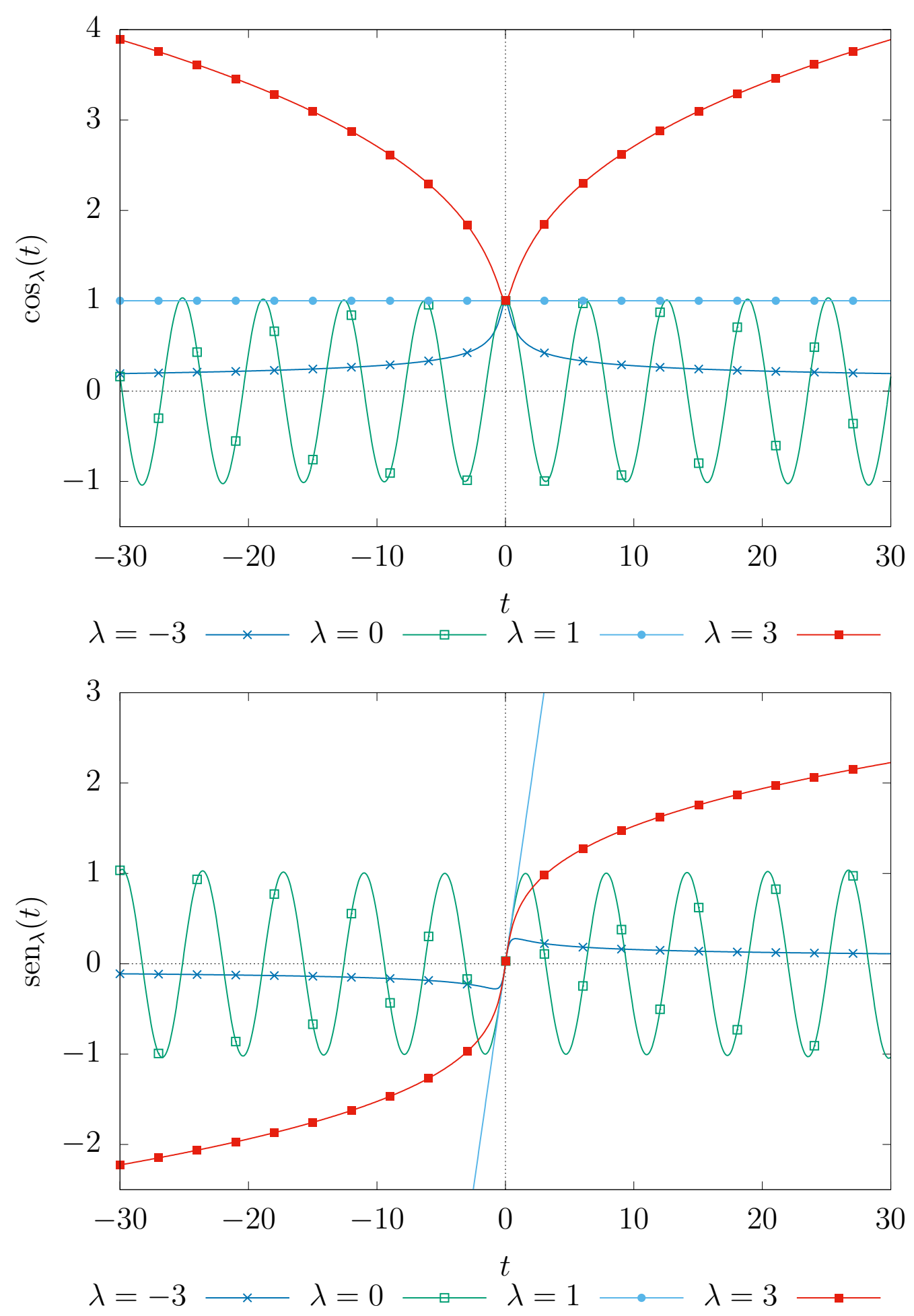

Figura 5.2: (a) Função cosseno generalizado com valores de $\lambda=0, \lambda=1, \lambda=3$ e $\lambda=-3$. (b) Função seno generalizado com valores de $\lambda=0, \lambda=1, \lambda=3$ e $\lambda=-3$. Em ambos exemplos foram obtidas funções oscilatórias e limitadas, apenas para $\lambda=0$. As demais curvas não são limitadas e não oscilam. 
Uma relação interessante, válida também na versão generalizada, é a relação de Euler. De modo muito semelhante à versão original, tem-se que:

$$
\mathrm{e}_{\lambda}( \pm i \omega t)=\cos _{\lambda}(\omega t) \pm i \operatorname{sen}_{\lambda}(\omega t)
$$

Utilizando este resultado, chega-se a uma versão generalizada para a fórmula de De Moivre, dada por

$$
\begin{aligned}
{\left[\mathrm{e}_{\lambda}( \pm i \omega t)\right]^{n} } & =\left[\cos _{\lambda}(\omega t) \pm i \operatorname{sen}_{\lambda}(\omega t)\right]^{n}= \\
& =\cos _{\lambda / n}(n \omega t) \pm i \operatorname{sen}_{\lambda / n}(n \omega t)= \\
& =\mathrm{e}_{\lambda / n}( \pm i n \omega t) .
\end{aligned}
$$

No que se refere ao cálculo diferencial, as funções $\cos _{\lambda}(\omega t)$ e $\operatorname{sen}_{\lambda}(\omega t)$ apresentam, segundo Gonzalez [32], expressões um tanto quanto rebuscadas, à saber:

$$
\begin{aligned}
\frac{d^{n} \cos _{\lambda}(t)}{d t^{n}} & =\frac{\left[\mathrm{e}_{\lambda}(-i t)\right]^{1-n \lambda}+(-1)^{n}\left[\mathrm{e}_{\lambda}(i t)\right]^{1-n \lambda}}{2} \frac{(i \lambda)^{n} \Gamma(n-1 / \lambda)}{\Gamma(-1 / \lambda)} \\
& =\frac{(-\lambda)^{n} \Gamma(n-1 / \lambda)}{\Gamma(-1 / \lambda)}\left\{\begin{array}{l}
\operatorname{sen}_{\lambda /(1-n \lambda)}[(1-n \lambda) t], n=1,3,5, \cdots, \\
\cos _{\lambda /(1-n \lambda)}[(1-n \lambda) t], n=2,4,6, \cdots
\end{array},\right. \\
\frac{d^{n} \operatorname{sen}_{\lambda}(t)}{d t^{n}} & =\frac{\left[\mathrm{e}_{\lambda}(-i t)\right]^{1-n \lambda}+(-1)^{n}\left[\mathrm{e}_{\lambda}(i t)\right]^{1-n \lambda}}{2} \frac{i(i \lambda)^{n} \Gamma(n-1 / \lambda)}{\Gamma(-1 / \lambda)} \\
& =\frac{(-\lambda)^{n} \Gamma(n-1 / \lambda)}{\Gamma(-1 / \lambda)}\left\{\begin{array}{l}
\cos _{\lambda /(1-n \lambda)}[(1-n \lambda) t], n=1,3,5, \cdots \\
\operatorname{sen}_{\lambda /(1-n \lambda)}[(1-n \lambda) t], n=2,4,6, \cdots
\end{array},\right.
\end{aligned}
$$

$\operatorname{com} \Gamma(t)$, a função gama. Tais expressões, quando $\lambda \rightarrow 0$ retomam as derivadas das funções tradicionais $\cos _{0}(t)=\cos (t)$ e $\operatorname{sen}_{0}(t)=\operatorname{sen}(t)$.

Sobre as integrais de $\cos _{\lambda}(t)$ e $\operatorname{sen}_{\lambda}(t)$ o resultado obtido é

$$
\begin{aligned}
\int \cos _{\lambda}(t) d t & =\frac{(\lambda t-i)(1+i \lambda t)^{\frac{1}{\lambda}}+(\lambda t+i)(1-i \lambda t)^{\frac{1}{\lambda}}}{2(\lambda+1)}+C_{1}= \\
& =\frac{(1-i \lambda t) \mathrm{e}_{\lambda}(i t)-(1+i \lambda t) \mathrm{e}_{\lambda}(-i t)}{2 i(1+\lambda)}+C_{1}, \\
\int \operatorname{sen}_{\lambda}(t) d t & =-\frac{(1+i \lambda t)^{\frac{1}{\lambda}+1}+(1-i \lambda t)^{\frac{1}{\lambda}+1}}{2(\lambda+1)}+C_{2}= \\
& =-\frac{(1+i \lambda t) \mathrm{e}_{\lambda}(i t)+(1-i \lambda t) \mathrm{e}_{\lambda}(-i t)}{2(1+\lambda)}+C_{2} .
\end{aligned}
$$

A Eq.( 5.35) lembra a expressão que define o seno, já que possui a diferença entre uma exponencial positiva e outra negativa, porém não é possível obter uma 
expressão concisa em termos de $\operatorname{sen}_{\lambda}(t)$. De forma equivalente, a Eq. (5.36) remete a algo de cosseno, porém também não é possível uma simplificação. As duas expressões reobtém a integral das funções seno e cosseno quando $\lambda \rightarrow 0$.

Com as propriedades apresentadas, pode-se verificar que existem prós e contras a essa generalização das funções trigonométricas. Aponta-se como maior crítica o fato de que tais funções não são limitadas nem oscilantes para qualquer valor do parâmetro $\lambda$.

As funções hiperbólicas são generalizadas de forma semelhante às trigonométricas circulares, à saber:

$$
\begin{aligned}
\cosh _{\lambda}(\omega t) & =\frac{\mathrm{e}_{\lambda}(\omega t)+\mathrm{e}_{\lambda}(-\omega t)}{2}, \\
\operatorname{senh}_{\lambda}(\omega t) & =\frac{\mathrm{e}_{\lambda}(\omega t)-\mathrm{e}_{\lambda}(-\omega t)}{2} .
\end{aligned}
$$

Por esse motivo, acabam tendo suas características e propriedades compatíveis com as que foram obtidas nas funções $\cos _{\lambda}(t)$ e $\operatorname{sen}_{\lambda}(t)$.

\subsubsection{Novas generalizações}

As funções trigonométricas generalizadas apresentadas na Sec. 5.2.1 partem da relação:

$$
\mathrm{e}^{i \omega t} \rightarrow \mathrm{e}_{\lambda}(i \omega t)
$$

Contudo, essa não é a única correspondência possível. Formas como $\left[\mathrm{e}_{\lambda}(\omega t)\right]^{i}$ ou $\left[\mathrm{e}_{\lambda}(t)\right]^{i \omega}$ também se prestam a esse papel. Aliás, ao analisar-se o núcleo da $\lambda$-TF, apresentada tanto no Cap. 4, quanto na Ref. [23], como

$$
\mathcal{F}_{\lambda}[f]=\frac{1}{\sqrt{4 \pi}} \int_{-\infty}^{\infty} \frac{\left[\mathrm{e}_{\lambda}(t)\right]^{i \omega}}{|1+\lambda t|} f(t) d t
$$

é possível obter uma interessante relação. A expressão, $\left[\mathrm{e}_{\lambda}(t)\right]^{i \omega}$, em que $\mathrm{e}_{\lambda}(t)=$ $(1+\lambda t)^{1 / \lambda}$ é a função exponencial generalizada, abre espaço para uma nova reflexão sobre as generalizações das funções trigonométricas e as relaciona com funções logperiódicas, já que

$$
\left[\mathrm{e}_{\lambda}(t)\right]^{i \omega}=(1+\lambda t)^{\frac{i \omega}{\lambda}}=\mathrm{e}^{\frac{i \omega}{\lambda} \ln (1+\lambda t)}
$$

que, com o uso da relação de Euler, pode ser escrita como

$$
\left[\mathrm{e}_{\lambda}(t)\right]^{i \omega}=\cos \left[\frac{\omega}{\lambda} \ln (1+\lambda t)\right]+i \operatorname{sen}\left[\frac{\omega}{\lambda} \ln (1+\lambda t)\right],
$$


ou ainda,

$$
\left[\mathrm{e}_{\lambda}(t)\right]^{i \omega}=\cos \left\{\omega \ln \left[\mathrm{e}_{\lambda}(t)\right]\right\}+i \operatorname{sen}\left\{\omega \ln \left[\mathrm{e}_{\lambda}(t)\right]\right\} .
$$

Desta forma, a Eq. (5.41) revela que o núcleo da $\lambda$-TF é na verdade composto por duas funções log-periódicas: uma no seno e a outra no cosseno. Este fato abre espaço para diversas discussões acerca do uso de conceitos e propriedades nos dois campos. Uma dessas possibilidades é a extensão das séries de Fourier para uma versão generalizada. Um outro resultado, também muito importante, pode ser visto na Eq. (5.42), que generaliza as funções log-periódicas em ligação direta à função exponencial generalizada $\mathrm{e}_{\lambda}(t)$.

Estudando de modo mais profundo a primeira parcela do lado direito da Eq. (5.41) pode-se perceber que:

$$
\begin{aligned}
\cos \left[\frac{\omega}{\lambda} \ln (1+\lambda t)\right] & =\frac{\mathrm{e}^{\frac{i \omega}{\lambda} \ln (1+\lambda t)}+\mathrm{e}^{\frac{-i \omega}{\lambda} \ln (1+\lambda t)}}{2}=\frac{(1+\lambda t)^{\frac{i \omega}{\lambda}}+(1+\lambda t)^{\frac{-i \omega}{\lambda}}}{2}= \\
& =\frac{\left[\mathrm{e}_{\lambda}(t)\right]^{i \omega}+\left[\mathrm{e}_{\lambda}(t)\right]^{-i \omega}}{2} .
\end{aligned}
$$

Já a expressão que envolve a função seno na segunda parcela resulta em:

$$
\begin{aligned}
\operatorname{sen}\left[\frac{\omega}{\lambda} \ln (1+\lambda t)\right] & =\frac{\mathrm{e}^{\frac{i \omega}{\lambda} \ln (1+\lambda t)}-\mathrm{e}^{\frac{-i \omega}{\lambda} \ln (1+\lambda t)}}{2 i}=\frac{(1+\lambda t)^{\frac{i \omega}{\lambda}}-(1+\lambda t)^{\frac{-i \omega}{\lambda}}}{2 i}= \\
& =\frac{\left[\mathrm{e}_{\lambda}(t)\right]^{i \omega}-\left[\mathrm{e}_{\lambda}(t)\right]^{-i \omega}}{2 i} .
\end{aligned}
$$

As equações Eq. (5.43) e Eq. (5.44), escritas em termos da função exponencial generalizada, são possibilidades generalizações das funções trigonométricas, cosseno e seno pela relação $\mathrm{e}^{i \omega t}=\left[\mathrm{e}^{t}\right]^{i \omega} \rightarrow\left[\mathrm{e}_{\lambda}(t)\right]^{i \omega}$ ao invés de $\mathrm{e}^{i \omega t} \rightarrow \mathrm{e}_{\lambda}(i \omega t)$ utilizada na generalização anterior. Desta forma, e por facilidade de notação, as novas generalizações das funções cosseno $c_{\lambda}(\omega, t)$ e seno $s_{\lambda}(\omega, t)$, propostas por nós, como funções de duas variáveis, serão doravante indicadas respectivamente por:

$$
\begin{aligned}
& \mathrm{c}_{\lambda}(\omega, t)=\frac{\left[\mathrm{e}_{\lambda}(t)\right]^{i \omega}+\left[\mathrm{e}_{\lambda}(t)\right]^{-i \omega}}{2}=\cos \left\{\omega \ln \left[\mathrm{e}_{\lambda}(t)\right]\right\} \\
& \mathrm{s}_{\lambda}(\omega, t)=\frac{\left[\mathrm{e}_{\lambda}(t)\right]^{i \omega}-\left[\mathrm{e}_{\lambda}(t)\right]^{-i \omega}}{2 i}=\operatorname{sen}\left\{\omega \ln \left[\mathrm{e}_{\lambda}(t)\right]\right\} .
\end{aligned}
$$

A variável $\omega$ será chamada de frequência angular enquanto $t$ é a variável principal. Não é conveniente pensar em $\omega$ como parâmetro, pois como variável satisfaz propriedades importantes, como a paridade, soma de arcos, entre outras, mostradas mais adiante. 
Destacamos aqui os estudos desenvolvidos por Scarfone [67, 82] e por Kim [83] que validam nossa abordagem, partindo de motivações distintas. No entanto, esses estudos não apresentam uma discussão mais aprofundada das propriedades dessas funções. Deve-se mencionar, que estes estudos apresentam as generalizações com um único argumento, o que pode levar a inconsistências nas demonstrações de propriedades.

De volta às nossas generalizações $c_{\lambda}(\omega, t)$ e $\mathrm{s}_{\lambda}(\omega, t)$, pode-se observar o comportamento de algumas curvas pertencentes à essas funções nos gráficos apresentados na Fig. 5.3. Nesta figura é possível verificar que, tanto $c_{\lambda}(\omega, t)$ quanto $\mathrm{s}_{\lambda}(\omega, t)$, oscilam no intervalo $[-1,1]$. Essa é uma característica peculiar das funções cosseno e seno tradicionais. Contudo, existe um valor crítico: para $\lambda t<-1$ tanto $c_{\lambda}(\omega, t)$ quanto $\mathrm{s}_{\lambda}(\omega, t)$ não são reais. Este fato poderia indicar certa fragilidade de nossa definição, já que as funções trigonométricas tradicionais são reais em todo seu domínio. No entanto, o que de fato aparecem aqui são pontos de acumulação, tão importantes nas funções log-periódicas, conforme já destacado, que ganham mais expressividade ainda, já que variam para cada valor de $\lambda$. Desta forma, essa proposta estende também o conceito de funções log-periódicas com "pontos de acumulação generalizados", indicando que essas novas generalizações das funções trigonométricas são também funções log-periódicas generalizadas sob o parâmetro $\lambda$. Nos exemplos apresentados na Fig. 5.3 é possível observar pontos de acumulação entre -1 e 0 . De modo mais preciso, estes pontos estão em $t=-1, t=-1 / 2$ e $t=-1 / 3$ para os valores de $\lambda=1, \lambda=2$ e $\lambda=3$ respectivamente. Características semelhante seriam obtidas se a plotagem considerasse, simultaneamente, $\lambda<0$ e $t<0$.

Outra característica muito importante é observada quando analisa-se as partes reais e imaginárias de $c_{\lambda}(\omega, t)$ e $\mathrm{s}_{\lambda}(\omega, t)$ na região onde $\lambda t<-1$. É possível verificar que, também neste caso, ambas oscilam, são limitadas e possuem ponto de acumulação, como pode ser observado nas Figs. 5.4 e 5.5. A Fig. 5.4 mostra ainda que, ao plotar, por exemplo, as partes imaginárias das funções $c_{\lambda}(\omega, t)$ e $\mathrm{s}_{\lambda}(\omega, t)$ vê-se que o intervalo de oscilação é o mesmo para as duas funções.

É possível observar, ainda, que os limites inferior e superior diferem de um valor de $\lambda$ para outro. A Fig. 5.5 apresenta um exemplo da parte imaginária da função $\mathrm{c}_{\lambda}(5, t)$ para valores de $\lambda=2.0, \lambda=2.25$ e $\lambda=2.5$. 

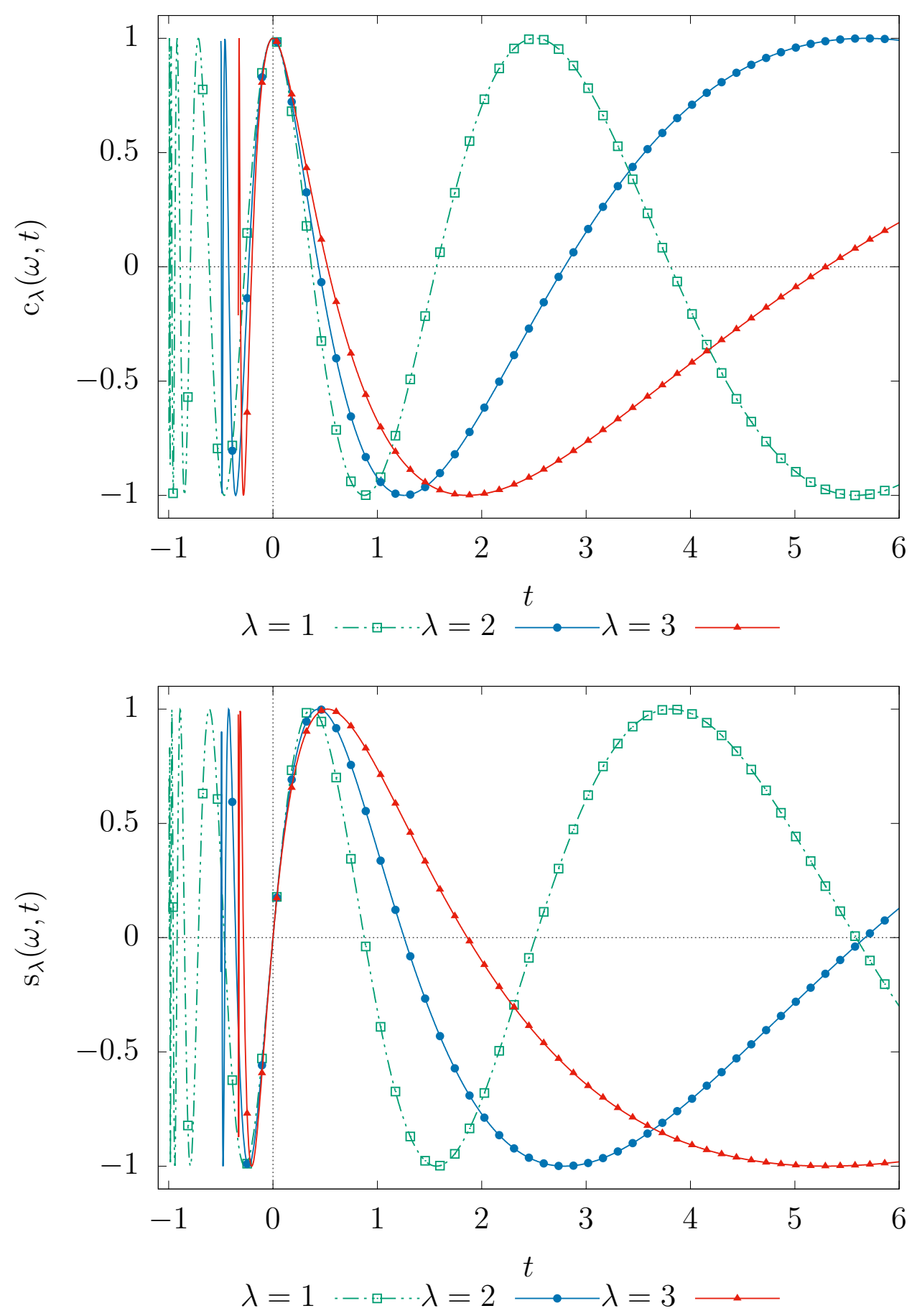

Figura 5.3: (a) Nova generalização para a função cosseno com valores de $\lambda=1, \lambda=2, \lambda=3$ e $\omega=5$. (b) Nova generalização para a função seno com valores de $\lambda=1, \lambda=2, \lambda=3$ e $\omega=5$. Em ambos exemplos foram obtidas funções oscilatórias e limitadas, característica peculiar das funções trigonométricas. Além disso, é observável, nas duas funções, pontos de acumulação em $t=-1 / \lambda$. 


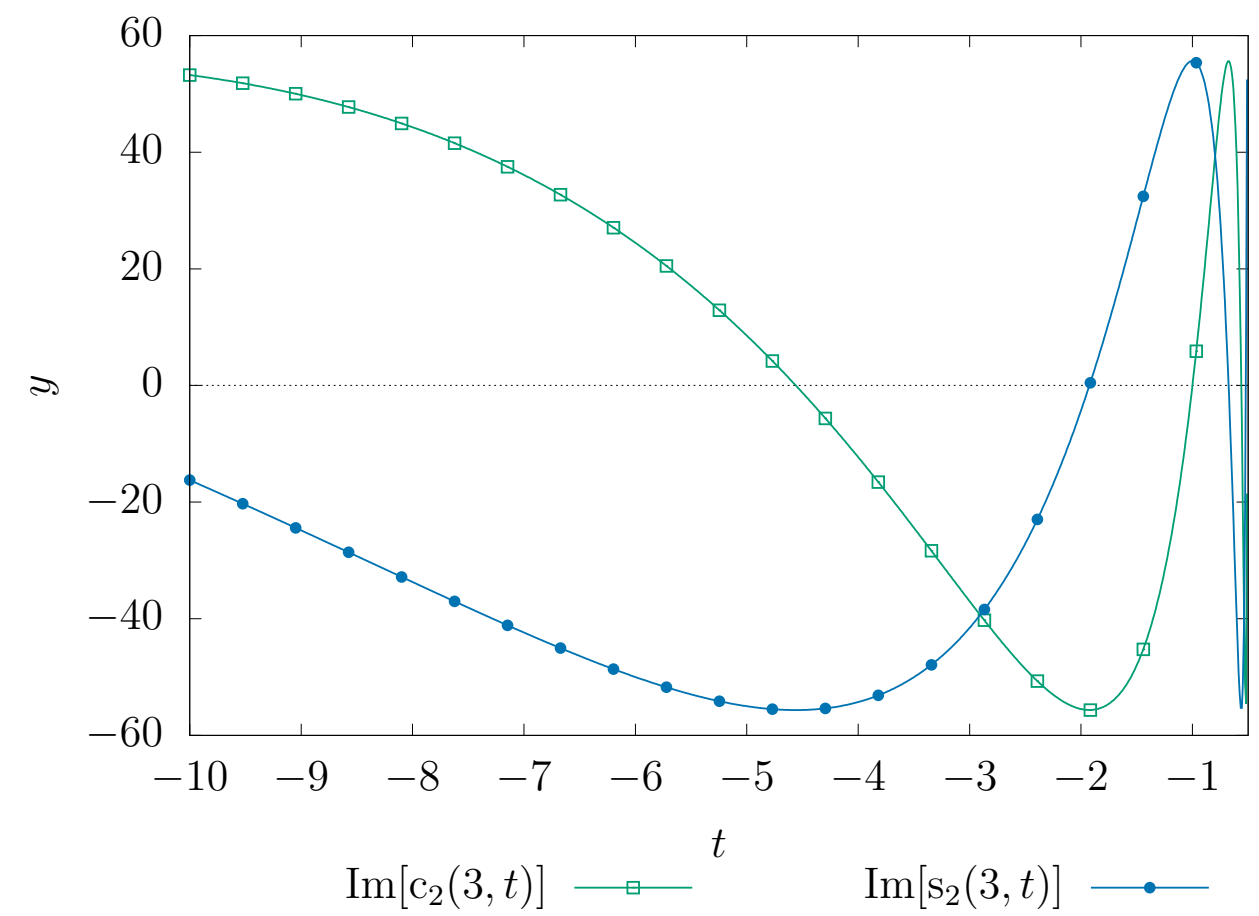

Figura 5.4: Partes imaginárias das funções $\mathrm{c}_{2}(3, t)$ e $\mathrm{s}_{2}(3, t)$ - o conjunto imagem das duas funções é o mesmo. Observa-se ainda ponto de acumulação próximo da origem (mais precisamente em $t=-1 / 3)$.

Outra importante afirmação a respeito das curvas apresentadas na Fig. 5.5 é relativa à imagem da função. Seja $I$ este conjunto. A primeira observação à ser feita é que para valores de $\lambda$ adequados, $I$ é sempre simétrico em relação ao eixo das abcissas, conforme o que ocorre nas funções tradicionais. Desta forma,

$$
I:\{y \in \mathbb{R} \backslash-r \leq y \leq r\}
$$

para um valor real $r$ determinado. Uma segunda observação é que, quanto maior o valor de $\lambda$, menor o valor $r$ no conjunto imagem da função. Por este fato, o parâmetro teve pouca variação nos exemplos apresentados na Fig. 5.5. Fixando-se $\omega$, à medida que $\lambda \rightarrow \infty$ observa-se que as partes real e imaginária das duas funções tendem à constantes, porém em duas situações distintas: na função $\mathrm{s}_{\lambda}(\omega, t)$ verificase que tanto $\operatorname{Re}\left(\mathrm{s}_{\lambda}(\omega, t)\right)$ quanto $\operatorname{Im}\left(\mathrm{s}_{\lambda}(\omega, t)\right)$ vão pra zero, de modo que a parte imaginária é um pouco mais rápida que a parte real; já na função $c_{\lambda}(\omega, t)$ verifica-se que $\operatorname{Re}\left(c_{\lambda}(\omega, t)\right)$ tende a 1 quando $\lambda \rightarrow \infty$ e $\operatorname{Im}\left(c_{\lambda}(\omega, t)\right)$ vai a 0 . Essa afirmação é exemplificada na Fig. 5.6.

A frequência angular $\omega$ tem relação direta com o conjunto imagem $I$ da 


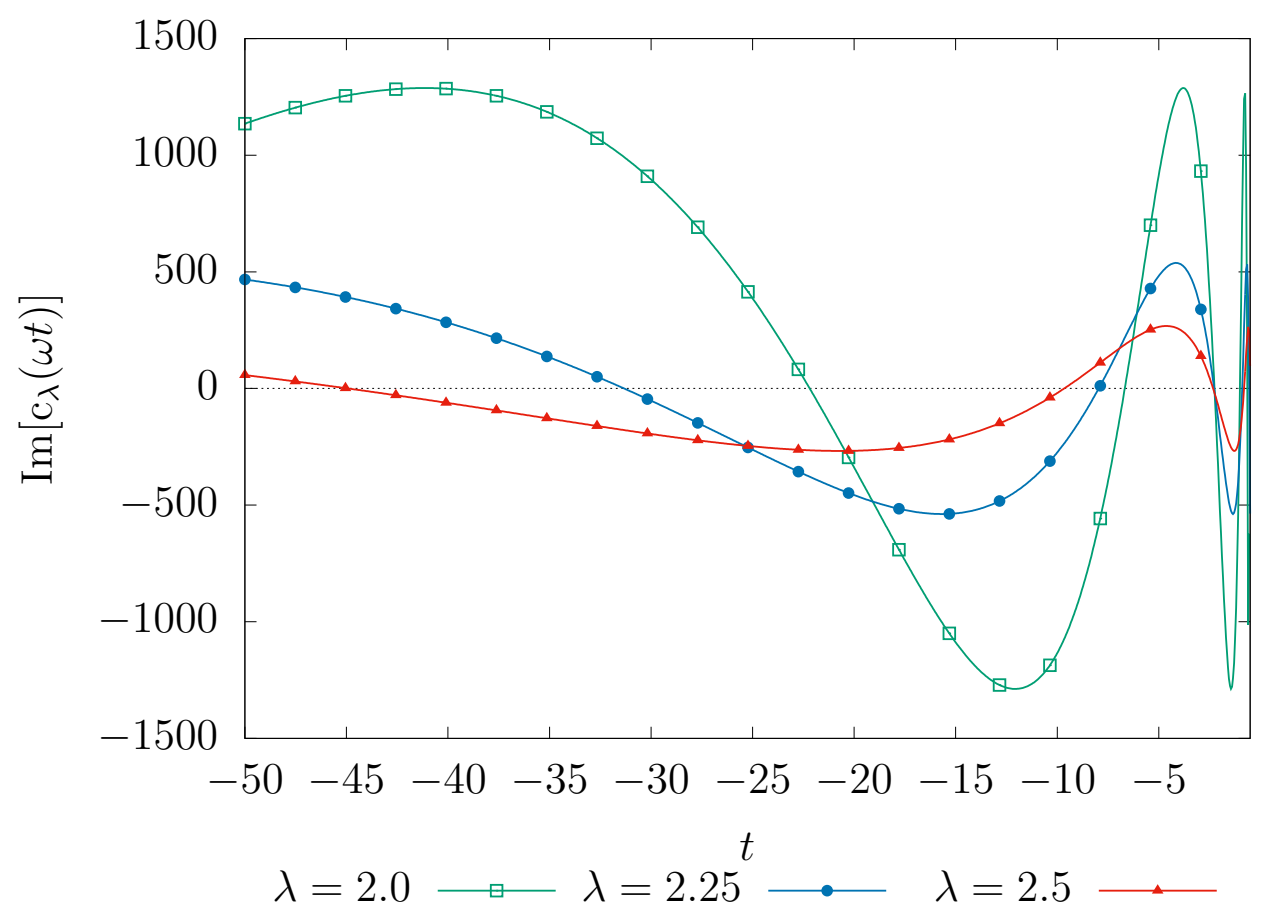

Figura 5.5: Parte imaginária da função $c_{\lambda}(5, t)$ para $\lambda=2.0, \lambda=2.25$ e $\lambda=2.5$. Função se mantém limitada e oscilatória para cada valor $\lambda$. Observa-se ponto de acumulação próximo à origem.

Eq. (5.46): quanto maior $\omega$, maior será $r$. Esta última observação pode ser verificada na Fig. 5.7, onde plota-se a parte real da função $s_{3}(\omega, t)$ para $\omega=3, \omega=4$ e $\omega=5$, respectivamente.

Apresenta-se, a seguir, algumas importantes propriedades válidas para tal generalização. Neste ponto, vale destacar que as generalizações mais consistentes são aquelas que mantém as principais características e propriedades das funções originais, conforme já apresentado em seções anteriores e é o caso da generalização que propomos. As demonstrações e cálculos mais detalhados da maioria dos resultados apresentados à seguir estão disponíveis no Apêndice 3. Por facilidade de notação, quando a frequência angular $\omega=1$ escreveremos $c_{\lambda}(1, t)=c_{\lambda}(t)$ para o cosseno e $\mathrm{s}_{\lambda}(1, t)=\mathrm{s}_{\lambda}(t)$ para o seno.

\subsubsection{Paridade}

Nas funções trigonométricas tradicionais, como já indicado anteriormente, a função cosseno é uma função par e a função seno é impar. Nossa generalização 

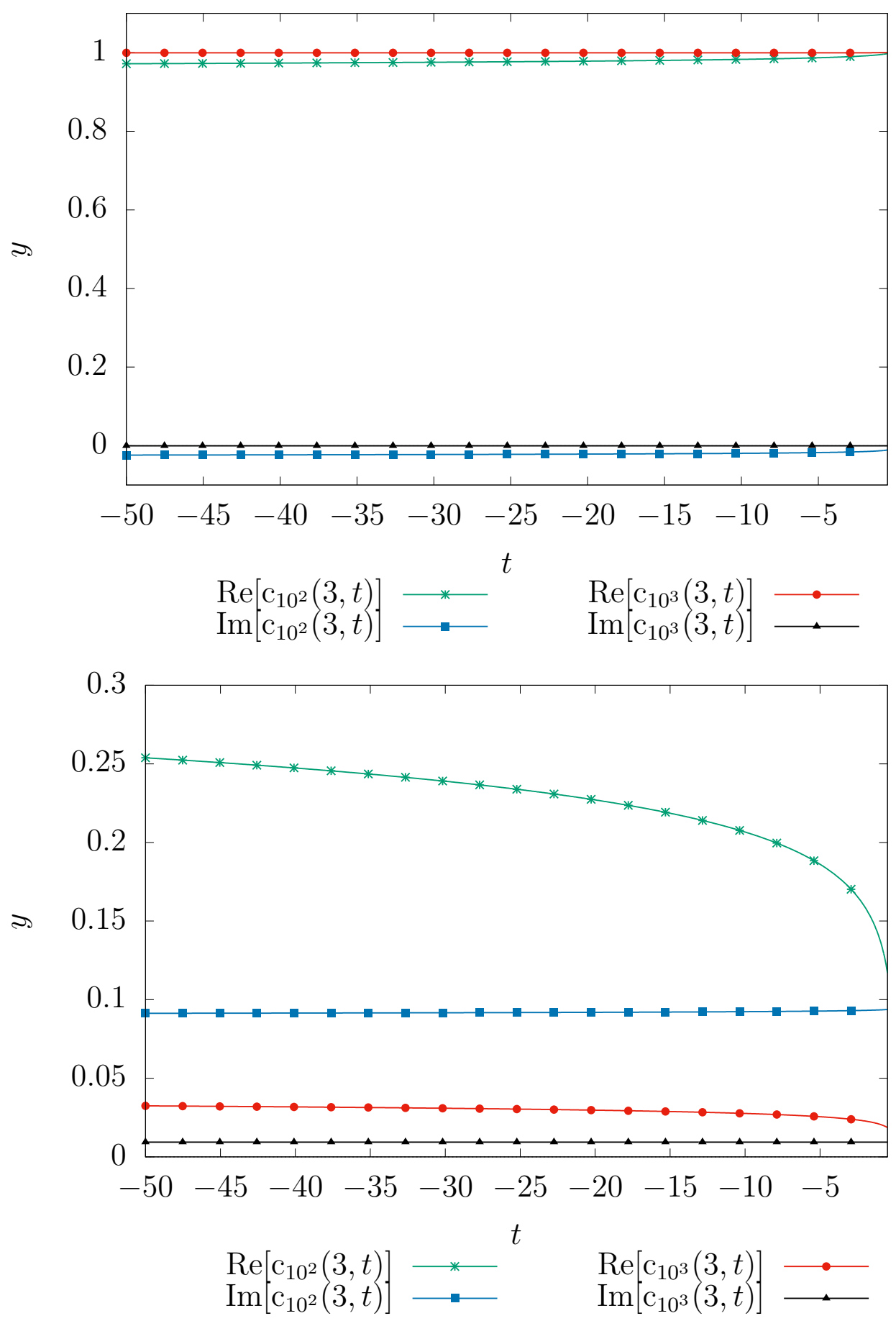

Figura 5.6: (a) Partes real e imaginária da função $c_{\lambda}(3, t)$ para $\lambda \rightarrow \infty$. A Parte real claramente tende a 1 enquanto a imaginária a 0 . (b) Partes real e imaginária da função $\mathrm{s}_{\lambda}(3, t)$ para $\lambda \rightarrow \infty$. Tanto parte real quanto imaginária tendem a 0.

mantém a paridade na frequência angular $\omega$, já que

$$
c_{\lambda}(-\omega, t)=\frac{\left[\mathrm{e}_{\lambda}(t)\right]^{-i \omega}+\left[\mathrm{e}_{\lambda}(t)\right]^{i \omega}}{2}=\mathrm{c}_{\lambda}(\omega, t),
$$




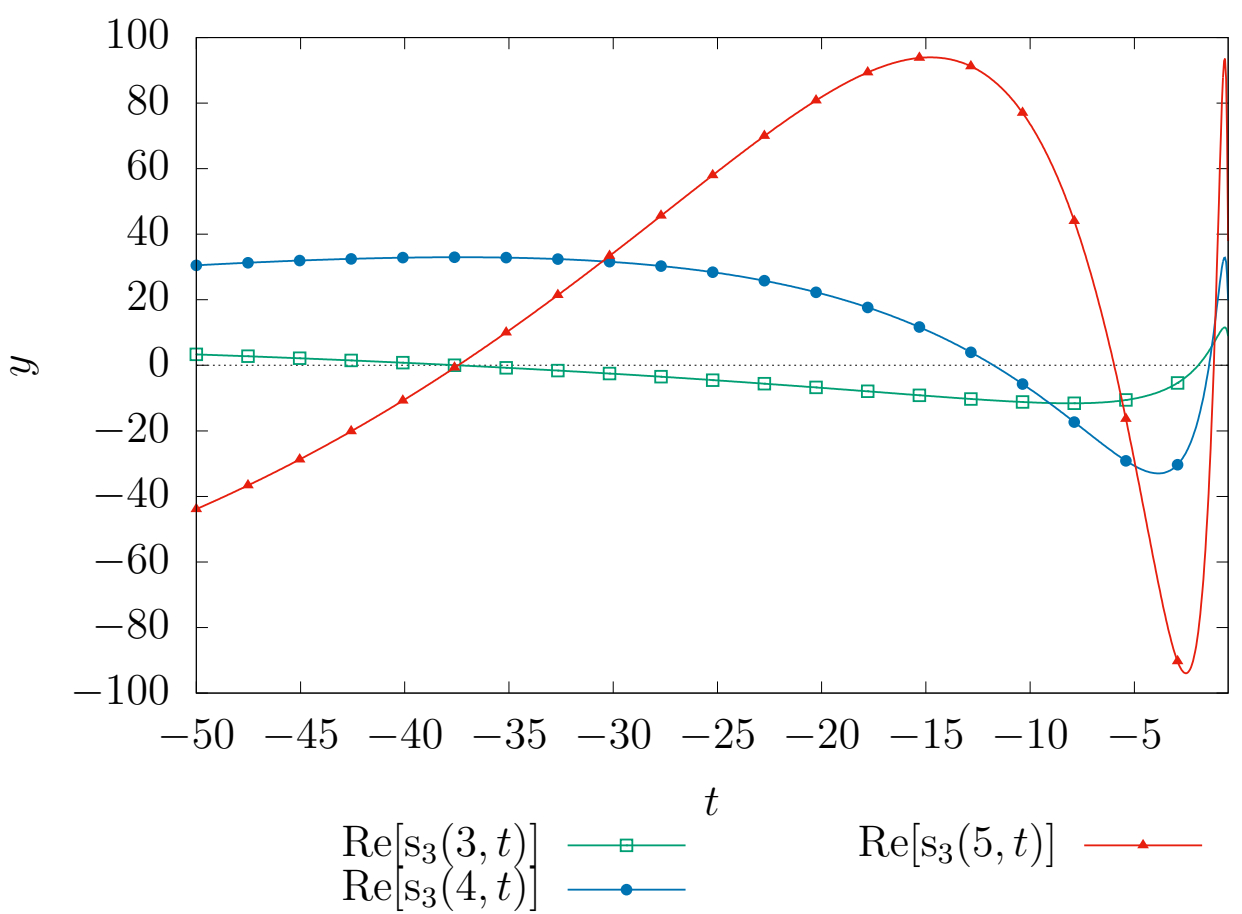

Figura 5.7: Parte real da função $\mathrm{s}_{3}(\omega, t)$ para $\omega=3, \omega=4$ e $\omega=5$. Função se mantém limitada e oscilatória, aumentando o intervalo do conjunto imagem conforme o aumento do valor da frequência $\omega$.

como também a "imparidade" de $\mathrm{s}_{\lambda}(\omega, t)$ na mesma variável, observando que,

$$
\mathrm{s}_{\lambda}(-\omega, t)=\frac{\left[\mathrm{e}_{\lambda}(t)\right]^{-i \omega}-\left[\mathrm{e}_{\lambda}(t)\right]^{i \omega}}{2 i}=-\mathrm{s}_{\lambda}(\omega, t)
$$

Quanto à variável $t$, a paridade não é preservada para qualquer valor do parâmetro $\lambda$. Contudo, obtém-se importantes relações:

$$
\begin{aligned}
& \mathrm{c}_{\lambda}(\omega,-t)=\mathrm{c}_{-\lambda}(-\omega, t)=\mathrm{c}_{-\lambda}(\omega, t) \\
& \mathrm{s}_{\lambda}(\omega,-t)=\mathrm{s}_{-\lambda}(-\omega, t)=-\mathrm{s}_{-\lambda}(\omega, t),
\end{aligned}
$$

que mostram uma relação entre a variável $t$ e o parâmetro $\lambda$ nessas funções. Quando $\lambda \rightarrow 0$ reobtemos a paridade das funções trigonométricas.

\subsubsection{Relação Fundamental da Trigonometria}

Uma das mais importantes propriedades da trigonometria é a relação fundamental. Ela é fruto do teorema de Pitágoras, juntamente com as definições geométricas de senos e cossenos de ângulos $0 \leq \theta \leq \pi / 2$, em que, sendo $a$ e $b$ catetos de 
um triângulo retângulo e $c$ a respectiva hipotenusa, têm-se a igualdade

$$
c^{2}=a^{2}+b^{2}
$$

Ao dividir os dois lados da equação por $c^{2}$ chega-se à relação fundamental que, para as funções tradicionais, é dada por $\operatorname{sen}^{2}(\omega t)+\cos ^{2}(\omega t)=1, \forall \omega, t \in \mathbb{R}$. É claro que tanto a propriedade quanto sua demonstração são de amplo conhecimento, mas este resultado é colocado aqui propositadamente para indicar que tal relação é proveniente de relações métricas e, desta forma, não é concebível que apresente resultado diferente do unitário.

A primeira proposta apresentou como generalização da relação fundamental a igualdade: $\cos _{\lambda}^{2}(\omega t)+\operatorname{sen}_{\lambda}^{2}(\omega t)=\mathrm{e}_{\lambda}\left(\lambda \omega^{2} t^{2}\right)$. Nesta relação, o valor unitário é preservado apenas quando $\lambda \rightarrow 0$. A nossa proposta de generalização preserva o valor unitário da relação fundamental para qualquer $\lambda$, já que

$$
c_{\lambda}^{2}(\omega, t)+s_{\lambda}^{2}(\omega, t)=1, \forall \omega, \lambda, t
$$

Esse resultado reforça a ideia de que $c_{\lambda}(\omega, t)$ e $\mathrm{s}_{\lambda}(\omega, t)$ são generalizações consistentes para $\cos (\omega t)$ e sen $(\omega t)$.

\subsubsection{Relação de Euler}

Essa relação pode ser estendida tanto para a generalização anterior, quanto para a nossa. Neste caso tem-se que

$$
\left[\mathrm{e}_{\lambda}(t)\right]^{ \pm i \omega}=\mathrm{c}_{\lambda}(\omega, t) \pm i \mathrm{~s}_{\lambda}(\omega, t)
$$

Novamente, quando $\lambda \rightarrow 0$, retomamos a relação original.

\subsubsection{Fórmula de De Moivre}

Outro resultado importante da análise complexa é a fórmula de De Moivre, que pode ser deduzida a partir da relação de Euler e por isso vale tanto na generalização anterior quanto nesta. A versão generalizada desta fórmula na nova proposta é dada por:

$$
\begin{aligned}
{\left[\mathrm{c}_{\lambda}(\omega, t) \pm i \mathrm{~s}_{\lambda}(\omega, t)\right]^{n} } & =\left\{\left[\mathrm{e}_{\lambda}(t)\right]^{ \pm i \omega}\right\}^{n}=\left\{\left[\mathrm{e}_{\lambda}(x)\right]^{n}\right\}^{ \pm i \omega}= \\
& =\left[\mathrm{e}_{\lambda / n}(n t)\right]^{ \pm i \omega}=\mathrm{c}_{\lambda / n}(\omega, n t) \pm i \mathrm{~s}_{\lambda / n}(\omega, n t) .
\end{aligned}
$$


Mais uma vez, afirma-se que generalizações mais consistentes preservam características e propriedades das funções originais. Este é mais um exemplo da eficácia da generalização proposta.

\subsubsection{Soma de $\operatorname{arcos}$}

Existem muitas situações em que algumas propriedades da trigonometria tradicional são muito úteis para simplificação de resultados, transformações de parâmetros, entre outros. A propriedade da soma de arcos é uma dessas. Na primeira generalização [31], não é possível obter propriedades semelhantes a essa. Esse fato se dá, principalmente, pelo fato do produto entre variável, frequência e unidade imaginária fazer parte do argumento de $\mathrm{e}_{\lambda}(t)$. Como essa função faz parte de uma potência, tem-se restrição de operações e simplificações possíveis.

Na nossa proposta, é possível verificar propriedades equivalentes a essa da soma de arcos de diversas maneiras. A primeira apresenta uma soma (ou diferença) generalizada de arcos e é firmada sobre a variável principal. Desta forma, verifica-se que:

$$
\begin{aligned}
& \mathrm{c}_{\lambda}\left(\omega, a \oplus_{\lambda} b\right)=\mathrm{c}_{\lambda}(\omega, a) \mathrm{c}_{\lambda}(\omega, b)-\mathrm{s}_{\lambda}(\omega, a) \mathrm{s}_{\lambda}(\omega, b), \\
& \mathrm{c}_{\lambda}\left(\omega, a \ominus_{\lambda} b\right)=\mathrm{c}_{\lambda}(\omega, a) \mathrm{c}_{\lambda}(\omega, b)+\mathrm{s}_{\lambda}(\omega, a) \mathrm{s}_{\lambda}(\omega, b), \\
& \mathrm{s}_{\lambda}\left(\omega, a \oplus_{\lambda} b\right)=\mathrm{s}_{\lambda}(\omega, a) \mathrm{c}_{\lambda}(\omega, b)+\mathrm{s}_{\lambda}(\omega, b) \mathrm{c}_{\lambda}(\omega, a), \\
& \mathrm{s}_{\lambda}\left(\omega, a \ominus_{\lambda} b\right)=\mathrm{s}_{\lambda}(\omega, a) \mathrm{c}_{\lambda}(\omega, b)-\mathrm{s}_{\lambda}(\omega, b) \mathrm{c}_{\lambda}(\omega, a),
\end{aligned}
$$

em que $\oplus_{\lambda}$ é a soma generalizada apresentada no Cap. 2, de tal forma que $a \oplus_{\lambda} b=$ $a+b+\lambda a b$. Já $\ominus_{\lambda}$ é a diferença generalizada apresentada no mesmo capítulo, em que $a \ominus_{\lambda} b=(a-b) /(1+\lambda b)$.

Também é possível se obter uma relação de soma na variável da frequência angular $\omega$. Neste caso as relações podem ser escritas como:

$$
\begin{aligned}
& \mathrm{c}_{\lambda}\left(\omega_{1} \pm \omega_{2}, t\right)=\mathrm{c}_{\lambda}\left(\omega_{1}, t\right) \mathrm{c}_{\lambda}\left(\omega_{2}, t\right) \mp \mathrm{s}_{\lambda}\left(\omega_{1}, t\right) \mathrm{s}_{\lambda}\left(\omega_{2}, t\right), \\
& \mathrm{s}_{\lambda}\left(\omega_{1} \pm \omega_{2}, t\right)=\mathrm{s}_{\lambda}\left(\omega_{1}, t\right) \mathrm{c}_{\lambda}\left(\omega_{2}, t\right) \pm \mathrm{s}_{\lambda}\left(\omega_{2}, t\right) \mathrm{c}_{\lambda}\left(\omega_{1}, t\right),
\end{aligned}
$$

$\mathrm{Na}$ trigonometria usual, obtém-se pela soma de arcos uma propriedade conhecida como arco duplo. Nela, verifica-se que $\cos (2 a)=\cos ^{2}(a)-\operatorname{sen}^{2}(a)$ e $\operatorname{sen}(2 a)=2 \operatorname{sen}(a) \cos (a)$. Na versão generalizada, observa-se um resultado equi- 
valente, dado por

$$
\begin{aligned}
& \mathrm{c}_{\lambda}(2 \omega, a)=\mathrm{c}_{\lambda}^{2}(\omega, a)-\mathrm{s}_{\lambda}^{2}(\omega, a)=\mathrm{c}_{\lambda}\left(\omega, a \oplus_{\lambda} a\right) ; \\
& \mathrm{s}_{\lambda}(2 \omega, a)=2 \mathrm{c}_{\lambda}(\omega, a) \mathrm{s}_{\lambda}(\omega, a)=\mathrm{s}_{\lambda}\left(\omega, a \oplus_{\lambda} a\right) .
\end{aligned}
$$

Em todas as propriedades apresentadas nas expressões acima, quando $\lambda \rightarrow 0$, retorna-se às propriedades das funções trigonométricas.

Outra propriedade interessante é a que estabelece uma relação entre os produtos das variáveis por $n \in \mathbb{R}$ : $n t$ e $n \omega$. Utilizando o fato de que $\left[\mathrm{e}_{\lambda}(t)\right]^{n}=\mathrm{e}_{\lambda / n}(n t)$, verificam-se as seguintes igualdades:

$$
\begin{aligned}
& \mathrm{c}_{\lambda}(\omega, n t)=\mathrm{c}_{n \lambda}(n \omega, t) \\
& \mathrm{s}_{\lambda}(\omega, n t)=\mathrm{s}_{n \lambda}(n \omega, t) .
\end{aligned}
$$

Desta forma, verifica-se a extensão de importantes propriedades das funções trigonométricas para as generalizadas indicando que de fato temos uma generalização consistente. Na proposta de generalização de Borges [31], não foi possível encontrar propriedade equivalente para estas. Na sequência, são apresentadas mais algumas características válidas para esta proposta de generalização.

\subsubsection{Produtos}

Muitas vezes, na resolução de problemas matemáticos, obtém-se produtos de funções trigonométricas. Em várias situações, este resultado pode ser inconveniente, como por exemplo, no cálculo de uma integral. As propriedades apresentadas nas Eqs. (5.9) podem ser muito úteis para a trigonometria usual. Na nossa proposta de generalização, existem propriedades compatíveis com estas, mas com os operadores soma e subtração generalizados. Estas propriedades são dadas por:

$$
\begin{aligned}
& \mathrm{s}_{\lambda}(\omega, a) \mathrm{c}_{\lambda}(\omega, b)=\frac{1}{2}\left[\mathrm{~s}_{\lambda}\left(\omega, a \oplus_{\lambda} b\right)+\mathrm{s}_{\lambda}\left(\omega, a \ominus_{\lambda} b\right)\right] \\
& \mathrm{s}_{\lambda}(\omega, a) \mathrm{s}_{\lambda}(\omega, b)=-\frac{1}{2}\left[\mathrm{c}_{\lambda}\left(\omega, a \oplus_{\lambda} b\right)-\mathrm{c}_{\lambda}\left(\omega, a \ominus_{\lambda} b\right)\right] \\
& \mathrm{c}_{\lambda}(\omega, a) \mathrm{c}_{\lambda}(\omega, b)=\frac{1}{2}\left[\mathrm{c}_{\lambda}\left(\omega, a \oplus_{\lambda} b\right)+\mathrm{c}_{\lambda}\left(\omega, a \ominus_{\lambda} b\right)\right] .
\end{aligned}
$$

Outra propriedade, relacionada ao produto, que pode ser bastante útil nessa generalização foca na variável de frequência angular $\omega$. Neste caso, em que os 
operadores generalizados não aparecem, tem-se que

$$
\begin{aligned}
& \mathrm{s}_{\lambda}\left(\omega_{1}, t\right) \mathrm{c}_{\lambda}\left(\omega_{2}, t\right)=\frac{1}{2}\left[\mathrm{~s}_{\lambda}\left(\omega_{1}+\omega_{2}, t\right)+\mathrm{s}_{\lambda}\left(\omega_{1}-\omega_{2}, t\right)\right] \\
& \mathrm{s}_{\lambda}\left(\omega_{1}, t\right) \mathrm{s}_{\lambda}\left(\omega_{2}, t\right)=\frac{1}{2}\left[-\mathrm{c}_{\lambda}\left(\omega_{1}+\omega_{2}, t\right)+\mathrm{c}_{\lambda}\left(\omega_{1}-\omega_{2}, t\right)\right] \\
& \mathrm{c}_{\lambda}\left(\omega_{1}, t\right) \mathrm{c}_{\lambda}\left(\omega_{2}, t\right)=\frac{1}{2}\left[\mathrm{c}_{\lambda}\left(\omega_{1}+\omega_{2}, t\right)+\mathrm{c}_{\lambda}\left(\omega_{1}-\omega_{2}, t\right)\right] .
\end{aligned}
$$

Aqui, é importante destacar que o fato desta proposta utilizar $\left[\mathrm{e}_{\lambda}(t)\right]^{i \omega}$ como generalização de $\mathrm{e}^{i \omega t}$ abre espaço para análise de novas propriedades na versão generalizada que seriam compatíveis com a original. Isso só foi possível perante a nova proposta de generalização de $e_{\lambda}(t)$, apresentada no Cap. 2. Na sequência verifica-se o comportamento de $c_{\lambda}(\omega, t)$ e $s_{\lambda}(\omega, t)$ no que se refere ao cálculo diferencial e integral.

\subsubsection{Derivadas}

Desde que, no início do Séc. XVIII, Newton e Leibniz criaram, simultaneamente e de forma independente, o cálculo diferencial e integral, o mundo das ciências exatas atingiu um novo patamar. Afinal, derivadas e integrais são utilizadas como importantes ferramentas para diversos fins. Em muitas situações, funções trigonométricas e cálculo diferencial estão intimamente ligados, tanto de maneira conceitual, como ferramenta de simplificação, ou ainda no cálculo de integrais e derivadas destas funções para mais objetivos específicos. De fato, o cálculo diferencial surgiu à partir de um problema ligado à trigonometria: encontrar a tangente de uma curva ao longo de todo seu domínio (problema da tangente). Desta maneira, é importante que, em generalizações, os resultados obtidos retomem as ideias originais.

$\mathrm{Na}$ trigonometria tradicional, as derivadas do cosseno e seno são expressões bem simples alternando as duas funções, exceto por um sinal. Na nossa generalização as derivadas da função $c_{\lambda}(\omega, t)$ são dadas por expressões complicadas:

$$
\frac{d^{n} \mathrm{c}_{\lambda}(\omega, t)}{d t^{n}}=\eta(t) \sum_{\ell=1}^{m=n}(-1)^{\ell} \frac{\omega^{2 \ell-1}}{\lambda^{2 \ell-n}}\left[\lambda \mathbb{S}(n, 2 \ell-1) \mathrm{s}_{\lambda}(\omega, t)+\omega \mathbb{S}(n, 2 \ell) \mathrm{c}_{\lambda}(\omega, t)\right]
$$

Já as da função $\mathrm{s}_{\lambda}(\omega, t)$ aparecem como:

$$
\frac{d^{n} \mathrm{~s}_{\lambda}(\omega, t)}{d t^{n}}=\eta(t) \sum_{\ell=1}^{m=n}(-1)^{\ell-1} \frac{\omega^{2 \ell-1}}{\lambda^{2 \ell-n}}\left[\lambda \mathbb{S}(n, 2 \ell-1) \mathrm{c}_{\lambda}(\omega, t)-\omega \mathbb{S}(n, 2 \ell) \mathrm{s}_{\lambda}(\omega, t)\right] .
$$

Em ambos os casos $\eta(t)=1 /(1+\lambda t)^{n}$. Já o termo $\mathbb{S}(n, m)$ se refere ao $m$-ésimo número de Stirling de primeiro tipo e ordem $n$ [84, 85]. Além disso, é possível 
perceber que as derivadas dessas funções em relação a $t$, são expansões em séries de $\mathrm{s}_{\lambda}(\omega, t)$ e $\mathrm{c}_{\lambda}(\omega, t)$.

Já em relação à variável de frequência angular, $\omega$, a expressão para a $n$-ésima derivada das funções $c_{\lambda}(\omega, t)$ e $\mathrm{s}_{\lambda}(\omega, t)$ são escritas, de modo muito mais simples, respectivamente como:

$$
\begin{aligned}
& \frac{d^{n}}{d \omega^{n}} \mathrm{c}_{\lambda}(\omega, t)=\left[\ln \left(\mathrm{e}_{\lambda}(t)\right)\right]^{n} T_{n}(\omega, t), \\
& \frac{d^{n}}{d \omega^{n}} \mathrm{~S}_{\lambda}(\omega, t)=\left[\ln \left(\mathrm{e}_{\lambda}(t)\right)\right]^{n} T_{n+1}(\omega, t),
\end{aligned}
$$

onde

$$
T_{k}(\omega, t)=\left\{\begin{array}{rcc}
\mathrm{c}_{\lambda}(\omega, t) & \text { se } & \bmod (k, 4)=1 \\
-\mathrm{s}_{\lambda}(\omega, t) & \text { se } & \bmod (k, 4)=2, \\
-\mathrm{c}_{\lambda}(\omega, t) & \text { se } & \bmod (k, 4)=3 \\
\mathrm{~s}_{\lambda}(\omega, t) & \text { se } & \bmod (k, 4)=0 .
\end{array}\right.
$$

Em todos os casos, quando $\lambda \rightarrow 0$ recupera-se os resultados próprios para as funções trigonométricas.

Ao analisar o parâmetro $\lambda$ como variável lenta, pode-se então propor a diferenciação neste termo. A primeira derivada de $c_{\lambda}(\omega, t)$ em relação à $\lambda$ é dada por expressões simples:

$$
\frac{d}{d \lambda} \mathrm{c}_{\lambda}(\omega, t)=-\frac{\omega}{\lambda}\left\{\frac{t}{1+\lambda t}-\ln \left[\mathrm{e}_{\lambda}(t)\right]\right\} \mathrm{s}_{\lambda}(\omega, t) .
$$

Já para $\mathrm{s}_{\lambda}(\omega, t)$ a derivada em relação à $\lambda$ pode ser escrita como

$$
\frac{d}{d \lambda} \mathrm{s}_{\lambda}(\omega, t)=\frac{\omega}{\lambda}\left\{\frac{t}{1+\lambda t}-\ln \left[\mathrm{e}_{\lambda}(t)\right]\right\} \mathrm{c}_{\lambda}(\omega, t)
$$

Este resultado confirma a tendência de que derivadas de funções seno (tradicional ou generalizado) sejam proporcionais ao cosseno e derivadas de cossenos resultem em termos proporcionais ao simétrico de seno. As derivadas de ordem $n$ em relação a $\lambda$, produzem expressões envolvendo as somas de todas as outras derivadas de ordem até $n-1$, muito semelhantes a encontrada na diferenciação de $e_{\lambda}(t)$ em relação a este parâmetro. Devemos, em trabalhos futuros, explorar mais estes resultados e investigar possíveis aplicações.

\subsubsection{Limite fundamental}

A nossa generalização também mantém a validade de uma outra importante propriedade da trigonometria tradicional. Essa propriedade é o limite fundamental, 
que aqui é dado por

$$
\lim _{t \rightarrow 0} \frac{\mathrm{s}_{\lambda}(\omega, t)}{t}=\omega
$$

Este limite fundamental também pode ser calculado em relação à outra variável $\omega$ e, neste caso, temos outro resultado interessante:

$$
\lim _{\omega \rightarrow 0} \frac{\mathrm{s}_{\lambda}(\omega, t)}{\omega}=\ln \left[\mathrm{e}_{\lambda}(t)\right] .
$$

Quando $\lambda \rightarrow 0$, retomamos a validade da função original, ou seja, $\lim _{\omega \rightarrow 0} \operatorname{sen}(\omega t) / \omega=t$. Tais resultados podem ser úteis em cálculos de limites de funções log-periódicas ou na $\lambda$-TF.

\subsubsection{Integração}

Desde que Bernhard Riemann resolveu o problema das áreas, a integração se tornou uma ferramenta essencial da análise. A nossa proposta de generalização apresenta integração das funções $c_{\lambda}(\omega, t)$ e $\mathrm{s}_{\lambda}(\omega, t)$, respectivamente, como

$$
\begin{aligned}
& \int d t \mathrm{c}_{\lambda}(\omega, t)=\frac{(1+\lambda t)}{\left(\lambda^{2}+\omega^{2}\right)}\left\{\frac{(\lambda-i \omega)\left[\mathrm{e}_{\lambda}(t)\right]^{i \omega}+(\lambda+i \omega)\left[\mathrm{e}_{\lambda}(t)\right]^{-i \omega}}{2}\right\}+C_{1} \\
& \int d t \mathrm{~s}_{\lambda}(\omega, t)=\frac{(1+\lambda t)}{\left(\lambda^{2}+\omega^{2}\right)}\left\{\frac{(\lambda-i \omega)\left[\mathrm{e}_{\lambda}(t)\right]^{i \omega}-(\lambda+i \omega)\left[\mathrm{e}_{\lambda}(t)\right]^{-i \omega}}{2 i}\right\}+C_{2}
\end{aligned}
$$

em que $C_{1}$ e $C_{2}$ não dependem de $t$. Quando $\lambda=0$, reobtém-se as expressões próprias das funções trigonométricas.

Integrando em $\omega$, obtém-se ainda expressões suficientemente simples:

$$
\begin{aligned}
& \int d \omega \mathrm{c}_{\lambda}(\omega, t)=\frac{\mathrm{s}_{\lambda}(\omega, t)}{\ln \left[\mathrm{e}_{\lambda}(t)\right]}+C_{3} \\
& \int d \omega \mathrm{s}_{\lambda}(\omega, t)=-\frac{\mathrm{c}_{\lambda}(\omega, t)}{\ln \left[\mathrm{e}_{\lambda}(t)\right]}+C_{4}
\end{aligned}
$$

em que $C_{3}$ e $C_{4}$ são constantes, que não dependem de $\omega$. Em todos os casos, quando $\lambda \rightarrow 0$, reobtemos as expressões próprias das funções trigonométricas. Segue-se com uma possível aplicação para $c_{\lambda}(\omega, t)$ e $\mathrm{s}_{\lambda}(\omega, t)$.

\subsubsection{Integrais de produtos para série de Fourier generalizada}

Muitas vezes é conveniente escrever uma determinada função em termos de senos e cossenos. Apesar da dedicação de Euler, D. Bernoulli, J.d'Alembert e Lagrange, a decomposição que se mostrou mais consistente foi a apresentada por Fourier, em 1811. A decomposição de funções nas chamadas séries de Fourier foi obtida 
na tentativa de solucionar um problema relativo à propagação do calor em corpos sólidos. Embora esse fosse o objetivo inicial, os estudos das séries de Fourier não ficaram restritos a ele. Foram utilizados também para resolver muitos outros problemas relacionados, principalmente, à equações diferenciais parciais [36].

De modo geral, a série de Fourier de uma função periódica $f(t)$ tal que $f(t+2 L)=f(t)$, com $2 L$ sendo o período, é dada por:

$$
F[f(t)]=\frac{a_{0}}{2}+\sum_{n=1}^{\infty}\left[a_{n} \cos \left(\frac{n \pi t}{L}\right)+b_{n} \operatorname{sen}\left(\frac{n \pi t}{L}\right)\right]
$$

em que $a_{0}, a_{n}$ e $b_{n}$ são dados, respectivamente por

$$
\begin{aligned}
& a_{0}=\frac{1}{L} \int_{c}^{c+2 L} f(t) d t \\
& a_{n}=\frac{1}{L} \int_{c}^{c+2 L} f(t) \cos \left(\frac{n \pi t}{L}\right) d t \\
& b_{n}=\frac{1}{L} \int_{c}^{c+2 L} f(t) \operatorname{sen}\left(\frac{n \pi t}{L}\right) d t,
\end{aligned}
$$

para um valor $c$ qualquer, já que a integração deve ser feita num intervalo de comprimento igual ao período de $f$.

É importante destacar que a definição da série de Fourier é feita mediante funções ortogonais, já que, de fato, para a série usual, decomposta em termos de $\cos \left(\omega_{1} t\right)$ e $\operatorname{sen}\left(\omega_{2} t\right)$, temos que:

$$
\int_{-\infty}^{\infty} \operatorname{sen}\left(\omega_{2} t\right) \cos \left(\omega_{1} t\right) d t=0, \forall \omega_{1}, \omega_{2}, t \in \mathbb{R}
$$

Além disso, outros resultados de integrais que envolvem o produto de $\cos \left(\omega_{1} t\right)$ e sen $\left(\omega_{2} t\right)$ são necessários no processo de decomposição, à saber:

$$
\begin{aligned}
& \int_{-\infty}^{\infty} \operatorname{sen}\left(\omega_{2} t\right) \operatorname{sen}\left(\omega_{1} t\right) d t=\delta\left(\omega_{2}-\omega_{1}\right)+\delta\left(\omega_{2}+\omega_{1}\right) \\
& \int_{-\infty}^{\infty} \cos \left(\omega_{2} t\right) \cos \left(\omega_{1} t\right) d t=\delta\left(\omega_{2}-\omega_{1}\right)-\delta\left(\omega_{2}+\omega_{1}\right),
\end{aligned}
$$

em que $\delta(x-a)$ é a distribuição delta de Dirac.

No contexto de generalizações consistentes, para encontrar uma expressão que generalize as séries de Fourier, mediante a utilização das funções trigonométricas generalizadas, cosseno $\mathrm{c}_{\lambda}\left(\omega_{1}, t\right)$ e seno $\mathrm{s}_{\lambda}\left(\omega_{2}, t\right)$, é necessário que estas funções sejam, obrigatoriamente, ortogonais. Inspirados no mapeamento conforme, $z=\ln \left[\mathrm{e}_{\lambda}(t)\right]$ utilizado na generalização da transformada de Fourier, propomos o peso $(1+\lambda t)^{-1}$ 
(inverso do Jacobiano da transformação) no produto escalar. Assim:

$$
\begin{aligned}
\int_{-\infty}^{\infty} \frac{\mathrm{s}_{\lambda}\left(\omega_{1}, t\right) \mathrm{c}_{\lambda}\left(\omega_{2}, t\right)}{1+\lambda t} d t=0 & \\
\int_{-\infty}^{\infty} \frac{\mathrm{s}_{\lambda}\left(\omega_{1}, t\right) \mathrm{s}_{\lambda}\left(\omega_{2}, t\right)}{1+\lambda t} d t= & \delta\left(\omega_{1}-\omega_{2}\right)\left(1-\mathrm{e}^{-\frac{\pi}{\lambda}\left|\omega_{1}-\omega_{2}\right|}\right)+ \\
& -\delta\left(\omega_{1}+\omega_{2}\right)\left(1-\mathrm{e}^{-\frac{\pi}{\lambda}\left|\omega_{1}+\omega_{2}\right|}\right), \\
\int_{-\infty}^{\infty} \frac{\mathrm{c}_{\lambda}\left(\omega_{1}, t\right) \mathrm{c}_{\lambda}\left(\omega_{2}, t\right)}{1+\lambda t} d t= & \delta\left(\omega_{1}-\omega_{2}\right)\left(1-\mathrm{e}^{-\frac{\pi}{\lambda}\left|\omega_{1}-\omega_{2}\right|}\right)+ \\
& +\delta\left(\omega_{1}+\omega_{2}\right)\left(1-\mathrm{e}^{-\frac{\pi}{\lambda}\left|\omega_{1}+\omega_{2}\right|}\right),
\end{aligned}
$$

Destes resultados podemos concluir que $c_{\lambda}\left(\omega_{1}, t\right)$ e $\mathrm{s}_{\lambda}\left(\omega_{2}, t\right)$ são ortogonais e, além disso, quando $\lambda \rightarrow 0$ retomamos os resultados relativos às funções originais. Este é o primeiro passo para formalização da generalização desta ferramenta, que faz parte das perspectivas futuras. Mesmo assim, fica claro que $c_{\lambda}(\omega, t)$ e $\mathrm{s}_{\lambda}(\omega, t)$ são generalizações consistentes e estendem mais uma característica própria das funções $\cos (t)$ e $\operatorname{sen}(t)$ tradicionais. O Apêndice 2 apresenta as generalizações das demais funções trigonométricas.

Na sequência, apresenta-se uma generalização para as funções hiperbólicas com a mesma ideia aplicada às funções trigonométricas circulares.

\subsubsection{As funções hiperbólicas generalizadas}

A primeira generalização das funções hiperbólicas, dadas por Borges [31], apresentavam $\cosh _{\lambda}(\omega t)$ e $\operatorname{senh}_{\lambda}(\omega t)$ respectivamente como:

$$
\begin{aligned}
\cosh _{\lambda}(\omega t) & =\frac{\mathrm{e}_{\lambda}(\omega t)+\mathrm{e}_{\lambda}(-\omega t)}{2} \\
\operatorname{senh}_{\lambda}(\omega t) & =\frac{\mathrm{e}_{\lambda}(\omega t)-\mathrm{e}_{\lambda}(-\omega t)}{2} .
\end{aligned}
$$

Tal proposta, em seguimento à generalização das funções trigonométricas circulares, relaciona a exponencial tradicional $e^{\omega t}$ à generalizada $e_{\lambda}(\omega t)$. Isso traz como consequência, a dificuldade em validar propriedades e características próprias das versões originais nas formas generalizadas. De forma similar às funções trigonométricas, em que $\left[\mathrm{e}_{\lambda}(t)\right]^{\omega}$ é a extensão de $\mathrm{e}^{\omega t}$, apresentamos uma nova generalização 


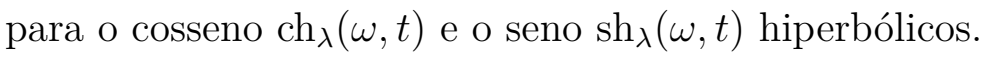

$$
\begin{aligned}
\operatorname{ch}_{\lambda}(\omega, t) & =\frac{\left[\mathrm{e}_{\lambda}(t)\right]^{\omega}+\left[\mathrm{e}_{\lambda}(t)\right]^{-\omega}}{2} \\
\operatorname{sh}_{\lambda}(\omega, t) & =\frac{\left[\mathrm{e}_{\lambda}(t)\right]^{\omega}-\left[\mathrm{e}_{\lambda}(t)\right]^{-\omega}}{2} .
\end{aligned}
$$

A exemplo do que foi estudado para as funções circulares, tem-se também aqui funções de duas variáveis, com $t$ sendo a variável principal e $\omega$ a variável de frequência angular. Nas duas generalizações, reobtém-se as funções originais quando $\lambda \rightarrow 0$.

Essa mudança na interpretação da generalização do exponencial e ${ }^{\omega t}$ traz uma série de vantagens, principalmente para a comparação de características e propriedades que são válidas para as funções originais e passam a valer também para essa extensão. A Fig. 5.8 apresenta o comportamento dessas novas famílias de funções. É interessante observar que tanto na função $\operatorname{ch}_{\lambda}(\omega, t)$ quanto na $\operatorname{sh}_{\lambda}(\omega, t)$, obtém-se curvas semelhantes às das funções originais. Outra observação que se pode fazer acerca da Fig. 5.8 é que todas as curvas aparecem aos pares, exceto a curva em que $\lambda=3$ nas duas funções. Isso ocorre pois existem valores de $t$ para os quais as funções $\operatorname{ch}_{\lambda}(\omega, t)$ e $\operatorname{sh}_{\lambda}(\omega, t)$ não possuem imagem real. De modo geral, são regiões em que $\lambda t<-1$, já que é possível produzir raízes de números negativos, o que proporciona saída complexa. Em alguns casos, pode ser interessante verificar como se comporta a parte real da função. A Fig. 5.9 mostra o comportamento da parte real da função

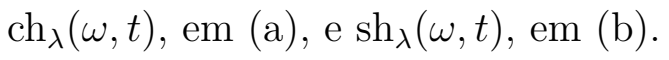

O gráfico da Fig. 5.10 apresenta um exemplo dos efeitos da variável de frequência angular $\omega$ sobre as funções $\operatorname{ch}_{\lambda}(\omega, t)$ e $\operatorname{sh}_{\lambda}(\omega, t)$. Aqui, mantém-se $\lambda=1$ e varia-se $\omega$ com valores 2,4 e 6, respectivamente. Os gráficos da Fig. 5.10 mostram a simetria entre as curvas plotadas. Contudo, não é isso que ocorre para os demais valores de $\lambda$; mantém-se características de funções hiperbólicas porém são um pouco "difusas". Seguem algumas propriedades importantes das funções hiperbólicas que mantém sua validade na versão generalizada.

\subsubsection{Relações iniciais}

A comparação de características e propriedades entre as versões generalizadas e as usuais é um caminho para indicar se determinada expressão é uma generalização consistente. Essa tarefa não deve estar restrita às propriedades mais complicadas 

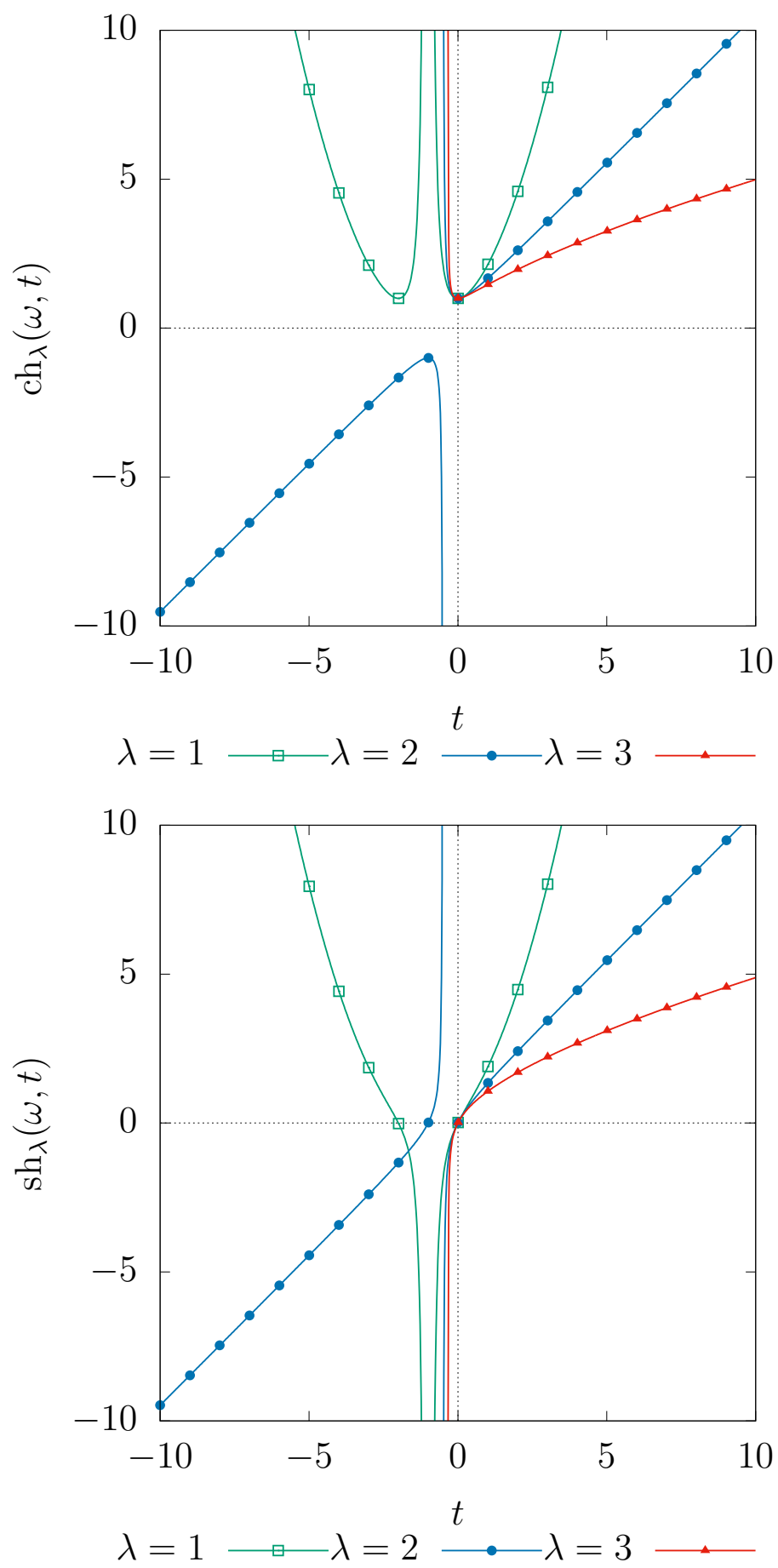

Figura 5.8: (a) Nova generalização para a função cosseno hiperbólico com valores de $\lambda=1, \lambda=2$ e $\lambda=3$, além de $\omega=2$. (b) Nova generalização para a função seno hiperbólico com valores de $\lambda=1, \lambda=2$ e $\lambda=3$, além de $\omega=2$. Em ambos exemplos foram obtidas funções semelhantes a ramos de hipérboles. 

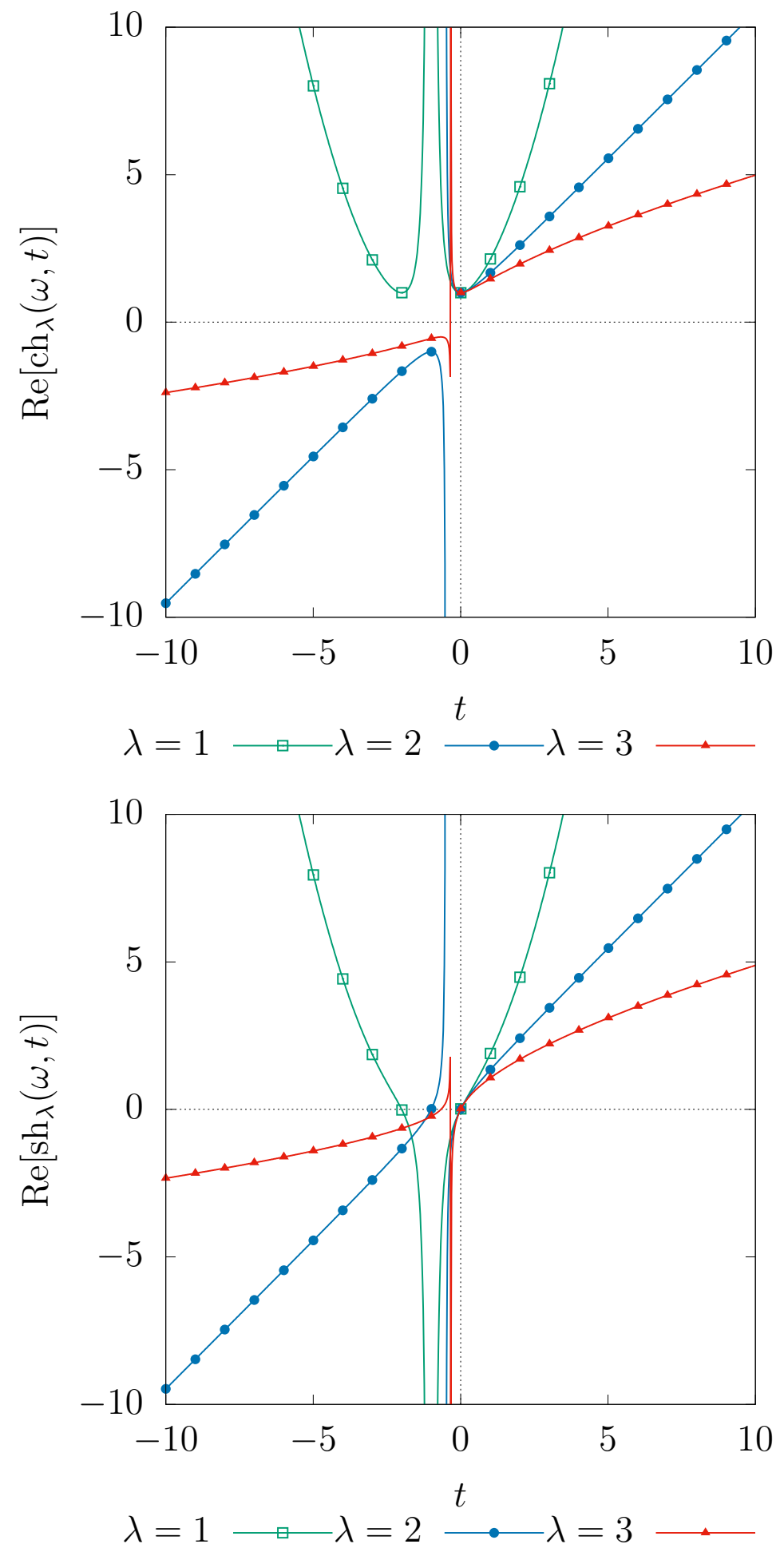

Figura 5.9: (a) Parte real da função $\operatorname{ch}_{\lambda}(\omega, t)$ para os valores de $\lambda=1, \lambda=2$ e $\lambda=3$, além de $\omega=2$. (b) Parte real da função $\operatorname{sh}_{\lambda}(\omega, t)$ para os valores de $\lambda=1, \lambda=2$ e $\lambda=3$, além de $\omega=2$. Em ambos exemplos foram obtidas hipérboles completas. 

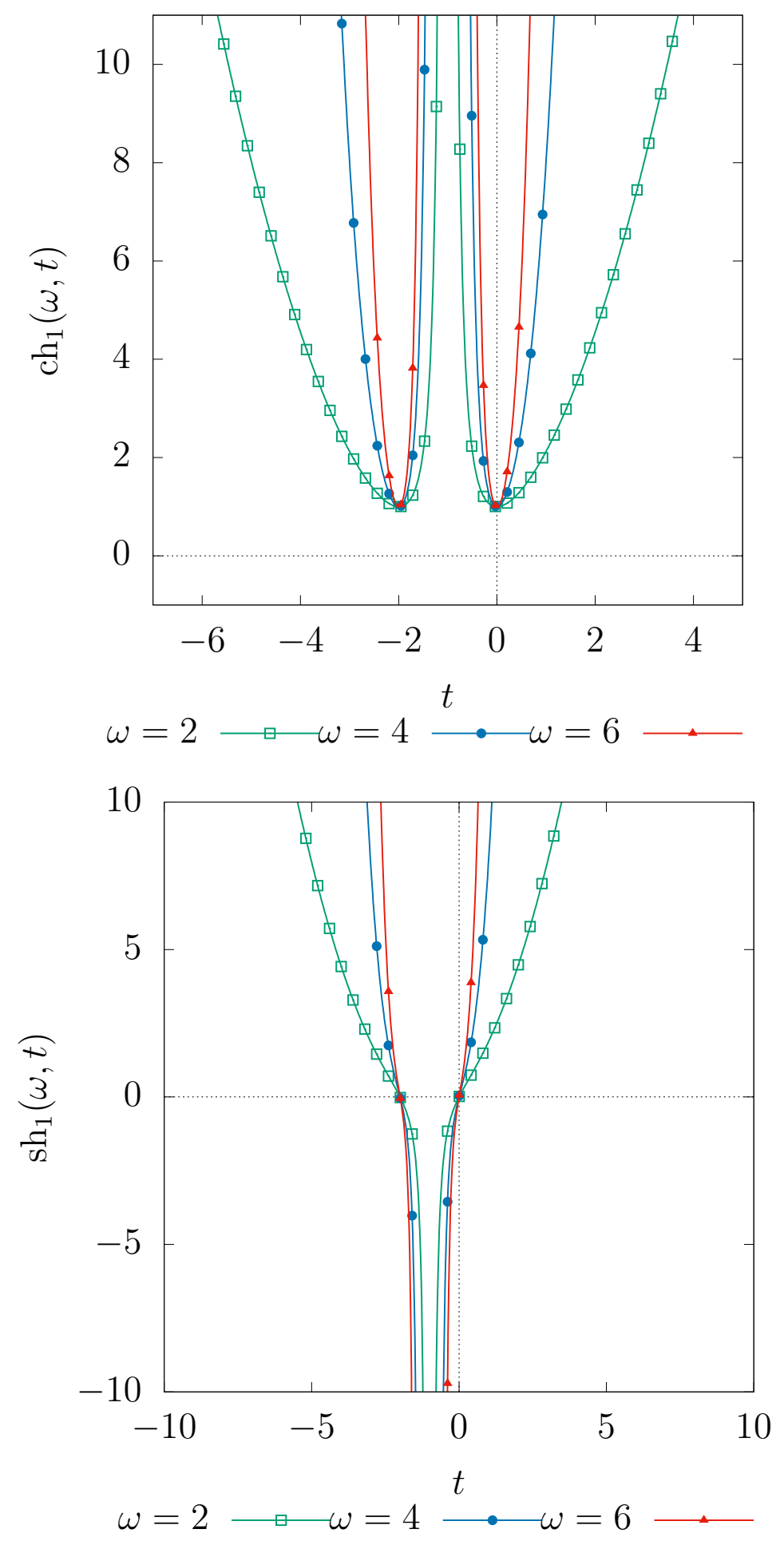

Figura 5.10: (a) Função $\operatorname{ch}_{1}(\omega, t)$ para os valores de $\omega=2, \omega=4$ e $\omega=6$. (b) Função $\operatorname{sh}_{1}(\omega, t)$ para os valores de $\omega=2, \omega=4$ e $\omega=6$. Em ambos exemplos mantém-se fixo $\lambda=1$. 
ou sofisticadas. Entender como pequenas questões afetam o comportamento de toda a família de funções é muito importante. Um bom exemplo é o caso em que $\cosh (\omega t)+\operatorname{senh}(\omega t)=\mathrm{e}^{\omega t}$, para as funções hiperbólicas originais. Ao estender esse resultado para a nossa generalização, verifica-se que

$$
\operatorname{sh}_{\lambda}(\omega, t)+\operatorname{ch}_{\lambda}(\omega, t)=\left[\mathrm{e}_{\lambda}(t)\right]^{\omega} .
$$

De forma similar, sendo $\operatorname{senh}(\omega t)-\cosh (\omega t)=-\mathrm{e}^{-\omega t}$ válido para as funções tradicionais, tem-se, na versão generalizada

$$
\operatorname{sh}_{\lambda}(\omega, t)-\operatorname{ch}_{\lambda}(\omega, t)=-\left[\mathrm{e}_{\lambda}(t)\right]^{-\omega}
$$

Tais igualdades valem também nas generalizações propostas por Borges [31]. Outra importante relação válida para as funções tradicionais é

$$
[\operatorname{senh}(\omega t)+\cosh (\omega t)]^{n}=\left[\mathrm{e}^{\omega t}\right]^{n}=\mathrm{e}^{n \omega t}=\operatorname{senh}(n \omega t)+\cosh (n \omega t) .
$$

Na generalização proposta a relação equivalente é dada por

$$
\begin{aligned}
{\left[\operatorname{sh}_{\lambda}(\omega, t)+\operatorname{ch}_{\lambda}(\omega, t)\right]^{n} } & =\left[\mathrm{e}_{\lambda}(t)^{\omega}\right]^{n}=\left[\mathrm{e}_{\lambda}(t)^{n}\right]^{\omega}= \\
& =\left[\mathrm{e}_{\lambda / n}(n t)\right]^{\omega}=\operatorname{sh}_{\lambda / n}(\omega, n t)+\operatorname{ch}_{\lambda / n}(\omega, n t) .
\end{aligned}
$$

Desta forma, vê-se que a proposta de generalização apresentada aqui acompanha resultados simples, já que quando $\lambda \rightarrow 0$ retornamos às propriedades originais, o que não é observado na generalização anterior.

\subsubsection{Relação fundamental}

Assim como no caso das funções circulares, as hiperbólicas também apresentam uma generalização eficaz para a relação fundamental. É conhecido que para as funções tradicionais vale a relação fundamental $\cosh ^{2}(\omega t)-\operatorname{senh}^{2}(\omega t)=1$. Em nossa generalização, essa relação é estendida a

$$
\operatorname{ch}_{\lambda}^{2}(\omega, t)-\operatorname{sh}_{\lambda}^{2}(\omega, t)=1 \quad \forall \lambda, \omega, t,
$$

diferente da generalização anterior, onde se obtém

$$
\cosh _{\lambda}^{2}(\omega t)-\operatorname{senh}_{\lambda}^{2}(\omega t)=\mathrm{e}_{\lambda}\left(-\lambda \omega^{2} t^{2}\right)
$$

A Eq. (5.84) tem valor unitário apenas quando $\lambda \rightarrow 0$ e, a exemplo do que ocorre na relação fundamental da trigonometria, soa um tanto quanto estranho, já que 
tal relação tem origem em relações puramente geométricas. Nestes termos, nossa generalização se mostra mais consistente já que tem valor unitário para quaisquer valores reais de $\lambda, \omega$ e $t$. Na sequência, apresenta-se outras propriedades das funções hiperbólicas.

\subsubsection{Produtos}

A exemplo das funções circulares, as hiperbólicas também possuem resultados que podem auxiliar em cálculos e simplificar expressões. A transformação de produtos em somas é uma ferramenta muito útil às funções tradicionais. Na nossa proposta, consegue-se essa relação usando os operadores soma e diferença generalizados, da seguinte forma:

$$
\begin{aligned}
\operatorname{sh}_{\lambda}(\omega, a) \operatorname{ch}_{\lambda}(\omega, b) & =\frac{1}{2}\left[\operatorname{sh}_{\lambda}\left(\omega, a \oplus_{\lambda} b\right)-\operatorname{sh}_{\lambda}\left(\omega, a \ominus_{\lambda} b\right)\right] ; \\
\operatorname{sh}_{\lambda}(\omega, a) \operatorname{sh}_{\lambda}(\omega, b) & =\frac{1}{2}\left[\operatorname{ch}_{\lambda}\left(\omega, a \oplus_{\lambda} b\right)-\operatorname{ch}_{\lambda}\left(\omega, a \ominus_{\lambda} b\right)\right] ; \\
\operatorname{ch}_{\lambda}(\omega, a) \operatorname{ch}_{\lambda}(\omega, b) & =\frac{1}{2}\left[\operatorname{ch}_{\lambda}\left(\omega, a \oplus_{\lambda} b\right)+\operatorname{ch}_{\lambda}\left(\omega, a \ominus_{\lambda} b\right)\right],
\end{aligned}
$$

em que $\oplus_{\lambda}$ e $\ominus_{\lambda}$ são, respectivamente, a soma e a diferença generalizada apresentadas no Cap. 2. Quando $\lambda \rightarrow 0$, retomamos a propriedade para as funções hiperbólicas tradicionais. Relação parecida pode ser obtida para a variável de frequência angular $\omega$ sem uso dos operadores generalizados:

$$
\begin{aligned}
\operatorname{sh}_{\lambda}\left(\omega_{1}, t\right) \operatorname{ch}_{\lambda}\left(\omega_{2}, t\right) & =\frac{1}{2}\left[\operatorname{sh}_{\lambda}\left(\omega_{1}+\omega_{2}, t\right)+\operatorname{sh}_{\lambda}\left(\omega_{1}-\omega_{2}, t\right)\right] \\
\operatorname{sh}_{\lambda}\left(\omega_{1}, t\right) \operatorname{sh}_{\lambda}\left(\omega_{2}, t\right) & =\frac{1}{2}\left[\operatorname{ch}_{\lambda}\left(\omega_{1}+\omega_{2}, t\right)-\operatorname{ch}_{\lambda}\left(\omega_{1}-\omega_{2}, t\right)\right] \\
\operatorname{ch}_{\lambda}\left(\omega_{1}, t\right) \operatorname{ch}_{\lambda}\left(\omega_{2}, t\right) & =\frac{1}{2}\left[\operatorname{ch}_{\lambda}\left(\omega_{1}+\omega_{2}, t\right)+\operatorname{ch}_{\lambda}\left(\omega_{1}-\omega_{2}, t\right)\right] .
\end{aligned}
$$

Obtém-se, desta forma, uma propriedade para a função generalizada equivalente à tradicional.

\subsubsection{Derivadas}

No campo da análise matemática, estudando de forma específica as derivadas de nossa proposta de generalização, verifica-se seguintes expressões para $\operatorname{ch}_{\lambda}(\omega, t)$ e 
$\operatorname{sh}_{\lambda}(\omega, t):$

$$
\begin{aligned}
& \frac{d^{n} \operatorname{ch}_{\lambda}(\omega, t)}{d t^{n}}=\eta(t) \sum_{\ell=1}^{n} \frac{\omega^{2 \ell-1}}{\lambda^{2 \ell-n}}\left[\lambda \mathbb{S}(n, 2 \ell-1) \operatorname{sh}_{\lambda}(\omega, t)+\omega \mathbb{S}(n, 2 \ell) \operatorname{ch}_{\lambda}(\omega, t)\right] \\
& \frac{d^{n} \operatorname{sh}_{\lambda}(\omega, t)}{d t^{n}}=\eta(t) \sum_{\ell=1}^{n} \frac{\omega^{2 \ell-1}}{\lambda^{2 \ell-n}}\left[\lambda \mathbb{S}(n, 2 \ell-1) \operatorname{ch}_{\lambda}(\omega, t)+\omega \mathbb{S}(n, 2 \ell) \operatorname{sh}_{\lambda}(\omega, t)\right]
\end{aligned}
$$

em que $\eta(t)=1 /(1+\lambda t)^{n}$ e $\mathbb{S}(n, m)$, à exemplo das funções circulares, se refere ao $m$-ésimo número de Stirling de primeiro tipo e ordem $n$.

Ao mudar-se a variável de derivação para a frequência angular $\omega$, as seguintes expressões são obtidas para as derivadas de $\operatorname{ch}_{\lambda}(\omega, t)$ e $\operatorname{sh}_{\lambda}(\omega, t)$ respectivamente:

$$
\begin{aligned}
& \frac{d^{n} \operatorname{ch}_{\lambda}(\omega, t)}{d \omega^{n}}=\left[\ln \left(\mathrm{e}_{\lambda}(t)\right)\right]^{n} H_{n}(\omega, t) \\
& \frac{d^{n} \operatorname{sh}_{\lambda}(\omega, t)}{d \omega^{n}}=\left[\ln \left(\mathrm{e}_{\lambda}(t)\right)\right]^{n} H_{n+1}(\omega, t),
\end{aligned}
$$

em que

$$
H_{k}(\omega, t)=\left\{\begin{array}{rr}
\operatorname{sh}_{\lambda}(\omega, t) & \text { se } k \text { é par } \\
\operatorname{ch}_{\lambda}(\omega, t) & \text { se } k \text { é impar }
\end{array}\right.
$$

Derivando no parâmetro $\lambda$, as expressões obtidas para $\operatorname{ch}_{\lambda}(\omega, t)$ e $\operatorname{sh}_{\lambda}(\omega, t)$ são, respectivamente:

$$
\begin{aligned}
\frac{d}{d \lambda} \operatorname{ch}_{\lambda}(\omega, t) & =\frac{\omega}{\lambda}\left[\frac{t}{1+\lambda t}-\ln \left[\mathrm{e}_{\lambda}(t)\right]\right] \operatorname{sh}_{\lambda}(\omega, t) \\
\frac{d}{d \lambda} \operatorname{ch}_{\lambda}(\omega, t) & =\frac{\omega}{\lambda}\left[\frac{t}{1+\lambda t}-\ln \left[\mathrm{e}_{\lambda}(t)\right]\right] \operatorname{ch}_{\lambda}(\omega, t) .
\end{aligned}
$$

Estes resultados mostram uma conexão entre as propostas de generalização das funções circulares e hiperbólicas, pelo fator que multiplica ambas, após derivação no parâmetro $\lambda$. A validade de propriedades e características das funções hiperbólicas em suas generalizações são fortes indícios de que nossa generalização está contida no seleto conjunto das generalizações consistentes! Seguem mais algumas características importantes, relativas ao cálculo integral.

\subsubsection{Integração}

A integração das funções hiperbólicas tradicionais é relativamente simples: integrando o cosseno hiperbólico obtém-se o seno hiperbólico e integrando o seno 
obtém-se o cosseno. Nesta generalização, obtém-se os seguintes resultados:

$$
\begin{aligned}
& \int \operatorname{ch}_{\lambda}(\omega, t) d t=\frac{(1+\lambda t)}{2\left(\lambda^{2}-\omega^{2}\right)}\left\{(\lambda-\omega)\left[\mathrm{e}_{\lambda}(t)\right]^{\omega}+(\lambda+\omega)\left[\mathrm{e}_{\lambda}(t)\right]^{-\omega}\right\} ; \\
& \int \operatorname{sh}_{\lambda}(\omega, t) d t=\frac{(1+\lambda t)}{2\left(\lambda^{2}-\omega^{2}\right)}\left\{(\lambda-\omega)\left[\mathrm{e}_{\lambda}(t)\right]^{\omega}-(\lambda+\omega)\left[\mathrm{e}_{\lambda}(t)\right]^{-\omega}\right\}
\end{aligned}
$$

que quando $\lambda=0$, retorna os resultados esperados de $\operatorname{senh}(\omega t)$ e $\cosh (\omega t)$, respectivamente.

Ainda quanto à integração, ao estudar a variável de frequência angular $\omega$, obtém-se expressões mais simples,

$$
\begin{aligned}
\int \operatorname{ch}_{\lambda}(\omega, t) d \omega & =\frac{\operatorname{sh}_{\lambda}(\omega, t)}{\ln \left(\mathrm{e}_{\lambda}(t)\right)}, \\
\int \operatorname{sh}_{\lambda}(\omega, t) d \omega & =\frac{\operatorname{ch}_{\lambda}(\omega, t)}{\ln \left(\mathrm{e}_{\lambda}(t)\right)} .
\end{aligned}
$$

Desta forma, verifica-se grande compatibilidade desta proposta de generalização com as funções originais, no que tange o cálculo diferencial e integral. Mais algumas propriedades tornam ainda mais forte essa proposta.

\subsubsection{Relacionando as funções seno e cosseno generalizadas com as fun- ções hiperbólicas generalizadas}

Da trigonometria tradicional, pode-se constatar uma relação bem simples entre as funções circulares e hiperbólicas. Espera-se que, em uma generalização consistente, tal relação seja mantida. De fato, na proposta de generalização obtémse que:

$$
\begin{aligned}
\mathrm{c}_{\lambda}(\omega, i t) & =\operatorname{ch}_{i \lambda}(\omega, t) ; \\
\operatorname{ch}_{\lambda}(\omega, i t) & =\mathrm{c}_{i \lambda}(\omega, t) ; \\
\mathrm{s}_{\lambda}(\omega, i t) & =i \operatorname{sh}_{i \lambda}(\omega, t) \\
\operatorname{sh}_{\lambda}(\omega, i t) & =i \mathrm{~s}_{i \lambda}(\omega, t) .
\end{aligned}
$$

Novamente, quando $\lambda \rightarrow 0$, reobtém-se as relações originais.

Outra maneira de se obter uma relação entre as funções circulares e hiperbólicas generalizadas na variável $t$ é utilizar a forma log-periódica da função $c_{\lambda}(\omega, t)$. Levando-se em conta que a função $\ln _{\lambda}(t)$ é a inversa de $e_{\lambda}(t)$ e que $c_{\lambda}(\omega, t)=\cos \left\{\omega \ln \left[\mathrm{e}_{\lambda}(t)\right]\right\}$, segue-se que

$$
c_{\lambda}\left[\omega, \ln _{\lambda}(t)\right]=\cos [\omega \ln (t)] .
$$


Aqui é obtida uma outra generalização para as funções log-periódicas, utilizando agora a função logaritmo natural generalizado. Pela relação das funções hiperbólicas e trigonométricas, obtém-se $c_{\lambda}\left[\omega, \ln _{\lambda}(t)\right]=\cos [\omega \ln (t)]=\cosh [i \omega \ln (t)]$. Por outro lado, $\operatorname{como} \operatorname{ch}_{\lambda}(\omega, t)=\cosh \left\{\omega \ln \left[\mathrm{e}_{\lambda}(t)\right]\right\}$, segue que $\operatorname{ch}_{\lambda}\left[\omega, \ln _{\lambda}(t)\right]=\cosh [\omega \ln (t)]=$ $\cos [i \omega \ln (t)]=\cos \left[\omega \ln \left(t^{i}\right)\right]=c_{\lambda}\left[\omega, \ln _{\lambda}\left(t^{i}\right)\right]$. À partir daí, seguem as relações:

$$
\begin{aligned}
\mathrm{c}_{\lambda}\left(\omega, \ln _{\lambda}(t)\right) & =\operatorname{ch}_{\lambda}\left(\omega, \ln _{\lambda}\left(t^{i}\right)\right) ; \\
\operatorname{ch}_{\lambda}\left(\omega, \ln _{\lambda}(t)\right) & =\mathrm{c}_{\lambda}\left(\omega, \ln _{\lambda}\left(t^{i}\right)\right) ; \\
\mathrm{s}_{\lambda}\left(\omega, \ln _{\lambda}(t)\right) & =-i \operatorname{sh}_{\lambda}\left(\omega, \ln _{\lambda}\left(t^{i}\right)\right) ; \\
\operatorname{sh}_{\lambda}\left(\omega, \ln _{\lambda}(t)\right) & =-i \mathrm{~s}_{\lambda}\left(\omega, \ln _{\lambda}\left(t^{i}\right)\right)
\end{aligned}
$$

Obtemos, desta forma, além das relações entre as generalizações das funções trigonométricas com as hiperbólicas, outras generalizações para as funções logperiódicas, utilizando a função $\ln _{\lambda}(t)$.

Existe ainda, nesta generalização, uma relação entre as funções hiperbólicas e circulares no que diz respeito à variável $\omega$. Desta forma,

$$
\begin{aligned}
c_{\lambda}(i \omega, t) & =\operatorname{ch}_{\lambda}(\omega, t) ; \\
\operatorname{ch}_{\lambda}(i \omega, t) & =\mathrm{c}_{\lambda}(\omega, t) ; \\
\mathrm{s}_{\lambda}(i \omega, t) & =i \operatorname{sh}_{\lambda}(\omega, t) ; \\
\operatorname{sh}_{\lambda}(i \omega, t) & =i \mathrm{~s}_{\lambda}(\omega, t) .
\end{aligned}
$$

Verifica-se, portanto, que a proposta apresentada para generalização das funções hiperbólicas e circulares é muito consistente e, ao mesmo tempo, generaliza também às funções log-periódicas. Desta forma, aplicações que permeiem os conceitos de funções log-periódicas podem utilizar propriedades, simplificações e características inerentes às funções trigonométricas e hiperbólicas generalizadas, e vice-versa. Mais que indicar o fato de encontrar generalizações consistentes para todas essas famílias de funções, mostramos uma conexão coerente entre todos esses assuntos.

Vale lembrar que, para se obter a generalização para as demais funções trigonométricas e hiperbólicas, basta utilizar relações equivalentes àquelas válidas para as funções usuais. Desta forma, para encontrar, por exemplo, a função tangente generalizada, $t_{\lambda}(\omega, t)$, basta fazer a razão entre as funções $s_{\lambda}(\omega, t)$ e $c_{\lambda}(\omega, t)$. Assim, 


$$
\mathrm{t}_{\lambda}(\omega, t)=\frac{\mathrm{s}_{\lambda}(\omega, t)}{\mathrm{c}_{\lambda}(\omega, t)} .
$$

Processo semelhante generaliza também as demais funções trigonométricas e hiperbólicas. O Apêndice 2 apresenta, de modo simplificado, as generalizações das demais funções trigonométricas e hiperbólicas, bem como exemplos de curvas pertencentes a cada uma destas famílias de funções.

\subsubsection{Exemplo e conclusões}

Para verificar a consistência de nossas generalizações, encerra-se esta discussão com um exemplo que faz uso da generalização da transformada de Fourier apresentada no Cap. 4 como:

$$
\mathcal{F}_{\lambda}[f(t)]=\frac{1}{\sqrt{4 \pi}} \int_{-\infty}^{\infty} \frac{\left[\mathrm{e}_{\lambda}(t)\right]^{i \omega}}{|1+\lambda t|} f(t) d t .
$$

Neste exemplo, toma-se $f(t)=c_{\lambda}\left(\omega_{0}, t\right)$, o que resulta em

$$
\mathcal{F}_{\lambda}\left[\mathrm{c}_{\lambda}\left(\omega_{0}, t\right)\right]=\frac{1}{\sqrt{4 \pi}} \int_{-\infty}^{\infty} \frac{\left[\mathrm{e}_{\lambda}(t)\right]^{i \omega}}{|1+\lambda t|} \mathrm{c}_{\lambda}\left(\omega_{0}, t\right) d t
$$

Substituindo $c_{\lambda}\left(\omega_{0}, t\right)$ por sua definição

$$
\mathrm{c}_{\lambda}\left(\omega_{0}, t\right)=\frac{\left[\mathrm{e}_{\lambda}(t)\right]^{i \omega_{0}}+\left[\mathrm{e}_{\lambda}(t)\right]^{-i \omega_{0}}}{2},
$$

a Eq. (5.99) pode ser reescrita como:

$$
\mathcal{F}_{\lambda}\left[\mathrm{c}_{\lambda}\left(\omega_{0}, t\right)\right]=\frac{1}{\sqrt{4 \pi}} \int_{-\infty}^{\infty} \frac{\left[\mathrm{e}_{\lambda}(t)\right]^{i \omega}}{|1+\lambda t|} \frac{\left[\mathrm{e}_{\lambda}(t)\right]^{i \omega_{0}}+\left[\mathrm{e}_{\lambda}(t)\right]^{-i \omega_{0}}}{2} d t .
$$

Aplicando a propriedade distributiva, chega-se ao seguinte resultado:

$$
\mathcal{F}_{\lambda}\left[\mathrm{c}_{\lambda}\left(\omega_{0}, t\right)\right]=\frac{1}{\sqrt{4 \pi}} \int_{-\infty}^{\infty} \frac{1}{2}\left\{\frac{\left[\mathrm{e}_{\lambda}(t)\right]^{i \omega}}{|1+\lambda t|}\left[\mathrm{e}_{\lambda}(t)\right]^{i \omega_{0}}+\frac{\left[\mathrm{e}_{\lambda}(t)\right]^{i \omega}}{|1+\lambda t|}\left[\mathrm{e}_{\lambda}(t)\right]^{-i \omega_{0}} d t\right\} .
$$

Separando em duas integrais,

$$
\mathcal{F}_{\lambda}\left[\mathrm{c}_{\lambda}\left(\omega_{0}, t\right)\right]=\frac{1}{\sqrt{4 \pi}}\left\{\frac{1}{2} \int_{-\infty}^{\infty} \frac{\left[\mathrm{e}_{\lambda}(t)\right]^{i\left(\omega-\omega_{0}\right)}}{|1+\lambda t|} d t+\frac{1}{2} \int_{-\infty}^{\infty} \frac{\left[\mathrm{e}_{\lambda}(t)\right]^{i\left(\omega-\omega_{0}\right)}}{|1+\lambda t|} d t\right\} .
$$

Cada uma das integrais obtidas são novas transformadas de Fourier generalizadas da função $f(t)=1$. Deste modo, conforme já mostrado no exemplo da Eq. (4.31) do Cap. 4, temos que

$$
\mathcal{F}_{\lambda}\left[c_{\lambda}\left(\omega_{0}, t\right)\right]=\sqrt{\pi}\left[\delta_{\lambda}\left(\omega+\omega_{0}\right)+\delta_{\lambda}\left(\omega-\omega_{0}\right)\right]
$$


em que $\delta(t)$ é a distribuição delta de Dirac.

No contexto das generalizações consistentes, verifica-se que, portanto, nossa proposta para as funções trigonométricas, hiperbólicas e log-periódicas cumprem bem o seu papel. Graças a nova proposta de generalização da função exponencial $\mathrm{e}_{\lambda}(t)$, que leva $\mathrm{e}^{i \omega t}$ em $\left[\mathrm{e}_{\lambda}(t)\right]^{i \omega}$, foi possível encontrar expressões que preservam características, propriedades e aplicabilidade. De fato, os conteúdos apresentados aqui, podem se converter em ferramentas eficazes ao estudo de fraturas de materiais, mercado financeiro, transformação de dados, entre outros. 


\section{6}

\section{Conclusão e Perspectivas}

Em algum lugar, alguma coisa incrível está esperando para ser descoberta.

- Carl Sagan

As generalizações mais consistentes são aquelas que melhor preservam características, propriedades e sustentam a aplicabilidade das funções originais a sistemas mais realistas. Nesta tese, apresentamos uma nova formulação para a função exponencial generalizada

$$
\mathrm{e}_{\lambda}(t)=\lim _{\lambda^{\prime} \rightarrow \lambda}\left|1+\lambda^{\prime} t\right|^{\frac{1}{\lambda^{\prime}}} \mathrm{e}^{i \frac{\pi}{\lambda^{\prime}}\left[1-\theta\left(1+\lambda^{\prime} t\right)\right]},
$$

que, a exemplo da exponencial tradicional, é analítica no plano complexo. Analisamos a generalização de Tsallis e verificamos que a imposição, $e_{\lambda}(t)=0$ se $\lambda t<-1$ garante, por um lado, que o conjunto imagem da função $e_{\lambda}(t)$ esteja sempre contido no corpo dos números reais, e restringe, por outro lado, a abrangência da generalização. Além disso, ao estendermos a definição original para o plano complexo, mediante a simples troca de $t \in \mathbb{R}$ para $z \in \mathbb{C}$, não conseguimos manter a analiticidade obtida em nossa proposta.

O ganho obtido com nossa nova formulação de $e_{\lambda}(t)$ é visível na generalização de outras estruturas. Ao utilizá-la nas generalizações das funções trigonométricas, hiperbólicas e log-periódicas ou na $\lambda$-TF, verificamos que o resultado são generalizações muito consistentes que preservam várias características, propriedades e aplicabilidade.

Nas generalizações propostas por Tsallis [2], verificamos aplicações em diversas áreas da ciência, entre elas, no contexto das transformações baseadas em Box- 
Cox. Desta maneira, fez-se necessário a utilização dos operadores soma e produto generalizados. Fizemos a análise dessas generalizações, verificamos propriedades válidas e explicitamos que a estrutura algébrica $\left(\mathbb{R}_{\lambda}, \oplus_{\lambda}, \odot_{\lambda}\right)$ é, no mínimo, um anel de integridade.

A generalização da transformada de Fourier passa pela escolha adequada do seu núcleo. Desta forma, encontrar a versão generalizada para a expressão e ${ }^{i \omega t}$ é imperativo. Neste aspecto, revisitamos nossa generalização da transformada de Fourier, apresentada na Ref. [23] e verificamos que seu núcleo, $\left[\mathrm{e}_{\lambda}(t)\right]^{i \omega}$, é compatível com a extensão, para o plano complexo, de $e_{\lambda}(t)$ que propusemos no Cap. 2. De fato, foi verificado que a utilização deste núcleo, associada ao produto convencional, ao invés do generalizado, preservou características e propriedades da transformada original, sendo, inclusive, possível a utilização de um mapa conforme que relaciona diretamente nossa generalização com a transformada usual. A principal característica preservada foi a linearidade, já que, desta forma, para qualquer valor do parâmetro $\lambda$, a $\lambda$-TF é uma transformada integral. A aplicabilidade da transformada de Fourier foi estendida nesta generalização: conseguimos escrever a distribuição delta de Dirac em termos da $\lambda$-TF. Além disso, a nossa generalização da transformada de Fourier parece ter aplicabilidade natural diante de um sinal log-periódico, já que, ao transformar $f(t)=\cos \left[\omega_{0} \ln (1+t)\right]$ ela consegue destacar a significância de $\omega_{0}$ para esse sinal.

A partir deste núcleo, foi possível ainda apresentar, novas generalizações para as funções trigonométricas. Estas, ao contrário das primeiras definições encontradas na literatura [31], preservam as principais características de funções trigonométricas: são limitadas e oscilam. Por conta dessa estrutura, foi possível também verificar e estender, de maneira correta, muitas propriedades das funções tradicionais para as generalizadas, como por exemplo, a relação fundamental da trigonometria. No mesmo espírito, foi possível generalizar também as funções hiperbólicas.

As funções trigonométricas generalizadas encontradas aqui possuem grande aplicabilidade, já que podem ser convertidas em funções log-periódicas generalizadas. De fato, $c_{\lambda}\left[\omega, \ln _{\lambda}(t)\right]=\cos [\omega \ln (t)]$, a função $\log$-periódica utilizada em estudos relativos a fratura de materiais, ativos financeiros, entre outros. A extensão analítica da função exponencial generalizada para o plano complexo permitiu a proposta 
destas novas generalizações e abriu espaço para novas perguntas e investigações.

A primeira nova linha de investigação é na definição de uma nova transformada de Fourier generalizada. Pode-se mapear a nossa generalização na transformada original de modo a preservar suas propriedades, inversa e linearidade, por exemplo. No entanto, ainda devemos estudar em detalhes a transformação das Gaussianas generalizadas que têm extensiva aplicabilidade [63, 86-89].

A segunda nova linha de investigação é sobre a conveniência de definir novas funções generalizadas para as funções trigonométricas e hiperbólicas. Na nossa proposta de generalização, conseguimos preservar muitas das propriedades das funções originais, como a relação fundamental da trigonometria. A nossa proposta de funções trigonométricas generalizadas é totalmente compatível com a nossa proposta da transformada de Fourier. Devemos ainda estudar em detalhes as séries de Fourier usando essas funções trigonométricas generalizadas.

Comum no contexto da transformada de Fourier generalizada e na generalização das funções trigonométricas, são as funções log-periódicas,(que definem as funções trigonométricas) [80, 90]. Estudaremos no futuro dados log-periódicos no contexto das $\lambda$-TF.

Enfim, estaremos abertos a novas descobertas. Seguindo um pensamento de Einstein: "Se, a princípio, a ideia não é absurda, então não há esperança para ela". 


\section{Apêndice}

\section{Derivada de $\mathbf{e}_{\lambda}(t)$ em relação a $\lambda$.}

Considerando a parte real função exponencial generalizada $e_{\lambda}(t)$, tem-se:

$$
\mathrm{e}_{\lambda}(t)=(1+\lambda t)^{\frac{1}{\lambda}}
$$

Ao derivar em relação a $t$, obtém-se a igualdade:

$$
\frac{d}{d t} \mathrm{e}_{\lambda}(t)=\left(\frac{1}{1+\lambda t}\right) \mathrm{e}_{\lambda}(t)
$$

que pode ser reescrita como

$$
\left(\frac{1}{\mathrm{e}_{\lambda}(t)}\right)\left[\frac{d}{d t} \mathrm{e}_{\lambda}(t)\right]=\frac{1}{1+\lambda t} .
$$

O lado esquerdo da Eq. (1.4) é a derivada do logaritmo natural de $\mathrm{e}_{\lambda}(t)$, desta forma

$$
\frac{d}{d t} \ln \left[\mathrm{e}_{\lambda}(t)\right]=\frac{1}{1+\lambda t} .
$$

Ao derivar em relação a $\lambda$ dos dois lados tem-se:

$$
\frac{d}{d \lambda} \frac{d}{d t} \ln \left[\mathrm{e}_{\lambda}(t)\right]=\frac{d}{d \lambda} \frac{1}{1+\lambda t}=(-1) \frac{t}{(1+\lambda t)^{2}},
$$

que, ao mudar a ordem de derivação obtém-se

$$
\frac{d}{d t} \frac{d}{d \lambda} \ln \left[\mathrm{e}_{\lambda}(t)\right]=(-1) \frac{t}{(1+\lambda t)^{2}} .
$$

Derivando sucessivamente em relação a $\lambda$,

$$
\begin{aligned}
& \frac{d}{d t} \frac{d^{2}}{d \lambda^{2}} \ln \left[\mathrm{e}_{\lambda}(t)\right]=(+1) \cdot 2 \cdot \frac{t^{2}}{(1+\lambda t)^{3}}=(-1)^{2} \cdot 2 ! \frac{t^{2}}{(1+\lambda t)^{3}} \\
& \frac{d}{d t} \frac{d^{3}}{d \lambda^{3}} \ln \left[\mathrm{e}_{\lambda}(t)\right]=(-1) \cdot 6 \cdot \frac{t^{3}}{(1+\lambda t)^{4}}=(-1)^{3} \cdot 3 ! \frac{t^{3}}{(1+\lambda t)^{4}}
\end{aligned}
$$




$$
\begin{aligned}
\frac{d}{d t} \frac{d^{4}}{d \lambda^{4}} \ln \left[\mathrm{e}_{\lambda}(t)\right] & =(+1) \cdot 24 \cdot \frac{t^{4}}{(1+\lambda t)^{5}}=(-1)^{4} \cdot 4 ! \frac{t^{4}}{(1+\lambda t)^{5}} \\
\frac{d}{d t} \frac{d^{5}}{d \lambda^{5}} \ln \left[\mathrm{e}_{\lambda}(t)\right] & =(-1) \cdot 120 \cdot \frac{t^{5}}{(1+\lambda t)^{6}}=(-1)^{5} \cdot 4 ! \frac{t^{5}}{(1+\lambda t)^{6}} \\
\vdots & \\
\frac{d}{d t} \frac{d^{n}}{d \lambda^{n}} \ln \left[\mathrm{e}_{\lambda}(t)\right] & =(-1)^{n} \cdot n ! \frac{t^{n}}{(1+\lambda t)^{n+1}} .
\end{aligned}
$$

Ao integrar em $t$, os dois lados da Eq. (1.8), o resultado obtido é:

$$
\frac{d^{n}}{d \lambda^{n}} \ln \left[\mathrm{e}_{\lambda}(t)\right]=(-1)^{n} \cdot n ! \int \frac{t^{n}}{(1+\lambda t)^{n+1}} d t
$$

Na sequência, é necessário calcular a integral em $t$ do lado direito da Eq. (1.9). Para tanto utiliza-se o método de integração por partes, em que

$$
\int u d v=u v-\int v d u \text {. }
$$

Fazendo $u=t^{n}$ tem-se que $d u=n t^{n-1}$. Por outro lado, se $d v=1 /(1+\lambda t)^{n+1}$ então $v=-1 /\left[\lambda n(1+\lambda t)^{n+1}\right]$. Assim,

$$
\int \frac{t^{n}}{(1+\lambda t)^{n+1}} d t=\frac{-t^{n}}{\lambda n(1+\lambda t)^{n}}+\int \frac{t^{n-1}}{\lambda(1+\lambda t)^{n}} d t=\frac{-1}{\lambda n}\left(\frac{t}{1+\lambda t}\right)^{n}+\frac{1}{\lambda} \int \frac{t^{n-1}}{(1+\lambda t)^{n}} d t .
$$

Aplicando uma segunda vez o método de integração por partes, após as devidas simplificações, obtém-se

$$
\int \frac{t^{n}}{(1+\lambda t)^{n+1}} d t=\frac{-1}{\lambda n}\left(\frac{t}{1+\lambda t}\right)^{n}-\frac{1}{\lambda^{2}(n-1)}\left(\frac{t}{1+\lambda t}\right)^{n-1}+\frac{1}{\lambda^{2}} \int \frac{t^{n-2}}{(1+\lambda t)^{n-1}} d t .
$$

Uma terceira aplicação deste método retorna

$$
\begin{aligned}
\int \frac{t^{n}}{(1+\lambda t)^{n+1}} d t=\frac{-1}{\lambda n}\left(\frac{t}{1+\lambda t}\right)^{n} & -\frac{1}{\lambda^{2}(n-1)}\left(\frac{t}{1+\lambda t}\right)^{n-1}-\frac{1}{\lambda^{3}(n-2)}\left(\frac{t}{1+\lambda t}\right)^{n-2}+ \\
& +\frac{1}{\lambda^{3}} \int \frac{t^{n-3}}{(1+\lambda t)^{n-2}} d t .
\end{aligned}
$$

Repetindo o método $n$ vezes, obtém-se:

$$
\int \frac{t^{n}}{(1+\lambda t)^{n+1}} d t=\frac{-1}{\lambda n}\left(\frac{t}{1+\lambda t}\right)^{n}-\frac{1}{\lambda^{2}(n-1)}\left(\frac{t}{1+\lambda t}\right)^{n-1}+\cdots+\frac{1}{\lambda^{n}} \int \frac{1}{(1+\lambda t)^{1}} d t
$$

ou melhor,

$$
\int \frac{t^{n}}{(1+\lambda t)^{n+1}} d t=\frac{-1}{\lambda n}\left(\frac{t}{1+\lambda t}\right)^{n}-\frac{1}{\lambda^{2}(n-1)}\left(\frac{t}{1+\lambda t}\right)^{n-1}+\cdots+\frac{1}{\lambda^{n}} \ln \left[\mathrm{e}_{\lambda}(t)\right],
$$


que pode ser resumida em

$$
\int \frac{t^{n}}{(1+\lambda t)^{n+1}} d t=\frac{1}{\lambda^{n}} \ln \left[\mathrm{e}_{\lambda}(t)\right]-\sum_{i=1}^{n} \frac{1}{\lambda^{i}(n-i+1)}\left(\frac{t}{1+\lambda t}\right)^{n-i+1} .
$$

Substituindo o resultado da Eq. (1.16) na Eq. (1.9) tem-se:

$$
\frac{d^{n}}{d \lambda^{n}} \ln \left[\mathrm{e}_{\lambda}(t)\right]=(-1)^{n} \cdot n !\left[\frac{1}{\lambda^{n}} \ln \left[\mathrm{e}_{\lambda}(t)\right]-\sum_{i=1}^{n} \frac{1}{\lambda^{i}(n-i+1)}\left(\frac{t}{1+\lambda t}\right)^{n-i+1}\right]
$$

Desta forma, obtém-se a $n$-ésima derivada de $\ln \left[\mathrm{e}_{\lambda}(t)\right]$. Utilizando o fato de que

$$
\frac{d}{d \lambda} \ln \left[\mathrm{e}_{\lambda}(t)\right]=\frac{1}{\mathrm{e}_{\lambda}(t)} \frac{d}{d \lambda} \mathrm{e}_{\lambda}(t)
$$

pode-se escrever:

$$
\frac{d^{n}}{d \lambda^{n}} \ln \left[\mathrm{e}_{\lambda}(t)\right]=\frac{d^{n-1}}{d \lambda^{n-1}} \frac{d}{d \lambda} \ln \left[\mathrm{e}_{\lambda}(t)\right]=\frac{d^{n-1}}{d \lambda^{n-1}}\left[\frac{1}{\mathrm{e}_{\lambda}(t)} \frac{d}{d \lambda} \mathrm{e}_{\lambda}(t)\right] .
$$

Ao continuar esse processo e utilizar a regra do produto, verifica-se que

$$
\begin{aligned}
& \frac{d^{n}}{d \lambda^{n}} \ln \left[\mathrm{e}_{\lambda}(t)\right]=\frac{d^{n-2}}{d \lambda^{n-2}} \frac{d}{d \lambda}\left[\frac{1}{\mathrm{e}_{\lambda}(t)} \frac{d}{d \lambda} \mathrm{e}_{\lambda}(t)\right]=\frac{d^{n-2}}{\lambda^{n-2}}\left[\frac{d}{d \lambda} \frac{1}{\mathrm{e}_{\lambda}(t)} \frac{d}{d \lambda} \mathrm{e}_{\lambda}(t)+\frac{1}{\mathrm{e}_{\lambda}(t)} \frac{d^{2}}{d \lambda^{2}} \mathrm{e}_{\lambda}(t)\right]= \\
& \frac{d^{n-3}}{d \lambda^{n-3}}\left[\frac{d^{2}}{d \lambda^{2}} \frac{1}{\mathrm{e}_{\lambda}(t)} \frac{d}{d \lambda} \mathrm{e}_{\lambda}(t)+2 \frac{d}{d \lambda} \frac{1}{\mathrm{e}_{\lambda}(t)} \frac{d^{2}}{d \lambda^{2}} \mathrm{e}_{\lambda}(t)+\frac{1}{\mathrm{e}_{\lambda}(t)} \frac{d^{3}}{d \lambda^{3}} \mathrm{e}_{\lambda}(t)\right]= \\
& \frac{d^{n-4}}{d \lambda^{n-4}}\left[\frac{d^{3}}{d \lambda^{3}} \frac{1}{\mathrm{e}_{\lambda}(t)} \frac{d}{d \lambda} \mathrm{e}_{\lambda}(t)+3 \frac{d^{2}}{d \lambda^{2}} \frac{1}{\mathrm{e}_{\lambda}(t)} \frac{d^{2}}{d \lambda^{2}} \mathrm{e}_{\lambda}(t)+3 \frac{d}{d \lambda} \frac{1}{\mathrm{e}_{\lambda}(t)} \frac{d^{3}}{d \lambda^{3}} \mathrm{e}_{\lambda}(t)+\frac{1}{\mathrm{e}_{\lambda}(t)} \frac{d^{4}}{d \lambda^{4}} \mathrm{e}_{\lambda}(t)\right]= \\
& \vdots \\
& =\sum_{i=1}^{n}\left(\begin{array}{c}
n-1 \\
i-1
\end{array}\right) \frac{d^{(n-i)}}{d \lambda^{(n-i)}} \frac{1}{\mathrm{e}_{\lambda}(t)} \frac{d^{(i)}}{d \lambda^{(i)}} \mathrm{e}_{\lambda}(t)+\frac{1}{\mathrm{e}_{\lambda}(t)} \frac{d^{(n)}}{d \lambda^{(n)}} \mathrm{e}_{\lambda}(t) .
\end{aligned}
$$

Substituindo a Eq. (1.20) na Eq. (1.17) obtém-se

$$
\begin{aligned}
\sum_{i=1}^{n}\left(\begin{array}{c}
n-1 \\
i-1
\end{array}\right) & \frac{d^{(n-i)}}{d \lambda^{(n-i)}} \frac{1}{\mathrm{e}_{\lambda}(t)} \frac{d^{(i)}}{d \lambda^{(i)}} \mathrm{e}_{\lambda}(t)+\frac{1}{\mathrm{e}_{\lambda}(t)} \frac{d^{(n)}}{d \lambda^{(n)}} \mathrm{e}_{\lambda}(t)= \\
& =(-1)^{n} \cdot n !\left[\frac{1}{\lambda^{n}} \ln \left[\mathrm{e}_{\lambda}(t)\right]-\sum_{j=1}^{n} \frac{1}{\lambda^{j}(n-i+1)}\left(\frac{t}{1+\lambda t}\right)^{n-j+1}\right],
\end{aligned}
$$

que, com os devidos ajustes, pode ser escrito como

$$
\begin{aligned}
\frac{d^{n}}{d \lambda^{n}} \mathrm{e}_{\lambda}(t) & =\mathrm{e}_{\lambda}(t)\left\{(-1)^{n} n !\left[\frac{\ln \left[\mathrm{e}_{\lambda}(t)\right]}{\lambda^{n}}-\sum_{j=1}^{n} \frac{\lambda^{-j}}{(n+1-j)}\left(\frac{t}{1+\lambda t}\right)^{n+1-j}\right]+\right. \\
& \left.-\sum_{i=1}^{n-1}\left(\begin{array}{c}
n-1 \\
i-1
\end{array}\right)\left(\frac{d^{(n-i)}}{d \lambda^{(n-i)}}\left[\mathrm{e}_{\lambda}(t)\right]^{-1}\right)\left(\frac{d^{(i)}}{d \lambda^{(i)}} \mathrm{e}_{\lambda}(t)\right)\right\}
\end{aligned}
$$


Vale lembrar que nas equações acima a expressão $\frac{d^{(k)}}{d \lambda^{(k)}} f_{\lambda}(t)$ faz referência a $k$-ésima derivada da função $f_{\lambda}(t)$ em relação a $\lambda, k$ vezes.

Uma próxima etapa de trabalho é verificar se existe uma expressão que simplifica o somatório de derivadas que aparece no lado direito da Eq. (1.22). 


\section{Apêndice}

\section{Generalização das demais funções Trigonométricas e Hiperbólicas}

Ao longo do texto, foram apresentadas as principais funções trigonométricas e hiperbólicas generalizadas, bem como suas propriedades e características. Indicouse lá que, mediante manipulações algébricas relativamente simples, era possível obter as demais generalizações. Reserva-se este espaço para apresentar, como curiosidade, estas outras funções, feita sem qualquer pretensão ou discussão mais aprofundada. Inicia-se esta apresentação pelas funções trigonométricas.

\subsection{Funções trigonométricas generalizadas}

No Cap. 5 foram apresentadas novas definições para generalizações das funções trigonométricas, cosseno $c_{\lambda}(\omega, t)$ e seno $s_{\lambda}(\omega, t)$. Tais definições se baseiam em pressupostos da nova generalização da função exponencial, que proporciona a seguinte relação:

$$
\mathrm{e}^{i \omega t} \rightarrow\left[\mathrm{e}_{\lambda}(t)\right]^{i \omega}
$$

Utilizando as definições de Euler e a relação da Eq. (2.23), obtém-se:

$$
\begin{aligned}
& \mathrm{c}_{\lambda}(\omega, t)=\frac{\left[\mathrm{e}_{\lambda}(t)\right]^{i \omega}+\left[\mathrm{e}_{\lambda}(t)\right]^{-i \omega}}{2}=\cos \left\{\omega \ln \left[\mathrm{e}_{\lambda}(t)\right]\right\} \\
& \mathrm{s}_{\lambda}(\omega, t)=\frac{\left[\mathrm{e}_{\lambda}(t)\right]^{i \omega}-\left[\mathrm{e}_{\lambda}(t)\right]^{-i \omega}}{2 i}=\operatorname{sen}\left\{\omega \ln \left[\mathrm{e}_{\lambda}(t)\right]\right\} .
\end{aligned}
$$

Na trigonometria tradicional, as funções tangente, cotangente, secante e cossecante podem ser obtidas mediante simples operações com as funções cosseno e 
seno, à saber:

$$
\begin{aligned}
& \tan (\omega t)=\frac{\operatorname{sen}(\omega t)}{\cos (\omega t)}=\frac{\mathrm{e}^{i \omega t}-\mathrm{e}^{-i \omega t}}{i\left(\mathrm{e}^{i \omega t}+\mathrm{e}^{-i \omega t}\right)} \\
& \cot (\omega t)=\frac{\cos (\omega t)}{\operatorname{sen}(\omega t)}=\frac{i\left(\mathrm{e}^{i \omega t}+\mathrm{e}^{-i \omega t}\right)}{\mathrm{e}^{i \omega t}-\mathrm{e}^{-i \omega t}} ; \\
& \sec (\omega t)=\frac{1}{\cos (\omega t)}=\frac{2}{\mathrm{e}^{i \omega t}+\mathrm{e}^{-i \omega t}} ; \\
& \csc (\omega t)=\frac{1}{\operatorname{sen}(\omega t)}=\frac{2 i}{\mathrm{e}^{i \omega t}-\mathrm{e}^{-i \omega t}}
\end{aligned}
$$

A forma generalizada é obtida mediante a relação da Eq. (2.23), em cada uma das definições originais.

\subsubsection{Função tangente generalizada: $t_{\lambda}(\omega, t)$}

Utilizando a Eq. (2.25a) com a relação da Eq. (2.23), obtém-se a generalização da função tangente:

$$
\mathrm{t}_{\lambda}(\omega, t)=\frac{\mathrm{s}_{\lambda}(\omega, t)}{\mathrm{c}_{\lambda}(\omega, t)}=\frac{\left[\mathrm{e}_{\lambda}(t)\right]^{i \omega}-\left[\mathrm{e}_{\lambda}(t)\right]^{-i \omega}}{i\left\{\left[\mathrm{e}_{\lambda}(t)\right]^{i \omega}+\left[\mathrm{e}_{\lambda}(t)\right]^{-i \omega}\right\}}
$$

O comportamento desta família de funções podem ser observados com maiores detalhes na Fig. 2.1a. Nela são plotadas as curvas para os valores de $\lambda$ iguais a 1, 2 e 3, respectivamente. É possível ainda, observar que todas as curvas possuem formato que acompanham a função tangente original. Contudo, elas vão "espichando" de modo mais acelerado à medida que $\lambda$ aumenta.

\subsubsection{Função cotangente generalizada: $\cot _{\lambda}(\omega, t)$}

Para obter a generalização da função cotangente, basta utilizar a definição apresentada na Eq. (2.25b) e a relação da Eq. (2.23). Desta forma,

$$
\cot _{\lambda}(\omega, t)=\frac{\mathrm{c}_{\lambda}(\omega, t)}{\mathrm{s}_{\lambda}(\omega, t)}=\frac{i\left\{\left[\mathrm{e}_{\lambda}(t)\right]^{i \omega}+\left[\mathrm{e}_{\lambda}(t)\right]^{-i \omega}\right\}}{\left[\mathrm{e}_{\lambda}(t)\right]^{i \omega}-\left[\mathrm{e}_{\lambda}(t)\right]^{-i \omega}} .
$$

Outra forma de obter esta generalização é calcular $\cot _{\lambda}(\omega, t)=1 / t_{\lambda}(\omega, t)$. Algumas curvas desta família de funções podem ser observadas na Fig. 2.1b. Nela plota-se as curvas para $\lambda=1, \lambda=2$ e $\lambda=3$, além de $\omega=5$. Percebe-se, nestas curvas, comportamento semelhante aos da função original. 

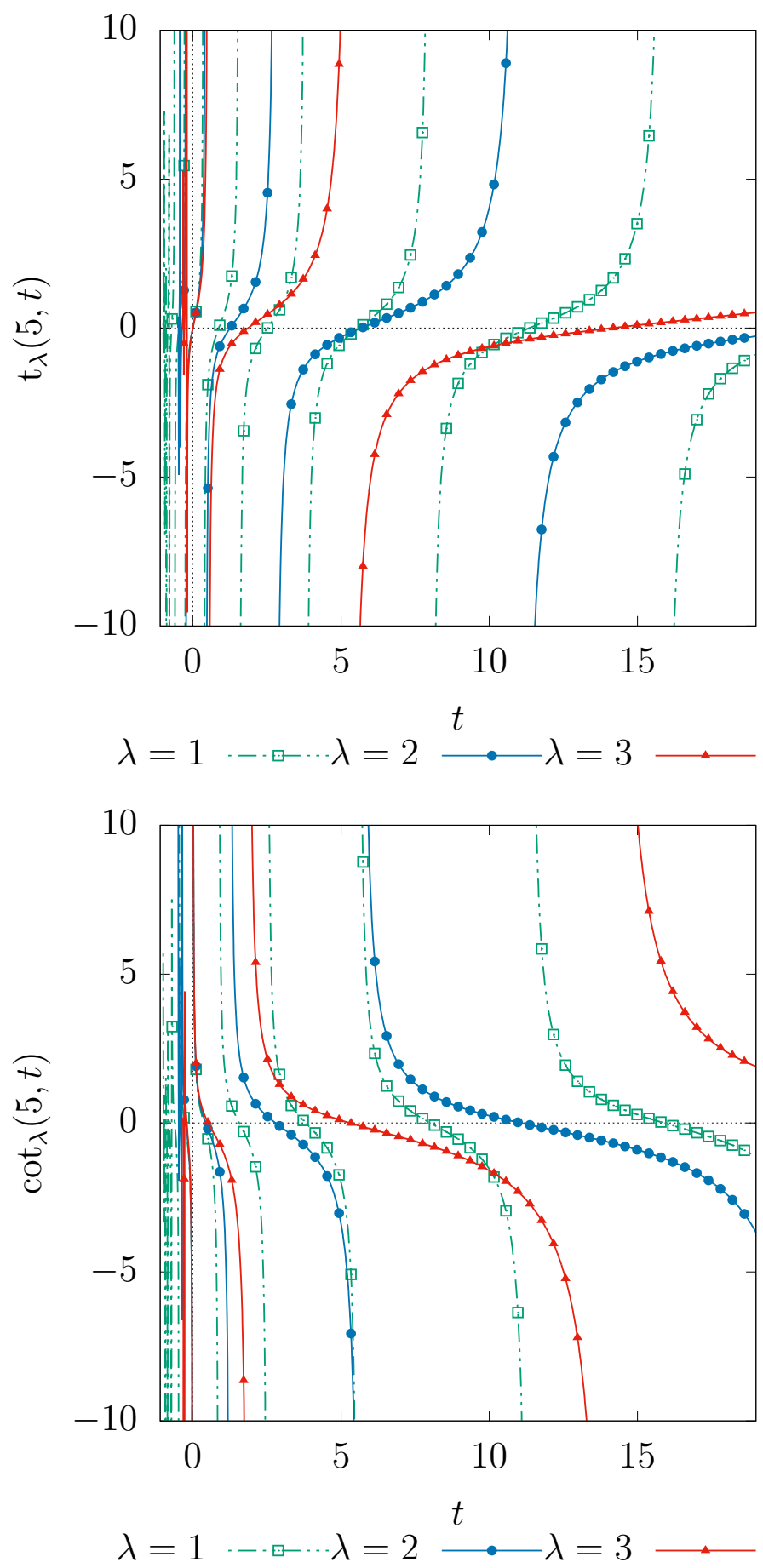

Figura 2.1: (a) Função tangente generalizada $t_{\lambda}(5, t)$ para $\lambda=1, \lambda=2$ e $\lambda=3$. Curvas possuem características semelhantes à tangente original. (b) Função cotangente generalizada $\cot _{\lambda}(5, t)$ para valores de $\lambda=1, \lambda=2$ e $\lambda=3$. As curvas se assemelham às obtidas pela função original. 


\subsubsection{Função secante generalizada: $\operatorname{sc}_{\lambda}(\omega, t)$}

A generalização da função secante pode ser escrita como

$$
\mathrm{sc}_{\lambda}(\omega, t)=\frac{1}{\mathrm{c}_{\lambda}(\omega, t)}=\frac{2}{\left[\mathrm{e}_{\lambda}(t)\right]^{i \omega}+\left[\mathrm{e}_{\lambda}(t)\right]^{-i \omega}} .
$$

Essa expressão pode ser obtida à partir da Eq. (2.25c) em conjunto com a relação da Eq. (2.23). O comportamento das curvas desta família de funções é exemplificado na Fig. 2.2. Nela, estão representadas as curvas para valores de $\lambda$ iguais a 1, 2 e 3, respectivamente. Percebe-se as mesmas características da função original, com uma variação nos períodos.

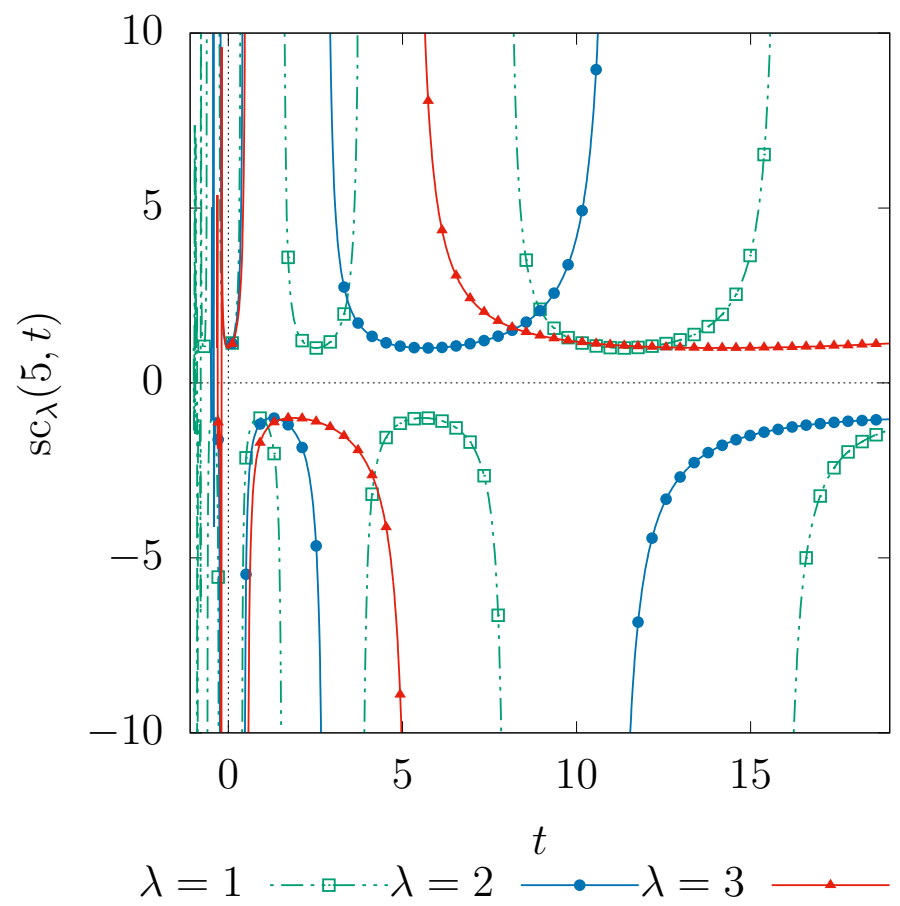

Figura 2.2: Função secante generalizada $\mathrm{s}_{\lambda}(5, t)$ para valores de $\lambda=1, \lambda=2$ e $\lambda=3$. Os períodos vão aumentando à medida que $\lambda$ aumenta.

\subsubsection{Função cossecante generalizada: $\csc _{\lambda}(\omega, t)$}

A Fig. 2.3 apresenta o comportamento de algumas curvas da função cossecante generalizada. A definição desta generalização se dá à partir da função cossecante original, apresentada na Eq. (2.25d), combinada com a relação da Eq. (2.23), o que gera a seguinte expressão:

$$
\csc _{\lambda}(\omega, t)=\frac{1}{\mathrm{~s}_{\lambda}(\omega, t)}=\frac{2 i}{\left[\mathrm{e}_{\lambda}(t)\right]^{i \omega}-\left[\mathrm{e}_{\lambda}(t)\right]^{-i \omega}} .
$$




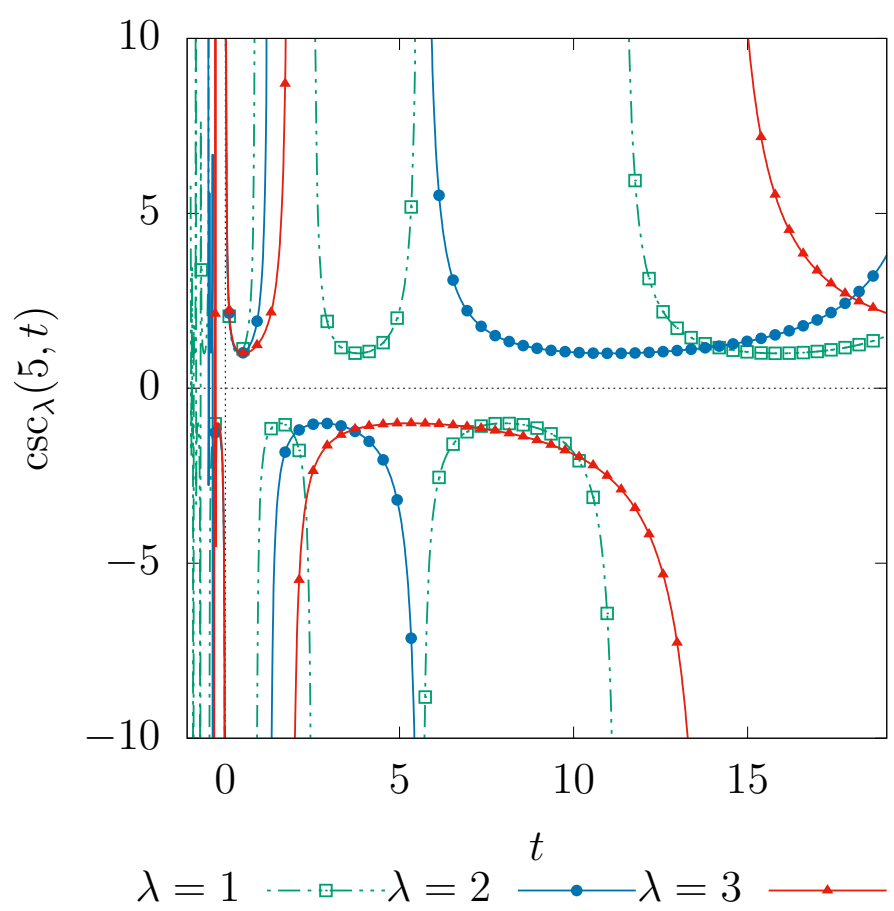

Figura 2.3: Função cossecante generalizada $\csc _{\lambda}(5, t)$, para valores de $\lambda=1, \lambda=2$ e $\lambda=3$.

\subsection{Funções hiperbólicas generalizadas}

As funções hiperbólicas também podem ser escritas através da fórmula de Euler. Ao utilizar a relação apresentada na Eq. (2.23), obtemos as funções hiperbólicas generalizadas, de acordo com discussão apresentada no Cap. 5. Lá definiu-se as generalizações para as funções cosseno e seno hiperbólicos de tal forma que:

$$
\begin{aligned}
\operatorname{ch}_{\lambda}(\omega, t) & =\frac{\left[\mathrm{e}_{\lambda}(t)\right]^{\omega}+\left[\mathrm{e}_{\lambda}(t)\right]^{-\omega}}{2} \\
\operatorname{sh}_{\lambda}(\omega, t) & =\frac{\left[\mathrm{e}_{\lambda}(t)\right]^{\omega}-\left[\mathrm{e}_{\lambda}(t)\right]^{-\omega}}{2} .
\end{aligned}
$$

No texto deste Cap. 5 foi indicado que as demais generalizações das funções hiperbólicas, poderiam ser obtidas mediante operações simples com essas duas funções principais. Segue, como curiosidade, as demais definições. 
2.2.1 Função tangente hiperbólica generalizada: $\operatorname{th}_{\lambda}(\omega, t)$

A função tangente hiperbólica também pode ser escrita utilizando a função exponencial. Desta forma,

$$
\tanh (\omega t)=\frac{\operatorname{senh}(\omega t)}{\cosh (\omega t)}=\frac{\mathrm{e}^{\omega t}-\mathrm{e}^{-\omega t}}{\mathrm{e}^{\omega t}+\mathrm{e}^{-\omega t}}
$$

Utilizando a relação da Eq. (2.23) na definição original, Eq. (2.31), obtém-se a seguinte generalização

$$
\operatorname{th}_{\lambda}(\omega, t)=\frac{\operatorname{sh}_{\lambda}(\omega, t)}{\operatorname{ch}_{\lambda}(\omega, t)}=\frac{\left[\mathrm{e}_{\lambda}(t)\right]^{\omega}-\left[\mathrm{e}_{\lambda}(t)\right]^{-\omega}}{\left[\mathrm{e}_{\lambda}(t)\right]^{\omega}+\left[\mathrm{e}_{\lambda}(t)\right]^{-\omega}}
$$

O comportamento das curvas desta família de funções são exemplificados na Fig. 2.4. Nela são exibidas as curvas para $\lambda=-1, \lambda=2$ e $\lambda=3$. Percebe-se disposição que acompanha tudo que é próprio da função original.

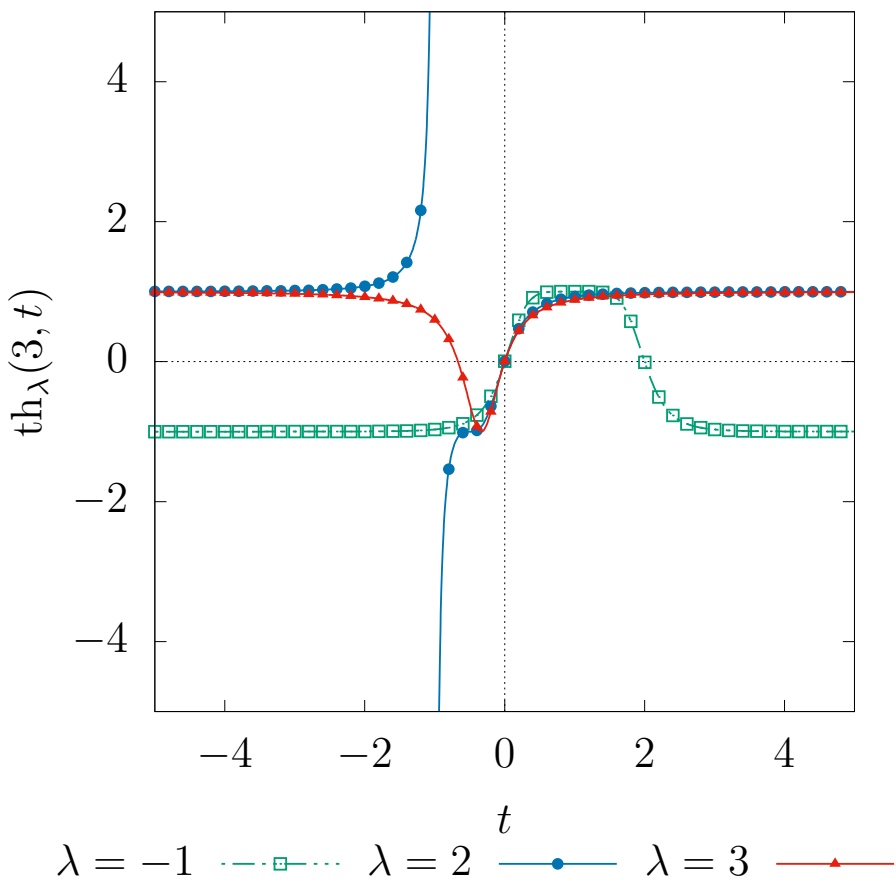

Figura 2.4: Função tangente hiperbólica generalizada $\operatorname{th}_{\lambda}(3, t)$, para valores de $\lambda=-1, \lambda=2$ e $\lambda=3$. 


\subsubsection{Função cotangente hiperbólica generalizada: $\operatorname{cth}_{\lambda}(\omega, t)$}

A definição da função cotangente hiperbólica tradicional, pode ser obtido pela igualdade $\operatorname{coth}(\omega t)=1 / \tanh (\omega t)$. Desta forma,

$$
\operatorname{coth}(\omega t)=\frac{\cosh (\omega t)}{\operatorname{senh}(\omega t)}=\frac{\mathrm{e}^{\omega t}+\mathrm{e}^{-\omega t}}{\mathrm{e}^{\omega t}-\mathrm{e}^{-\omega t}}
$$

Utilizando a relação da Eq. (2.23) aplicada na definição da Eq. (2.33), chega-se a uma generalização para a função cotangente hiperbólica:

$$
\operatorname{cth}_{\lambda}(\omega, t)=\frac{\operatorname{ch}_{\lambda}(\omega, t)}{\operatorname{sh}_{\lambda}(\omega, t)}=\frac{\left[\mathrm{e}_{\lambda}(t)\right]^{\omega}+\left[\mathrm{e}_{\lambda}(t)\right]^{-\omega}}{\left[\mathrm{e}_{\lambda}(t)\right]^{\omega}-\left[\mathrm{e}_{\lambda}(t)\right]^{-\omega}} .
$$

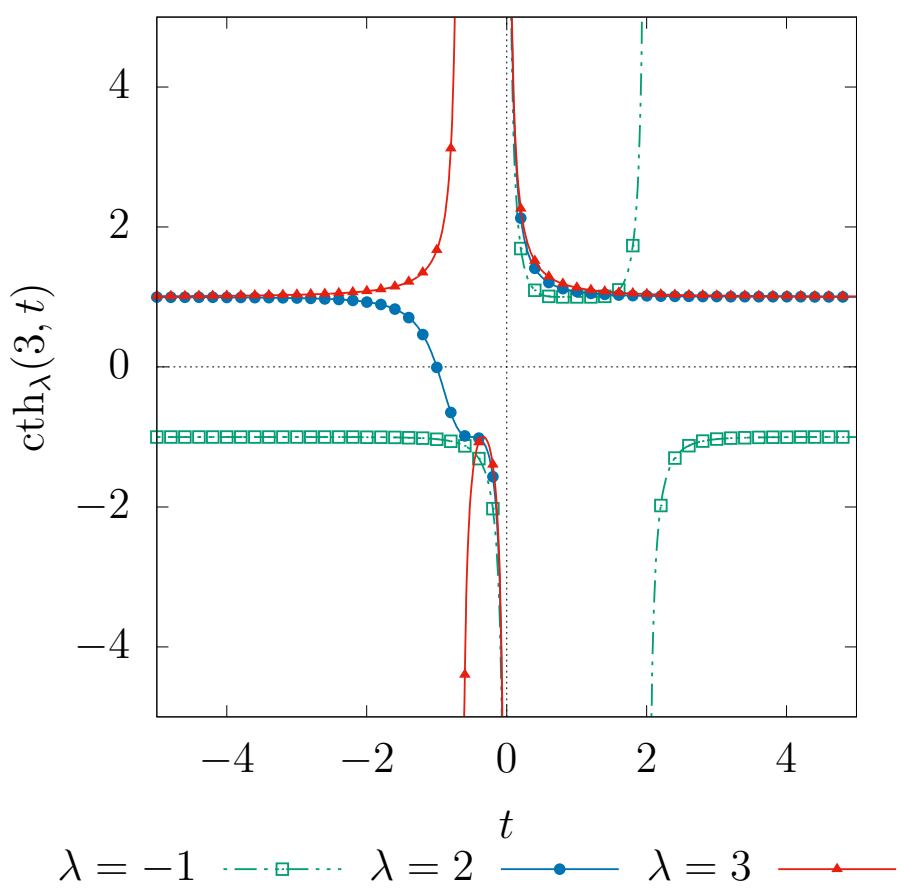

Figura 2.5: Função cotangente hiperbólica generalizada $\operatorname{cth}_{\lambda}(3, t)$, para valores de $\lambda=-1, \lambda=2$ e $\lambda=3$.

A Fig. 2.5 apresenta o comportamento desta família de funções para os valores de $\lambda=-1, \lambda=2$ e $\lambda=3$, além de $\omega=3$.

\subsubsection{Função secante hiperbólica generalizada: $\operatorname{sch}_{\lambda}(\omega, t)$}

$\mathrm{Na}$ análise tradicional, a função secante hiperbólica pode ser definida como o inverso multiplicativo da função cosseno hiperbólico. Dessa forma,

$$
\operatorname{sech}(\omega t)=\frac{1}{\cosh (\omega t)}=\frac{2}{\mathrm{e}^{\omega t}+\mathrm{e}^{-\omega t}}
$$


Seguindo os mesmos critérios das generalizações anteriores e utilizando a relação apresentada na Eq. 2.23, generaliza-se a função secante hiperbólica da seguinte forma:

$$
\operatorname{sch}_{\lambda}(\omega, t)=\frac{1}{\operatorname{ch}_{\lambda}(\omega, t)}=\frac{2}{\left[\mathrm{e}_{\lambda}(t)\right]^{\omega}+\left[\mathrm{e}_{\lambda}(t)\right]^{-\omega}} .
$$

O comportamento desta família de funções, para valores de $\lambda=-1, \lambda=2 \mathrm{e}$ $\lambda=3$, pode ser observado na Fig. 2.6.

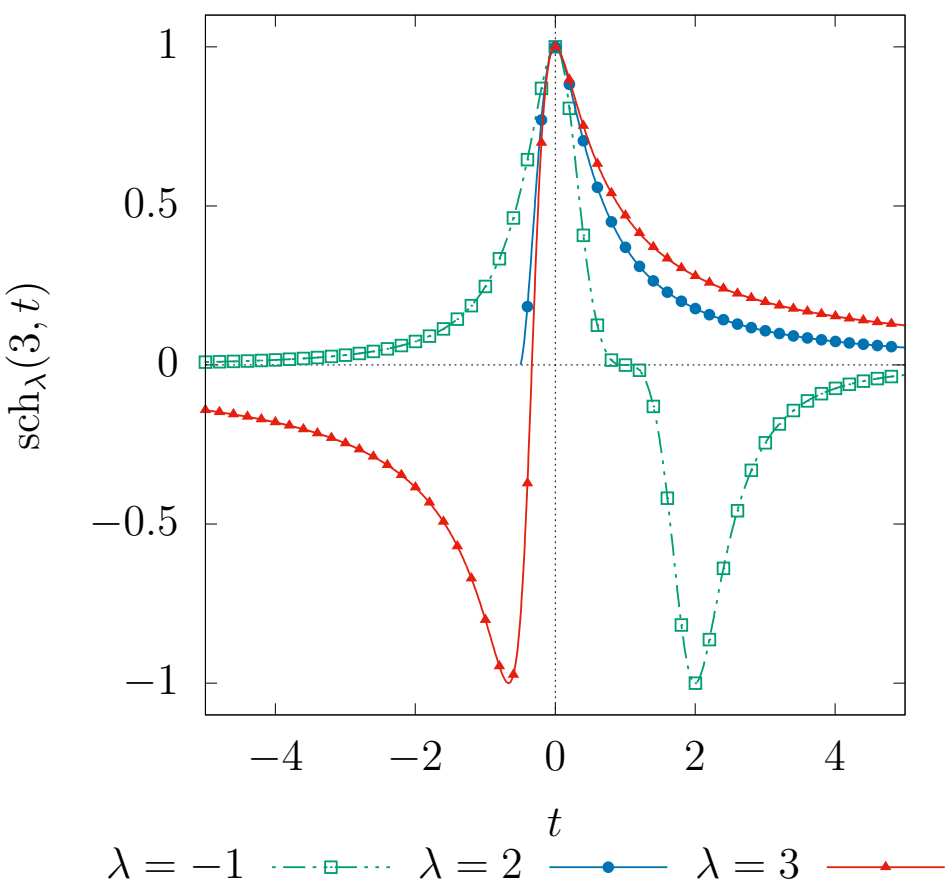

Figura 2.6: Função secante hiperbólica generalizada $\operatorname{sch}_{\lambda}(3, t)$, para valores de $\lambda=-1, \lambda=2$ e $\lambda=3$.

\subsubsection{Função cossecante hiperbólica generalizada: $\operatorname{csch}_{\lambda}(\omega, t)$}

A função cossecante hiperbólica pode ser obtida pelo inverso multiplicativo da função seno hiperbólico, desta forma:

$$
\operatorname{cosech}(\omega t)=\frac{1}{\operatorname{senh}(\omega t)}=\frac{2}{\mathrm{e}^{\omega t}-\mathrm{e}^{-\omega t}}
$$

Utilizando a relação exposta na Eq. (2.23), na definição da Eq. (2.37), obtémse a função cossecante hiperbólica generalizada,

$$
\operatorname{csch}_{\lambda}(\omega, t)=\frac{1}{\operatorname{sh}_{\lambda}(\omega, t)}=\frac{2}{\left[\mathrm{e}_{\lambda}(t)\right]^{\omega}-\left[\mathrm{e}_{\lambda}(t)\right]^{-\omega}} .
$$


O comportamento desta família de funções pode ser observado na Fig. . Nela são representadas as curvas para os valores de $\lambda=-1, \lambda=2$ e $\lambda=3$.

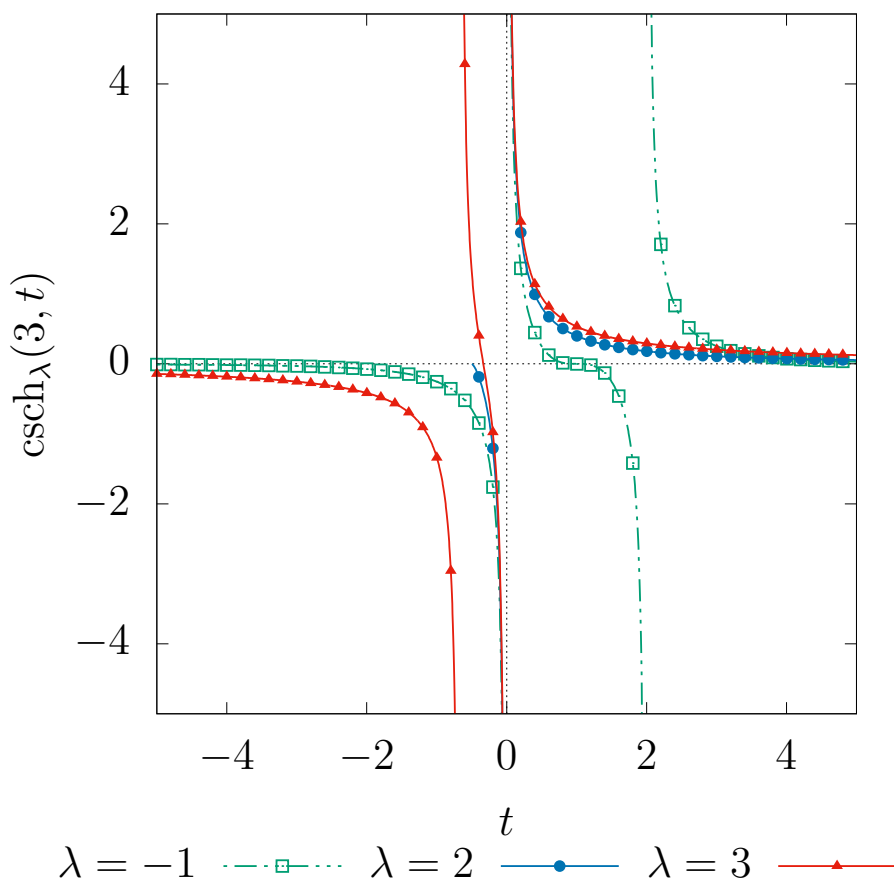

Figura 2.7: Função cossecante hiperbólica generalizada $\operatorname{csch}_{\lambda}(3, t)$, para valores de $\lambda=-1, \lambda=2$ e $\lambda=3$.

Em trabalhos futuros pretende-se estudar melhor estas generalizações, bem como suas possíveis aplicações. 


\section{Apêndice}

\section{Algumas demontrações}

No Cap. 5 apresentamos uma série de propriedades relativas às novas generalizações das funções trigonométricas e hiperbólicas. Reserva-se este espaço às demonstrações de algumas dessas propriedades.

\subsection{Relação fundamental da trigonometria}

A Eq. (5.51), do Cap. 5, traz a seguinte relação:

$$
\mathrm{c}_{\lambda}^{2}(\omega, t)+\mathrm{s}_{\lambda}^{2}(\omega, t)=1, \forall \omega, \lambda, t
$$

Esse resultado pode ser verificado, expandindo-se os quadrados das definições de cada função envolvida. De fato,

$$
\begin{aligned}
& \mathrm{c}_{\lambda}^{2}(\omega, t)+\mathrm{s}_{\lambda}^{2}(\omega, t)=\left\{\frac{\left[\mathrm{e}_{\lambda}(t)\right]^{i \omega}+\left[\mathrm{e}_{\lambda}(t)\right]^{-i \omega}}{2}\right\}^{2}+\left\{\frac{\left[\mathrm{e}_{\lambda}(t)\right]^{i \omega}-\left[\mathrm{e}_{\lambda}(t)\right]^{-i \omega}}{2 i}\right\}^{2} \\
& =\frac{1}{4}\left\{\left[\mathrm{e}_{\lambda}(t)\right]^{2 i \omega}+2 \frac{\left[\mathrm{e}_{\lambda}(t)\right]^{i \omega}}{\left[\mathrm{e}_{\lambda}(t)\right]^{i \omega}}+\left[\mathrm{e}_{\lambda}(t)\right]^{-2 i \omega}-\left[\mathrm{e}_{\lambda}(t)\right]^{2 i \omega}+2 \frac{\left[\mathrm{e}_{\lambda}(t)\right]^{i \omega}}{\left[\mathrm{e}_{\lambda}(t)\right]^{i \omega}}-\left[\mathrm{e}_{\lambda}(t)\right]^{-2 i \omega}\right\} \\
& =\frac{1}{4}\{2+2\}=\frac{4}{4}=1
\end{aligned}
$$

para quaisquer valores de $\lambda, \omega, t$. Esse resultado é muito consistente quando comparado com a relação original; lá, partido do teorema de Pitágoras, chega-se à relação unitária, mediante uma divisão. Assim, é esperado que, em uma generalização, este resultado seja mantido. 


\subsection{Relação de Euler}

A Eq. (5.52) apresenta a generalização da fórmula de Euler. Considerando que $f(t)=\mathrm{e}^{\ln [f(t)]}$, se $f$ for definida, tem-se que:

$$
\begin{aligned}
{\left[\mathrm{e}_{\lambda}(t)\right]^{ \pm i \omega} } & =\mathrm{e}^{\ln \left[\mathrm{e}_{\lambda}(t)\right]^{ \pm i \omega}}=\mathrm{e}^{ \pm i \omega \ln \left[\mathrm{e}_{\lambda}(t)\right]} \\
& =\cos \left\{\omega \ln \left[\mathrm{e}_{\lambda}(t)\right]\right\} \pm i \operatorname{sen}\left\{\omega \ln \left[\mathrm{e}_{\lambda}(t)\right]\right\} \\
& =\mathrm{c}_{\lambda}(\omega, t) \pm i \mathrm{~s}_{\lambda}(\omega, t),
\end{aligned}
$$

o que demonstra nosso resultado.

\subsection{Produtos entre funções e somas de $\operatorname{arcos}$}

O Cap. 5 apresenta uma série de propriedades baseadas em produtos de funções ou soma de argumentos das mesmas. Esta seção é dedicada a demonstração de algumas dessas propriedades, começando pelos produtos.

\subsubsection{Produtos}

No decorrer do texto, foram indicadas algumas propriedades que envolviam produtos de funções trigonométricas generalizadas. Essas propriedades possuem certa ligação com propriedades das funções usuais. Como a generalização proposta apresenta duas variáveis, existem duas possibilidades de produtos. Inicialmente, verificam-se os resultados em que, dada uma frequência comum $\omega$, têm-se as variáveis principais, de cada função, diferentes.

a-) Produto de cossenos

$$
\begin{aligned}
\mathrm{c}_{\lambda}(\omega, a) \mathrm{c}_{\lambda}(\omega, b)=\left\{\frac{\left[\mathrm{e}_{\lambda}(a)\right]^{i \omega}+\left[\mathrm{e}_{\lambda}(a)\right]^{-i \omega}}{2}\right\}\left\{\frac{\left.\mathrm{e}_{\lambda}(b)\right]^{i \omega}+\left[\mathrm{e}_{\lambda}(b)\right]^{-i \omega}}{2}\right\} \\
=\frac{1}{4}\left\{\left[\mathrm{e}_{\lambda}(a) \mathrm{e}_{\lambda}(b)\right]^{i \omega}+\left[\frac{\mathrm{e}_{\lambda}(a)}{\mathrm{e}_{\lambda}(b)}\right]^{i \omega}+\left[\frac{\mathrm{e}_{\lambda}(a)}{\mathrm{e}_{\lambda}(b)}\right]^{-i \omega}+\left[\mathrm{e}_{\lambda}(a) \mathrm{e}_{\lambda}(b)\right]^{-i \omega}\right\} \\
=\frac{1}{4}\left\{\left[\mathrm{e}_{\lambda}\left(a \oplus_{\lambda} b\right)\right]^{i \omega}+\left[\mathrm{e}_{\lambda}\left(a \ominus_{\lambda} b\right)\right]^{i \omega}+\left[\mathrm{e}_{\lambda}\left(a \ominus_{\lambda} b\right)\right]^{-i \omega}+\left[\mathrm{e}_{\lambda}\left(a \oplus_{\lambda} b\right)\right]^{-i \omega}\right\} \\
=\frac{1}{2}\left\{\frac{\left[\mathrm{e}_{\lambda}\left(a \oplus_{\lambda} b\right)\right]^{i \omega}+\left[\mathrm{e}_{\lambda}\left(a \oplus_{\lambda} b\right)\right]^{-i \omega}+\left[\mathrm{e}_{\lambda}\left(a \ominus_{\lambda} b\right)\right]^{i \omega}+\left[\mathrm{e}_{\lambda}\left(a \ominus_{\lambda} b\right)\right]^{-i \omega}}{2}\right\} \\
=\frac{1}{2}\left[\mathrm{c}_{\lambda}\left(\omega, a \oplus_{\lambda} b\right)+\mathrm{c}_{\lambda}\left(\omega, a \ominus_{\lambda} b\right)\right] .
\end{aligned}
$$


b-) Produto de senos

$$
\begin{aligned}
\mathrm{s}_{\lambda}(\omega, a) & \mathrm{s}_{\lambda}(\omega, b)=\left\{\frac{\left[\mathrm{e}_{\lambda}(a)\right]^{i \omega}-\left[\mathrm{e}_{\lambda}(a)\right]^{-i \omega}}{2 i}\right\}\left\{\frac{\left[\mathrm{e}_{\lambda}(b)\right]^{i \omega}-\left[\mathrm{e}_{\lambda}(b)\right]^{-i \omega}}{2 i}\right\} \\
& =-\frac{1}{4}\left\{\left[\mathrm{e}_{\lambda}(a) \mathrm{e}_{\lambda}(b)\right]^{i \omega}-\left[\frac{\mathrm{e}_{\lambda}(a)}{\mathrm{e}_{\lambda}(b)}\right]^{i \omega}-\left[\frac{\mathrm{e}_{\lambda}(a)}{\mathrm{e}_{\lambda}(b)}\right]^{-i \omega}+\left[\mathrm{e}_{\lambda}(a) \mathrm{e}_{\lambda}(b)\right]^{-i \omega}\right\} \\
& =-\frac{1}{4}\left\{\left[\mathrm{e}_{\lambda}\left(a \oplus_{\lambda} b\right)\right]^{i \omega}-\left[\mathrm{e}_{\lambda}\left(a \ominus_{\lambda} b\right)\right]^{i \omega}-\left[\mathrm{e}_{\lambda}\left(a \ominus_{\lambda} b\right)\right]^{-i \omega}+\left[\mathrm{e}_{\lambda}\left(a \oplus_{\lambda} b\right)\right]^{-i \omega}\right\} \\
& =-\frac{1}{2}\left\{\frac{\left[\mathrm{e}_{\lambda}\left(a \oplus_{\lambda} b\right)\right]^{i \omega}+\left[\mathrm{e}_{\lambda}\left(a \oplus_{\lambda} b\right)\right]^{-i \omega}-\left[\mathrm{e}_{\lambda}\left(a \ominus_{\lambda} b\right)\right]^{i \omega}-\left[\mathrm{e}_{\lambda}\left(a \ominus_{\lambda} b\right)\right]^{-i \omega}}{2}\right\} \\
& =-\frac{1}{2}\left[\mathrm{c}_{\lambda}\left(\omega, a \oplus_{\lambda} b\right)-\mathrm{c}_{\lambda}\left(\omega, a \ominus_{\lambda} b\right)\right] .
\end{aligned}
$$

c-) Produto de senos por cossenos

$$
\begin{aligned}
\mathrm{s}_{\lambda}(\omega, a) & \mathrm{c}_{\lambda}(\omega, b)=\left\{\frac{\left[\mathrm{e}_{\lambda}(a)\right]^{i \omega}-\left[\mathrm{e}_{\lambda}(a)\right]^{-i \omega}}{2 i}\right\}\left\{\frac{\left[\mathrm{e}_{\lambda}(b)\right]^{i \omega}+\left[\mathrm{e}_{\lambda}(b)\right]^{-i \omega}}{2}\right\} \\
= & \frac{1}{4 i}\left\{\left[\mathrm{e}_{\lambda}(a) \mathrm{e}_{\lambda}(b)\right]^{i \omega}+\left[\frac{\mathrm{e}_{\lambda}(a)}{\mathrm{e}_{\lambda}(b)}\right]^{i \omega}-\left[\frac{\mathrm{e}_{\lambda}(a)}{\mathrm{e}_{\lambda}(b)}\right]^{-i \omega}-\left[\mathrm{e}_{\lambda}(a) \mathrm{e}_{\lambda}(b)\right]^{-i \omega}\right\} \\
= & \frac{1}{4 i}\left\{\left[\mathrm{e}_{\lambda}\left(a \oplus_{\lambda} b\right)\right]^{i \omega}+\left[\mathrm{e}_{\lambda}\left(a \ominus_{\lambda} b\right)\right]^{i \omega}-\left[\mathrm{e}_{\lambda}\left(a \ominus_{\lambda} b\right)\right]^{-i \omega}-\left[\mathrm{e}_{\lambda}\left(a \oplus_{\lambda} b\right)\right]^{-i \omega}\right\} \\
= & \frac{1}{2}\left\{\frac{\left[\mathrm{e}_{\lambda}\left(a \oplus_{\lambda} b\right)\right]^{i \omega}-\left[\mathrm{e}_{\lambda}\left(a \oplus_{\lambda} b\right)\right]^{-i \omega}+\left[\mathrm{e}_{\lambda}\left(a \ominus_{\lambda} b\right)\right]^{i \omega}-\left[\mathrm{e}_{\lambda}\left(a \ominus_{\lambda} b\right)\right]^{-i \omega}}{2 i}\right\} \\
= & \frac{1}{2}\left[\mathrm{~s}_{\lambda}\left(\omega, a \oplus_{\lambda} b\right)-\mathrm{s}_{\lambda}\left(\omega, a \ominus_{\lambda} b\right)\right] .
\end{aligned}
$$

Desta forma, validamos as igualdades apresentadas nas Eq. (5.58).

Existem ainda possibilidades de produtos entre as funções trigonométricas generalizadas com a mesma variável principal e com frequências diferentes. Desta forma,

a-) Produto de cossenos

$$
\begin{aligned}
\mathrm{c}_{\lambda}\left(\omega_{1}, t\right) & \mathrm{c}_{\lambda}\left(\omega_{2}, t\right)=\left\{\frac{\left[\mathrm{e}_{\lambda}(t)\right]^{i \omega_{1}}+\left[\mathrm{e}_{\lambda}(t)\right]^{-i \omega_{1}}}{2}\right\}\left\{\frac{\left[\mathrm{e}_{\lambda}(t)\right]^{i \omega_{2}}+\left[\mathrm{e}_{\lambda}(t)\right]^{-i \omega_{2}}}{2}\right\} \\
= & \frac{1}{4}\left\{\left[\mathrm{e}_{\lambda}(t)\right]^{i\left(\omega_{1}+\omega_{2}\right)}+\left[\mathrm{e}_{\lambda}(t)\right]^{i\left(\omega_{1}-\omega_{2}\right)}+\left[\mathrm{e}_{\lambda}(t)\right]^{-i\left(\omega_{1}-\omega_{2}\right)}+\left[\mathrm{e}_{\lambda}(t)\right]^{-i\left(\omega_{1}+\omega_{2}\right)}\right\} \\
= & \frac{1}{4}\left\{\frac{\left[\mathrm{e}_{\lambda}(t)\right]^{i\left(\omega_{1}+\omega_{2}\right)}+\left[\mathrm{e}_{\lambda}(t)\right]^{-i\left(\omega_{1}+\omega_{2}\right)}+\left[\mathrm{e}_{\lambda}(t)\right]^{i\left(\omega_{1}-\omega_{2}\right)}+\left[\mathrm{e}_{\lambda}(t)\right]^{-i\left(\omega_{1}-\omega_{2}\right)}}{2}\right\} \\
= & \frac{1}{2}\left[\mathrm{c}_{\lambda}\left(\omega_{1}+\omega_{2}, t\right)+\mathrm{c}_{\lambda}\left(\omega_{1}-\omega_{2}, t\right)\right] .
\end{aligned}
$$


b-) Produto de senos

$$
\begin{aligned}
\mathrm{s}_{\lambda}\left(\omega_{1}, t\right) & \mathrm{s}_{\lambda}\left(\omega_{2}, t\right)=\left\{\frac{\left[\mathrm{e}_{\lambda}(t)\right]^{i \omega_{1}}-\left[\mathrm{e}_{\lambda}(t)\right]^{-i \omega_{1}}}{2 i}\right\}\left\{\frac{\left[\mathrm{e}_{\lambda}(t)\right]^{i \omega_{2}}-\left[\mathrm{e}_{\lambda}(t)\right]^{-i \omega_{2}}}{2 i}\right\} \\
& =-\frac{1}{4}\left\{\left[\mathrm{e}_{\lambda}(t)\right]^{i\left(\omega_{1}+\omega_{2}\right)}-\left[\mathrm{e}_{\lambda}(t)\right]^{i\left(\omega_{1}-\omega_{2}\right)}-\left[\mathrm{e}_{\lambda}(t)\right]^{-i\left(\omega_{1}-\omega_{2}\right)}+\left[\mathrm{e}_{\lambda}(t)\right]^{-i\left(\omega_{1}+\omega_{2}\right)}\right\} \\
& =-\frac{1}{2}\left\{\frac{\left[\mathrm{e}_{\lambda}(t)\right]^{i\left(\omega_{1}+\omega_{2}\right)}+\left[\mathrm{e}_{\lambda}(t)\right]^{-i\left(\omega_{1}+\omega_{2}\right)}-\left[\mathrm{e}_{\lambda}(t)\right]^{i\left(\omega_{1}-\omega_{2}\right)}-\left[\mathrm{e}_{\lambda}(t)\right]^{-i\left(\omega_{1}-\omega_{2}\right)}}{2}\right\} \\
& =-\frac{1}{2}\left[\mathrm{c}_{\lambda}\left(\omega_{1}+\omega_{2}, t\right)-\mathrm{c}_{\lambda}\left(\omega_{1}-\omega_{2}, t\right)\right] .
\end{aligned}
$$

c-) Produto de senos por cossenos

$$
\begin{aligned}
\mathrm{s}_{\lambda}\left(\omega_{1}, t\right) & \mathrm{c}_{\lambda}\left(\omega_{2}, t\right)=\left\{\frac{\left[\mathrm{e}_{\lambda}(t)\right]^{i \omega_{1}}-\left[\mathrm{e}_{\lambda}(t)\right]^{-i \omega_{1}}}{2 i}\right\}\left\{\frac{\left[\mathrm{e}_{\lambda}(t)\right]^{i \omega_{2}}+\left[\mathrm{e}_{\lambda}(t)\right]^{-i \omega_{2}}}{2}\right\} \\
= & \frac{1}{4 i}\left\{\left[\mathrm{e}_{\lambda}(t)\right]^{i\left(\omega_{1}+\omega_{2}\right)}+\left[\mathrm{e}_{\lambda}(t)\right]^{i\left(\omega_{1}-\omega_{2}\right)}-\left[\mathrm{e}_{\lambda}(t)\right]^{-i\left(\omega_{1}-\omega_{2}\right)}-\left[\mathrm{e}_{\lambda}(t)\right]^{-i\left(\omega_{1}+\omega_{2}\right)}\right\} \\
= & \frac{1}{2}\left\{\frac{\left[\mathrm{e}_{\lambda}(t)\right]^{i\left(\omega_{1}+\omega_{2}\right)}-\left[\mathrm{e}_{\lambda}(t)\right]^{-i\left(\omega_{1}+\omega_{2}\right)}+\left[\mathrm{e}_{\lambda}(t)\right]^{i\left(\omega_{1}-\omega_{2}\right)}-\left[\mathrm{e}_{\lambda}(t)\right]^{-i\left(\omega_{1}-\omega_{2}\right)}}{2 i}\right\} \\
= & \frac{1}{2}\left[\mathrm{~s}_{\lambda}\left(\omega_{1}+\omega_{2}, t\right)-\mathrm{s}_{\lambda}\left(\omega_{1}-\omega_{2}, t\right)\right] .
\end{aligned}
$$

Nestes termos, confirmamos as igualdades apresentadas nas Eq. (5.59).

\subsubsection{Soma de arcos}

Ainda no Cap. 5, apresentamos, nas Eq. (5.54), as chamadas propriedades de soma (ou diferença) generalizada de arcos, dadas por

$$
\begin{aligned}
& \mathrm{c}_{\lambda}\left(\omega, a \oplus_{\lambda} b\right)=\mathrm{c}_{\lambda}(\omega, a) \mathrm{c}_{\lambda}(\omega, b)-\mathrm{s}_{\lambda}(\omega, a) \mathrm{s}_{\lambda}(\omega, b), \\
& \mathrm{c}_{\lambda}\left(\omega, a \ominus_{\lambda} b\right)=\mathrm{c}_{\lambda}(\omega, a) \mathrm{c}_{\lambda}(\omega, b)+\mathrm{s}_{\lambda}(\omega, a) \mathrm{s}_{\lambda}(\omega, b), \\
& \mathrm{s}_{\lambda}\left(\omega, a \oplus_{\lambda} b\right)=\mathrm{s}_{\lambda}(\omega, a) \mathrm{c}_{\lambda}(\omega, b)+\mathrm{s}_{\lambda}(\omega, b) \mathrm{c}_{\lambda}(\omega, a), \\
& \mathrm{s}_{\lambda}\left(\omega, a \ominus_{\lambda} b\right)=\mathrm{s}_{\lambda}(\omega, a) \mathrm{c}_{\lambda}(\omega, b)-\mathrm{s}_{\lambda}(\omega, b) \mathrm{c}_{\lambda}(\omega, a) .
\end{aligned}
$$

Tais igualdades estão relacionadas com as propriedades de somas de arcos da trigonometria tradicional, em que, por exemplo,

$$
\cos [\omega(a+b)]=\cos (\omega a) \cos (\omega b)-\operatorname{sen}(\omega a) \operatorname{sen}(\omega b) .
$$

Para verificar essas propriedades, na versão generalizada, faz-se uso dos produtos entre funções trigonométricas generalizadas, os quais foram apresentados, também como propriedades, nas Eq. (5.58) e demonstradas nas Eq. (3.42) até Eq. (3.44) deste 
Apêndice. De posse desses resultados, parte-se então para a verificação da validade das propriedades relativas à soma (ou diferença) generalizada. Para tanto, no intuito de manter-se correspondência com as propriedades tradicionais exemplificada na Eq. (3.49), aproveita-se os resultados obtidos nas Eq. (3.42) e Eq. (3.43) e assim,

$$
\begin{aligned}
\mathrm{c}_{\lambda}(\omega, a) \mathrm{c}_{\lambda}(\omega, b)-\mathrm{s}_{\lambda}(\omega, a) \mathrm{s}_{\lambda}(\omega, b) & =\frac{1}{2}\left[\mathrm{c}_{\lambda}\left(\omega, a \oplus_{\lambda} b\right)+\mathrm{c}_{\lambda}\left(\omega, a \ominus_{\lambda} b\right)\right] \\
& +\frac{1}{2}\left[\mathrm{c}_{\lambda}\left(\omega, a \oplus_{\lambda} b\right)-\mathrm{c}_{\lambda}\left(\omega, a \ominus_{\lambda} b\right)\right] \\
& =\frac{1}{2}\left[2 \mathrm{c}_{\lambda}\left(\omega, a \oplus_{\lambda} b\right)\right] \\
& =\mathrm{c}_{\lambda}\left(\omega, a \oplus_{\lambda} b\right),
\end{aligned}
$$

o que valida a propriedade apresentada na Eq. (3.48a). Fazendo a soma entre as expressões dos resultados obtidos nas Eq. (3.42) e Eq. (3.43), consegue-se a seguinte simplificação:

$$
\begin{aligned}
\mathrm{c}_{\lambda}(\omega, a) \mathrm{c}_{\lambda}(\omega, b)+\mathrm{s}_{\lambda}(\omega, a) \mathrm{s}_{\lambda}(\omega, b) & =\frac{1}{2}\left[\mathrm{c}_{\lambda}\left(\omega, a \oplus_{\lambda} b\right)+\mathrm{c}_{\lambda}\left(\omega, a \ominus_{\lambda} b\right)\right] \\
& -\frac{1}{2}\left[\mathrm{c}_{\lambda}\left(\omega, a \oplus_{\lambda} b\right)-\mathrm{c}_{\lambda}\left(\omega, a \ominus_{\lambda} b\right)\right] \\
& =\frac{1}{2}\left[2 \mathrm{c}_{\lambda}\left(\omega, a \ominus_{\lambda} b\right)\right] \\
& =\mathrm{c}_{\lambda}\left(\omega, a \ominus_{\lambda} b\right),
\end{aligned}
$$

o que valida a propriedade apresentada na Eq. (3.48b).

A Eq. (3.44) pode ser utilizada para o seguinte cálculo:

$$
\begin{aligned}
\mathrm{s}_{\lambda}(\omega, a) \mathrm{c}_{\lambda}(\omega, b) & +\mathrm{s}_{\lambda}(\omega, b) \mathrm{c}_{\lambda}(\omega, a)=\frac{1}{2}\left[\mathrm{~s}_{\lambda}\left(\omega, a \oplus_{\lambda} b\right)-\mathrm{s}_{\lambda}\left(\omega, a \ominus_{\lambda} b\right)\right] \\
& +\frac{1}{2}\left[\mathrm{~s}_{\lambda}\left(\omega, a \oplus_{\lambda} b\right)-\mathrm{s}_{\lambda}\left(\omega, b \ominus_{\lambda} a\right)\right] \\
& =\frac{1}{2}\left[2 \mathrm{~s}_{\lambda}\left(\omega, a \oplus_{\lambda} b\right)-\mathrm{s}_{\lambda}\left(\omega, a \ominus_{\lambda} b\right)-\mathrm{s}_{\lambda}\left(\omega, b \ominus_{\lambda} a\right)\right] .
\end{aligned}
$$

Pode-se observar que

$$
\begin{aligned}
\mathrm{s}_{\lambda}\left(\omega, b \ominus_{\lambda} a\right) & =\frac{1}{2 i}\left\{\left[\frac{\mathrm{e}_{\lambda}(b)}{\mathrm{e}_{\lambda}(a)}\right]^{i \omega}-\left[\frac{\mathrm{e}_{\lambda}(b)}{\mathrm{e}_{\lambda}(a)}\right]^{-i \omega}\right\} \\
& =\frac{1}{2 i}\left\{\left[\frac{\mathrm{e}_{\lambda}(a)}{\mathrm{e}_{\lambda}(b)}\right]^{-i \omega}-\left[\frac{\mathrm{e}_{\lambda}(a)}{\mathrm{e}_{\lambda}(b)}\right]^{i \omega}\right\} \\
& =-\frac{1}{2 i}\left\{\left[\frac{\mathrm{e}_{\lambda}(a)}{\mathrm{e}_{\lambda}(b)}\right]^{i \omega}-\left[\frac{\mathrm{e}_{\lambda}(a)}{\mathrm{e}_{\lambda}(b)}\right]^{-i \omega}\right\}
\end{aligned}
$$




$$
=-\mathrm{s}_{\lambda}\left(\omega, a \ominus_{\lambda} b\right) .
$$

Substituindo esse resultado na Eq. (3.51), tem-se:

$$
\mathrm{s}_{\lambda}(\omega, a) \mathrm{c}_{\lambda}(\omega, b)+\mathrm{s}_{\lambda}(\omega, b) \mathrm{c}_{\lambda}(\omega, a)=\mathrm{s}_{\lambda}\left(\omega, a \oplus_{\lambda} b\right) .
$$

Desta forma, verifica-se a validade da propriedade listada na Eq. (3.48c). Procedimento similar pode ser utilizado para demonstração da propriedade apresentada na Eq. (3.48d).

Outra propriedade apresentada no Cap. 5 é a soma de arcos em relação a variável de frequência $\omega$. Essas igualdades, dadas por

$$
\begin{aligned}
& \mathrm{c}_{\lambda}\left(\omega_{1} \pm \omega_{2}, t\right)=\mathrm{c}_{\lambda}\left(\omega_{1}, t\right) \mathrm{c}_{\lambda}\left(\omega_{2}, t\right) \mp \mathrm{s}_{\lambda}\left(\omega_{1}, t\right) \mathrm{s}_{\lambda}\left(\omega_{2}, t\right), \\
& \mathrm{s}_{\lambda}\left(\omega_{1} \pm \omega_{2}, t\right)=\mathrm{s}_{\lambda}\left(\omega_{1}, t\right) \mathrm{c}_{\lambda}\left(\omega_{2}, t\right) \pm \mathrm{s}_{\lambda}\left(\omega_{2}, t\right) \mathrm{c}_{\lambda}\left(\omega_{1}, t\right),
\end{aligned}
$$

podem ser verificadas de forma bem simples. De fato, utilizando nossa generalização da função cosseno, temos:

$$
\begin{aligned}
\mathrm{c}_{\lambda}\left(\omega_{1}+\omega_{2}, t\right) & =\frac{\left[\mathrm{e}_{\lambda}(t)\right]^{i\left(\omega_{1}+\omega_{2}\right)}+\left[\mathrm{e}_{\lambda}(t)\right]^{-i\left(\omega_{1}+\omega_{2}\right)}}{2} \\
& =\frac{\left[\mathrm{e}_{\lambda}(t)\right]^{i \omega_{1}}\left[\mathrm{e}_{\lambda}(t)\right]^{i \omega_{2}}+\left[\mathrm{e}_{\lambda}(t)\right]^{-i \omega_{1}}\left[\mathrm{e}_{\lambda}(t)\right]^{-i \omega_{2}}}{2} .
\end{aligned}
$$

Utilizando a relação de Euler da Eq. (3.41) em cada termo da Eq. (3.56) segue

$$
\begin{aligned}
\mathrm{c}_{\lambda}\left(\omega_{1}\right. & \left.+\omega_{2}, t\right)=\frac{\left[\mathrm{c}_{\lambda}\left(\omega_{1}, t\right)+i \mathrm{~s}_{\lambda}\left(\omega_{1}, t\right)\right]\left[\mathrm{c}_{\lambda}\left(\omega_{2}, t\right)+i \mathrm{~s}_{\lambda}\left(\omega_{2}, t\right)\right]}{2} \\
& +\frac{\left[\mathrm{c}_{\lambda}\left(\omega_{1}, t\right)-i \mathrm{~s}_{\lambda}\left(\omega_{1}, t\right)\right]\left[\mathrm{c}_{\lambda}\left(\omega_{2}, t\right)-i \mathrm{~s}_{\lambda}\left(\omega_{2}, t\right)\right]}{2} \\
& =\frac{\mathrm{c}_{\lambda}\left(\omega_{1}, t\right) \mathrm{c}_{\lambda}\left(\omega_{2}, t\right)+i \mathrm{c}_{\lambda}\left(\omega_{1}, t\right) \mathrm{s}_{\lambda}\left(\omega_{2}, t\right)+i \mathrm{c}_{\lambda}\left(\omega_{2}, t\right) \mathrm{s}_{\lambda}\left(\omega_{1}, t\right)-\mathrm{s}_{\lambda}\left(\omega_{1}, t\right) \mathrm{s}_{\lambda}\left(\omega_{2}, t\right)}{2} \\
& +\frac{\mathrm{c}_{\lambda}\left(\omega_{1}, t\right) \mathrm{c}_{\lambda}\left(\omega_{2}, t\right)-i \mathrm{c}_{\lambda}\left(\omega_{1}, t\right) \mathrm{s}_{\lambda}\left(\omega_{2}, t\right)-i \mathrm{c}_{\lambda}\left(\omega_{2}, t\right) \mathrm{s}_{\lambda}\left(\omega_{1}, t\right)-\mathrm{s}_{\lambda}\left(\omega_{1}, t\right) \mathrm{s}_{\lambda}\left(\omega_{2}, t\right)}{2} \\
& =\frac{2 \mathrm{c}_{\lambda}\left(\omega_{1}, t\right) \mathrm{c}_{\lambda}\left(\omega_{2}, t\right)-2 \mathrm{~s}_{\lambda}\left(\omega_{1}, t\right) \mathrm{s}_{\lambda}\left(\omega_{2}, t\right)}{2} \\
& =c_{\lambda}\left(\omega_{1}, t\right) \mathrm{c}_{\lambda}\left(\omega_{2}, t\right)-\mathrm{s}_{\lambda}\left(\omega_{1}, t\right) \mathrm{s}_{\lambda}\left(\omega_{2}, t\right) .
\end{aligned}
$$

O resultado para $c_{\lambda}\left(\omega_{1}-\omega_{2}, t\right)$ pode ser verificado de modo similar. Ao considerar $\omega_{1}=\omega_{2}$ na Eq. (3.57), obtém-se

$$
\mathrm{c}_{\lambda}\left(\omega_{1}+\omega_{1}, t\right)=\mathrm{c}_{\lambda}\left(2 \omega_{1}, t\right)=\mathrm{c}_{\lambda}^{2}\left(\omega_{1}, t\right)-\mathrm{s}_{\lambda}^{2}\left(\omega_{1}, t\right),
$$

equivalente ao arco duplo nas versões tradicionais. 
Já as propriedades relativas ao seno generalizado podem ser demonstrados fazendo,

$$
\begin{aligned}
\mathrm{s}_{\lambda}\left(\omega_{1}\right. & \left.+\omega_{2}, t\right)=\frac{\left[\mathrm{e}_{\lambda}(t)\right]^{i\left(\omega_{1}+\omega_{2}\right)}-\left[\mathrm{e}_{\lambda}(t)\right]^{-i\left(\omega_{1}+\omega_{2}\right)}}{2 i} \\
& =\frac{\left[\mathrm{e}_{\lambda}(t)\right]^{i \omega_{1}}\left[\mathrm{e}_{\lambda}(t)\right]^{i \omega_{2}}-\left[\mathrm{e}_{\lambda}(t)\right]^{-i \omega_{1}}\left[\mathrm{e}_{\lambda}(t)\right]^{-i \omega_{2}}}{2 i} \\
& =\frac{\left[\mathrm{c}_{\lambda}\left(\omega_{1}, t\right)+i \mathrm{~s}_{\lambda}\left(\omega_{1}, t\right)\right]\left[\mathrm{c}_{\lambda}\left(\omega_{2}, t\right)+i \mathrm{~s}_{\lambda}\left(\omega_{2}, t\right)\right]}{2 i} \\
& -\frac{\left[\mathrm{c}_{\lambda}\left(\omega_{1}, t\right)-i \mathrm{~s}_{\lambda}\left(\omega_{1}, t\right)\right]\left[\mathrm{c}_{\lambda}\left(\omega_{2}, t\right)-i \mathrm{~s}_{\lambda}\left(\omega_{2}, t\right)\right]}{2 i} \\
& =\frac{\mathrm{c}_{\lambda}\left(\omega_{1}, t\right) \mathrm{c}_{\lambda}\left(\omega_{2}, t\right)+i \mathrm{c}_{\lambda}\left(\omega_{1}, t\right) \mathrm{s}_{\lambda}\left(\omega_{2}, t\right)+i \mathrm{c}_{\lambda}\left(\omega_{2}, t\right) \mathrm{s}_{\lambda}\left(\omega_{1}, t\right)-\mathrm{s}_{\lambda}\left(\omega_{1}, t\right) \mathrm{s}_{\lambda}\left(\omega_{2}, t\right)}{2 i} \\
& -\frac{\mathrm{c}_{\lambda}\left(\omega_{1}, t\right) \mathrm{c}_{\lambda}\left(\omega_{2}, t\right)-i \mathrm{c}_{\lambda}\left(\omega_{1}, t\right) \mathrm{s}_{\lambda}\left(\omega_{2}, t\right)-i \mathrm{c}_{\lambda}\left(\omega_{2}, t\right) \mathrm{s}_{\lambda}\left(\omega_{1}, t\right)-\mathrm{s}_{\lambda}\left(\omega_{1}, t\right) \mathrm{s}_{\lambda}\left(\omega_{2}, t\right)}{2 i} \\
& =\frac{2 i \mathrm{c}_{\lambda}\left(\omega_{1}, t\right) \mathrm{s}_{\lambda}\left(\omega_{2}, t\right)+2 i \mathrm{c}_{\lambda}\left(\omega_{2}, t\right) \mathrm{s}_{\lambda}\left(\omega_{1}, t\right)}{2 i} \\
& =\mathrm{c}_{\lambda}\left(\omega_{1}, t\right) \mathrm{s}_{\lambda}\left(\omega_{2}, t\right)+\mathrm{c}_{\lambda}\left(\omega_{2}, t\right) \mathrm{s}_{\lambda}\left(\omega_{1}, t\right) .
\end{aligned}
$$

A demonstração do resultado relativo a $s_{\lambda}\left(\omega_{1}-\omega_{2}, t\right)$ pode ser obtido de modo semelhante. Para $\omega_{1}=\omega_{2}$ na Eq. (3.59), obtemos expressão semelhante ao arco duplo na função seno tradicional,

$$
\mathrm{s}_{\lambda}\left(\omega_{1}+\omega_{1}, t\right)=\mathrm{s}_{\lambda}\left(2 \omega_{1}, t\right)=2 \mathrm{~s}_{\lambda}\left(\omega_{1}, t\right) \mathrm{c}_{\lambda}\left(\omega_{1}, t\right) .
$$

Para demonstrar os resultados apresentados nas Eq. (5.57), fazemos uso do seguinte resultado:

$$
\begin{aligned}
{\left[\mathrm{e}_{\lambda}(t)\right]^{n} } & =(1+\lambda t)^{\frac{n}{\lambda}} \\
& =\left[1+\left(\frac{\lambda}{n}\right) n t\right]^{\frac{n}{\lambda}} \\
& =\mathrm{e}_{\lambda / n}(n t) .
\end{aligned}
$$

De forma equivalente, pode-se escrever,

$$
\mathrm{e}_{\lambda}(n t)=\left[\mathrm{e}_{\lambda n}(t)\right]^{n}
$$

Utilizando a Eq. (3.62), pode-se escrever

$$
\begin{aligned}
\mathrm{c}_{\lambda}(\omega, n t) & =\frac{\left[\mathrm{e}_{\lambda}(n t)\right]^{i \omega}+\left[\mathrm{e}_{\lambda}(n t)\right]^{-i \omega}}{2} \\
& =\frac{\left[\mathrm{e}_{\lambda n}(t)\right]^{i n \omega}+\left[\mathrm{e}_{\lambda n}(t)\right]^{-i n \omega}}{2} \\
& =c_{n \lambda}(n \omega, t) .
\end{aligned}
$$


De modo similar prova-se que $\mathrm{s}_{\lambda}(\omega, n t)=\mathrm{s}_{n \lambda}(n \omega, t)$.

\subsection{Limites fundamentais}

Uma propriedade muito utilizada para simplificação na trigonometria tradicional é o limite fundamental. Também na versão generalizada mantemos esse resultado. Para confirmá-lo podemos utilizar o teorema de L'Hospital, já que

$$
\lim _{t \rightarrow 0} \frac{\mathrm{s}_{\lambda}(\omega, t)}{t}=\frac{\lim _{t \rightarrow 0} \mathrm{~s}_{\lambda}(\omega, t)}{\lim _{t \rightarrow 0} t}=\frac{a}{b},
$$

e, no limite, $a=b=0$. Desta forma,

$$
\lim _{t \rightarrow 0} \frac{\mathrm{s}_{\lambda}(\omega, t)}{t}=\lim _{t \rightarrow 0} \frac{d / d t \mathrm{~s}_{\lambda}(\omega, t)}{d / d t t}=\lim _{t \rightarrow 0} \frac{\omega(1+\lambda t)^{-1} \mathrm{c}_{\lambda}(\omega, t)}{1} .
$$

Fazendo o limite de $c_{\lambda}(\omega, t)$ com $t \rightarrow 0$, tem-se:

$$
\lim _{t \rightarrow 0} c_{\lambda}(\omega, t)=\lim _{t \rightarrow 0} \frac{\left[\mathrm{e}_{\lambda}(t)\right]^{i \omega}+\left[\mathrm{e}_{\lambda}(t)\right]^{-i \omega}}{2}=\frac{1+1}{2}=1 .
$$

Utilizando este resultado na Eq. (3.65), tem-se

$$
\lim _{t \rightarrow 0} \frac{\mathrm{s}_{\lambda}(\omega, t)}{t}=\omega(1+\lambda 0)^{-1} 1=\omega .
$$

Desta forma, demonstra-se o resultado apresentado na Eq. (5.66).

O limite fundamental relativo à variável de frequência $\omega$, pode ser obtido de forma semelhante, já que

$$
\lim _{\omega \rightarrow 0} \mathrm{~s}_{\lambda}(\omega, t)=\lim _{\omega \rightarrow 0} \frac{\left[\mathrm{e}_{\lambda}(t)\right]^{i \omega}-\left[\mathrm{e}_{\lambda}(t)\right]^{-i \omega}}{2 i}=0 .
$$

Ao calcular $\lim _{\omega \rightarrow 0} \mathrm{~s}_{\lambda}(\omega, t) / \omega=a / b$ em que, no limite $a=b=0$, pode-se utilizar o teorema de L'Hospital, de forma que

$$
\lim _{\omega \rightarrow 0} \frac{\mathrm{s}_{\lambda}(\omega, t)}{\omega}=\lim _{\omega \rightarrow 0} \frac{d / d \omega \mathrm{s}_{\lambda}(\omega, t)}{d / d \omega \omega}=\lim _{\omega \rightarrow 0} \frac{\ln \left[\mathrm{e}_{\lambda}(t)\right] \mathrm{c}_{\lambda}(\omega, t)}{1} .
$$

Calculando

$$
\lim _{\omega \rightarrow 0} \mathrm{c}_{\lambda}(\omega, t)=\frac{\left[\mathrm{e}_{\lambda}(t)\right]^{i 0}+\left[\mathrm{e}_{\lambda}(t)\right]^{-i 0}}{2}=\frac{1+1}{2}=1
$$

temos

$$
\lim _{\omega \rightarrow 0} \frac{\mathrm{s}_{\lambda}(\omega, t)}{\omega}=\frac{\ln \left[\mathrm{e}_{\lambda}(t)\right] 1}{1}=\ln \left[\mathrm{e}_{\lambda}(t)\right] .
$$

Isso valida a propriedade apresentada na Eq. (5.67) do Cap. 5. 


\subsection{Relação fundamental das funções hiperbólicas}

Nas funções hiperbólicas usuais, um resultado muito importante é a relação fundamental, dada por $\cosh ^{2}(\omega t)-\operatorname{senh}^{2}(\omega t)=1$. Essa relação também é válida para nossas funções hiperbólicas generalizadas, já que

$$
\begin{aligned}
\operatorname{ch}_{\lambda}^{2}(\omega, t)-\operatorname{sh}_{\lambda}^{2}(\omega, t) & =\left\{\frac{\left[\mathrm{e}_{\lambda}(t)\right]^{\omega}+\left[\mathrm{e}_{\lambda}(t)\right]^{-\omega}}{2}\right\}^{2}-\left\{\frac{\left[\mathrm{e}_{\lambda}(t)\right]^{\omega}-\left[\mathrm{e}_{\lambda}(t)\right]^{-\omega}}{2}\right\}^{2} \\
& =\frac{\left[\mathrm{e}_{\lambda}(t)\right]^{2 \omega}+2+\left[\mathrm{e}_{\lambda}(t)\right]^{-2 \omega}-\left[\mathrm{e}_{\lambda}(t)\right]^{2 \omega}+2-\left[\mathrm{e}_{\lambda}(t)\right]^{-2 \omega}}{4} \\
& =\frac{2+2}{4}=1 .
\end{aligned}
$$

Isso confirma o resultado apresentado na Eq. (5.83) do Cap. 5.

\subsection{Produtos de funções hiperbólicas generalizadas}

A exemplo das funções trigonométricas, existem importantes resultados relacionados aos produtos das funções hiperbólicas generalizadas que podem ser úteis em cálculos e na simplificação de resultados. Seguem os produtos de funções com variável de frequência $\omega$ e diferentes valores no argumento principal

a-) Produto de cossenos hiperbólicos

$$
\begin{aligned}
\operatorname{ch}_{\lambda}(\omega, a) & \operatorname{ch}_{\lambda}(\omega, b)=\left\{\frac{\left[\mathrm{e}_{\lambda}(a)\right]^{\omega}+\left[\mathrm{e}_{\lambda}(a)\right]^{-\omega}}{2}\right\}\left\{\frac{\left[\mathrm{e}_{\lambda}(b)\right]^{\omega}+\left[\mathrm{e}_{\lambda}(b)\right]^{-\omega}}{2}\right\} \\
= & \frac{1}{4}\left\{\left[\mathrm{e}_{\lambda}(a) \mathrm{e}_{\lambda}(b)\right]^{\omega}+\left[\frac{\mathrm{e}_{\lambda}(a)}{\mathrm{e}_{\lambda}(b)}\right]^{\omega}+\left[\frac{\mathrm{e}_{\lambda}(a)}{\mathrm{e}_{\lambda}(b)}\right]^{-\omega}+\left[\mathrm{e}_{\lambda}(a) \mathrm{e}_{\lambda}(b)\right]^{-\omega}\right\} \\
= & \frac{1}{4}\left\{\left[\mathrm{e}_{\lambda}\left(a \oplus_{\lambda} b\right)\right]^{\omega}+\left[\mathrm{e}_{\lambda}\left(a \ominus_{\lambda} b\right)\right]^{\omega}+\left[\mathrm{e}_{\lambda}\left(a \ominus_{\lambda} b\right)\right]^{-\omega}+\left[\mathrm{e}_{\lambda}\left(a \oplus_{\lambda} b\right)\right]^{-\omega}\right\} \\
= & \frac{1}{2}\left\{\frac{\left.\mathrm{e}_{\lambda}\left(a \oplus_{\lambda} b\right)\right]^{\omega}+\left[\mathrm{e}_{\lambda}\left(a \oplus_{\lambda} b\right)\right]^{-\omega}+\left[\mathrm{e}_{\lambda}\left(a \ominus_{\lambda} b\right)\right]^{\omega}+\left[\mathrm{e}_{\lambda}\left(a \ominus_{\lambda} b\right)\right]^{-\omega}}{2}\right\} \\
= & \frac{1}{2}\left[\operatorname{ch}_{\lambda}\left(\omega, a \oplus_{\lambda} b\right)+\operatorname{ch}_{\lambda}\left(\omega, a \ominus_{\lambda} b\right)\right] .
\end{aligned}
$$

b-) Produto de senos hiperbólicos

$$
\begin{aligned}
& \operatorname{sh}_{\lambda}(\omega, a) \operatorname{sh}_{\lambda}(\omega, b)=\left\{\frac{\left[\mathrm{e}_{\lambda}(a)\right]^{\omega}-\left[\mathrm{e}_{\lambda}(a)\right]^{-\omega}}{2}\right\}\left\{\frac{\left[\mathrm{e}_{\lambda}(b)\right]^{\omega}-\left[\mathrm{e}_{\lambda}(b)\right]^{-\omega}}{2}\right\} \\
& =-\frac{1}{4}\left\{\left[\mathrm{e}_{\lambda}(a) \mathrm{e}_{\lambda}(b)\right]^{\omega}-\left[\frac{\mathrm{e}_{\lambda}(a)}{\mathrm{e}_{\lambda}(b)}\right]^{\omega}-\left[\frac{\mathrm{e}_{\lambda}(a)}{\mathrm{e}_{\lambda}(b)}\right]^{-\omega}+\left[\mathrm{e}_{\lambda}(a) \mathrm{e}_{\lambda}(b)\right]^{-\omega}\right\}
\end{aligned}
$$




$$
\begin{aligned}
& =\frac{1}{4}\left\{\left[\mathrm{e}_{\lambda}\left(a \oplus_{\lambda} b\right)\right]^{\omega}-\left[\mathrm{e}_{\lambda}\left(a \ominus_{\lambda} b\right)\right]^{\omega}-\left[\mathrm{e}_{\lambda}\left(a \ominus_{\lambda} b\right)\right]^{-\omega}+\left[\mathrm{e}_{\lambda}\left(a \oplus_{\lambda} b\right)\right]^{-\omega}\right\} \\
& =\frac{1}{2}\left\{\frac{\left.\mathrm{e}_{\lambda}\left(a \oplus_{\lambda} b\right)\right]^{\omega}+\left[\mathrm{e}_{\lambda}\left(a \oplus_{\lambda} b\right)\right]^{-\omega}-\left[\mathrm{e}_{\lambda}\left(a \ominus_{\lambda} b\right)\right]^{\omega}-\left[\mathrm{e}_{\lambda}\left(a \ominus_{\lambda} b\right)\right]^{-\omega}}{2}\right\} \\
& =\frac{1}{2}\left[\operatorname{ch}_{\lambda}\left(\omega, a \oplus_{\lambda} b\right)-\operatorname{ch}_{\lambda}\left(\omega, a \ominus_{\lambda} b\right)\right] .
\end{aligned}
$$

c-) Produto de senos hiperbólicos por cossenos hiperbólicos

$$
\begin{aligned}
\operatorname{sh}_{\lambda}(\omega, a) & \operatorname{ch}_{\lambda}(\omega, b)=\left\{\frac{\left[\mathrm{e}_{\lambda}(a)\right]^{\omega}-\left[\mathrm{e}_{\lambda}(a)\right]^{-\omega}}{2}\right\}\left\{\frac{\left[\mathrm{e}_{\lambda}(b)\right]^{\omega}+\left[\mathrm{e}_{\lambda}(b)\right]^{-\omega}}{2}\right\} \\
= & \frac{1}{4}\left\{\left[\mathrm{e}_{\lambda}(a) \mathrm{e}_{\lambda}(b)\right]^{\omega}+\left[\frac{\mathrm{e}_{\lambda}(a)}{\mathrm{e}_{\lambda}(b)}\right]^{\omega}-\left[\frac{\mathrm{e}_{\lambda}(a)}{\mathrm{e}_{\lambda}(b)}\right]^{-\omega}-\left[\mathrm{e}_{\lambda}(a) \mathrm{e}_{\lambda}(b)\right]^{-\omega}\right\} \\
= & \frac{1}{4}\left\{\left[\mathrm{e}_{\lambda}\left(a \oplus_{\lambda} b\right)\right]^{\omega}+\left[\mathrm{e}_{\lambda}\left(a \ominus_{\lambda} b\right)\right]^{\omega}-\left[\mathrm{e}_{\lambda}\left(a \ominus_{\lambda} b\right)\right]^{-\omega}-\left[\mathrm{e}_{\lambda}\left(a \oplus_{\lambda} b\right)\right]^{-\omega}\right\} \\
= & \frac{1}{2}\left\{\frac{\left.\mathrm{e}_{\lambda}\left(a \oplus_{\lambda} b\right)\right]^{\omega}-\left[\mathrm{e}_{\lambda}\left(a \oplus_{\lambda} b\right)\right]^{-\omega}+\left[\mathrm{e}_{\lambda}\left(a \ominus_{\lambda} b\right)\right]^{\omega}-\left[\mathrm{e}_{\lambda}\left(a \ominus_{\lambda} b\right)\right]^{-\omega}}{2 i}\right\} \\
= & \frac{1}{2}\left[\operatorname{sh}_{\lambda}\left(\omega, a \oplus_{\lambda} b\right)-\operatorname{sh}_{\lambda}\left(\omega, a \ominus_{\lambda} b\right)\right] .
\end{aligned}
$$

Desta forma, validamos as propriedades listadas nas Eq. (5.85) do Cap. 5. Já nas Eq. (5.86) tem-se propriedades referentes a produtos de funções hiperbólicas generalizadas na variável principal $t$, com diferentes frequências. Neste caso, a demonstração pode ser feita,

a-) Produto de cossenos hiperbólicos

$$
\begin{aligned}
\operatorname{ch}_{\lambda}\left(\omega_{1}, t\right) & \operatorname{ch}_{\lambda}\left(\omega_{2}, t\right)=\left\{\frac{\left[\mathrm{e}_{\lambda}(t)\right]^{\omega_{1}}+\left[\mathrm{e}_{\lambda}(t)\right]^{-\omega_{1}}}{2}\right\}\left\{\frac{\left.\mathrm{e}_{\lambda}(t)\right]^{\omega_{2}}+\left[\mathrm{e}_{\lambda}(t)\right]^{-\omega_{2}}}{2}\right\} \\
= & \frac{1}{4}\left\{\left[\mathrm{e}_{\lambda}(t)\right]^{\left(\omega_{1}+\omega_{2}\right)}+\left[\mathrm{e}_{\lambda}(t)\right]^{\left(\omega_{1}-\omega_{2}\right)}+\left[\mathrm{e}_{\lambda}(t)\right]^{-\left(\omega_{1}-\omega_{2}\right)}+\left[\mathrm{e}_{\lambda}(t)\right]^{-\left(\omega_{1}+\omega_{2}\right)}\right\} \\
= & \frac{1}{4}\left\{\frac{\left[\mathrm{e}_{\lambda}(t)\right]^{\left(\omega_{1}+\omega_{2}\right)}+\left[\mathrm{e}_{\lambda}(t)\right]^{-\left(\omega_{1}+\omega_{2}\right)}+\left[\mathrm{e}_{\lambda}(t)\right]^{\left(\omega_{1}-\omega_{2}\right)}+\left[\mathrm{e}_{\lambda}(t)\right]^{-\left(\omega_{1}-\omega_{2}\right)}}{2}\right\} \\
= & \frac{1}{2}\left[\operatorname{ch}_{\lambda}\left(\omega_{1}+\omega_{2}, t\right)+\operatorname{ch}_{\lambda}\left(\omega_{1}-\omega_{2}, t\right)\right] .
\end{aligned}
$$

b-) Produto de senos hiperbólicos

$$
\begin{aligned}
\operatorname{sh}_{\lambda}\left(\omega_{1}, t\right) & \operatorname{sh}_{\lambda}\left(\omega_{2}, t\right)=\left\{\frac{\left[\mathrm{e}_{\lambda}(t)\right]^{\omega_{1}}-\left[\mathrm{e}_{\lambda}(t)\right]^{-\omega_{1}}}{2}\right\}\left\{\frac{\left.\mathrm{e}_{\lambda}(t)\right]^{\omega_{2}}-\left[\mathrm{e}_{\lambda}(t)\right]^{-\omega_{2}}}{2}\right\} \\
= & \frac{1}{4}\left\{\left[\mathrm{e}_{\lambda}(t)\right]^{\left(\omega_{1}+\omega_{2}\right)}-\left[\mathrm{e}_{\lambda}(t)\right]^{\left(\omega_{1}-\omega_{2}\right)}-\left[\mathrm{e}_{\lambda}(t)\right]^{-\left(\omega_{1}-\omega_{2}\right)}+\left[\mathrm{e}_{\lambda}(t)\right]^{-\left(\omega_{1}+\omega_{2}\right)}\right\} \\
= & \frac{1}{2}\left\{\frac{\left[\mathrm{e}_{\lambda}(t)\right]^{\left(\omega_{1}+\omega_{2}\right)}+\left[\mathrm{e}_{\lambda}(t)\right]^{-\left(\omega_{1}+\omega_{2}\right)}-\left[\mathrm{e}_{\lambda}(t)\right]^{\left(\omega_{1}-\omega_{2}\right)}-\left[\mathrm{e}_{\lambda}(t)\right]^{-\left(\omega_{1}-\omega_{2}\right)}}{2}\right\}
\end{aligned}
$$




$$
=-\frac{1}{2}\left[\operatorname{ch}_{\lambda}\left(\omega_{1}+\omega_{2}, t\right)-\operatorname{ch}_{\lambda}\left(\omega_{1}-\omega_{2}, t\right)\right]
$$

c-) Produto de senos hiperbólicos por cossenos hiperbólicos

$$
\begin{aligned}
\operatorname{sh}_{\lambda}\left(\omega_{1}, t\right) & \operatorname{ch}_{\lambda}\left(\omega_{2}, t\right)=\left\{\frac{\left[\mathrm{e}_{\lambda}(t)\right]^{\omega_{1}}-\left[\mathrm{e}_{\lambda}(t)\right]^{-\omega_{1}}}{2}\right\}\left\{\frac{\left[\mathrm{e}_{\lambda}(t)\right]^{\omega_{2}}+\left[\mathrm{e}_{\lambda}(t)\right]^{-\omega_{2}}}{2}\right\} \\
= & \frac{1}{4}\left\{\left[\mathrm{e}_{\lambda}(t)\right]^{\left(\omega_{1}+\omega_{2}\right)}+\left[\mathrm{e}_{\lambda}(t)\right]^{\left(\omega_{1}-\omega_{2}\right)}-\left[\mathrm{e}_{\lambda}(t)\right]^{-\left(\omega_{1}-\omega_{2}\right)}-\left[\mathrm{e}_{\lambda}(t)\right]^{-\left(\omega_{1}+\omega_{2}\right)}\right\} \\
= & \frac{1}{2}\left\{\frac{\left[\mathrm{e}_{\lambda}(t)\right]^{\left(\omega_{1}+\omega_{2}\right)}-\left[\mathrm{e}_{\lambda}(t)\right]^{-\left(\omega_{1}+\omega_{2}\right)}+\left[\mathrm{e}_{\lambda}(t)\right]^{\left(\omega_{1}-\omega_{2}\right)}-\left[\mathrm{e}_{\lambda}(t)\right]^{-\left(\omega_{1}-\omega_{2}\right)}}{2}\right\} \\
= & \frac{1}{2}\left[\operatorname{sh}_{\lambda}\left(\omega_{1}+\omega_{2}, t\right)-\operatorname{sh}_{\lambda}\left(\omega_{1}-\omega_{2}, t\right)\right] .
\end{aligned}
$$

Nestes termos, confirmamos as igualdades apresentadas nas Eq. (5.59).

\subsection{Relação entre funções trigonométricas e hiperbólicas}

Um importante resultado apresentado nas Eq. (5.93) do Cap. 5 são as relações entre as funções trigonométricas e hiperbólicas quando permite-se parâmetros complexos. Para verificar estes resultados considera-se, inicialmente, a igualdade $\mathrm{e}_{\lambda / n}(n t)=\left[\mathrm{e}_{\lambda}(t)\right]^{n}$, já demonstrada na Eq. (3.61) deste Apêndice. Fazendo $\mathrm{e}_{k / i}(i t)=\left[\mathrm{e}_{k}(t)\right]^{i}$, tem-se que, se $\lambda=k / i$, então, no final, tem-se que $\mathrm{e}_{\lambda}(i t)=\left[\mathrm{e}_{i \lambda}(t)\right]^{i}$. Desta forma, pode-se escrever:

$$
\begin{aligned}
\mathrm{c}_{\lambda}(\omega, i t) & =\frac{\left[\mathrm{e}_{\lambda}(i t)\right]^{i \omega}+\left[\mathrm{e}_{\lambda}(i t)\right]^{-i \omega}}{2} \\
& =\frac{\left\{\left[\mathrm{e}_{i \lambda}(t)\right]^{i \omega}\right\}^{i}+\left\{\left[\mathrm{e}_{i \lambda}(t)\right]^{-i \omega}\right\}^{i}}{2} \\
& =\frac{\left[\mathrm{e}_{i \lambda}(t)\right]^{-\omega}+\left[\mathrm{e}_{i \lambda}(t)\right]^{\omega}}{2} \\
& =\operatorname{ch}_{i \lambda}(\omega, t) .
\end{aligned}
$$

De modo semelhante pode-se escrever

$$
\begin{aligned}
\operatorname{ch}_{\lambda}(\omega, i t) & =\frac{\left[\mathrm{e}_{\lambda}(i t)\right]^{\omega}+\left[\mathrm{e}_{\lambda}(i t)\right]^{-\omega}}{2} \\
& =\frac{\left\{\left[\mathrm{e}_{i \lambda}(t)\right]^{\omega}\right\}^{i}+\left\{\left[\mathrm{e}_{i \lambda}(t)\right]^{-\omega}\right\}^{i}}{2} \\
& =\frac{\left[\mathrm{e}_{i \lambda}(t)\right]^{i \omega}+\left[\mathrm{e}_{i \lambda}(t)\right]^{-i \omega}}{2} \\
& =c_{i \lambda}(\omega, t) .
\end{aligned}
$$


Demonstração similar pode ser feita tanto para mostrar que $\mathrm{s}_{\lambda}(\omega, i t)=i \operatorname{sh}_{i \lambda}(\omega, t)$ como também $\operatorname{sh}_{\lambda}(\omega, i t)=i \mathrm{~s}_{i \lambda}(\omega, t)$.

Essa relação pode ainda ser feita pela variável de frequência $\omega$. Neste caso,

$$
\begin{aligned}
\mathrm{c}_{\lambda}(i \omega, t) & =\frac{\left[\mathrm{e}_{\lambda}(t)\right]^{i(i \omega)}+\left[\mathrm{e}_{\lambda}(t)\right]^{-i(i \omega)}}{2} \\
& =\frac{\left[\mathrm{e}_{\lambda}(t)\right]^{-\omega}+\left[\mathrm{e}_{\lambda}(t)\right]^{\omega}}{2} \\
& =\operatorname{ch}_{\lambda}(\omega, t) .
\end{aligned}
$$

De modo análogo mostra-se que $\operatorname{ch}_{\lambda}(i \omega, t)=\mathrm{c}_{\lambda}(\omega, t)$. Para verificar o resultado que envolve $\mathrm{s}_{\lambda}$ e $\mathrm{sh}_{\lambda}$ pode-se calcular

$$
\begin{aligned}
\mathrm{s}_{\lambda}(i \omega, t) & =\frac{\left[\mathrm{e}_{\lambda}(t)\right]^{i(i \omega)}-\left[\mathrm{e}_{\lambda}(t)\right]^{-i(i \omega)}}{2 i} \\
& =\frac{\left[\mathrm{e}_{\lambda}(t)\right]^{-\omega}-\left[\mathrm{e}_{\lambda}(t)\right]^{\omega}}{2 i} \\
& =i \operatorname{sh}_{\lambda}(\omega, t) .
\end{aligned}
$$

A verificação da igualdade $\operatorname{sh}_{\lambda}(i \omega, t)=i \mathrm{~s}_{\lambda}(\omega, t)$ segue as mesmas diretrizes dos casos anteriores. Deste modo, verificamos a validade das Eq. (5.93) e Eq. (5.96) do Cap. 5.

Neste apêndice omitimos demonstrações já feitas no decorrer do texto e cálculos diretos, como por exemplo, os das integrais e derivadas das funções trigonométricas e hiperbólicas generalizados. Tais resultados são obtidos mediante o cálculo direto da expressão sem necessidade de nenhuma técnica diferenciada. 


\section{Referências $^{1}$}

[1] F. Bombal, "Laurent Schwartz, el matemático que quería cambiar el mundo.", Gaceta de la Real Sociedad Matemática Española, vol. 6, no. 1, pp. 177-201, 2003. 1

[2] C. Tsallis, "Possible generalization of boltzmann-gibbs statistics", J. Stat. Phys., vol. 52, no. 1, pp. 479-487, 1988. 1, 3, 5, 6, 37, 97

[3] A. S. Martinez, R. S. González e A. L. Espíndola, "Generalized exponential function and discrete growth models", Physica A: Statistical Mechanics and its Applications, vol. 388, no. 14, pp. 2922 - 2930, 2009. 1

[4] B. C. T. Cabella, A. S. Martinez e F. Ribeiro, "Data collapse, scaling functions, and analytical solutions of generalized growth models", Phys. Rev. E, vol. 83, p. 061902, Jun 2011. 1, 20

[5] L. S. dos Santos, N. Destefano e A. S. Martinez, "Decision making generalized by a cumulative probability weighting function", Physica A: Statistical Mechanics and its Applications, vol. 490, pp. 250 - 259, 2018. 1

[6] N. Destefano e A. S. Martinez, "The additive property of the inconsistency degree in intertemporal decision making through the generalization of psychophysical laws", Physica A: Statistical Mechanics and its Applications, vol. 390, no. 10 , pp. $1763-1772,2011.1$

[7] O. Menin, A. Martinez e A. Costa, "Reconstruction of bremsstrahlung spectra from attenuation data using generalized simulated annealing", Applied Radiation and Isotopes, vol. 111, pp. $80-85,2016.1$

\footnotetext{
${ }^{1}$ De acordo com o estilo LMSC (baseado no estilo IEEE Transactions - Electrical and Electronics Engineers).
} 
[8] R. S. González, A. S. Martinez e C. A. S. Terçariol, "Generalized probability functions", Advances in Mathematical Physics, vol. 2009, Oct 2009. 2

[9] G. Kaniadakis, M. Lissia e A. M. Scarfone, "Two-parameter deformations of logarithm, exponential, and entropy: A consistent framework for generalized statistical mechanics", Phys. Rev. E, vol. 71, p. 046128, Apr 2005. 2

[10] G. Kaniadakis, "Statistical mechanics in the context of special relativity. ii.", Phys. Rev. E, vol. 72, p. 036108, Sep 2005.

[11] V. Schwämmle e C. Tsallis., "Two-parameter generalization of the logarithm and exponential functions and boltzmann-gibbs-shannon entropy", Journal of Mathematical Physics, vol. 48, p. 113301, Nov 2007. 2

[12] M. S. Stanković, S. D. Marinković e P. M. Rajković, "The deformed exponential functions of two variables in the context of various statistical mechanics", Applied Mathematics and Computation, vol. 218, no. 6, pp. 2439 - 2448, 2011. 2

[13] S. D. Marinković, P. M. Rajković e M. S. Stanković, "Deformed exponentials, operators and modeling population growth", Ser. A: Appl. Math. Inform. and Mech., vol. 5, no. 2, pp. 69-77, 2013. 2

[14] T. J. Arruda, R. S. González, C. A. S. Terçariol e A. S. Martinez, "Arithmetical and geometrical means of generalized logarithmic and exponential functions: Generalized sum and product operators", Physics Letters A, vol. 372, no. 15, pp. $2578-2582,2008$. 2, 6

[15] T. C. P. Lobão, P. G. S. Cardoso, S. T. R. Pinho e E. P. Borges, "Some properties of deformed q-numbers", Brazilian Journal of Physics, vol. 39, pp. 402 - 407, 08 2009. 3, 16, 17, 42

[16] N. Kalogeropoulos, "Distributivity and deformation of the reals from Tsallis entropy", Physica A: Statistical Mechanics and its Applications, vol. 391, no. 4, pp. $1120-1127,2012$.

[17] N. Kalogeropoulos, "Tsallis entropy induced metrics and cat(k) spaces", Physica A: Statistical Mechanics and its Applications, vol. 391, no. 12, pp. 3435 - 3445, 2012. 16,42 
[18] N. Kalogeropoulos, "The $\tau_{q}$-fourier transform: Covariance and uniqueness", Modern Physics Letters B, vol. 32, no. 14, p. 1850149, 2018. 3, 16, 24, 42

[19] G. E. Box e D. R. Cox, "An analysis of transformations", Journal of the Royal Statistical Society. Series B (Methodological), vol. 26, no. 2, pp. 211-252, 1964. $3,5,29,30,31,38$

[20] R. M. Sakia, "The Box-Cox transformation technique: A review", Journal of the Royal Statistical Society. Series D (The Statistician), vol. 41, no. 2, pp. 169178, 1992. 3, 29, 38

[21] T. Daimon, Box-Cox Transformation, pp. 176-178. Berlin, Heidelberg: Springer Berlin Heidelberg, 2011. 3, 29

[22] I.-K. Yeo e R. A. Johnson, "A new family of power transformations to improve normality or symmetry", Biometrika, vol. 87, no. 4, pp. 954-959, 2000. 3, 31, 34

[23] G. M. Nakamura, A. H. de Martini e A. S. Martinez, "Extension of inverse $q$-fourier transform via conformal mapping", Physica A: Statistical Mechanics and its Applications, vol. 524, pp. 106 - 111, 2019. 4, 37, 66, 70, 98

[24] D. Sornette, A. Johansen e J.-P. Bouchaud, "Stock market crashes, precursors and replicas", J. Phys. I France, vol. 6, pp. 167-175, 6 1996. 4, 46, 59, 66

[25] J. A. Feigenbaum e P. G. Freund, "Discrete scale invariance in stock markets before crashes", International Journal of Modern Physics B, vol. 10, no. 27, pp. $3737-3745,1996.4,59$

[26] G. Ouillon e D. Sornette, "The concept of 'critical earthquakes' applied to mine rockbursts with time-to-failure analysis", Geophysical Journal International, vol. 143, pp. 454-468, 11 2000. 4, 66

[27] Y. Huang, A. Johansen, M. W. Lee, H. Saleur e D. Sornette, "Artifactual logperiodicity in finite size data: Relevance for earthquake aftershocks", Journal of Geophysical Research: Solid Earth, vol. 105, no. B11, pp. 25451-25471, 2000. 
[28] C. Juliao, I. Gleria, S. Cavalcanti e G. Viswanathan, "Precursores log-periódicos de eventos catastróficos: a quebra de 1999 como exemplo ilustrativo", Revista Brasileira de Ensino de Fisica, vol. 30, pp. 2303.1 - 2303.10, 00 2008. 4, 66

[29] G. Wilk e Z. Włodarczyk, "Tsallis distribution with complex nonextensivity parameter q", Physica A: Statistical Mechanics and its Applications, vol. 413, pp. $53-58,2014.4,66$

[30] G. Wilk e Z. Włodarczyk, "Tsallis distribution decorated with log-periodic oscillation", Entropy, vol. 17, no. 1, pp. 384-400, 2015. 4, 66

[31] E. P. Borges, "On aq-generalization of circular and hyperbolic functions", Journal of Physics A: Mathematical and General, vol. 31, pp. 5281-5288, jun 1998. $4,24,65,67,79,80,85,90,98$

[32] R. S. Gonzalez, Funções generalizadas, modelos de crescimento contínuos e discretos e caminhadas estocásticas em meios desordenados. Tese de Doutorado, Faculdade de Filosofia, Ciências e Letras de Ribeirão Preto, Universidade de São Paulo, 2011. 6, 8, 17, 67, 69

[33] R. S. González, "Difusão anômala: transição entre os regimes localizado e estendido na caminhada do turista unidimensional", Dissertação de Mestrado, Faculdade de Filosofia, Ciências e Letras de Ribeirão Preto, Universidade de São Paulo, Ribeirão Preto, 2006. 8

[34] M. Abramowitz e I. A. Stegun, Handbook of Mathematical Functions. Dover Publications, 9 ed., 1972. 8, 12

[35] S. H. Strogatz, Nonlinear dynamics and chaos. New York: Perseus Book, 1 ed., 1994. 11

[36] H. Eves, Introdução à História da Matemática. Campinas: Unicamp, 1 ed., 2004. $12,60,84$

[37] J. C. V. Sampaio, Notas de aula de Estruturas Algébricas. Departamento de Matemática, Universidade Federal de São Carlos, https://www.dm.ufscar.br/profs/sampaio/algebra.html, data da consulta: 12/03/2020. 12 
[38] E. P. Borges, "A possible deformed algebra and calculus inspired in nonextensive thermostatistics", Physica A, vol. 340, pp. 95-101, 2004. 14, 15, 16

[39] L. Nivanen, A. L. Méhauté e Q. Wang, "Generalized algebra within a nonextensive statistics", Reports on Mathematical Physics, vol. 52, no. 3, pp. 437 444, 2003. 14, 15, 16

[40] W. E. Boyce e R. c. DiPrima, Equações diferenciais elementares e problemas de valores de contorno. Rio de Janeiro: LTC, 10 ed., 2012. 21

[41] G. Sicuro e C. Tsallis, " $q$-generalized representation of the $d$-dimensional dirac delta and q-fourier transform", Physics Letters A, vol. 381, no. 32, pp. 2583 2587, 2017. 24, 25, 41, 45

[42] M. Jauregui e C. Tsallis, "q-generalization of the inverse fourier transform", Physics Letters A, vol. 375, no. 21, pp. 2085 - 2088, 2011. 24, 41, 45

[43] J. W. Brow e R. V. Churchill, Complex Variables and Applications. New York: McGraw-Hill Education, 9 ed., 2013. 24, 44

[44] J. Aitchison, "The statistical analysis of compositional data", Journal of the Royal Statistical Society. Series B (Methodological), vol. 44, no. 2, pp. 139-177, 1982. 29

[45] J. W. Ascombe, F. J.; Tukey, "The criticism of transformations", American Statistical Association and the Biometric Society, 09 1952. 30

[46] J. W. Moore, P. G.; Tukey, "Answer to query no 112", Biometrics, vol. 10, pp. $562-568,1954.30$

[47] J. W. Tukey, "On the comparative anatomy of transformations", Ann. Math. Statist., vol. 28, pp. 602-632, 09 1957. 30

[48] S. A. F. M., "George edward pelham box. 10 october 1919 - 28 march 2013", Biographical Memoirs of Fellows of the Royal Society, vol. 61, pp. 23-37, 2015. 30

[49] J. M. P. Memória, Breve História da Estatística. Brasília: Embrapa Informação Tecnológica, 1 ed., 2004. 31 
[50] D. Scott, Box-Cox Transformations. Rice University (Lead Developer), University of Houston Clear Lake, and Tufts University, http://onlinestatbook.com/2/transformations/box-cox.html, data da consulta: $31 / 01 / 2017.31$

[51] G. Milone, Estatística geral e aplicada. São Paulo: Thompson, 1 ed., 2004. 32

[52] B. F. J. Manly, "Exponential data transformations", Journal of the Royal Statistical Society. Series D (The Statistician), vol. 25, no. 1, pp. 37-42, 1976. 33

[53] J. A. John e N. R. Draper, "An alternative family of transformations", Journal of the Royal Statistical Society. Series C (Applied Statistics), vol. 29, no. 2, pp. 190-197, 1980. 33

[54] P. J. Bickel e K. A. Doksum, "An analysis of transformations revisited", Journal of the American Statistical Association, vol. 76, no. 374, pp. 296-311, 1981. 33

[55] G. G. V. Douglas C. Montgomery, Elizabeth A. Peck, Introduction to linear Analysis. Wiley, 5 ed., 2012. 34

[56] J. J. Spitzer, "A primer on box-cox estimation", The Review of Economics and Statistics, vol. 64, no. 2, pp. 307-313, 1982. 35

[57] G. N. de Souza Miranda, Transformação de Box-Cox e Escores de Blom para correção da heterogeneidade de variâncias do peso de bovinos. Tese de Doutorado, UFV - Universidade Federeal de Viçosa, 2013. 36

[58] T. Zhang e B. Yang, "Box-cox transformation in big data", Technometrics, vol. 59, no. 2, pp. 189-201, 2017. 36

[59] G. E. P. Box e D. R. Cox, "An analysis of transformations revisited, rebutted", Journal of the American Statistical Association, vol. 77, no. 377, pp. 209-210, 1982. 38

[60] D. G. de Figueiredo, Análise de Fourier e equações diferenciais parciais. IMPA, 1977. 38

[61] N. Wiener, The Fourier Integral and certain of its applications. Cambridge University press, 1933. 40 
[62] R. A. Askey, M. Rahman e S. Suslov, "On a general q-fourier transformation with nonsymmetric kernels", Journal of Computational and Applied Mathematics, vol. 68 , no. 1 , pp. $25-55,1996.40$

[63] S. Umarov, C. Tsallis e S. Steinberg, "On a q-central limit theorem consistent with nonextensive statistical mechanics", Milan Journal of Mathematics, vol. 76, pp. 307-328, Dec 2008. 41, 56, 99

[64] H. J. Hilhorst, "Note on aq-modified central limit theorem", Journal of Statistical Mechanics: Theory and Experiment, vol. 2010, p. P10023, oct 2010. 41

[65] A. Plastino e M. Rocca, "Inversion of umarov-tsallis-steinberg's q-fourier transform and the complex-plane generalization", Physica A: Statistical Mechanics and its Applications, vol. 391, no. 20, pp. 4740 - 4747, 2012. 42, 45

[66] A. Plastino e M. Rocca, " $q$-fourier transform and its inversion-problem", Milan J. Math., vol. 80, no. 243, pp. 243-249, 2012. 42, 45

[67] A. Scarfone, "к-deformed fourier transform", Physica A: Statistical Mechanics and its Applications, vol. 480, pp. 63 - 78, 2017. 42, 72

[68] J. L. Walsh, "History of the riemann mapping theorem", The American Mathematical Monthly, vol. 80, no. 3, pp. 270-276, 1973. 42

[69] W. P. Calixto, "Aplicação do mapeamento conforme no cálculo do fator de carter", Dissertação de Mestrado, Universidade Federal de Goiás, 2008. 43

[70] S. G. Krantz, Handbook of complex variables. New York: Springer Science+Business Media, LLC, 1 ed., 1999. 44

[71] S. Umarov e C. Tsallis, "On a representation of the inverse fq-transform", Physics Letters A, vol. 372, no. 29, pp. 4874 - 4876, 2008. 45

[72] D. Sornette, "Discrete-scale invariance and complex dimensions", Physics Reports, vol. 297, no. 5, pp. 239 - 270, 1998. 46, 66

[73] D. Isbell, "Log periodic dipole arrays", IRE Transactions on Antennas and Propagation, vol. 8, pp. 260-267, May 1960. 59, 66 
[74] J. J. C. dos Santos, "Estudo e aplicações das funções hiperbólicas", Dissertação de Mestrado, Departamento de Matemática - Universidade Federal da Paraíba, 2015. 63

[75] S. Drożdż, F. Grümmer, F. Ruf e J. Speth, "Prediction oriented variant of financial log-periodicity and speculating about the stock market development until 2010", in Practical Fruits of Econophysics (H. Takayasu, ed.), (Tokyo), pp. 93-98, Springer Tokyo, 2006. 66

[76] V. Filimonov e D. Sornette, "A stable and robust calibration scheme of the log-periodic power law model", Physica A: Statistical Mechanics and its Applications, vol. 392 , no. 17 , pp. $3698-3707,2013.66$

[77] A. Pietak, S. Ma, C. W. Beck e M. D. Stringer, "Fundamental ratios and logarithmic periodicity in human limb bones", Journal of Anatomy, vol. 222, no. 5, pp. 526-537, 2013. 66

[78] K. Anim e Y. Jung, "Shortened log-periodic dipole antenna using printed dual-band dipole elements", IEEE Transactions on Antennas and Propagation, vol. 66, pp. 6762-6771, Dec 2018. 66

[79] A. Amini, H. Oraizi e M. A. Chaychi zadeh, "Miniaturized uwb log-periodic square fractal antenna", IEEE Antennas and Wireless Propagation Letters, vol. 14, pp. 1322-1325, 2015. 66

[80] J. C. Cressoni, G. M. Viswanathan e M. A. A. da Silva, "Log-periodicity in piecewise ballistic superdiffusion: Exact results", Phys. Rev. E, vol. 98, p. 052102, Nov 2018. 66, 99

[81] J. P. D'Incao, J. Wang e V. E. Colussi, "Efimov physics in quenched unitary bose gases", Phys. Rev. Lett., vol. 121, p. 023401, Jul 2018. 66

[82] A. M. Scarfone, "On the $\kappa$-deformed cyclic functions and the generalized fourier series in the framework of the $\kappa$-algebra", Entropy, vol. 17, no. 5, pp. 2812-2833, 2015. 72

[83] T. Kim e D. Kim, "Degenerate laplace transform and degenerate gamma function", Russian Journal of Mathematical Physics, vol. 24, pp. 241-248, Apr 2017. 
[84] V. Adamchik, "On stirling numbers and euler sums", Journal of Computational and Applied Mathematics, vol. 79, no. 1, pp. 119 - 130, 1997. 81

[85] W. Lang, "On generalizations of the stirling number triangles", Journal of Integer Sequences, vol. 3, no. 00.2.4, 2000. 81

[86] Y. A. Katz, "q-gaussian model of default: Valuation of cds spreads.", SSRN, p. $22,2018.99$

[87] L.-M. Liu, Y.-Y. Cui, J. Xu, C. Li e Q.-H. Gao, "The non-markovian property of q-gaussian process", Computers and Mathematics with Applications, vol. 79, no. 6, pp. $1802-1812,2020$.

[88] S. T. C. Hanel, R.; Thurner, "Limit distributions of scale-invariant probabilistic models of correlated random variables with the q-gaussian as an explicit example", The European Physical Journal B, vol. 72, p. 5, oct 2009.

[89] J.-F. Bercher, "On generalized Cramér-Rao inequalities, generalized Fisher information and characterizations of generalized q-Gaussian distributions", Journal of Physics A: Mathematical and Theoretical, vol. 45, p. 255303, may 2012. 99

[90] R. M. B. Diniz, J. C. Cressoni, M. A. A. da Silva, A. M. Mariz e J. M. de Araújo, "Narrow log-periodic modulations in non-markovian random walks", Phys. Rev. E, vol. 96, p. 062143, Dec 2017. 99 